Frederike C. A. Faupel

\title{
Die Betreuungsverfügung
}

Ein Instrument zur privatautonomen Ausgestaltung der gesetzlichen Betreuung

(D) weitmöglichste Menschen

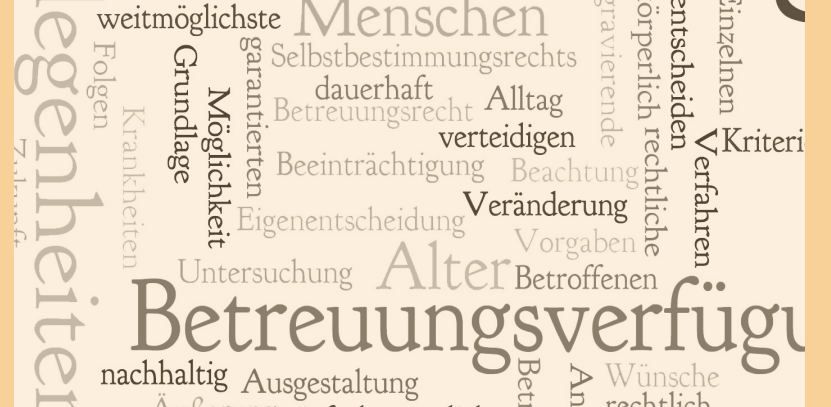

$\square$ Außerung einfachgesetzliche $\frac{O}{0}$ r rechtlich überraschend

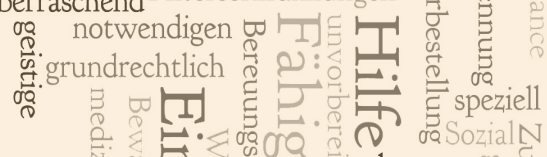

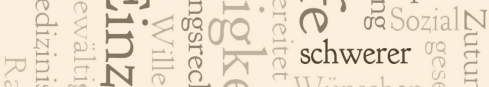

$$
\begin{aligned}
& \text { N } \sum_{i}^{2} Q \text {. Wünschen }{ }_{0}
\end{aligned}
$$

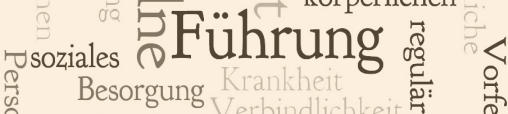



Frederike C. A. Faupel

Die Betreuungsverfügung

This work is licensed under the Creative Commons License 3.0 "by-nd", allowing you to download, distribute and print the document in a few copies for private or educational use, given that the document stays unchanged and the creator is mentioned.

You are not allowed to sell copies of the free version.

SORIERIGHIS RESERVED 
erschienen in der Reihe der Universitätsdrucke

im Universitätsverlag Göttingen 2010 
Frederike C. A. Faupel

Die Betreuungsverfügung

Ein Instrument zur

privatautonomen Ausgestaltung

der gesetzlichen Betreuung

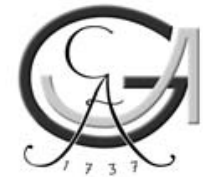

Universitätsverlag Göttingen 2010 


\section{Bibliographische Information der Deutschen Nationalbibliothek}

Die Deutsche Nationalbibliothek verzeichnet diese Publikation in der Deutschen Nationalbibliographie; detaillierte bibliographische Daten sind im Internet über $<$ http://dnb.ddb.de $>$ abrufbar.

Anschrift des Autors

Frederike C. A. Faupel

e-mail: frederike_faupel@web.de

Dieses Buch ist nach einer Schutzfrist auch als freie Onlineversion über die Homepage des Verlags sowie über den OPAC der Niedersächsischen Staats- und Universitätsbibliothek (http://www.sub.uni-goettingen.de) erreichbar und darf gelesen, heruntergeladen sowie als Privatkopie ausgedruckt werden. Es gelten die Lizenzbestimmungen der Onlineversion. Es ist nicht gestattet, Kopien oder gedruckte Fassungen der freien Onlineversion zu veräußern.

Satz und Layout: Frederike C. A. Faupel, Franziska Lorenz Umschlaggestaltung: Franziska Lorenz

(C) 2010 Universitätsverlag Göttingen http://univerlag.uni-goettingen.de ISBN: 978-3-941875-82-1 
Meinen Eltern 



\section{Vorwort}

Diese Arbeit wurde im Dezember 2009 fertig gestellt und im Wintersemester 2009/2010 von der Juristischen Fakultät der Georg-August-Universität Göttingen als Dissertation angenommen. Sie entstand im Rahmen des von der Deutschen Forschungsgemeinschaft (DFG) geförderten Projektes „Perspektiven der Selbstbestimmung in der alternden Gesellschaft" (Geschäftszeichen: LI 892/2-1). Danken möchte ich der Dr.-Carl-Böse-Stiftung zu Lübeck für die Übernahme der Druckkosten.

Mein herzlicher Dank gilt meinem Doktorvater, Herrn Prof. Dr. Volker Lipp, der diese Arbeit angeregt hat. Während ihrer Erstellung wusste er in zahlreichen Diskussionen neue Impulse zu setzen. Für anregende Gespräche nahm er sich stets Zeit und erbrachte - im besten Sinne des Wortes - eine exzellente Betreuung. Auch darüber hinaus förderte er mich während meiner Tätigkeit an seinem Lehrstuhl stets großzügig und stand meiner Beteiligung an weiterführenden wissenschaftlichen Tätigkeiten und Diskussionen stets offen und unterstützend gegenüber.

Auch danke ich Frau Prof. Dr. Barbara Veit für die Anfertigung des Zweitgutachtens.

Weiter danke ich Monika Schiller, geb. Burchardt für die geführten Diskussionen und die gemeinsame Arbeit im Rahmen des DFG Forschungsvorhabens „Perspektiven der Selbstbestimmung in der alternden Gesellschaft". Für die Unterstützung und stets aufmunternden Worte danke und ich darüber hinaus dem ehemaligem Lehrstuhl- 
kolleginnen und -kollegen, den Sekretärinnen Frau Marie-France Hesse und Frau Susanne Kirchboff und den Kollegen Schirin Rüger und Andreas Lönner sowie Annemarie Faupel für die Korrektur der Arbeit.

Die Arbeit widme ich meinen Eltern, deren unerschütterliches Vertrauen und bedingungslose Liebe mich nicht nur durch das Studium und die Promotion begleitet haben.

Zuletzt möchte ich meinen herzlichen Dank meinem langjährigen Freund und heutigen Ehemann Florian aussprechen. Er hat mir Studium und Promotion nahe gelegt und sich mit mir über deren Sinn und Inhalte über die Jahre in zahllosen Diskussionen auseinandergesetzt und mich dadurch fortwährend liebevoll und hilfreich unterstützt.

Lübeck, im Oktober 2010

Frederike C.A. Faupel, geb. Klein 


\section{Inhaltsverzeichnis}

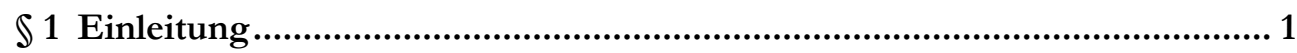

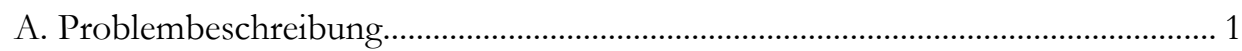

I. Abgrenzung zur Vorsorgevollmacht und zur Patientenverfügung ...................... 3

II. Möglichkeiten der Betreuungsverfügung............................................................. 5

B. Weiterer Gang der Untersuchung ....................................................................... 7

\section{$\$ 2$ Betreuungsverfügung als Instrument}

zur Ausübung des Selbstbestimmungsrechts .............................................. 9

A. Verfassungsrechtliche Grundlagen des Selbstbestimmungsrechts ........................ 9

B. Ausübung des Selbstbestimmungsrechts

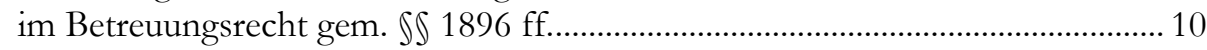

I. Betreuung als Fremd- bzw. Selbstbestimmung ................................................... 10

II. Aufgaben und Ziele der Betreuung .................................................................. 11

1. Rechtliche Stellung des Betreuten ....................................................... 12

2. Aufgaben, Handlungs- und Entscheidungsmaßstab

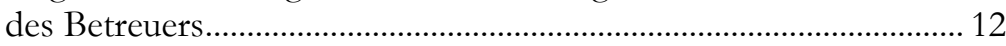


3. Private Vorsorge als Ziel der Gesetzgebung........................................ 13

III. Selbstbestimmungsrecht in der Betreuung ..................................................... 15

C. Antizipierte Ausübung des Selbstbestimmungsrechts mittels der Betreuungsverfügung ................................................................... 15

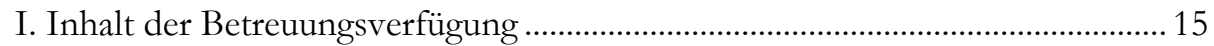

II. Wirkung der Betreuungsverfügung.................................................................. 17

1. Wunsch des Betroffenen.................................................................. 17

a) Einwilligungs- und Geschäftsfähigkeit des Betroffenen ..........18

b) Auslegung der Betreuungsverfügung ......................................... 20

c) Wirkung der Betroffenenwünsche ……………………………...... 22

aa) Innenwirkung ................................................................ 22

bb) Außenwirkung ……………………………………...... 22

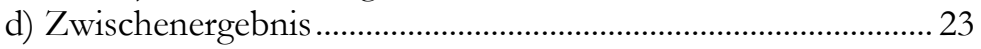

2. Geltung antizipiert geäußerter Wünsche in der Betreuung ............... 23

a) Vorrang des aktuellen Wunsches ................................................ 23

b) Beachtlichkeit antizipiert geäußerter Wünsche........................... 24

c) Voraussetzung für die Bindung.................................................... 26

d) Fehlender Widerruf..................................................................... 27

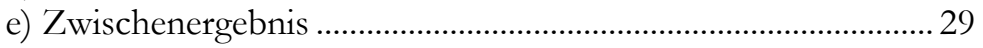

3. Entfallen der Bindung an den Betroffenenwunsch ........................... 29

a) Fehlender freier und eigenverantwortlicher Wille ...................... 30

b) Drohende Selbstschädigung ........................................................ 30

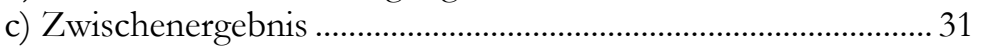

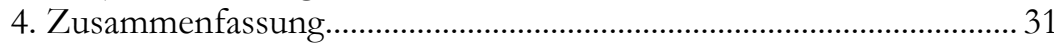

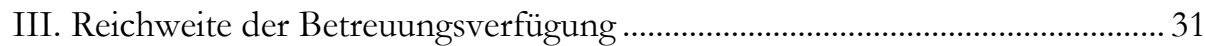

1. Wohl des Betroffenen gem. \ 1901 Abs. 3 S. 1 ................................... 32

a) Problemerörterung ……………………………………............ 32

b) Verständnis von „Wohl“ in der Betreuung................................. 34

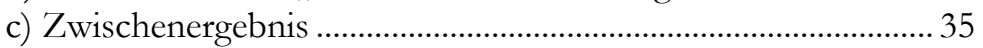

2. Zumutbarkeit für den Betreuer gem. \1901 Abs. 3 S. 1 ................... 36

3. Gesetzlich zwingende Regelungen ....................................................... 37

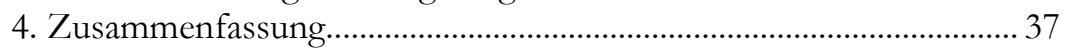

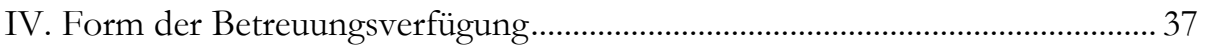

V. Sicherstellen der Wirkung der Betreuungsverfügung............................................ 39

1. Registrierung im Zentralen Vorsorgeregister........................................ 39

2. Ablieferung der Betreuungsverfügung bei Gericht gem. \ 1901c ....................................................................... 41

3. Beratung beim Abfassen der Betreuungsverfügung .......................... 44

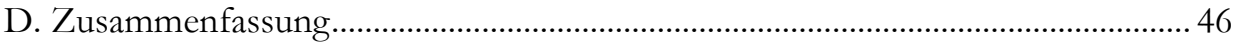




\section{\3 Einfluss der Betreuungsverfügung} auf die Auswahl des Betreuers ..................................................................... 49

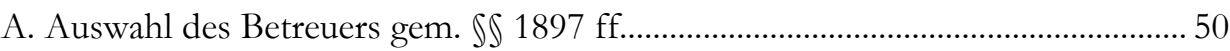

I. Vorschlag gem. \1897 Abs. 4 S. 1 ................................................................. 50

1. Einzelperson, Personenkreis oder -gruppe ……………....................... 50

2. Gegenbetreuer ………………………………………….................... 53

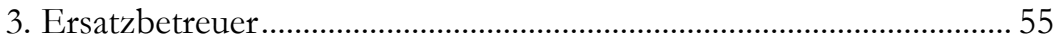

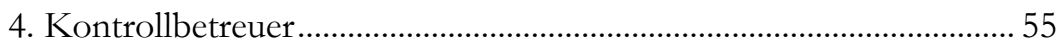

II. Vorschlag gem. \ 1897 Abs. 4 S. 2 2................................................................. 56

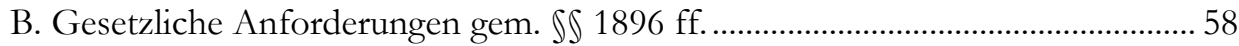

I. Eignung der vorgeschlagenen Person gem. \1897 Abs. 1 ................................... 59

II. Vorrang der privaten, auch berufsmäßigen Einzelbetreuung

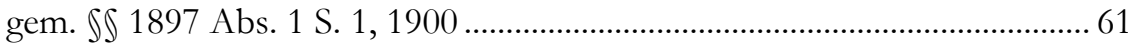

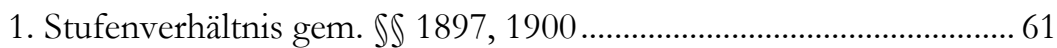

2. Auswahl von Vereinsmitglieder gem. \1900 Abs. 2 S. 2................... 63

III. Ausschluss der in $\ 1897$ Abs. 3 genannten Personen ........................................ 63

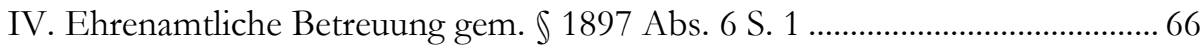

V. Übernahmepflicht und Bereiterklärung gem. \ 1898 ............................................68

VI. Geschäftsfähigkeit und Volljährigkeit des Betreuers........................................ 71

VII. Keine eigene Betreuungsbedürftigkeit des Betreuers ....................................... 72

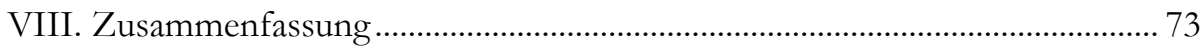

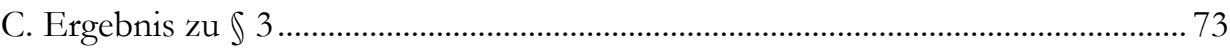

\section{$\$ 4$ Einfluss der Betreuungsverfügung} auf das Verfahren der Betreuerbestellung................................................... 75

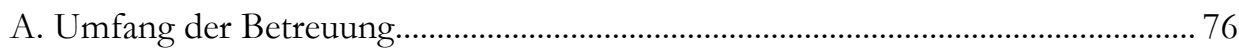

I. Bestimmung der Aufgabenkreise gem. \1901 Abs. 1.......................................... 76

II. Typisierung der Aufgabenkreise nach Rechtsprechung und Literatur............. 77

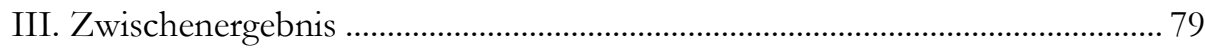

B. Verfahrenspfleger gem. \ 276 FamFG ............................................................... 82

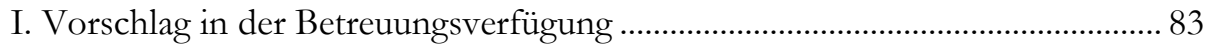

1. Auswahl einer Person .......................................................................... 83

2. Ausschluss einer Person ........................................................................... 85 
II. Vermeidung der Verfahrenspflegschaft durch privatautonome

Bevollmächtigung eines Dritten oder Verzicht auf den

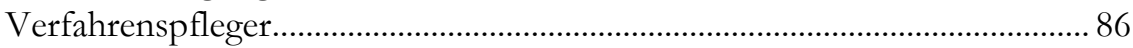

1. Bevollmächtigte Verfahrensvertreter .................................................... 86

2. Verzicht auf den Verfahrenspfleger ................................................. 87

a) Bestellung eines Verfahrenspflegers trotz

Verfahrensbevollmächtigten ................................................. 87

b) Bestellung eines Verfahrenspflegers gegen den

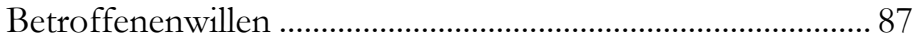

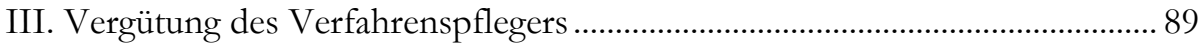

C. Anhörung des Betroffenen gem. \278 FamFG ..................................................... 92

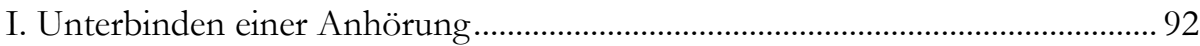

II. Anhörungsort gem. \278 Abs. 1 FamFG ............................................................. 94

III. Hinzuziehung von Vertrauenspersonen und Ausschluss

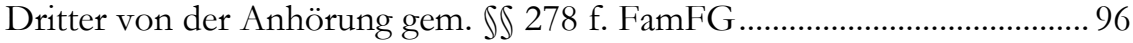

1. Hinzuziehung von Vertrauenspersonen ........................................... 96

2. Ausschluss Dritter ................................................................................ 99

IV. Anhörung von Vertrauenspersonen und Ausschluss der Anhörung Dritter gem. $\iint 278,274$ FamFG .......................................... 100

D. Sachverständigengutachten gem. $\iint 280$ ff. FamFG ............................................ 102

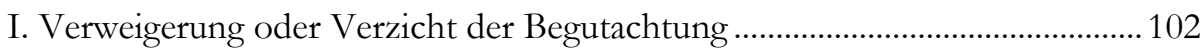

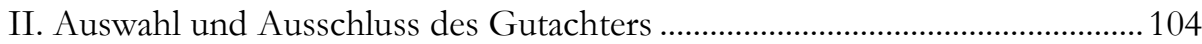

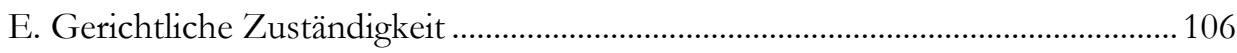

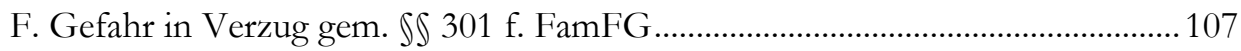

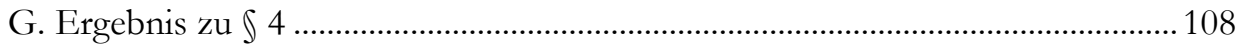

\section{\5 Einfluss der Betreuungsverfügung auf die Betreuung}

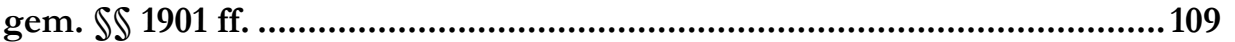

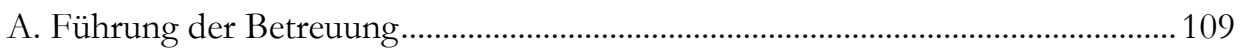

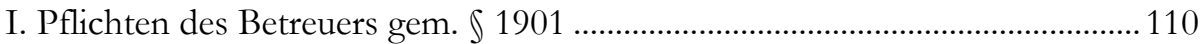

1. Aufgabenkreis und persönliche Betreuung .................................... 110

2. Wünsche und Vorstellungen des Betreuten ..................................... 111

II. Medizinische Behandlung gem. Sf 1901a, 1901b ............................................ 112 
III. Genehmigungsvorbehalte der $\iint 1904$ - 1908, 1908i ................................... 113

1. Sterilisation des Betreuten gem. \ 1905 ............................................. 115

2. Unterbringung des Betreuten gem. \ 1906...................................... 116

3. Übrige Angelegenheiten des Betreuten........................................... 118

a) Fragen der Gesundheit ......................................................... 118

b) Fragen des Aufenthalts und des Wohnraums........................ 120

c) Vermögenssorge ...................................................................... 122

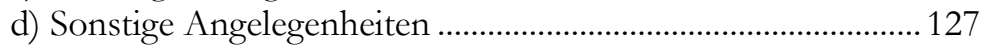

4. Zwischenergebnis ............................................................................ 127

IV. Stellung, Aufsicht und Pflichten des Betreuers ............................................. 128

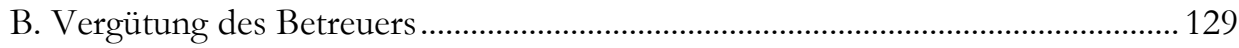

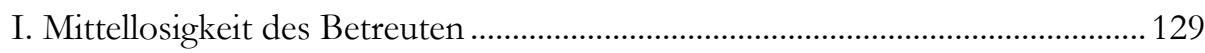

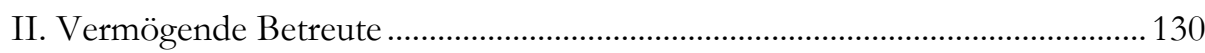

1. Individuelle Vergütung des Berufsbetreuers ................................... 130

a) Vormünder- und Betreuervergütungsgesetz .......................... 130

b) Alternative Vereinbarungen.................................................... 132

2. Individuelle Vergütung des ehrenamtlich tätigen Betreuers........... 133

a) Gestaltungsmöglichkeiten durch die

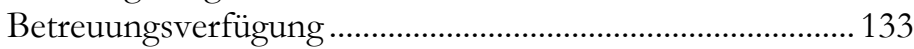

b) Individuelle Bestimmung der Vergütungshöhe...................... 134

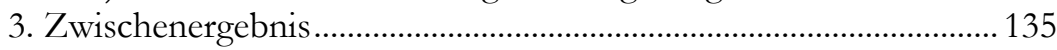

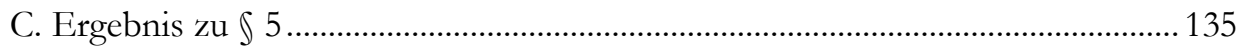

S 6 Missachtung der Betreuungsverfügung ...........................................137

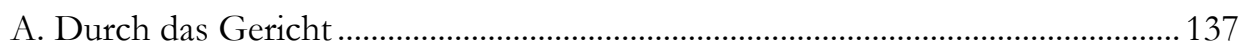

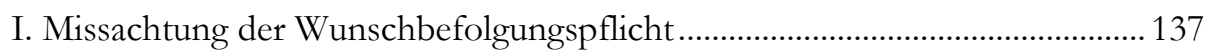

II. Rechtsfolgen und Beschwerdeberechtigung .................................................. 138

1. Einrichtung der Betreuung von Amts wegen .................................. 139

a) Gesetzesbegründung ................................................................ 140

b) Beschwerderecht des abgelösten Betreuers............................ 141

c) Rechtsinstitut Vorsorgevollmacht............................................... 142

d) $\iint 1776,1779$ und Art. 6 Abs. 1 GG ...................................... 144

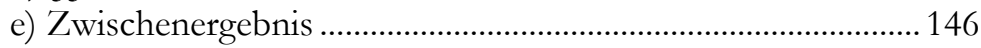

2. Einrichtung der Betreuung auf Antrag des Betroffenen................. 147 
B. Durch den Betreuer ............................................................................................ 148

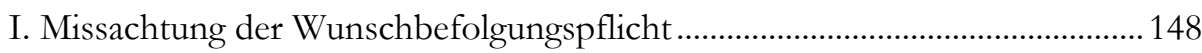

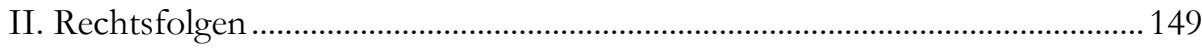

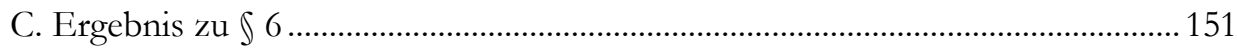

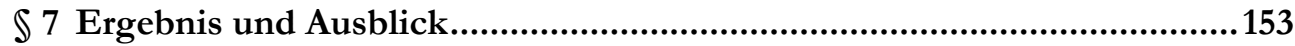

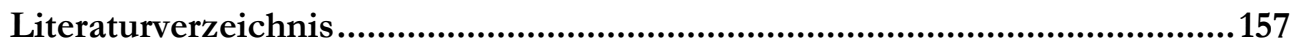

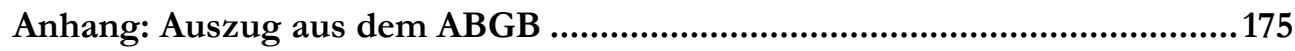

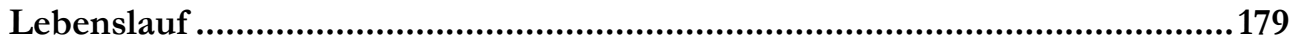




\section{Abkürzungen}

Es werden die üblichen Abkürzungen nach Hildebert Kirchner, Abkürzungsverzeichnis der Rechtssprache, 6. Auflage, Berlin 2008 und Wermke, Matthias/ Kunkel-Razum, Kathrin/Scholze-Stubenrecht, Werner, DUDEN Band 1, Die deutsche Rechtschreibung, 25. Auflage, Mannheim, Leipzig, Wien, Zürich 2009 verwendet. 



\section{$\$ 1$ Einleitung}

\section{A. Problembeschreibung}

Ein schwerer Unfall und seine gesundheitlichen Folgen oder das Auftreten einer gravierenden Krankheit verändern das Leben eines Menschen zumeist unerwartet und nachhaltig. Mit dem Alter nehmen körperliche Gebrechlichkeit sowie alterstypische Krankheiten, wie z.B. Demenz, zu und beeinträchtigen das Leben dauerhaft. Den Betroffenen und sein soziales Umfeld trifft dies häufig überraschend und unvorbereitet. Sein Leben ändert sich gegen seinen Willen und ohne sein Zutun. Er bedarf fortan fremder Hilfe zur Bewältigung seines Alltags.

Besonders ältere Menschen sind in der Besorgung ihrer Angelegenheiten eingeschränkt. Die reguläre, altersbedingte medizinische Degeneration von geistigen und körperlichen Fähigkeiten, aber auch spezielle Alterserkrankungen begründen ihre Hilfsbedürftigkeit. Nicht nur die Besorgung ihrer Angelegenheiten, sondern auch die Fähigkeit, selbst zu entscheiden, wie das eigene Leben ausgestaltet sein soll, nimmt ab. Zunehmend fällt es schwerer, eigene Entscheidungen zu treffen, sie umzusetzen und anderen gegenüber zu verteidigen. Durch den demographischen Wandel steigt die Zahl der Betroffenen stetig an. Der Anteil der über 65jährigen lag in der Bevölkerung der Bundesrepublik Deutschland im Jahr 2005 
bei knapp 20 Prozent, im Jahr 2050 wird er voraussichtlich bei über 33 Prozent liegen. ${ }^{1}$

Die Situation, in der der Einzelne nicht mehr fähig ist, eigene Entscheidungen zu treffen bzw. getroffene Entscheidungen umzusetzen, bereitet rechtliche Schwierigkeiten. Fraglich ist, wer die erforderliche Hilfe leistet und die notwendigen rechtlichen Angelegenheiten an Stelle des Betroffenen übernimmt. Das Gesetz sieht hierfür in den $\$ \int 1896$ ff. BGB2 die Betreuung vor. Der rechtliche Betreuer $^{3}$ erhält im erforderlichen Aufgabenbereich im Außenverhältnis kraft Gesetz Stellvertretungsbefugnis, um die Angelegenheiten des Betroffenen rechtswirksam umzusetzen, vgl. \1896 Abs. 1 und 2, \$ 1902. Diese hat der Betreuer so zu erledigen, wie es den Wünschen des Betreuten entspricht, vgl. \1901 Abs. 2 und 3. Ein gutes Verhältnis zwischen beiden ist als Kommunikationsgrundlage hilfreich, aber nicht Voraussetzung. Ist der Betreuer eine Person des Vertrauens des Betreuten, erleichtert dies die Situation. Kennt der Betreuer den Betroffenen aus „seinem früheren Leben“, kennt er auch dessen Lebensgewohnheiten und seinen Alltag. \1897 Abs. 4 und \1901 Abs. 2 sehen vor, dass der Betroffene Vorschläge zur Auswahl seines Betreuers und Wünsche zur Einrichtung und Führung seiner Betreuung abgeben kann. Ist er dazu nicht mehr in der Lage und können seine Wünsche auch nicht über Dritte erfragt oder sonst herausgefunden werden, können sie nicht berücksichtigt werden. Ihr Fehlen zwingt das Betreuungsgericht ${ }^{4}$ und den Betreuer auf einen objektiven Maßstab zurück zu greifen und im besten Sinn „für" den Betroffenen zu entscheiden. Bleiben nur Sekundärinformation durch Angehörige und Bekannte des Betroffenen, könnten deren Aussagen aufgrund anderweitiger Interessen nicht ausschließlich auf das Wohl des Betroffenen, sondern beispielsweise auf das eigene (Erb-) Interesse gerichtet sein.

Diese Gefahr der Fremdbestimmung kann der Betroffene abwenden, indem er vorab im Rahmen einer Betreuungsverfügung seinen Wunschbetreuer bestimmt und Wünsche betreffend der Einrichtung sowie der Führung der Betreuung festhält, die das Gericht und der Betreuer dann ihren Entscheidungen zugrunde legen können. Die Bedeutung der antizipierten Äußerung von derartigen Wünschen in

1 Statistisches Bundesamt, S. 57.

2 Alle folgenden Paragrafen ohne Gesetzesangabe sind solche des BGB.

${ }^{3}$ Zur besseren Lesbarkeit wird im Folgenden für die Personenbezeichnung das generische Maskulinum gewählt, gleichwohl sind männliche und weibliche Personen gleichermaßen gemeint.

${ }^{4}$ Durch das Gesetz über das Verfahren in Familiensachen und in den Angelegenheiten der freiwilligen Gerichtsbarkeit (FamFG) wurde die sachliche Zuständigkeit für Betreuungssachen gem. \23a Abs. 1 Nr. 2, Abs. 2 Nr. 1 GVG zum 01.09.2009 geändert. Unverändert ist die Zuständigkeit der Amtsgerichte. Bei diesen werden gem. $\ 23$ c Abs. 1 GVG Abteilungen für Betreuungssachen, Unterbringungssachen und betreuungsgerichtliche Zuweisungssachen gebildet, sog. Betreuungsgerichte. Die vorherige Bezeichnung Vormundschaftsgericht wird nicht mehr verwandt. In der vorliegenden Arbeit wird entsprechend der aktuellen gesetzlichen Regelung die Bezeichnung Betreuungsgericht genutzt. Bei Verweisen aus früherer Zeit, werden die Gerichtsbezeichnungen synonym verwandt und daher grundsätzlich entsprechend aktuell angeglichen. Sollte dies nicht möglich sein, wird ausdrücklich darauf hingewiesen. 
Form der Betreuungsverfügung wird mit Blick auf die stetig zunehmende Zahl zu betreuender Menschen deutlich. ${ }^{5}$ Eine individuelle und zeitintensive Betreuung, die am Wunsch und am Interesse des Betreuten ausgerichtet ist und diese gewissenhaft ermittelt, scheint bedroht. Waren es im Jahr 1995 noch knapp 625.000, ${ }^{6}$ gab es im Jahr 2005 bereits mehr als 1.2 Mio. Betreute: ${ }^{7}$ Dies ist ein Anstieg von fast $100 \%$ in nur zehn Jahren. Dabei dominieren demenzielle Erkrankungen mit $46 \%$ die Gründe der Betreuerbestellung. ${ }^{8} 32 \%$ der eingerichteten Betreuungen betreffen über 70jährige. ${ }^{9}$ Mit dem demographischen Wandel steigt auch das Bedürfnis nach adäquaten rechtlichen Regelungsmöglichkeiten der auftretenden tatsächlichen Probleme. Dabei ist die grundlegende Frage, wer die Entscheidungen für und an Stelle des Betreuten über dessen Angelegenheiten des alltäglichen Lebens trifft. Ist er dazu tatsächlich nicht mehr in der Lage, hat er die Entscheidungen aber bereits antizipiert im Rahmen der Betreuungsverfügung getroffen, müsste auf diese zurückgegriffen werden können. Deren Verbindlichkeit und mögliche Inhalte sowie die Voraussetzungen des Rechtsinstituts Betreuungsverfügung, werden in der vorliegenden Arbeit untersucht.

\section{Abgrenzung zur Vorsorgevollmacht und zur Patientenverfügung}

Kann eine Person ihre Rechtsangelegenheiten nicht mehr erledigen, bedarf sie der Hilfe Dritter. Unsere Rechtsordnung sieht für diesen Fall mehrere Lösungen vor. Zum einen erklärt sie den Betroffenen nach $\iint$ 104, 105 für einwilligungs- und geschäftsunfähig. ${ }^{10}$ Zum anderen eröffnet sie mittels so genannter Vorsorgeinstrumente dem Einzelnen die Möglichkeit einer antizipierten Regelung seiner Rechtsangelegenheiten und stellt ihm darüber hinaus die rechtliche Betreuung nach SS 1896 ff. zur Seite. Zwischen den privatrechtlichen Vorsorgeinstrumenten - der Vorsorgevollmacht und der Patientenverfügung - und der gesetzlich umfassend geregelten und staatlich kontrollierten rechtlichen Betreuung ist $\mathrm{zu}$ unterscheiden.

Eine rechtliche Betreuung wird durch das Betreuungsgericht eingerichtet. Dieses kontrolliert als Organ der Rechtspflege die Notwendigkeit und Führung der Betreuung und hebt sie ggf. auf, vgl. \ 1896 Abs. 1, 2, \\ 1908d, 1908i i.V.m. $\int 1837$ Abs. $1-3$.

Die Vorsorgevollmacht stellt eine echte Alternative zur Betreuung dar, vgl. ऽ 1896 Abs. 2 S. 2. Mit ihr erteilt der Vollmachtgeber dem Vollmachtnehmer (auch) für die Zeit künftiger eigener Hilflosigkeit die rechtliche Stellung, alle in der

\footnotetext{
5 Zum Einfluss der Demographie auf die Betreuungszahlen: Korte FPR 2004, 643, 646.

${ }^{6}$ Sellin/Engels/Holzhauer S. 21.

7 Deinert BtPrax 2007, 3; vgl. auch Köller/Sellin/Engels S. 32 ff.

8 Sellin/Engels/Holzhauer S. 54.

9 Sellin/Engels/Holzhamer S. 53.

10 Unter Beachtung der Ausnahmeregelung \105a, wonach ein volljähriger Geschäftsunfähiger Geschäfte des täglichen Lebens wirksam abschließen kann.
} 
Vollmacht genannten Angelegenheiten des Vollmachtgebers für ihn und an dessen Stelle zu erledigen. ${ }^{11}$ Der Vollmachtnehmer erhält in den benannten Angelegenheiten Vertretungsbefugnis nach den allgemeinen Regeln des Vertretungsrechts. ${ }^{12}$

Rechtswissenschaftlich ist die Vorsorgevollmacht ausführlich untersucht, ${ }^{13}$ tatsächlich aber trotz vielfältiger Fördermaßnahmen relativ wenig verbreitet ${ }^{14}$. Dies dürfte darin begründet sein, dass die Vorsorgevollmacht angesichts der restriktiven Praxis zum Rechtsberatungsgesetz (RBerG) ${ }^{15}$ nur dem Ehegatten, dem Lebenspartner, einem engen Verwandten oder einem Rechtsanwalt erteilt werden durfte. ${ }^{16}$ Darüber hinaus setzt die Vollmachtserteilung wegen der Gefahr des Missbrauchs ${ }^{17}$ ein hohes Maß an Vertrauen in den Vollmachtnehmer voraus. Dieser muss sich nur dem Vollmachtgeber gegenüber rechtfertigen. Zu dieser Kontrolle ist der Vollmachtgeber in den Situationen, für die er eine Vollmacht erteilt, jedoch häufig gerade nicht mehr in der Lage. Eine der Betreuung vergleichbaren Kontrolle durch das Betreuungsgericht ist im Rahmen der Vorsorgevollmacht grundsätzlich nicht gegeben. ${ }^{18}$

Mit Hilfe einer Patientenverfügung möchte der künftige Patient vorsorglich das „Ob“ und „Wie“ einer späteren medizinischen Behandlung festlegen. Ihre Reichweite und ihre rechtliche Bindungswirkung waren in Rechtsprechung und Wissenschaft, aber auch rechtspolitisch sehr umstritten. ${ }^{19}$ Ihre Verbindlichkeit wurde durch das 3. Gesetz zur Änderung des Betreuungsrechts (3. BtÄndG) nun in den $\iint 1901 \mathrm{a}$ Abs. 1, 1901b festgelegt. ${ }^{20}$ Beim Abfassen einer Patientenverfügung wird meist übersehen, dass sie nur einige wenige, wenngleich wichtige ärztliche Maßnahmen vorsorglich regeln kann, alle anderen nicht medizinischen Fragen

11 Walter S. 2.

12 Zimmermann Rn. 26 ff.

${ }^{13}$ Aktueller Überblick bei Staudinger/Bienwald \$ 1896 Rn. 114 ff.; weitergehend für die Vollmacht: Bawer S. 27 ff.; Langenfeld S. 5 ff. und Walter S. 43 ff.; i.Ü. MünchKommBGB/Schwab \ 1896 Rn. 47 ff.; Abrens BtPrax 2005, 163 ff.; Bienwald BtPrax 1998, 164 ff. und 2002, 227 ff.; Binschus Der Amtsvormund 1998, 275 ff.; Bübler BWNotZ 1990, 1 ff.; Coeppicus Sterbehilfe S. 111 ff.; Milzer NJW 2003, 1836 ff.; Perau MittRhNotK 1996, 285 ff.; Rieger FS Schwab S. 1043 ff.; Schwab K 27 ff.; Ublenbruck Selbstbestimmtes Sterben S. 326 ff.; Zimmermann S. 41 ff.

${ }^{14}$ Köller/Sellin/Engels S. 42 ff.; Simon/Meran/Fangerau Der Hautarzt 2004, 721, 724. V.a. die Akzeptanz bei Banken und Sparkassen ist nicht gewährleistet (BT-Drucks. 15/2494 S. 15).

${ }^{15}$ Das Rechtsberatungsgesetz (RBerG) wurde mit Wirkung zum 01.07.2008 durch das Gesetz über außergerichtliche Rechtsdienstleistung (RDG) ersetzt. Ob die restriktive Praxis zur Vollmachtserteilung auch nach dem RDG nur gegenüber diesem Personenkreis möglich ist, ist fraglich.

16 Noch zum RBerG: Ahrens BtPrax 2005, 163, 166; Lipp FS Bienwald S. 177.

${ }^{17}$ Vgl. Bienwald BtPrax 1998, 164 ff. und BtPrax 1999, 92 ff.; Bawer Versorgung und Vorsorge S. 27 ff.; Walter S. $160 \mathrm{ff}$.

18 Anders ist dies im Fall des Kontrollbetreuers nach $\$ 1896$ Abs. 3, vgl. dazu $\ 3$ A. I. 4.

${ }^{19}$ BGHSt 40, 257 ff.; BGHZ 154, 205 ff.; BGHZ 163, 195 ff.; Taupitz. A 36 ff.; Verrel C 46 ff.; ausführlich Lipp Patientenautonomie S. 21 ff.; Anhörung im Rechtsausschuss des Bundestag am 04.03.2009 zur Debatte über die Gesetzesentwürfe zur Regelung von Patientenverfügungen, vgl. BT-Drucks. 16/13314; Der Spiegel 2008 Nr. 48 S. 164 ff.

20 Zum 3. BtÄndG und der neuen Regelungen zur Patientenverfügung vgl. \5 A. II. und Fn. 78, 684. 
aber unbeantwortet bleiben, wie etwa die finanziellen Angelegenheiten oder der Umzug in ein Pflegeheim. Sie macht daher die Bestellung eines Betreuers i.d.R. nicht entbehrlich. ${ }^{21}$ Im Übrigen deuten Studien darauf hin, dass die Patientenverfügung von der Bevölkerung wenig genutzt wird. ${ }^{22}$

\section{Möglichkeiten der Betreuungsverfügung}

Die Betreuungsverfügung ist in der Diskussion um die privatautonome Vorsorge bislang weitgehend außer Acht geblieben. Mittels der Betreuungsverfügung kann jeder Einzelne zum einen dem Betreuungsgericht eine Person als Betreuer vorschlagen, vgl. IS 1897 Abs. 1, 1901c, zum anderen Wünsche für die Führung der Betreuung äußern, die der spätere Betreuer zu beachten hat, vgl. \$S 1901 Abs. 3 S. 2, 1901c. Die Betreuungsverfügung eröffnet dem Einzelnen somit die Möglichkeit, die gesetzliche Betreuung privatautonom auszugestalten und stellt eine Möglichkeit zur antizipierten Ausübung des Selbstbestimmungsrechts dar.

Die Betreuungsverfügung unterliegt nicht den genannten Beschränkungen der Vorsorgevollmacht. Vielmehr kann in ihr jede Person als Betreuer vorschlagen werden, \1897 Abs. 1 und 4. ${ }^{23}$ Auch sind die Missbrauchsmöglichkeiten geringer, weil der rechtliche Betreuer durch das Betreuungsgericht kontrolliert wird bzw. diesem regelmäßig Rechenschaft ablegen muss, vgl. SS 1904 Abs. 1, 1906 Abs. 1, 1907 Abs. 1, 1908 i Abs. 1. Sie verschafft daher all denjenigen die Möglichkeit zur vorsorglichen Ausübung ihrer Rechte, die keine Person ihres Vertrauens haben oder dieser aus Rechtsgründen keine Vollmacht erteilen können. ${ }^{24}$

Somit wird das Rechtsinstitut Betreuungsverfügung durch eine Mischung aus Privatautonomie und staatlicher Kontrolle charakterisiert. Vereint sie die jeweiligen Vorteile, könnte sie eine einzigartige Möglichkeit zur antizipierten Ausübung des Selbstbestimmungsrechts sein.

Seit Inkrafttreten des Betreuungsrechts im Jahr 1989 fanden zahlreiche Gerichtsverfahren statt, in denen sich die Gerichte mit einschlägigen Fragen des Betreuungsrechts auseinander setzen mussten. Viele dieser Verfahren beschäftigten sich mit dem Willen des Betreuten und den gesetzlichen Grenzen seiner Umsetzbarkeit. ${ }^{25}$ Nur in wenigen Fällen war der in Form einer Betreuungsverfügung antizipiert geäußerte Wille Gegenstand der Entscheidung. Selbst wo dies der Fall

\footnotetext{
${ }^{21}$ Lipp Patientenautonomie S. 32 f. In vielen Fällen wird trotz einer Patientenverfügung ein Betreuer auch für Gesundheitsangelegenheiten bestellt, was das BVerfG für verfassungsgemäß erachtete (BVerfG NJW 2002, 206).

22 Deutsche Hospizstiftung S. 3; van Oorschot, Ethik in der Medizin 2004, 112 ff.

${ }^{23}$ Eignung wird hier als faktischer Begriff verstanden (vgl. die Ausführungen zu 3. A. I.).

${ }^{24}$ Dodegge/Roth C. Rn. 80 ff.

25 Vgl. nur OLG Schleswig BtPrax 2008, 36; LG Zweibrücken BtPrax 2006, 154 f.; OLG Köln OLGR 2002, $337 \mathrm{f}$.
} 
war, wurde der einschlägige Sachverhalt in der (Fach-) Öffentlichkeit überwiegend nicht als Frage der Betreuungsverfügung wahrgenommen. ${ }^{26}$

Rechtswissenschaftlich wird die Betreuungsverfügung v.a. als Mittel zur Absicherung einer Vorsorgevollmacht diskutiert. ${ }^{27}$ Als eigenständiges Vorsorgeinstrument ist sie nur ansatzweise, ${ }^{28}$ ihre Grenzen und Möglichkeiten rechtswissenschaftlich so gut wie nicht erforscht. Eine umfassende Auseinandersetzung liegt nicht vor. ${ }^{29}$ Dieser Aufgabe stellt sich die vorliegende Arbeit. Hier sollen die wesentlichen, in Verbindung mit dem Rechtsinstitut der Betreuungsverfügung stehenden juristischen Fragen aufgeworfen, ihre Problematik erörtert und Antworten gefunden werden.

In rechtstatsächlicher Hinsicht hat die eigenständige Betreuungsverfügung nach ersten durchgeführten stichprobenartigen Untersuchungen ${ }^{30}$ vermutlich so gut wie keine Verbreitung gefunden. ${ }^{31}$ Die Gründe dafür sind in der fehlenden rechtswissenschaftlichen Untersuchung und der sich darin begründenden fehlenden Kenntnis über die Möglichkeiten und die Reichweite des Instruments zu vermuten. Dies bewirkt eine fehlende bzw. falsche Aufklärung über ihre Anwendungsmöglichkeiten. Dem widerspricht, dass bei 78,57 \% der Eintragungsanträge $^{32}$ im Zentralen Vorsorgeregister ${ }^{33}$ des Jahres 2008 angegeben wurde, dass auch eine Betreuungsverfügung bestehe. Aufgrund der sich abzeichnenden Unkenntnis in der Bevölkerung und in Fachkreisen ist zu vermuten, dass mit diesen Angaben zumeist nicht die Betreuungsverfügung im Sinne des Gesetzes oder die Betreuungsverfügung in ihrer rudimentären Grundstruktur gemeint ist. Unter letzterem ist die Betreuungsverfügung im Rahmen sog. Vorsorgepakete zu verstehen, ${ }^{34}$ die

26 Vgl. nur BGHZ 154, 205 ff. - Die Entscheidung wurde jedoch als Frage der Verbindlichkeit der Patientenverfügung wahrgenommen (Ublenbruck. NJW 2003, 1710 ff.; Deutsch NJW 2003, 1567 ff.; Lipp BtPrax 2004, 18 ff.).

27 V.a. wird sie in sog. Vorsorgepaketen genannt, bezieht sich dann aber zumeist ausschließlich auf die Benennung des Wunschbetreuers, vgl. nur Verbraucherzentrale NRW e.V.S. 50 ff.; Klinger/Klinger S. 100 ff.; Jülicher/Klinger S. 32 ff.; weitergehend bereits: Müller/Renner Rn. 318 ff. und Bittler S. 75 ff.; Geckle S. 76 f.; Birmanns Muster in NWB Nr. 52/53 vom 21.12.1998 S. 4355 f.; Winkler S. $55 \mathrm{ff}$.

28 Überblick bei Lipp FS Bienwald S. 177 ff. und Vorsorgeverfügungen $\ 18$; vgl. i.Ü. Bienwald BtPrax 1998, 164 ff.; Epple BWNotZ 1992, 27 ff. und BtPrax 1993, 156 ff.; Langenfeld S. 156 ff.; Peran MittRhNotK 1996, 285 ff.; Ublenbruck Patiententestament S. 16 ff. und Selbstbestimmtes Sterben S. 320 ff; Zimmermann S. 30, 209 ff.; Binschus ZfF 2000, 80 ff.; v. Looz BtPrax 2002, 179 ff.

${ }^{29}$ Siehe jedoch: Lipp FS Bienwald S. 177 ff.; Epple BWNotZ 1992, 27 ff. und BtPrax 1993, 156 ff.; Perau MittRhNotK 1996, 285 ff.

${ }^{30}$ Erhebungen der Stadt Göttingen, Fachbereich Soziales aus dem Jahr 2004 (unveröffentlicht).

${ }^{31} \mathrm{Zu}$ dieser Feststellung kommt auch Rieger FS Schwab S. 1044.

32 Die Zahl der Eintragungsanträge bezieht sich dabei lt. des Jahresberichts der Bundesnotarkammer auf Vorsorgevollmachten. Davon wurden im Jahr 2008 insgesamt 181.233 Vorsorgevollmachten neu registriert. Jahresbericht 2008 der Bundesnotarkammer, http://www.elrv.info/de/zvr/index.php (Stand: Dezember 2009).

${ }^{33}$ www.vorsorgeregister.de (Stand: Dezember 2009).

34 Siehe obige Ausführungen $\ 1$ A. II. 
bei unterschiedlichen Institutionen als Vordrucke erhältlich sind, ${ }^{35}$ und sich inhaltlich zumeist ausschließlich auf die namentliche Nennung des Wunschbetreuers beschränken. Die vorliegende Arbeit soll die aufgezeigte Lücke schließen.

\section{B. Weiterer Gang der Untersuchung}

Im Folgenden soll untersucht werden, inwieweit der Einzelne Wünsche, die seine Betreuung betreffen, antizipiert formulieren und sie für die Entscheidungen des Gerichts und des Betreuers verbindlich vorgeben kann. Es ist zu untersuchen, ob und in welchem Rahmen dies mit dem Rechtsinstitut Betreuungsverfügung für die Einrichtung, Führung und Überwachung einer später eventuell erforderlichen Betreuung möglich ist.

In $\int 2$ dieser Arbeit wird zunächst die Möglichkeit untersucht, mit Hilfe der Betreuungsverfügung das Recht auf Selbstbestimmung antizipiert für eine mögliche Betreuung auszuüben, indem diese durch die Wünsche und Vorstellungen des Betroffenen individuell ausgestaltet wird. Zur Verdeutlichung der Rechtsfragen soll dabei an den grundlegenden Stellen der Vergleich zum österreichischen Sachwalterrecht gezogen werden. Die $\iint 268 \mathrm{ff}$. des Österreichischen Allgemeinen Bürgerlichen Gesetzbuchs (ABGB) ${ }^{36}$ wurden 1983 neu gefasst und galten als Vorbild bei der Entwicklung des deutschen Betreuungsrechts der $\iint 1896$ ff. ${ }^{37}$ Ein Vergleich wird jedoch nur dort gezogen, wo sich Unterschiede in den rechtlichen Regelungen ergeben, um die einzelnen Besonderheiten im deutschen Recht herauszuarbeiten. Er bietet sich an, weil die Rechtsordnungen eine wechselseitige Vorbildfunktion hatten. Das Sachwalterrecht hatte für das deutsche Betreuungsrecht Vorbildcharakter, kannte selbst aber ursprünglich kein Äquivalent zur Betreuungsverfügung. Die Sachwalterverfügung wurde erst später nach dem Vorbild der deutschen Betreuungsverfügung in das österreichische ABGB eingeführt.

Aus dieser Untersuchung sind die Voraussetzungen der Betreuungsverfügung sowie die Wirkung in ihr enthaltener antizipiert geäußerter Wünsche zu entwickeln. Im Anschluss werden in den folgenden Paragraphen der gesetzlich mögliche Einfluss des Einzelnen auf die Auswahl des Betreuers ( $(3)$, das allgemeine Verfahren der Betreuerbestellung ( $\left(\int_{4}\right)$ sowie die Führung der Betreuung ( $\left(\int_{5}\right)$ mittels der Betreuungsverfügung und die Folgen ihrer Missachtung ( $\mathbb{S} 6)$ untersucht, bevor in $\int 7$ die wichtigsten Ergebnisse der Arbeit zusammenzufassen sind.

\footnotetext{
35 Vgl. nur „Vorsorgevollmacht und Betreuungsrecht“ Mecklenburg Vorpommern, Justizministerium Schwerin 2003; „Wie kann ich vorsorgen?“ Freistaat Thüringen Justizministerium, Erfurt 2008; „Das Betreuungsrecht“ Niedersachsen Justizministerium, Hannover 2005.

${ }^{36}$ Die einzelnen Paragrafen des ABGB sind abrufbar unter: http://www.ris.bka.gv.at/Bundesrecht/ (Stand: Dezember 2009).

${ }^{37}$ Mit dem Sachwalterrechts-Änderungsgesetz (SWRÄG), in Kraft seit dem 01.07.2007, wurde 2006 die erste große Novellierung des Sachwalterrechts vorgenommen, vgl. Erläut. RV 1420

BlgNr. XXII. GP 12.
} 



\section{$\$ 2$ Betreuungsverfügung als Instrument zur Ausübung des Selbstbestimmungsrechts}

In den Situationen der eigenen Hilflosigkeit ist der Einzelne den Entscheidungen Dritter ausgesetzt. Fraglich ist, ob die Betreuungsverfügung dabei hilft, diese Entscheidungen Dritter nach den eigenen Wünschen und dem eigenen Wohl zu gestalten. Es ist zu untersuchen, ob die Betreuungsverfügung dem Einzelnen die Möglichkeit bietet, das Selbstbestimmungsrecht für die mögliche eigene spätere Betreuung antizipiert auszuüben.

\section{A. Verfassungsrechtliche Grundlagen des Selbstbestimmungsrechts}

Das Selbstbestimmungsrecht bildet den Kern der durch das Grundgesetz (GG) in Art. 1 geschützten Menschenwürde. ${ }^{38}$ Menschenwürde und Selbstbestimmung steht jedem Menschen in gleicher Weise zu, auch Alten, Kranken und Behinderten, vgl. Art. 1 Abs. 1, Art. 3 Abs. 1 S. 1 GG. ${ }^{39}$ Das von der Menschenwürde umfasste Selbstbestimmungsrecht des Einzelnen bezieht sich auf die Eigenverant-

\footnotetext{
38 BGHZ 154, 205, 210; Dreier/Dreier Art. 1 GG Rn. 137.

${ }^{39}$ BK/Lorenz Art. 1 GG Rn. 381; Epping/Hillgruber Art. 1 GG Rn. 3 f.; BVerfGE 52, 131 ff.
} 
wortlichkeit in Lebensentscheidungen und den Kern individueller Selbstdarstellung nach außen. ${ }^{40}$ Träger des Rechts zur Selbstbestimmung ist gem. Art. 1 Abs. 1 GG jede natürliche Person von Beginn ihrer Existenz an. ${ }^{41}$ Es bedarf aber der Ausübung des Rechts, d.h. irgendeiner Form der Umsetzung im Rechtsverkehr. ${ }^{42}$

Der Wille des Betroffenen ist damit auch aus verfassungsrechtlichen Gründen grundsätzlich zu beachten. Jede Beschränkung bedarf daher einer Rechtfertigung. $\mathrm{Zu}$ rechtfertigen ist damit nicht die privatautonome Gestaltungsmöglichkeit mittels der Betreuungsverfügung, sondern ihre Beschränkung und ihre Grenzen.

\section{B. Ausübung des Selbstbestimmungsrechts im Betreuungsrecht gem. $\int \mathbb{S} 1896 \mathrm{ff}$.}

I. Betreuung als Fremd- bzw. Selbstbestimmung

Die Frage, ob ein gesetzlicher Vertreter das Selbstbestimmungsrecht eines anderen ausüben kann, wird schon ebenso lange wie kontrovers geführt. ${ }^{43}$ Dabei geht es allein um die Frage der Rechtsausübung. Mittlerweile ist anerkannt, dass das Recht auf Selbstbestimmung unabdingbar einer Person zusteht. Jeder Mensch hat daher das Recht, seine eignen Angelegenheiten frei und ohne Einmischung Dritter - insbesondere der von staatlicher Stelle - zu regeln.

Nach herrschender Meinung kann der gewillkürte Stellvertreter das Selbstbestimmungsrecht eines anderen ausüben. ${ }^{44}$ Die Vertretungsmacht des Stellvertreters beruht dabei auf dem Willensakt des Vollmachtgebers. Ein gesetzlicher Stellvertreter hingegen werde kraft Gesetzes und nicht durch den Willensakt des Vollmachtgebers legitimiert. ${ }^{45}$ Er treffe also eigene und daher für den Betroffenen fremde Entscheidungen, bestimmt diesen somit fremd. ${ }^{46}$ Schon aus diesem Grund könne der gesetzliche Vertreter nicht das Selbstbestimmungsrecht eines anderen ausüben. ${ }^{47}$ Das Recht auf Selbstbestimmung bliebe nur erhalten, solange seine Ausübung durch den Betroffenen erfolge. Dies ist für Betreute häufig gerade nicht mehr möglich. Selbstbestimmung und Betreuung schließen sich daher auf den

\footnotetext{
40 Maunz/Dürig/Herdegen Art. 1 GG Rn. 80.

${ }^{41}$ Epping/Hillgruber Art. 1 GG Rn. 3, 4, 12.

42 Lipp S. 53 f.

43 Vgl. nur Müller-Freienfels FS Coing S. 395, 399 f.; MünchKommBGB/Schramm Vor \164 Rn. 67; grundlegend zur Stellvertretung Staudinger/Schilken Vorbem. SS 164 ff. Rn. 3 ff.

44 Gessaphe S. 181 ff.; Müller-Freienfels FS Coing S. 395, 399.

45 Gessaphe S. $181 \mathrm{ff}$.

46 Vgl. statt vieler MünchKommBGB/Schwab Vor \1896 Rn. 14, \1896 Rn. 130 ff., 』 1902 Rn. 1, jeweils m.w.N.; noch zum alten Recht: Müller-Freienfels FS Coing S. 395, 400; kritisch: Lipp

S. 40 ff., 118 ff.

47 Gessaphe S. $181 \mathrm{ff}$.
} 
ersten Blick aus. ${ }^{48}$ Rechtliche Betreuung wäre demnach reine Fremdbestimmung. Dass sie dies gerade nicht ist, ergibt sich bereits aus \1901 Abs. 2 S. 3 und \ 1897 Abs. 4 S. 3, wonach Betroffenenvorschlägen zu entsprechen ist.

II. Aufgaben und Ziele der Betreuung

Betreute können aufgrund psychischer Krankheit, körperlicher, geistiger oder seelischer Behinderung ihre Angelegenheiten ganz oder teilweise nicht besorgen, vgl. \1896 Abs. 1 S. 1. Nach dem Betreuungsrecht bezieht sich dies nicht auf die tatsächliche Besorgung ihrer Angelegenheiten, ${ }^{49}$ sondern nur auf die beschränkte oder unmögliche Wahrnehmung ihrer Rechte. Das gilt auch für das Selbstbestimmungsrecht. Es bedarf nicht nur der Ausübung sondern auch der Behauptung gegen Eingriffe Dritter. Das Betreuungsrecht kompensiert dieses Defizit des Betreuten durch zwei Grundziele: Zum einen soll der Betreuer verlorene Fähigkeiten der Rechtsausübung seitens des Betreuten kompensieren. Zum anderen soll er den Schutz der Rechte und Interessen des Betroffenen gewährleisten. ${ }^{50}$

Diese Ziele des Betreuungsrechts spiegeln sich in dem gesetzlich festgelegten Grundsatz der Erforderlichkeit wider: Demnach ist ein Betreuer nur für die Aufgabenkreise zu bestellen, in denen der Betroffene aufgrund geistiger, körperlicher oder seelischer Behinderung seine Angelegenheiten ganz oder teilweise nicht mehr besorgen kann, ${ }^{51}$ vgl. \ 1896 Abs. 1 S. 1, Abs. 2. Damit soll der Eingriff in die Rechte des Betroffenen minimal erfolgen und der Erhalt seiner vorhandenen Fähigkeiten maximal gefördert werden. Der Grundsatz der Subsidiarität ergänzt dieses Ziel: Die Einrichtung einer Betreuung ist nicht möglich, soweit die Angelegenheiten des Volljährigen durch einen Bevollmächtigten oder durch andere Hilfen, bei denen kein gesetzlicher Vertreter bestellt wird, ebenso gut wie durch eine Betreuung besorgt werden können, vgl. \ 1896 Abs. 2 S. 2.

\footnotetext{
${ }^{48}$ Gessaphe sieht in der Einrichtung der Betreuung die Beschränkung des Selbstbestimmungsrechts (S. 54 ff.).

${ }^{49}$ Tatsächliche Angelegenheiten des Betroffenen zu besorgen, wie z. B. der hygienischen Körperpflege, ist nicht Aufgabe der rechtlichen Betreuung, sondern z. B. der von Pflegediensten (vgl. nur BR-Drucks. 960/96 S. 33); HK-BUR/Bauer \ 1901 Rn. 6; Bienwald/Sonnenfeld/Hoffmann $\int 1901$ Rn. 1.

${ }^{50}$ BT-Drucks. 11/4528 S. 52 f., 59 ff., 125; Lipp S. 40 ff.; Gessaphe S. 181 f.; May/Geißendörfer/Simon/Strätling/Lipp S. 45.

${ }^{51}$ Lipp S. 14 f.; Staudinger/Bienwald Vorbem. zu $\iint 1896$ ff. Rn. 38.
} 


\section{Rechtliche Stellung des Betreuten}

Der Gesetzgeber wollte mit dem „Gesetz zur Reform des Rechts der Vormundschaft und Pflegschaft für Volljährige“" von $1992^{52}$ v.a. die Entrechtung der Betroffenen als grundlegenden Mangel des bisherigen Rechts beenden. ${ }^{53}$ Das Betreuungsgesetz ersetzte die bis dahin geltende (Gebrechlichkeits-) Pflegschaft, Entmündigung und Vormundschaft über Volljährige. Schutz der Rechte und verfahrensmäßigen Positionen der betroffenen kranken und behinderten Menschen ist Inhalt des Betreuungsrechts. ${ }^{54}$ Die Art und Weise der Betreuung soll individuell, persönlich und nicht anonym erfolgen, ${ }^{55}$ wobei die rechtlichen Bedürfnisse des Volljährigen im Mittelpunkt stehen. In Abkehr zum alten Recht hat die Bestellung eines Betreuers keine Auswirkungen auf die rechtliche Handlungsfähigkeit des Betreuten. ${ }^{56}$ Seine Geschäfts- und Einwilligungsfähigkeit bleiben bestehen. I.d.R. besteht somit eine doppelte Vertretungsbefugnis: einerseits kann der Betreute selber für sich entscheiden, andererseits hat auch der Betreuer Vertretungsbefugnis. Lediglich bei einer ,erheblichen Gefahr für die Person oder das Vermögen“ kann das Betreuungsgericht nach $\ 1903$ Abs. 1 S. 1 einen Einwilligungsvorbehalt des Betreuers anordnen. Soweit dieser reicht, bedürfen Willenserklärungen des Betreuten, die den Aufgabenkreis des Betreuers betreffen, seiner Einwilligung.

\section{Aufgaben, Handlungs- und Entscheidungsmaßstab des Betreuers}

Der Betreuer vertritt den Betreuten gerichtlich und außergerichtlich, vgl. \ 1902. Er ist in seinen Entscheidungen und seinem Handeln an die Wünsche und Vorstellungen des Betreuten gebunden, vgl. \ 1901 Abs. 2 und 3. Er trifft zwar eigene Entscheidungen, eine inhaltliche Entscheidungskompetenz steht ihm jedoch nur bedingt zu. Denn er setzt die Wünsche des Betreuten um und dessen Rechte durch. Dies gilt sowohl für die Bestellung des Betreuers als auch für die Führung der Betreuung, vgl. nur $\iint 1896$ Abs. 1a, 1897 Abs. 4, 1901 Abs. 3. Die Wünsche und Vorstellungen sind für den Betreuer Entscheidung- und Handlungsmaßstab, ${ }^{7}$ wenn sie in den bestellten Aufgabenkreis fallen, ${ }^{58}$ soweit dies dem Betroffenenwohl nicht zuwider läuft und dem Betreuer zumutbar ist, vgl. \1901 Abs. 3. Der Betreute soll im Rahmen seiner Fähigkeiten sein Leben nach seinen eigenen Wünschen und Vorstellungen selbst gestalten, vgl. \1901 Abs. 2 S. 2 a.E. Darin ist er

\footnotetext{
52 Das Gesetz zur Reform des Rechts der Vormundschaft und Pflegschaft für Volljährige (Betreuungsgesetz) wurde vom Bundestag am 25.04.1990 beschlossen, der Bundesrat stimmte am 01.06.1990 zu. Das Gesetz trat am 01.01.1992 in Kraft und führte den Begriff „Betreuung“ ein. Vgl. zum Gesetzgebungsverfahren ausführlich Bienwald BtG, 3. Aufl. Einführung Rn. 47 ff.

53 BT-Drucks. 11/4528 S. 49 ff., 52; Lipp S. 49 f.

54 Staudinger/Bienwald Vorbem. zu SS 1896 ff. Rn. 38 ff.; BT-Drucks. 11/4528 S. 52 ff.

55 BT-Drucks. 11/4528 S. 125.

56 Keilbach FamRZ 2003, 969.

$57 \mathrm{Vgl}$. dazu die Ausführungen unter $\int 2$ C. II.

58 Jurgeleit/Deusing \ 1901 Rn. 14; Bienwald/Sonnenfeld/Hoffmann \1896 Rn. 132; Lipp FS Bienwald S. 177, 180; Knittel S 1901 Rn. 8.
} 
vom Betreuer zu unterstützen. ${ }^{59}$ Die Erklärungen des Betreuten bedürfen der Umsetzung seines gesetzlichen Vertreters, des Betreuers.

Damit der Betreuer die erwünschte Unterstützung im Interesse und nach den Wünschen des Betreuten erbringen kann, muss sich der Betroffene ihm gegenüber in irgendeiner Form mitteilen. ${ }^{60}$ Eine ausdrückliche Äußerung ist nicht Voraussetzung für die Beachtlichkeit seiner Erklärungen. ${ }^{61}$ Somit kann der Betreute sich ebenso durch konkludentes Handeln erklären.

Die ausdrückliche gesetzliche Regelung in \ 1901 Abs. 3 zur rechtlichen Geltung aktueller und antizipierter Wünsche des Betreuten ist auch als Reaktion des Gesetzgebers auf die kritisierte Tatsache zu bewerten, dass unter dem alten Vormundschafts- und Pflegschaftsrecht der Wille der Betroffenen wenig geachtet wurde. ${ }^{62}$ Nach früherem Recht spielten Erklärungen des Volljährigen, der unter Vormundschaft und Pflegschaft stand, keine Rolle. ${ }^{63}$ Das Rechtsinstitut Betreuung soll im Gegensatz dazu allgemein nicht als Bevormundung im Sinne einer Fremdbestimmung, sondern als Hilfe und Sicherung der Selbstbestimmung angesehen werden. ${ }^{64}$

\section{Private Vorsorge als Ziel der Gesetzgebung}

Mit dem 1. Gesetz zur Änderung des Betreuungsrechts ${ }^{65}$ wurde das Bestreben gestärkt, private Vorsorge als vorrangiges Instrument vor der rechtlichen Betreuung $^{66} \mathrm{zu}$ verbreiten. ${ }^{67}$ Seitdem sind Betreuungsbehörden nach $\int 6 \mathrm{~S} .2$ Betreuungsbehördengesetz (BtBG), Betreuungsvereine nach \1908f Abs. 1 S. 1 verpflichtet, planmäßig über die Betreuungsverfügung und über die Möglichkeiten der privaten Vorsorge in Form der Vorsorgevollmacht zu informieren und die Aufklärung über diese Instrumente zu fördern. ${ }^{68}$ Darin zeigt sich das Interesse des Gesetzgebers an einem eigenständigen und bedachten Bürger, der sich frühzeitig Gedanken über seine private Vorsorge in Form der Vorsorgevollmacht als Alternative zur Betreuung oder der individuellen Ausgestaltung einer möglicherweise

\footnotetext{
59 BT-Drucks. 11/4528 S. 53; Staudinger/Bienwald, \ 1901 Rn. 25.

${ }^{60}$ HK-BUR/Bauer \1901 Rn. 34, \1897 Rn. 59; Knittel \$1901 Rn. 7; Palandt/Diedericbsen \ 1901 Rn. 5.

${ }^{61}$ Dies sieht Bauer zumindest für Wünsche bei ärztlichen Behandlungsmaßnahmen anders, vgl. HKBUR/Bauer \ 1901 Rn. 34; allgemein für Vorschläge des Betroffenen: Dodegge/Roth B. Rn. 51; BayObLG BtPrax 2000, 260; Peran MittRhNotK 1996, 285, 286.

${ }^{62}$ BT-Drucks. 11/4528 S.53, 67; Langenfeld S. 156; Taupitz JuS 1992, 9.

${ }^{63}$ Epple BWNotZ 1992, 27; BtG-DiskE S. 36 ff.

${ }^{64}$ BT-Drucks. 11/4528 S. 125.

65 Gesetz zur Änderung des Betreuungsrechts vom 25.06.1998, trat im Wesentlichen als 1. Betreuungsrechtsänderungsgesetz mit Wirkung zum 01.01.1999 in Kraft.

${ }^{66}$ Chauvistré S. 20.

${ }^{67}$ Zudem gab es weitere Änderungen im Verfahrensrecht, vgl. Bienwald BtG, 3. Aufl. Einführungen Rn. $48 \mathrm{ff}$.

${ }^{68}$ Siehe zur Beratung durch Betreuungsbehörden: Binschus ZfF 2000, 80 ff.; vgl. dazu ausführlich $₫ 2$ C. V. 3 .
} 
erforderlichen Betreuung mittels der Betreuungsverfügung macht. Das Gesetz nennt die Vorsorgevollmacht in $\int 1908 \mathrm{f}$ Abs. 1 Nr. 2a und $\int 6$ Abs. 1 S. 2 BtBG gleichstehend mit der Betreuungsverfügung. Während durch die Bevollmächtigung eines Dritten die Erforderlichkeit der Betreuung entfällt und gezielt vermieden werden soll, ermöglicht die Betreuungsverfügung die frühzeitige individuelle Ausgestaltung der Betreuung. Mit der Änderung dieser Regelungen sollte hauptsächlich die Alternative zur Betreuung in Gestalt der Vorsorgevollmacht aufgezeigt werden. ${ }^{69}$ Durch die Änderung in \1908f und \ 6 BtBG wurde die Betreuungsverfügung - mit dieser Bezeichnung - erstmals im Gesetz genannt.

Die Stärkung der privaten Vorsorge war ein Hauptziel der 2. Reform des Betreuungsrechts ${ }^{70}$ - wiederum mit dem Bestreben der Betreuungsvermeidung ${ }^{71}$. Die ursprüngliche Verpflichtung der Betreuungsbehörden nach $\int 6$ Abs. 1 BtBG zur Aufklärung über die Vorsorgevollmacht und die Betreuungsverfügung wurde um die Möglichkeit der Beglaubigung beider Instrumente durch die Behörde nach Abs. 2 - 6 erweitert. Diese Änderungen führten zur weiteren gesetzlichen Aufwertung der Instrumente. ${ }^{72}$

Bereits aus \ 1901 Abs. 2 S. 3 und \ 1897 Abs. 4 S. 3 ergibt sich, dass Betroffenenvorschlägen - also auch in einer Betreuungsverfügung formulierten Wünschen - zu entsprechen ist. Mit Einführung des Betreuungsrechts wurde dem Vorschlag des Betroffenen aufgrund dieser gesetzlichen Regelung Bedeutung und Verbindlichkeit zugeschrieben. ${ }^{73}$ Wenn die in der Betreuungsverfügung festgehaltenen Wünsche verbindlich sind, ist sie ein Instrument, die gesetzlich geregelte Betreuung privatautonom auszugestalten. Damit könnte sie ein hohes Maß an Selbstbestimmung in der Betreuung gewährleisten. Rechtliche Betreuung wäre dann schon deshalb keine Fremd-, sondern Selbstbestimmung. ${ }^{74}$

Weil Wünsche des Betreuten immer der Umsetzung durch den Betreuer bedürfen, birgt die Betreuung aus zwei Gründen die Gefahr der Fremdbestimmung: Zum einen könnte der Betreuer die Wünsche des Betreuten nicht beachten oder sie aufgrund subjektiver Wertung verändern. Zum anderen besteht die Gefahr, dass der Betreuer den - aus Sicht des Betroffenen unerwünschten - Einfluss durch Dritte nicht abwendet. In beiden Fällen wird das Leben des Betroffenen entgegen seiner Wünsche fremdbestimmt. Er kann es nicht so führen, wie er

${ }^{69}$ BT-Drucks. 15/2494 S. 15, 44.

70 Das 2. Betreuungsrechtsänderungsgesetz (2. BtÄndG) wurde vom Bundestag am 18.02.2005 verabschiedet. Der Bundesrat stimmte am 18.03.2005 zu (BR-Drucks. 121/05). Das 2. BtÄndG trat am 01.07.2005 in Kraft und wurde am 21.04.2005 veröffentlicht (BGBl. I S. 1073; BTDrucks. 15/4874).

71 Zweiter Themenkomplex des 2. BtÄndG war die Neuregelung des Rechts der Vergütung und des Aufwendungsersatzes. Die Abrechnung des Betreuers wurde von der Stundenvergütung auf die sog. Fallpauschale umgestellt (BT-Drucks. 15/4874).

72 Siehe dazu: Binschus ZfF 2000, 80 ff.

${ }^{73}$ BT-Drucks. 11/4528 S. 127; Vgl. dazu ausführlich unter \2 C. II. 1. und 2.

${ }^{74} \mathrm{Vgl}$. nur Lipp S. 48 ff. 
selbst bestimmt hat. Nicht nur die Ausübung seines Selbstbestimmungsrechts muss gewährt werden, es ist auch gegen Eingriffe Dritter zu schützen.

\section{Selbstbestimmungsrecht in der Betreuung}

Durch die Einrichtung einer Betreuung kommt der Staat seinem Fürsorgeauftrag nach und sichert zugleich das Selbstbestimmungsrecht des Betroffenen. Die Einrichtung einer Betreuung ist ein Eingriff in die Rechte des Betroffenen und bedarf daher der Rechtfertigung. ${ }^{75}$ Das Recht auf Selbstbestimmung des Betreuten wird zunächst gegenüber der Betreuung insgesamt dadurch gewahrt, dass ihre Einrichtung gegen seinen freien Willen nicht möglich ist, vgl. $\int 1896$ Abs. 1a. ${ }^{76}$ In Rahmen der Einrichtung und Führung der Betreuung wird das Selbstbestimmungsrecht dann dadurch gewährleistet, dass die Betreuung gem. \ 1901 Abs. 2, 3 und \1901a nach den Wünschen und Vorstellungen des Betroffenen zu gestalten ist und nur zu seinem Wohl erfolgen darf. Diese Ausrichtung auf das Betroffenenwohl sieht das Gesetz darüber hinaus in den einzelnen Regelungen des Betreuungsrechts vor, schützt somit vor Fremdbestimmung und ermöglicht Selbstbestimmung in der Betreuung. ${ }^{77}$

\section{Antizipierte Ausübung des Selbstbestimmungs- rechts mittels der Betreuungsverfügung}

\section{Inhalt der Betreuungsverfügung}

$\int 1901$ c S. $1^{78}$ bestimmt, dass Schriftstücke, in denen jemand für den Fall seiner Betreuung Vorschläge zur Auswahl des Betreuers oder Wünsche zur Wahrnehmung der Betreuung geäußert hat, an das Betreuungsgericht abzuliefern sind.

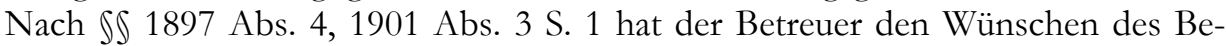
treuten zu entsprechen. Dies gilt gem. \ 1901 Abs. 3 S. 2 entsprechend \ 1897 Abs. 4 S. 3 auch für solche, die vor der Bestellung des Betreuers geäußert wurden.

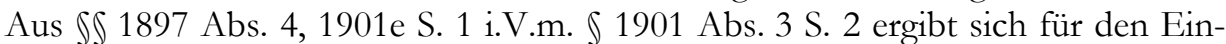
zelnen damit die Möglichkeit, antizipiert Wünsche in Bezug auf seine eigene Betreuung zu formulieren. Dies kann in Absehbarkeit der eigenen Betreuung oder unabhängig davon geschehen. Nach der gesetzlichen Formulierung handelt es sich

\footnotetext{
75 Die Frage, ob die gesetzliche Grundlage des mit der Betreuerbestellung staatlichen Eingriffs in die Rechtsstellung des Betroffenen hinreichend bestimmt sei, wurde vor der Einführung umfassend diskutiert, vgl. nur Bürgle NJW 1988, 1881, 1883; Pardey S. 3, 66, 86.

${ }^{76}$ Erfolgt die Einrichtung der Betreuung ohne oder gegen den Willen des Betroffenen, besteht besonders die Gefahr der Fremdbestimmung des Betreuten durch den Betreuer, vgl. BVerfGE 10, $302 \mathrm{ff}$.

${ }_{77}$ Diese dazu ausführlich Lipp S. 40 ff.; Gessaphe S. 54 ff.

${ }^{78}$ Durch die Normierung der Patientenverfügung wurden durch das 3. BtÄndG die \$S 1901a und $1901 \mathrm{~b}$ in das Gesetz eingefügt. Der vorherige \1901a wurde zu \1901c.
} 
im Rahmen der Betreuungsverfügung dabei nicht um Wünsche und Vorstellungen, die der Betreute während der Betreuung äußert, sondern ausschließlich um solche, die vor der Einrichtung der Betreuung gefasst wurden. Das Gesetz umschreibt somit das Rechtsinstitut Betreuungsverfügung, formuliert aber keine Wirksamkeitsvoraussetzungen.

\1901 Abs. 3 sieht keine thematische Beschränkung für antizipiert geäußerte Wünsche und somit für den Inhalt der Betreuungsverfügung vor. Danach können sich die Wünsche auf alle Angelegenheiten der Betreuung beziehen. Die Betreuungsverfügung enthält keine auf unmittelbare Rechtsgestaltung gerichtete Erklärung, ist also keine Willenserklärung. ${ }^{79}$ Mit ihr soll vielmehr Einfluss auf die Entscheidungen anderer genommen werden, nämlich auf die Entscheidungen des Betreuungsgerichts und des Betreuers. Damit stellt die Betreuungsverfügung eine besondere Form der Vorsorgeverfügung dar: Sie enthält keine antizipierte Regelung der Rechtsverhältnisse des Betreuten, sondern gestaltet die gesetzliche Betreuung als staatliche Rechtsfürsorge nach den eigenen Vorstellungen des Betreuten, d.h. privatautonom, aus.

Mit SS 1897 Abs. 4, 1901 Abs. 3 S. 2 wollte der deutsche Gesetzgeber Betreuungsverfügungen ausdrücklich anerkennen, ${ }^{80}$ auch wenn die Bezeichnung „Betreuungsverfügung" nur in $\ 1908 \mathrm{f} \mathrm{Nr} 2$ und in $\ 6$ Abs. 1 und 2 BtBG verwendet wird. Während das deutsche Recht die Betreuungsverfügung (nur) inhaltlich beschreibt, enthält das österreichische ABGB seit dem SachwalterrechtsÄnderungsgesetz (SWRÄG) $2006^{81}$ in $\ 279$ ABGB eine Legaldefinition der mit der Betreuungsverfügung vergleichbaren Sachwalterverfügung. Diese enthält „Wünsche der bebinderten Person, insbesondere solche, die sie vor Verlust der Geschäftsfähigkeit und Einsichts- und Urteilsfähigkeit geäußert bat."

Unter der behinderten Person versteht $₫ 273$ Abs. 1 ABGB die volljährige Person, die an einer psychischen Krankheit leidet oder geistig behindert ist. Dies ist nach dem Sachwalterrecht die sog. besachwaltete Person. Mit der gesetzlichen Formulierung von $\ 273$ ABGB hebt das Sachwalterrecht für die Sachwalterverfügung einige Besonderheiten hervor. Durch die Wortwahl ,insbesondere" wird deutlich, dass von der Sachwalterverfügung auch solche Wünsche erfasst sein können, die nach Eintritt der psychischen Krankheit oder geistigen Behinderung entstanden sind. Nach dem Wortlaut des $\ 1901$ c S. 1 werden von der Betreuungsverfügung hingegen nur solche Wünsche erfasst, die der Betroffene vor der Betreuung und somit vor dem Vorliegen einer psychischen Krankheit oder seelischen, körperlichen oder geistigen Behinderung gefasst hat. Im Unterschied zu der österreichischen Regelung wird in den $\$ \$ 1897$ Abs. 4, 1901, 1901c die Geschäfts-, Einsichts- und Urteilsfähigkeit des Betroffenen nicht aufgeführt.

\footnotetext{
${ }^{79}$ BT-Drucks. 11/4528 S. 127; Lipp Vorsorgeverfügungen \18 Rn. 5; Langenfeld S. 157 f.; MünchKommBGB/Schwab \$1901 Rn. 11, 13.

${ }^{80}$ Vgl. BT-Drucks. 11/4528 S. 128, 134.

${ }^{81}$ Vgl. zum SWRÄG Fn. 37.
} 


\section{Wirkung der Betreuungsverfügung}

Allgemein anerkannt ist, dass der Betroffene Wünsche zur Ausgestaltung seiner Betreuung äußern kann und diese rechtliche Anerkennung erfahren. Dies gilt auch für antizipiert geäußerte Wünsche und bezieht sich sowohl auf Wünsche in Bezug auf die Umstände der Einrichtung einer Betreuung als auch auf ihre Führung. Der Betreuer und das Betreuungsgericht haben sich an diese Vorgaben im Rahmen der gesetzlichen Grenzen zu halten. Damit bietet die Betreuungsverfügung für den einzelnen die Möglichkeit, die Entscheidungen des Betreuers und des Betreuungsgerichts individuell zu lenken und zu beeinflussen, auch wenn er in dem Zeitpunkt tatsächlich dazu selber nicht (mehr) in der Lage ist. Die Betreuungsverfügung ermöglicht daher auch im Rahmen der gesetzlichen Vorgaben individuelle Regelungen und Vorgaben für den Betreuer und das Betreuungsgericht zu schaffen und somit den gesetzlich bestimmten Entscheidungsspielraum von Betreuungsgericht und Betreuer einzuschränken. Sind diese antizipierten Wünsche des Betroffenen in der Betreuungsverfügung manifestiert, bietet das Gesetz mit diesem Institut für den Einzelnen eine Möglichkeit zur privatautonomen Ausgestaltung der gesetzlich geregelten Betreuung.

Welches die gesetzlichen Grenzen sind, in deren Rahmen die Betroffenenwünsche für die Entscheidungen des Gerichts und des Betreuers maßgeblich sind, ist bislang ungeklärt. Zu untersuchen ist dabei nicht nur die Grenze bzw. die Reichweite des rechtlich anerkannten Wunsches, sondern auch dessen Begründung. Die Reichweite der rechtlich anerkannten Betroffenenwünsche ist dem Gesetz zu entnehmen und wird in III. untersucht. Der Grund ihrer Bindung ist hingegen der gesetzlichen Bewertung des rechtlich anerkannten Wunsches abzuleiten. Diese Untersuchung ist in den folgenden Abschnitten 1. - 3. vorzunehmen.

\section{Wunsch des Betroffenen}

Das Betreuungsrecht ist auf den Schutz des Betreutenwohls ausgerichtet. Dieses bestimmt es anhand der Betreutenwünsche. Diese bewertet das Gesetz unterschiedlich: Nach \ 1901 Abs. 3 S. 2 i.V.m. S. 1 hat ein Betreuer früher geäußerten Wünschen zu entsprechen, es sei denn, der Betreute will an ihnen erkennbar nicht mehr festhalten. Nach \1901 Abs. 2 hat der Betreuer die Angelegenheiten des Betreuten so zu besorgen, wie es dessen Wohl entspricht. Dazu gehört die Möglichkeit, im Rahmen der Fähigkeiten des Betreuten dessen Leben nach seinen eigenen Wünschen und Vorstellungen zu gestalten. \ 1897 Abs. 4 nennt in S. 1 das Vorschlagsrecht des zu betreuenden Volljährigen für einen Betreuer, dem zu entsprechen ist. S. 2 spricht von dem Recht zum Vorschlag, eine bestimmte Person nicht zum Betreuer zu bestellen, auf das Rücksicht genommen werden soll. Beides gilt nach S. 3 auch für Vorschläge, die der Betreute vor dem Betreuungsverfahren gemacht hat. $\ 1896$ regelt in Abs. 1a, dass gegen den freien Willen des Volljährigen ein Betreuer nicht bestellt werden darf. 
Die beispielhafte Aufzählung der Normen zeigt die unterschiedliche Wirkung von geäußerten Wünschen vor oder in der Betreuung. Nach dem Gesetzeswortlaut ist allgemein festzustellen, dass ein Wunsch jede Äußerung des Betroffenen sein kann.

a) Einwilligungs- und Geschäftsfähigkeit des Betroffenen

Der Betroffene bleibt trotz der Einrichtung der Betreuung geschäftsfähig. ${ }^{82}$ Dies ergibt sich aus \ 1903. Demnach ordnet das Betreuungsgericht, soweit dies zur Abwehr einer erheblichen Gefahr für die Person oder das Vermögen des Betreuten erforderlich ist an, dass der Betreute zu einer Willenserklärung, die den Aufgabenkreis des Betreuers betrifft, dessen Einwilligung bedarf. Geschäftsunfähig ist gem. \ 104 nach Vollendung des siebten Lebensjahres nur derjenige, der sich in einem die freie Willensbestimmung ausschließendem Zustand krankhafter Störung der Geistestätigkeit befindet, sofern nicht der Zustand der Natur nach ein vorübergehender ist.

Für die Beachtlichkeit aktuell geäußerter Wünsche des Betroffenen in der Betreuung bedarf es keiner Geschäftsfähigkeit. ${ }^{83}$ Damit Wünsche auch im Rahmen von \ 1903 beachtlich. Die Betroffenenwünsche und -vorstellungen haben keine unmittelbar rechtsgestaltende Wirkung, sondern müssen dazu ggf. durch den Betreuer in eigener Verantwortung und Ausübung seiner Kompetenz umgesetzt werden. ${ }^{84}$

Beim Abfassen einer Betreuungsverfügung wird die Geschäftsfähigkeit des Verfassers regelmäßig (noch) vorliegen. Schließlich soll die Betreuungsverfügung gerade auch den Fall der eigenen Geschäftsunfähigkeit regeln. Auch die Wünsche der Betreuungsverfügung enthalten keine auf unmittelbare Rechtsgestaltung gerichtete Erklärungen, sondern sollen Einfluss auf die Entscheidungen des Gerichts und des Betreuers nehmen und bedürfen der Umsetzung. Die Geschäftsfähigkeit des Betroffenen bei der Abfassung seiner Betreuungsverfügung ist damit irrelevant. ${ }^{85}$

Die Betreuung berührt die Einwilligungsfähigkeit des Betreuten nicht. Seine Einwilligungsfähigkeit ist für die Beachtlichkeit der Betreutenwünsche ebenfalls

\footnotetext{
82 Müller S. 49 ff.; Palandt/Diederichsen \1901 Rn. 5; MünchKommBGB/Schwab \1897 Rn. 19.

83 BayObLG BtPrax 1993, 171; BayObLG NJW-RR 1997, 71; Peran MittRhNotK 1996, 285, 286; Palandt/Diederichsen \1901 Rn. 5; MünchKommBGB/Schwab \1897 Rn. 19; Damrau/Zimmermann $\$ 1901$ Rn. 9.

${ }^{84}$ BGHZ 154, 205, 211; BGH NJW 2005, 2385; Lipp FamRZ 2004, 317, 321 und FS Bienwald S. $177,178$.

85 BT-Drucks. 11/4528 S. 67, 127, 133; BayObLG NJW-RR 1997, 71; BayObLG BtPrax 1993, 171; Dodegge/Roth B. Rn. 51; Epple BWNotZ 1992, 27, 31; Perau MittRhNotK 1996, 285, 286; Langenfeld S. 157 f.; Lipp FS Bienwald S. 177, 178; Soergel/Zimmermann \$1901a Rn. 1; Keim A. Rn. 29; Palandt/Diederichsen \1901 Rn. 5; Bamberger/Roth/Müller \1901 Rn. 4; Erman/Roth \ 1901 Rn. 10; MünchKommBGB/Schwab \$ 1901 Rn. 11 \} 1 8 9 7 \text { Rn. 19; Hausmann/Hohloch/Röthel } Kapitel 14 Rn. 171, 174; Jurgeleit/Jurgeleit \1897 Rn. 33.
} 
unbeachtlich. Der verfassungsrechtliche Ausgangspunkt, so viel Autonomie wie möglich, und so wenig Heteronomie wie nötig und die Betonung des Persönlichkeitsschutzes führen dazu, während der Betreuung selbst Wünsche Einwilligungsunfähiger zu berücksichtigen. ${ }^{86}$ Für die Bindung an die antizipierten Vorschläge und Wünsche des Betroffenen in der Betreuungsverfügung nach $\iint 1897$ Abs. 4, 1901 Abs. 3 S. 1 und 2 kann es daher ebenfalls nicht auf seine Einwilligungsfähigkeit ankommen. ${ }^{87}$

Daher sind Wünsche auf bei Geschäftsunfähigkeit und Einsichtsunfähig bzw. bei \ 1903 grundsätzlich beachtlich.

Damit unterscheiden sich die Regelungen der SS 1896 ff. von den österreichischen Regelungen in \$S 279 Abs. 2 S. 1, 280 f. ABGB. Während der Sachwalterschaft kann die besachwaltete Person ${ }^{88}$ nach österreichischen Recht gem. \ 280 Abs. 1 ABGB innerhalb des Wirkungskreises des Sachwalters ohne dessen ausdrückliche oder stillschweigende Einwilligung rechtsgeschäftlich weder verfügen noch sich verpflichten. ${ }^{89}$ Dies gilt auch, wenn die besachwaltete Person tatsächlich einsichts- und urteilsfähig ist. ${ }^{90}$ Mit Eintritt der Rechtskraft des Beschlusses über die Sachwalterbestellung wird die behinderte Person im Wirkungskreis des Sachwalters in ihrer Geschäftsfähigkeit beschränkt. ${ }^{91}$

Nach \279 Abs. 1 S. 2 ABGB entfalten nur solche Wünsche der Sachwalterverfügung rechtliche Anerkennung, die vor Verlust der Geschäfts- und Einsichtsfähigkeit gefasst wurden. ${ }^{92} \mathrm{Nach} \int 281$ Abs. 1 ABGB hat der Sachwalter danach zu trachten, dass die behinderte Person im Rahmen ihrer Fähigkeiten und Möglichkeiten ihre Lebensverhältnisse nach ihren Wünschen und Vorstellungen gestalten kann. Demnach erfahren Wünsche, die der Besachwaltete in der Sachwalterschaft - und somit nach Verlust seiner Geschäfts- und Einsichtsfähigkeit - äußert, gegenüber in der Sachwalterverfügung antizipiert geäußerten Wünschen ein vergleichsweise geringes $\mathrm{Maß}$ an Berücksichtigung. ${ }^{93}$ \ 281 Abs. 1 ABGBG enthält die - dem deutschen Recht nachgebildete - Wunschbeachtungspflicht. ${ }^{94}$ Da das österreichische Recht jedoch eine ausdrückliche Unterscheidung in der Qualität

86 Spickhoff AcP Bd. 208 (2008), S. 345, 394 f.

87 Vgl. BT-Drucks. 11/4528 S. 67, 133; BayObLG FamRZ 1994, 322; Palandt/Diederichsen \ 1901

Rn. 5; Erman/Roth \ 1901 Rn. 10; MünchKommBGB/Schwab \ 1897 Rn. 19; Perau

MittRhNotK 1996, 285, 286.

88 Nach dem ABGB nennt man die unter Sachwalterschaft stehende Person den Besachwalteten.

${ }^{89}$ Koziol/Bydlinski/Bollenberger/Hopf $\$ 281$ ABGB Rn. 1.

${ }^{90} \mathrm{Zu}$ rechtsgeschäftlichen Verfügungen und Verpflichtungen bedarf sie der Einwilligung des Sachwalters (Koziol/Bydlinski/Bollenberger/Hopf $\$ 281$ ABGB Rn. 1).

${ }^{91} \mathrm{Koziol} /$ Bydlinski/Bollenberger/Hopf 281 ABGB Rn. 1; Kremzow noch zur ursprünglichen Regelung in Kremzow \$273a ABGB Anm. 1 (S. 55).

${ }^{2}$ So auch Barth/Ganner I. B. 2. b. (S. 57). Wenn auch mit umstrittener Wirkung, vgl. S 2 C. II. 2. c).

${ }_{93}$ Barth/Ganner B. 2. b (1) (S. 56 f.); Schwimann/Weitzenböck \$279 ABGB Rn. 4; Schauer ÖJZ 2007, 173, 178.

${ }^{94}$ Nach dem österreichischem Verständnis ist dies aber eher eine „Wunschermittlung“, vgl. Zierl $\S 281$ ABGB (S. 124). 
der Wünsche vor und nach Verlust der Geschäfts- und Einwilligungsfähigkeit trifft, hat die Wunschbeachtungspflicht des ABGB nicht die gleiche Bedeutung wie die des \1901 Abs. 2 und 3. Der Vergleich unterstreicht die Besonderheit der Regelung im deutschen Recht, nach der Wünsche des Betroffenen unabhängig von seiner Geschäfts- und Einwilligungsfähigkeit zu beachten sind. ${ }^{95}$ Dies gilt nicht nur für aktuell geäußerte Wünsche in der Betreuung. Vielmehr muss dies erst recht für das Abfassen einer Betreuungsverfügung gelten.

\section{b) Auslegung der Betreuungsverfügung}

Mittels der Auslegung wird beantwortet, was der Verfasser mit seiner Äußerung erreichen will. ${ }^{96} \mathrm{Ob}$ er dies erreicht, ist eine Frage der Umsetzung und Ausführung. Beides kann nicht ausschließlich dem Wortlaut der Betreuungsverfügung entnommen werden. ${ }^{97}$ Vielmehr ist der Inhalt der Äußerung mittels der allgemeinen Auslegungsregel des $\int 133$ zu ermitteln. ${ }^{98}$ Zwar handelt es sich bei den Äußerungen einer Betreuungsverfügung nicht um Willenserklärungen i.S.v. \133, sondern um Wünsche. Maßgeblich ist jedoch, dass festzustellen ist, was der Verfasser erklären wollte und was sein Wunsch ist. Dies ist mittels der Auslegungsmethoden von Willenserklärungen möglich. ${ }^{99}$ Analytisch ist zu fragen, was der Verfasser mit seiner Verfügung sagen wollte. ${ }^{100}$ Bei der Auslegung des antizipiert Geäußerten ist allein auf den Willen des Verfassers abzustellen, ${ }^{101}$ nicht auf den ihrer Adressaten oder Dritter. Diese sind nicht schutzwürdig. ${ }^{102}$ Dritte haben nur die Aufgabe, den Willen des Betroffenen zu ermitteln ${ }^{103}$ und zur Durchsetzung zu verhelfen ${ }^{104}$.

Es macht keinen Unterschied, ob der Wunsch vor langer Zeit oder aktuell vom Betroffenen geäußert wurde. Vielmehr ist entscheidend, dass der Inhalt der

${ }^{95}$ BtG-DiskE S. 123 ff.; BT-Drucks. 11/4528 S. 67, 127, 133; BayObLG BtPrax 1993, 171; BayObLG BtPrax 2002, 36, 37; BayObLG BtPrax 2002, 165, 166; BayObLG BtPrax 2003, 270; BayOLG BtPrax 2005, 35, 36; OLG Hamm BtPrax 1996, 189; OLG Frankfurt/Main BtPrax 1997, 123; Bienwald/Sonnenfeld/Hoffmann \1897 Rn. 53; Dodegge/Roth C. Rn. 125, 129, B. Rn. 5; MünchKommBGB/Schwab \1901 Rn. 11; Knittel』 1901 Rn. 7; Lipp S. 17 f. und S. 149 ff. und FS Bienwald S. 177, 178; Palandt/Diederichsen \$1901 Rn. 5; Langenfeld S. 157; Perau MittRhNotK 1996, 285, 286; Staudinger/Bienwald \$ 1901 Rn. 25; HK-BUR/Bauer \$1901 Rn. 34.

${ }^{96}$ Flume S. 291.

${ }^{97}$ Coeppicus Sachfragen S. 56; Lipp FS Bienwald S. 177, 181 und Patientenautonomie und Lebensschutz, S. 25; Bienwald/Sonnenfeld/Hoffmann \1901a Rn. 6; für die Auslegung einer Patientenverfügung: Roth JZ 2004, 494, 499; AG Frankfurt a.M. BtPrax 2002, 222, 223.

98 Zum Teil werden diese Grundsätze ausdrücklich nur dem Sinn nach angewandt, weil es sich bei der Betreuungsverfügung nicht um Willenerklärungen handele, vgl. Bienwald/Sonnenfeld/-

Hoffmann \1901a Rn. 6; vgl. allgemein Bydlinski S. 428 ff.

${ }^{99}$ Lipp Vorsorgeverfügungen \ 18 Rn. 30; Staudinger/Roth \ 157 Rn. 30.

${ }^{100}$ Lipp Patientenautonomie S. 24 f.

101 Roth JZ 2004, 494, 499.

102 Dabei gilt das Gleiche wie im Rahmen der Testamentsauslegung unter Heranziehung der Auslegungskriterien von \2259: Zimmermann Rn. 365.

103 Lipp Patientenautonomie S. 25.

104 Roth JZ 2004, 494, 499. 
Betreuungsverfügung dem gegenwärtigen Wunsch des Betroffenen entspricht. Da nach \1901 Abs. 3 S. 2 a.E. der aktuelle Wunsch zu ermitteln ist.

Wie im Rahmen der Testamentsauslegung sind zusätzlich die Grundsätze der ergänzenden Auslegung heranzuziehen. Es ist zu hinterfragen, wie der hypothetische Wille des Betroffenen bei Berücksichtigung der aktuellen Umstände wäre, bzw. wie dieser reagiert und agiert hätte. ${ }^{105}$ Der Einwand, mit hypothetischen Überlegungen den Betroffenen der Wirkung seines Willens zu berauben, artikuliert die Angst vor Missbrauch. Er kann aber nicht eine allgemein anerkannte Auslegungsmethode in Frage stellen, solange diese nicht zur rein spekulativ hypothetischen Überlegung wird. ${ }^{106}$

$\mathrm{Ob}$ es sich bei der Betroffenäußerung um ein allgemeines Werturteil oder einen konkreten Wunsch handelt, ist mittels der Auslegung zu ermitteln. So kann berücksichtigt werden, ob der Verfasser die Tragweite seiner Erklärung überblickt hat ${ }^{107}$ oder wie er damals unbekannte Tatsachen heute werten würde. ${ }^{108}$ Derart lässt sich analysieren, ob er sich weniger oder sehr intensiv und reflektiert mit dem Inhalt beschäftigt hat. ${ }^{109}$ Dieser Unterschied wird beim Vergleich eines selbst formulierten Textes und eines (angekreuzten) Formulartextes deutlich.

Bei dieser Art der Auslegung sind auch Informationen über den Betroffenen zu berücksichtigen, die über die Betreuungsverfügung hinaus bekannt sind. Ggf. sind dazu zusätzliche Informationen zu beschaffen. Insbesondere der bisherige Lebensumstand und die Wertvorstellungen des Betroffenen sind zu berücksichtigen.

Vor diesem Hintergrund ist der Inhalt einer Betreuungsverfügung auszulegen. Die Wirkung einer Betreuungsverfügung allgemein und die Verbindlichkeit der in ihr enthaltenen Wünsche speziell lässt sich deshalb nicht abstrakt und generell festlegen, sondern erst im Wege der Auslegung der konkreten Verfügung. ${ }^{10}$ Dabei wird deutlich, dass jede Verfügung einen anderen - individuellen - Inhalt hat, den allein der Verfasser bestimmt. Will er den Grad ihrer Verbindlichkeit bestimmen, muss er seine Wünsche entsprechend formulieren. Je weiter er die Inhalte der Betreuungsverfügung fasst und formuliert, umso geringer soll nach seinem Willen ihre Bindungswirkung sein und umgekehrt. ${ }^{111}$ Verfasst er einen sehr konkret formulierten Wunsch, will er ihn für den Betreuer als Anweisung bestimmen. Der BGH hat dies für Anweisungen in Bezug auf die medizinische Behandlung i.R.d. Patientenverfügung bestätigt. ${ }^{112}$ Für andere, nicht-medizinische Wünsche muss

\footnotetext{
105 Roth JZ 2004, 494, 499; Brox/Walker Rn. 201.

106 Roth JZ 2004, 494, 499; Lipp FamRZ 2004, 317, 320.

107 Coeppicus Sachfragen S. 56.

108 B ̈̈K NJW 1998, 3406, 3407; Coeppicus Sachfragen S. 56 f.

109 Coeppicus Sachfragen S. 56.

110 Anders: Taupitz A 121.

111 Lipp FS Bienwald S. 177, 181.

112 BGHZ 154, 205, 210, 211; bestätigend BGHZ 163, 195, 198.
} 
dasselbe gelten. ${ }^{113}$ Formuliert der Betroffene hingegen nur allgemein Lebenseinstellungen und Wertansichten, will er dem Betreuer nur einen Entscheidungsmaßstab an die Hand geben.

c) Wirkung der Betroffenenwünsche

aa) Innenwirkung

Der Wunsch des Betreuten hat innerhalb und außerhalb der Betreuung unterschiedliche Wirkung. Nach \1901 Abs. 3 S. 1 hat der Betreuer i.R. des ihm zugewiesenen Aufgabenkreises einem Betreutenwunsch zu entsprechen, soweit dieser dessen Wohl nicht zuwider läuft und die Umsetzung dem Betreuer zumutbar ist. Gem. S. 2 gilt dies auch für Wünsche, die der Betreute vor der Bestellung der Betreuung geäußert hat. Der Wunsch des Betreuten ist damit im Innenverhältnis bindend. Diese Bindung ist relativ, weil sie nur gegenüber dem Betreuer gilt. ${ }^{114}$ Sie wird absolut, wenn der Betreuer den Wunsch umsetzt. Um die Wünsche des Betreuten umsetzen zu können, muss der Betreuer diese auslegen und hat die ihm bekannten Vorstellungen des Betreuten und seine Lebensumstände mit in den Auslegungsprozess einzubeziehen. Er kann sich dabei nach \1837 Abs. 1 S. 1 vom Betreuungsgericht beraten lassen, dessen Aufgabe nach Abs. 2 u.a. gerade darin besteht, den Betreuer zur Einhaltung seiner Wunschbefolgungspflicht aus $\int 1901$ Abs. 3 S. 1 und S. 2 anzuhalten. ${ }^{115}$

Das Wohl des Betroffenen und die Zumutbarkeit für den Betreuer stellen die gesetzlich vorgegebene Grenze der Verbindlichkeit des Betroffenenwunsches dar. ${ }^{116} \mathrm{Ob}$ der Wunsch diesen oder einer anderen zwingenden gesetzlichen Regelung widerspricht, ist durch den Betreuer bzw. das Betreuungsgericht zu ermitteln.

\section{bb) Außenwirkung}

Der rechtlich anerkannte Wunsch des Betreuten hat keine eigene rechtsgestaltende Außenwirkung. Diese erfährt er erst, wenn der Betreuer ihn nach \1902 umsetzt, ${ }^{117}$ indem er eine eigene Willenserklärung abgibt. Für dieses Vertreterhandeln des Betreuers gelten die allgemeinen Vorschriften zur Stellvertretung nach

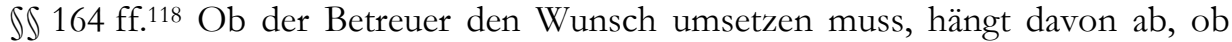
dieser für ihn nach \1901 Abs. 3 S. 1 im Innenverhältnis verbindlich ist. Ist dies

\footnotetext{
113 Lipp FS Bienwald S. 177, 181; Roth, JZ 2004, 494, 499; Berger JZ 2000, 797, 805.

114 So auch Barth/Ganner zum Wunsch in der Sachwalterverfügung; v.a. Ganner vertritt in der Frage der Verbindlichkeit der in der Sachwalterverfügung enthaltenen Wünsche einen ähnlich weit reichenden Ansatz, vgl. Barth/Ganner I. B. 2. b. (1) (S. 57) hingegen: Barth iFamZ 2006, 138, 141.

115 Soergel/Zimmermann $\int 1837$ Rn. 4.

116 Die Frage seiner konkreten Definition ist an dieser Stelle nicht maßgeblich und daher weiter unten zu behandeln, vgl. \ 2 C. III.

117 BGHZ 154, 205, 211; BGHZ 163, 195, 198; Taupitz A 121.

118 Jürgens/Kröger/Marschner/Winterstein Rn. 175.
} 
gegeben, bestimmt nach der Formulierung des \1901 Abs. 2 der Betroffene mittels seiner Wünsche, wie der Wunsch umgesetzt werden soll.

d) Zwischenergebnis

Ein Wunsch des Betroffenen kann jede Äußerung sein. An ihn sind gerade nicht die Voraussetzungen zu stellen, die für eine Willenserklärung im rechtsgeschäftlichen Bereich gelten. Was der Betroffene mit seinem Wunsch ausdrücken möchte, ist mittels Auslegung primär durch den Betreuer zu ermitteln. Dieser muss die Wünsche des Betreuten als dessen gesetzlicher Vertreter ermitteln und umsetzen. Erst dadurch erhalten sie rechtsgestaltende Wirkung. Ob der Betreuer den Wunsch umsetzen muss, hängt davon $a b$, ob dieser für ihn verbindlich ist. Dies ist in Abschnitt 2. zu untersuchen. Was gilt, wenn dies nicht gegeben ist, ist in Abschnitt 3. zu untersuchen.

\section{Geltung antizipiert geäußerter Wünsche in der Betreunng}

a) Vorrang des aktuellen Wunsches

Nach \ 1901 Abs. 2 und Abs. 3 S. 1 bilden bei der Einrichtung und Ausführung der Betreuung nach $\iint 1896$ ff. die aktuellen Wünsche und Vorstellungen des Betreuten den Entscheidungsmaßstab für den Betreuer und das Betreuungsgericht. Ist der Betreute nicht in der Lage, sich zu äußern und mitzuteilen, ist auf frühere Äußerungen - z.B. solche einer Betreuungsverfügung - zurückzugreifen. Liegen diese nicht vor, muss auf seinen mutmaßlichen Willen ausgewichen werden. ${ }^{119}$ Fehlen auch Anhaltspunkte zur Bildung eines mutmaßlichen Betroffenenwillens, kann nur nach objektiven Maßstäben entschieden werden. Ob diese im konkreten Fall dem (subjektiven) Willen des Betroffenen entsprechen, ist fraglich. Liegen keine individuellen Erklärungen des Betroffenen vor, besteht für ihn die Gefahr, durch die Entscheidungen des Betreuers fremdbestimmt zu werden. Diese Gefahr lässt sich mit Hilfe von im Voraus formulierter Wünsche für eine mögliche eigene Betreuung im Rahmen einer Betreuungsverfügung vermeiden. Dabei unterscheidet sich der Wunsch in einer Betreuungsverfügung gegenüber einer Willenserklärung in folgenden drei Punkten. Wünsche und Vorstellungen der Betreuungsverfügung werden zeitlich vor der Einrichtung einer Betreuung gebildet. Sie werden zudem in Ungewissheit ihrer Notwendigkeit formuliert. Der Betroffene verfasst Wünsche und Vorstellungen für eine Betreuung, deren Eintritt und Ausgestaltung unklar ist. Letztlich bedarf der in der Betreuungsverfügung enthaltene Wunsch zur Verwirklichung der Umsetzung durch Dritte.

\footnotetext{
119 Zur Ermittlung des mutmaßlichen Willens sind nahe stehende Personen, v.a. Angehörige und sonstige Vertrauenspersonen zu befragen, vgl. zum genauen Vorgehen ausführlich Spickhoff AcP Bd. 208 (2008), S. 345, 396. Für die Ermittlung des Patientenwillens ist dies seit dem 01.09.2009 in $\ 1901 \mathrm{~b}$ Abs. 2 festgelegt.
} 
Problematisch ist, wann ein derart früher erklärter Wille in der Betreuung Beachtung findet, und wann und warum er unbeachtlich bzw. von ihm abzuweichen ist.

b) Beachtlichkeit antizipiert geäußerter Wünsche

Vorgegeben ist zunächst, dass ein früherer Wunsch des Betroffenen in der Betreuung beachtlich ist.

Der Rechtsgedanke des $\int 130$ Abs. 2 unterstützt die Geltung antizipierter ÄuBerungen in der Betreuung. Direkt gilt $\int 130$ Abs. 2 für die Geschäftsunfähigkeit. Der Bundesgerichtshof überträgt dies auf die Einwilligungsunfähigkeit des Betroffenen. Demnach ändert sogar ,,die inzwischen eingetretene Einwilligungsunfäbigkeit [...] an der fortdauernden Maßgeblichkeit des früher erklärten Willens nichts. " 20 Dies ist auf die Betreuungsverfügung zu übertragen.

Das Recht auf Selbstbestimmung des Betroffenen sollte nach den Zielen der Gesetzgebung in der Betreuung besondere Beachtung finden, die Bedürfnisse und Ziele des Betreuten daher im Mittelpunkt stehen. Die Achtung seiner aktuellen Wünsche ist Ausfluss dessen. Konsequenterweise muss dies auch für antizipiert geäußerte Wünsche gelten. Sollen Wünsche, die unter Einfluss einer psychischen Krankheit oder körperlichen, geistigen oder seelischen Behinderung gebildet werden, während der Betreuung für das Betreuungsgericht und den Betreuer beachtlich sein und Geltung haben, muss dies erst recht für solche Wünsche gelten, die der Betroffene im Vollbesitz seiner geistigen Fähigkeiten gefasst hat. Übt der Betroffene sein Recht auf Selbstbestimmung aus und erklärt im Vorhinein seine Zustimmung oder Ablehnung einzelner Fragen der allgemeinen Lebensgestaltung, der Person des Betreuers und Ähnliches, ist er durch diese Äußerung zugleich mit der Last der Selbstverantwortung verbunden. ${ }^{121}$ Mit der vorherigen Festlegung seines Wunsches trägt der Betroffene das Risiko, diesen nicht mehr rechtzeitig ändern zu können. Diese Änderungslast ist Folge des Umstands, dass ein einmal erklärter Wunsch Geltung hat, bis dieser für andere ersichtlich geändert wurde, ${ }^{122}$ bzw. falls er dazu nicht mehr in der Lage ist, konkrete Anhaltspunkte dafür bestehen, dass er seinen Wunsch geändert hat. ${ }^{123} \mathrm{Nach}$ dem $\mathrm{BGH}$,gebietet die Würde des Menschen, sein in einwilligungsfähigem Zustand ausgeübtes Selbstbestimmungsrecht auch dann noch zu respektieren, wenn er zu eigenverantwortlichem Entscheiden nicht mehr in der Lage ist [...]. Ist der Patient im Zeitpunkt der Maßnahme nicht einwilligungsfähig, so gilt: Eine frühere Willensbekundung, mit welcher der Patient seine Einwilligung in Maßnabmen der in Frage

\footnotetext{
120 BGHZ 154, 205, 210 f.

121 Lipp Patientenautonomie S. 24; Taupitz A 13; i.Ü. ist sie durch die jederzeitige Widerrufsmöglichkeit begrenzt, vgl. $\int 2$ C. II. 2. d).

122 Roth JZ 2004, 494, 497; Lipp FamRZ 2004, 317, 320.

123 Grundlegens dazu die Grundsätze der BÄK, Ziff. IV NJW 1998, 3406 f. und Handreichungen der BÄK, DÄBl. 1999, A-2720 f.; Eisenbarth S. 67; Baumann/Hartmann DNotZ 2000, 594, 608 f.; Taupitz A 115.
} 
stehenden Art für eine Situation, wie sie jetzt eingetreten ist, erklärt oder verweigert hat, wirk.t, falls der Patient sie nicht widerrufen hat, fort. "124 In diesen Ausführungen bezieht der BGH sich inhaltlich auf die Einwilligung in medizinische Maßnahmen. Ihre Bindung begründet er mit dem Selbstbestimmungsrecht. Dieses erlaubt nicht nur vorherige Entscheidungen in Bezug auf medizinische Fragen. Vielmehr berechtigt das Recht auf Selbstbestimmung den Einzelnen dazu, alle Angelegenheiten des eigenen Lebens im Vorfeld selbst zu entscheiden. ${ }^{125}$ Die in der Betreuungsverfügung festgehaltenen Wünsche des Betreuten sind daher bindend. Sie zu befolgen steht nicht im Ermessen des Gerichts.

Obwohl das österreichische ABGB mit \ 279 Abs. 1 S. 2 ABGB eine Legaldefinition der Sachwalterverfügung getroffen hat, ist die Verbindlichkeit der in ihr enthaltenen antizipiert geäußerten Wünsche streitig. Das SWRÄG verfolgte unter anderem die Stärkung der Besachwaltetenrechte ${ }^{126}$ und damit ähnliche Ziele wie das Betreuungsrecht. ${ }^{127}$ Mit der gesetzlichen Normierung der Sachwalterverfügung im Jahr 2006 sollte der Wunschbeachtung bzw. der Autonomie der betroffenen Person als Ausdruck des Selbstbestimmungsrechts Rechnung getragen werden. ${ }^{128}$ Nach dem Wortlaut des \281 Abs. 2 ABGB hat der Sachwalter „Wünsche zu berücksichtigen", wohingegen \1901 Abs. 3 verlangt, dass der Betreuer den Betroffenenwünschen „₹и entsprechen hat". Übereinstimmend mit der Erläuterung zum SWRÄG versteht darunter der überwiegende Teil der Literatur, dass die Berücksichtigung der in der Sachwalterverfügung enthaltenen „Empfehlung im pflichtgebundenen Ermessen des Gerichts und des Sachwalters liegt. "129 Die Wünsche seien nicht bindend, sondern nur zu berücksichtigen. ${ }^{130}$ Wenige sehen durch $\ 279$ Abs. 1 S. 2 ABGB Gericht und Sachwalter grundsätzlich an die Erklärung gebunden. In letzterem Fall entfällt jedes Auswahlermessen für die Auswahl des Sachwalters. ${ }^{131}$ Dann hätten die Wünsche der Sachwalterverfügung die gleiche Wirkung wie die der Betreuungsverfügung.

\footnotetext{
124 BGHZ 154, 205, 205 und 210; sinngem. ebenso: Lipp Patientenautonomie S. 23 m.w.N. in Fn. 89 sowie Passive Sterbehilfe S. 43 und dort Fn. 37 m.w.N.; Taupitz A 41.

125 Lipp Vorsorgeverfügungen $\int 18$ Rn. 2.

126 Vgl. ausführlich: Ganner BtPrax 2007, 238 ff. und BtPrax 2008, 3 ff.; Maurer 275 ABGB Rn. 1 ff.; Barth iFamZ 2006, 138 ff.

127 Und die BtÄndG 1 und 2, vgl. dazu \2 B. II.

${ }^{128}$ Erläuterungen zum Sachwalterrecht 2006, S. 15; Koziol/Bydlinski/Bollenberger/Hopf \281 ABGB Rn. 1; Maurer \279 ABGB Rn. 15; Feil/Marent \279 ABGB neu Rn. 4.

${ }^{129}$ Erläuterungen zum Sachwalterrecht 2006, S. 13.

${ }^{130}$ Erläut. 1420 BlGNR XXII. GP S. 16; Koziol/Bydlinski/Bollenberger/Hopf $\$ 279$ ABGB Rn. 1; Maurer \179 ABGB Rn. 15; Feil/Marent \279 ABGB neu Rn. 4; Barth iFamZ 2006, 138, 141; Zierl\$279 ABGB (S. 65); so ist im Ergebnis wohl auch Weitzenböck zu verstehen, vgl. Schwimann/Weitzenböck \$ 279 ABGB Rn. 4.

131 Barth/Ganner I. B. 2. b. (S. 57).
} 
c) Voraussetzung für die Bindung

Der Wunsch des Betreuten ist jedoch nur rechtlich anerkannt, wenn er ernsthaft ${ }^{132}$ gefasst wurde. Dies ist anzunehmen, wenn er dauerhaft ${ }^{133}$ gebildet wurde. Nach verbreiteter Meinung dürfen an die Ernsthaftigkeit keine überspannten Anforderungen gestellt werden. ${ }^{134}$ Es müsse genügen, dass diese , zu deuten sei “ 135 . Des Weiteren muss es sich bei der Äußerung um einen bewussten, also einen vom natürlichen Willen getragenen ${ }^{136}$ und gerade nicht unbewusst entstandenen Wunsch handeln. Gerade bei geistig Behinderten wird die Erforschung des bewussten Willens den Richter vor nicht zu unterschätzende Probleme stellen. Der Wunsch muss frei von Einfluss Dritter ${ }^{137}$ gefasst sein, also eigenständig und aus eigenem Antrieb. Bei diesbezüglichen Zweifeln ist die eigenständige Entscheidungsfassung ggf. im Rahmen einer erneuten Anhörung zur prüfen. Letztlich darf der Wunsch keinem Irrtum unterliegen und nicht in einem die freie Willensbildung ausschließenden Zustand verfasst worden sein.138 Das Gesetz stellt diese Voraussetzungen jedoch weder direkt als Voraussetzung an den aktuellen Wunsch des Betroffenen, noch an seinen antizipiert geäußerten. Vielmehr überträgt es die Prüfung, was der maßgebliche Wunsch des Betroffenen ist und ob dieser bewusst geäußert, ernsthaft und frei von Irrtum und Einfluss Dritter gefasst wurde dem Betreuungsgericht und Betreuer. ${ }^{139}$

Diese Anforderungen stellen sicher, dass der antizipiert geäußerte Wunsch dem Willen des Betroffenen entspricht und schützen ihn daher vor einer ver-

132 OLG Hamm FamRZ 1996, 1372; BayObLG FamRZ 1999, 53; BayObLG BtPrax 2000, 260; BayObLG BtPrax 2002, 36, 37; BayObLG BtPrax 2002, 165, 166; BayObLG BtPrax 2003, 270; BayObLG BtPrax 2005, 35, 36; Dodegge/Roth B. Rn. 51; HK-BUR/Bauer $\ 1897$ Rn. 59a, 59b; Soergel/Zimmermann \1897 Rn. 31; Staudinger/Bienwald \$ 1897 Rn. 28; Bienwald/Sonnenfeld/Hoffmann \1897 Rn. 53; Damrau/Zimmermann \1897 Rn. 32.

133 Jürgens/Jürgens \1897 Rn. 14; BayObLG BtPrax 2005, 35, 36; BayObLG BtPrax 2003, 270; Dodegge/Roth B. Rn. 51.

134 OLG Hamm FamRZ 1996, 1372, 1372 f.; zum Teilwerden die zusätzlichen Kriterien (eigenständig, dauerhaft und unabhängig vom Einfluss Dritter) auch als Ausgestaltung bzw. Voraussetzung der Ernsthaftigkeit verstanden, vgl. HK-BUR/Bauer \$1897 Rn. 59b oder BayObLG BtPrax 2003, 270 und BayObLG BtPrax 2005, 35, 36 nach denen der Wunsch auf einer eigenständigen Willensbildung beruht, soweit er dauerhaft und unabhängig vom Einfluss Dritter entstanden ist.

135 Dodegge/Roth B. Rn. 51; Soergel/Zimmermann \1897 Rn. 31.

136 BayObLG BtPrax 2000, 260; BayObLG BtPrax 2002, 36, 37; BayObLG BtPrax 2002, 165, 166 ; Dodegge/Roth B. Rn. 51; HK-BUR/Bauer 1897 Rn. 59a; Perau MittRhNotK 1996, 285, 286; Soergel/Zimmermann \1897 Rn. 31; Larenz/Wolf 24 Rn. 3; Bartolomeycrik FS Ficker S. 51, 54 ff. 137 Dodegge/Roth B. Rn. 51; MünchKommBGB/Schwab \1897 Rn. 23; Jürgens/Jürgens \1897 Rn. 14; BayObLG BtPrax 2003, 270, BGH NJW 1970, 1680, 1681; BayObLG FamRZ 2001, 1555, 1556; Palandt/Diederichsen \ 1897 Rn. 17; OLG München RPfleger 2006, 651, 652; BayObLG NJW-RR 1997, 69, 70; BayObLG BtPrax 2002, 36, 37; OLG Düsseldorf BtPrax 1995, 108, 109; BayObLG BtPrax 2005, 35, 36; Dodegge/Roth B. Rn. 51; Soergel/Zimmermann \1897 Rn. 31; Staudinger/Bienwald \$1897 Rn. 28; Bienwald/Sonnenfeld/Hoffmann \1897 Rn. 53.

138 OLG Düsseldorf FamRZ 1998, 1064, 1066.

${ }^{139}$ Lipp Vorsorgeverfügungen $\ 18$ Rn. 13 m.w.N. 
fälschten Definition seiner Wünsche. Die Gefahr der Fremdbestimmung wird damit gebannt. Betreuer und Gericht haben dadurch zudem Anhaltspunkte zur Auslegung eines rechtlich anerkannten Betroffenenwunsches.

d) Fehlender Widerruf

\ 1901 Abs. 3 S. 2 spricht von Wünschen, die der Betreute vor der Betreuung geäußert hat und denen der Betreuer in der Betreuung entsprechen soll. Nach $\int 1897$ Abs. 4 S. 3 a.E. ist einem positiven Vorschlag des Betroffenen in Bezug auf die Betreuerauswahl nach S. 1 zu entsprechen, bzw. nach S. 2 auf die Ablehnung eines potentiellen Betreuers Rücksicht zu nehmen. Nach beiden Vorschriften gelten die Wünsche des Betroffenen, ,es sei denn, dass er an diesen Vorschlägen erkennbar nicht festhalten will. "Danach wird gesetzlich vermutet, dass der Betreute an antizipierten Wünschen festhalten will, solange nichts Gegenteiliges bekannt ist. Zeitablauf beeinträchtigt die Wirksamkeit seiner Wünsche demnach nicht. ${ }^{140}$

Der Willensvorrang des Betreuten nach \ 1901 Abs. 3 S. 2 bedeutet nach herrschender Meinung auch, dass aktuellen Äußerungen immer Vorrang zukommt. ${ }^{141}$ Widersprechen aktuelle den früheren Äußerungen, gelten sie als Widerruf und gehen nach \ 1901 Abs. 3 den früheren vor. ${ }^{142}$ Nach der gesetzgeberischen Begründung sollte auf diese Weise eine Selbstbindung des Betroffenen durch Abfassen einer Betreuungsverfügung vermieden werden. ${ }^{143}$ Nach der Begründung des Regierungsentwurfs begründe die fehlende Widerrufsmöglichkeit früherer Äußerungen die geringe Verbreitung der Betreuungsverfügung. ${ }^{144}$ Die endliche Festlegung führe zur Angst, sich selbst zu binden und damit eine spätere Änderung der eigenen Meinung unmöglich zu machen.

Nach dem österreichischen \279 ABGB finden in der Sachwalterverfügung nur solche antizipiert geäußerten Wünsche Berücksichtigung, die vor dem Verlust der Geschäfts-, Einsichts- und Urteilsfähigkeit geäußert wurden. ${ }^{145}$ Gleiches gilt für den Widerruf der Sachwalterverfügung: er setzt Geschäfts-, Einsichts- und Urteilsfähigkeit voraus. ${ }^{146}$ An den verbindlich gefassten Wunsch der Sachwalterverfügung sind nach Verlust der Geschäftsfähigkeit nicht nur Gericht und Sachwalter, sondern auch der Besachwaltete selber gebunden. Ein Widerruf seiner antizipiert geäußerten Wünsche ist dann für ihn nicht mehr möglich. ${ }^{147}$ Dies ist insbesondere in Bezug auf die - auch in $\int 281$ Abs. 1 und 2 ABGB enthaltene Wunschbeachtungspflicht des Besachwalteten bemerkenswert. Nach der Geset-

\footnotetext{
${ }^{140}$ Lipp Vorsorgeverfügungen \18 Rn. 32; Taupitz A 120.

141 BT-Drucks. 11/4528 S. 128; Bienwald/Sonnenfeld/Hoffmann \1901 Rn. 21; Soergel/Zimmermann \ 1901 Rn. 9.

142 Langenfeld S. 158; Martin S. 134; Damrau/Zimmermann S 1901 Rn. 13; anders: Müller S. 178.

143 BT-Drucks. 11/4528 S. 128.

144 BT-Drucks. 11/4528 S. 128.

145 Maurer $\$ 279$ ABGB Rn. 15.

146 Barth/Ganner I. B. 2. b. (2) (S. 58 f.).

147 Barth/Ganner I. B. 2. b. (2) (S. 58 f.).
} 
zesbegründung enthält der im Rahmen des SWRÄG von 2006 eingeführte \281 ABGB in Abs. 1 die allgemeine Richtschnur der Tätigkeit des Sachwalters, wonach dieser danach zu trachten hat, dass der Behinderte sein Leben grundsätzlich frei nach seinen eigenen Wünschen und Vorstellungen gestalten kann. ${ }^{148} \mathrm{Nach}$ der Literatur soll $\ 281$ Abs. 1 und 2 ABGB dem $\ 1901$ Abs. 3 entsprechen. ${ }^{149}$ Das deutsche Recht stellt im Vergleich jedoch geringere Anforderungen an einen rechtlich beachtlichen Wunsch des Betreuten. Nach \1901 Abs. 3 S. 2 gehen aktuelle Wünsche des Betreuten den antizipierten immer vor. Sämtliche Regelungen der Betreuungsverfügung kann der Betroffene daher jederzeit durch aktuelle anders ausgerichtete - Wünsche oder einen ausdrücklichen Widerruf aufheben. ${ }^{150}$ Betroffene, die aufgrund ihrer Krankheit oder Behinderung nicht (mehr) in der Lage sind, sich ausdrücklich zu artikulieren, können ihren Willen nach deutschem Recht durch eindeutiges Verhalten konkludent widerrufen. Bloße Reflexhandlungen bedeuten keinen Widerruf, 151 weil sie nicht willensgetragen sind. Vielmehr bedarf es eines erkennbaren Handelns oder Äußerns, aus dem die Meinungsänderung in Bezug auf seine geäußerten antizipierten Wünsche deutlich wird. Die konkreten Anforderungen an einen Widerruf müssen gleich denen eines antizipierten Wunsches des Betroffenen sein, der in der Betreuung Geltung hat. ${ }^{152}$ Demnach muss ein Widerruf des Betroffenen bewusst, ernsthaft, frei von Irrtum und Einfluss Dritter gebildet worden sein und ist nach deutschem Recht ausdrücklich oder konkludent zu jedem Zeitpunkt möglich. ${ }^{153}$ Geschäfts- oder Einwilligungsfähigkeit bedarf es nicht. ${ }^{154}$ Durch die inhaltlich hohen Anforderungen eines Widerrufs nach dem $A B G B$ ist dieser für den Besachwalteten hingegen gerade nicht jederzeit möglich. ${ }^{155}$ Durch die in $\ 281$ ABGB verankerte Wunschbeachtungspflicht des Sachwalters ist ein Widerruf des in der Sachwalterverfügung festgehaltenen antizipierten Wunsches durch einen entgegenstehenden aktuellen Wunsch aber letztlich doch möglich.

\footnotetext{
148 RV 1420 XXII. GP S. 18.

${ }^{149}$ Vgl. nur Erläut. 1420 BIGNR XXII. GP 16 S. 5, 20; Barth/Ganner I. C. 5. (S. 89).

150 OLG Düsseldorf FamRZ 1995, 894, 895; Epple BWNotZ 1992, 27, 30; Hausmann/Hohloch/Röthel Kapitel 14 Rn. 173 f.; Staudinger/Bienwald \1897 Rn. 28.

151 Berger JZ 2000, 797, 805.

152 In Bezug auf die Ernsthaftigkeit: Mayer S. 134.

153 Rudolf/Bittler \3 Rn. 24; Dodegge/Roth C. Rn. 78,129; Lipp FS Bienwald S. 177, 192 und Vorsorgeverfügungen \18 Rn. 32 f. ; Epple S. 27, 30 f.; Jürgeleit/Jürgeleit \ 1897 Rn. 35.

154 Zimmermann Rn. 366.

${ }_{155}$ Barth/Ganner sprechen zwar von der jederzeitigen formlosen Widerruflichkeit, dies aber nur bei bestehender Geschäfts-, Einsichts- und Urteilsfähigkeit, vgl. I. B. 2. b. (2) (S. 59). Bei diesen Anforderungen ist ein Widerruf aber gerade nicht jederzeit möglich.
} 
e) Zwischenergebnis

In der Betreuungsverfügung enthaltene, antizipiert gefasste Wünsche erfahren rechtliche Anerkennung, wenn sie bewusst, ernsthaft und frei von Irrtum und Einfluss Dritter gebildet wurden. Ob sie diesen Voraussetzungen entsprechen, ist von Betreuer und Gericht zu prüfen. Ist dies gegeben, sind sie verbindlich und von Betreuer und Gericht zwingend zu beachten. Dies folgt aus $₫ 1901$ Abs. 3 S. 1 i.V.m. S. 2, \ 1897 Abs. 4 sowie der - durch den BGH ausdrücklich ausgesprochenen - Anerkennung des Selbstbestimmungsrechts. Ihre Verbindlichkeit besteht, solange kein Anhaltspunkt für einen aktuell davon abweichenden Willen oder einen Widerruf vorliegt, vgl. \1901 Abs. 3 S. 2. Für den Widerruf gelten in diesem Fall dieselben Anforderungen wie für einen verbindlichen Wunsch des Betroffenen.

\section{Entfallen der Bindung an den Betroffenenwunsch}

Der Betroffenenwunsch ist für den Betreuer zwar verbindlich, aber nicht in allen Fällen beachtlich. Die Beachtlichkeit, also die Frage, ob der Betreuer den Wunsch gem. \1901 umsetzen muss, ist für jeden Einzelwunsch gesondert zu prüfen. Erst wenn sie gegeben ist, hat der Betreuer den Wunsch umzusetzen. Dies gilt aber nur, wenn die Wünsche in den Aufgabenkreis des Betreuers fallen und nicht gegen gesetzliche Vorgaben verstoßen. ${ }^{156}$

Wie bereits aufgezeigt, entfällt die Bindung an den Wunsch des Betroffenen nicht, nur weil der Betroffene beim Verfassen geschäfts- oder einwilligungsunfähig war. ${ }^{157}$ Sie entfällt auch nicht dadurch, dass Betreuungsgericht oder Betreuer etwas Anderes für besser halten oder dem Betroffenen durch die Umsetzung des Wunsches ein Schaden droht, denn SS 1901 Abs. 3 S. 1, 1897 Abs. 4 S. 1 begründen keinen Vorrang des objektiven Wohls bzw. der objektiven Interessen des Betroffenen vor seinen subjektiven Wünschen. ${ }^{158}$ Denn \ 1901 Abs. 2 definiert das Wohl des Betreuten subjektiv. ${ }^{159}$ Die $\int S 1901$ Abs. 3 S. 1, 1897 Abs. 4 S. 1 räumen Betreuungsgericht und Betreuer daher auch kein freies Ermessen ein, Wunsch und Wohl gegeneinander abzuwägen. \1901 Abs. 3 S. 1 formuliert vielmehr ausdrücklich eine Grenze für die Beachtlichkeit der Betroffenenwünsche. Danach hat der Betreuer Wünschen des Betreuten zu entsprechen, soweit dies dessen Wohl nicht zuwider läuft und dem Betreuer zumutbar ist. Was dies konkret bedeutet ist in Abschnitt III. zu untersuchen.

\footnotetext{
156 Der Wunsch auf aktive Sterbehilfe ist daher schon wegen \216 Strafgesetzbuch unbeachtlich. 157 Vgl. S 2 C. II. 1. a).

158 So ist aber wohl Zimmermann zu verstehen (Soergel/Zimmermann \1901 Rn. 10); dagegen zu Recht: HK-BUR/Bauer $\$ 1901$ Rn. 52.

159 Vgl. dazu ausführlich \2 C. III. 1.
} 
a) Fehlender freier und eigenverantwortlicher Wille

Darüber hinaus sieht das Betreuungsrecht keine ausdrücklichen Regelungen zur Beachtlichkeit der Betroffenenwünsche vor. Erfolgt ihre Bildung nicht bewusst, ernsthaft, frei von Irrtum und Einfluss Dritter erfüllt sie nicht die Voraussetzungen, die an den verbindlichen Wunsch zu stellen sind. Er entfaltet dann keine Rechtsfolge und ist daher unbeachtlich. Davon zu unterscheiden ist der Wunsch, der im Zustand fehlender Eigenverantwortlichkeit gebildet wurde. Die Bindung dieser Wünsche ist umstritten. Die Problematik stellt wieder die Frage des Abbzw. Überwiegens der Selbstbestimmung gegen die Fremdbestimmung dar. Bei der aktiven Hinderung der Umsetzung eines Betroffenenwunsches durch den Betreuer besteht ein großes Maß an Fremdbestimmung. Die schlichte Weigerung, einem Wunsch nachzukommen, beinhaltet ein geringeres $\mathrm{Maß}$ an Fremdbestimmung. Zu bestimmen ist, wann Fremdbestimmung erforderlich ist.

Die Bestellung des Betreuers nimmt dem Betroffenen nicht das verfassungsrechtlich garantierte Recht auf Freiheit zum Risiko. ${ }^{160}$ Für die Entscheidungen des Betreuers und des Betreuungsgerichts in Bezug auf die Beachtlichkeit der Wünsche müssten daher die gleichen Anforderungen gelten wie bei der Bestellung eines Betreuers gegen den Willen des Betroffenen nach \1896 Abs. 1 und 1a. ${ }^{161}$ Der Maßstab zur Beachtlichkeit der Betroffenenwünsche ist demnach die in Krankheit oder Behinderung gründende fehlende Eigenverantwortlichkeit. ${ }^{162}$ Sind die Wünsche Ausdruck einer psychischen Krankheit oder einer Behinderung und daher nicht mehr eigenverantwortlich gebildet, entfällt die Bindung an den Wunsch.

\section{b) Drohende Selbstschädigung}

Die Beachtung der Betroffenenwünsche kann zur objektiven Selbstschädigung des Betroffenen führen. Auch in dieser Frage gilt das verfassungsrechtlich garantierte Recht auf Freiheit zum Risiko. ${ }^{163}$ Fremdbestimmung ist demnach erst erforderlich, wenn sich der Betreute aufgrund seiner fehlenden Eigenverantwortlichkeit zu schädigen droht. Erst dann ist sie aber auch erlaubt. Der Betreuer darf die selbstschädigenden Wünsche daher nur dann unbeachtet lassen, wenn sie auf fehlender Eigenverantwortlichkeit basieren und der Betreute sich durch die Verwirklichung dieser Wünsche schädigen würde. Der Schaden muss dabei nicht erheblich oder an bedeutenden Rechtsgütern einzutreten drohen. Denn es geht allein um die Entstehung des Wunsches.

${ }^{160}$ Lipp Vorsorgeverfügungen \18 Rn. 21; MünchKommBGB/Schwab 1901 Rn. 14; HK-

BUR/Bauer $\$ 1901$ Rn. 43; BVerfGE 58, 208, 225 f.; BVerfG BtPrax 1998, 144, 145.

${ }_{161}$ So auch Lipp S. 138 f., 155 f. und Vorsorgeverfügungen $\ 18$ Rn. 21; Spickhoff AcP Bd. 208 (2008), S. $345,394 \mathrm{f}$.

162 Anders: Taupitz A 121.

163 BVerfGE 58, 208 ff. 
c) Zwischenergebnis

Das Selbstbestimmungsrecht erlaubt die Nichtbeachtung eines Betroffenenwunsches nur, wenn er auf einer krankheits- oder behindertenbedingt fehlenden Eigenverantwortlichkeit beruht bzw. durch seine Umsetzung der Betroffenen sich selbst zu schädigen droht. Im Übrigen ist auch ein selbstschädigender Wunsch beachtlich, solange er bewusst und gewollt, eigenverantwortlich getroffen wurde.

\section{Zusammenfassung}

Allgemein anerkannt ist, dass ein früher geäußerter Wunsch in der Betreuung rechtliche Anerkennung erfährt, wenn er ernsthaft, bewusst und frei von Irrtum und Einfluss Dritter gefasst wurde. Der Betroffene muss dazu weder einwilligungs- noch geschäftsfähig sein. Als verbindlicher Wunsch des Betroffenen ist dieser in der Betreuung von dem Betreuer und dem Gericht zu beachten und umzusetzen, soweit er dem aktuellen Wunsch des Betroffenen entspricht. Dies ist nicht gegeben, wenn der Betroffene einzelne antizipiert geäußerte Wünsche oder den gesamten Inhalt seiner Betreuungsverfügung widerruft. Dies gilt, solange der Betroffene einen natürlichen Willen bilden kann. ${ }^{164}$

Die Bindung an den rechtlich anerkannten antizipiert geäußerten Betroffenenwunsch begründet sich in der gesetzlichen Formulierung der $\iint 1897$ Abs. 4, 1901 Abs. 2, 3 und 1901a, des Selbstbestimmungsrechts und der ausdrücklichen Anerkennung dessen durch den $\mathrm{BGH}^{165}$. Ausnahmen von der Bindung an den Betroffenenwunsch sind nur bei Willensmängeln durch fehlende Eigenverantwortlichkeit bei der Wunschbildung anzunehmen bzw. wenn sich der Betroffene bei der Umsetzung solch eines Wunsches zu schädigen droht. Unzutreffend ist daher die Auffassung, dass Vorschläge des Betreuten nur verbindlich seien, wenn sie seinem Wohl nicht zuwiderlaufen. ${ }^{166}$ Nicht auf die Grenze, sondern die grundsätzliche Verbindlichkeit der rechtlich anerkannten, antizipiert geäußerten Wünsche kommt es an.

\section{Reichweite der Betreuungsverfügung}

Die in der Betreuungsverfügung enthaltenen Wünsche sind rechtlich beachtlich. Der Gesetzgeber formulierte in $\int 1901$ Abs. 3 S. 2 das Wohl des Betroffenen und die Zumutbarkeit der Umsetzung des Wunsches durch den Betreuer als Reichweitenbegrenzung der Betroffenenwünsche. Fraglich ist, ob und wenn ja, wie weit diese und andere gesetzliche Regelungen die Reichweite der Betroffenenwünsche bestimmen.

\footnotetext{
164 Berger JZ 2000, 797, 805.

165 Vgl. dazu BGHZ 154, 205, 210.

166 So aber z.B. Rudolf/Bittler $\int 3$ Rn. 2; so ist wohl auch Bienwald zu verstehen (vgl. Bienwald/Sonnenfeld/Hoffmann $\int 1901$ Rn. 25).
} 
1. Wobl des Betroffenen gem. $\int 1901$ Abs. 3 S. 1

a) Problemerörterung

Gem. \1901 Abs. 3 S. 1 hat der Betreuer den Wünschen des Betreuten zu entsprechen, soweit dies dessen Wohl nicht zuwider läuft. Das Wohl des Betreuten bildet nach der gesetzlichen Formulierung eine Grenze für die Reichweite der Bindung an die Betreutenwünsche. \ 1901 Abs. 2 S. 1 gilt als zentrale Norm für die Pflichten des Betreuers und des Betreuungsgerichts. ${ }^{167}$ Danach soll das Wohl des Betroffenen Handlungs- und Entscheidungsmaßstab für alle ihn betreffenden Entscheidungen sein. ${ }^{168}$

Fraglich ist, wie und durch wen das Wohl des Betreuten zu definieren ist. Zu untersuchen ist dabei, ob das Gesetz dem Einzelnen erlaubt, sein Wohl selbst zu definieren. In diesem Fall müsste der Betreuer sich bei der Auslegung der Betroffenenwünsche an diese Definition halten, statt es selber im Sinne des Betroffenen zu bestimmen. Die Gefahr einer Fremdbestimmung in der Betreuung wäre damit stark reduziert.

Eine gesetzliche Legaldefinition vom „Wohl des Betreuten“ fehlt. \ 1901 Abs. 2 gibt eine inhaltliche Konturierung durch S. 2 vor: Das Wohl ist auch subjektiv als Möglichkeit für den Betreuten zu verstehen, sein Leben nach seinen eigenen Wünschen und Vorstellungen zu gestalten. ${ }^{169}$ In bewusster Abkehr zum früher geltenden Vormundschafts- und Pflegschaftsrecht sollte im Betreuungsrecht nach der gesetzgeberischen Idee Wünschen und Vorstellungen des Betroffenen ein hoher Stellenwert zukommen. ${ }^{170}$ \ 1901 soll daher den jeweiligen Fähigkeiten des Betreuten flexibel Rechnung tragen. ${ }^{171}$ Die Vorschrift bestimmt, welche Bedeutung dem Wohl und den Wünschen des Betreuten zukommt, ${ }^{172}$ definiert dies aber nicht ausdrücklich.

Die Materialien zum Betreuungsgesetz behandeln die Frage des Wohls nicht abschließend, aber eingehender. ${ }^{173}$ Statt einer Definition werden Fallgestaltungen aufgelistet. Die Gesetzesbegründung sagt ausdrücklich, dass das Wohl nicht ausschließlich objektiv, sondern aus der Perspektive des Betreuten zu bestimmen und im Rahmen seiner krankheitsbedingt eingeschränkten Fähigkeiten zu gestalten ist. Die Pflicht zur Wunschbeachtung entfällt demnach erst dann, wenn die Gefahr der erheblichen Selbstschädigung besteht. ${ }^{174}$ Ebenso urteilt ein Teil der Literatur, der bei einem Widerspruch von objektivem Wohl und subjektivem Wunsch aufgrund des Selbstbestimmungsrechts des Betreuten im Zweifel annehmen möchte,

\footnotetext{
167 Lipp S. 149; MünchKommBGB/Schwab \ 1901 Rn. 1 ff.; Erman/Roth \ 1901 Rn. 3.

168 MünchKommBGB/Schwab S 1901 Rn. 2.

169 MünchKommBGB/Schwab S 1901 Rn. 2.

170 BtG-DiskE S. 59 ff.

171 Soergel/Zimmermann S 1901 Rn. 2.

172 Soergel/Zimmermann S 1901 Rn. 2.

173 BT-Drucks. 11/4528 S. 133.

174 BT-Drucks. 11/4528 S. 133.
} 
dass der Wunsch dem Wohl des Betroffenen nicht zuwider läuft. ${ }^{175}$ Einigkeit besteht in dem Gedanken, dass dem Betreuten nicht das Recht genommen werden kann, Risiken einzugehen. ${ }^{176}$

Andere versuchen, das Wohl des Betreuten inhaltlich unmittelbar wie das Kindeswohl zu bestimmen. ${ }^{177}$ Dem ist entgegen zu halten, dass das heranwachsende Kind von der Unselbstständigkeit in die Selbstständigkeit geführt werden muss. ${ }^{178}$ Im Fall der Betreuung verhält sich dies gerade konträr: Der Betroffene erhält einen rechtlichen Betreuer, gerade weil er aufgrund einer psychischen Krankheit oder einer körperlichen, geistigen oder seelischen Behinderung seine Angelegenheiten ganz oder teilweise nicht mehr besorgen kann. ${ }^{179}$ Er hat eine umfassende eigenverantwortliche Persönlichkeit, welche er jedoch - zumindest in bestimmten Bereichen - nicht mehr zum Ausdruck bringen kann. Diese Bereiche soll der Betreuer durch seine Tätigkeit kompensieren. Zumeist steigert sich das Bedürfnis der Hilfsbedürftigkeit im Sinne einer Unselbstständigkeit des Betreuten während der Betreuung, statt geringer zu werden, so dass der Betroffene von der Selbstständigkeit in die Unselbstständigkeit begleitet werden muss. ${ }^{180}$ Daher hat das Kindeswohl im Vergleich zum Betreutenwohl ein divergierendes Ziel und kann folglich inhaltlich nicht gleich sein. Bei einer teilweisen Übertragung des Kindeswohls auf das Betreutenwohl soll dem Betreuer einerseits kein Erziehungsrecht gegenüber dem Betreuten zustehen und daher die Notwendigkeit bestehen, das Wohl des Betreuten individuell zu definieren. ${ }^{181}$ Andererseits soll das Wohl funktional bestimmt werden, indem eine Entbindung von der Wunschbefolgungspflicht bei erheblicher Gefährdung von Rechtsgütern wie Leben, Gesundheit und fundamentaler Persönlichkeitsrechte, die dem vom Wunsch verfolgten Interesse des Betreuten entgegenstehen, objektiv definiert wird. ${ }^{182}$ Dabei wird vom Betreuer gefordert, die Krankheit des Betroffenen bei der Entscheidungsfindung zu berücksichtigen und im Zweifel dessen Selbstbestimmungsrecht zu achten. 183 Dafür bleibt bei einer objektiven Definition der Rechtsgüter Leben und Gesundheit kein Raum. Letztlich würde das Wohl des Betroffenen nach der objektiv bestimmbaren Selbstschädigung definiert. Dies entspricht nicht den Zielen des Betreuungsrechts.

\footnotetext{
175 HK-BUR/Bawer \1901 Rn. 52; Knittel \ 1901 Rn. 9; MünchKommBGB/Schwab \ 1901 Rn. 14; Zimmermann beruft sich hingegen auf diesen Grundsatz (Soergel/Zimmermann \ 1901 Rn. 10). 176 BVerfGE 58, 208, 224 ff.; Knittel \$ 1901 Rn. 9.

177 Kollmer 124 ff.; MünchKommBGB/Schwab \ 1901 Rn. 9.

178 Staudinger/Bienwald \1901 Rn. 22; MünchKommBGB/Huber \1626 Rn. 32.

${ }_{179}$ MünchKommBGB/Schwab \$ 1901 Rn. 9.

${ }^{180}$ Staudinger/Bienwald $\int 1901$ Rn. 124a.

181 MünchKommBGB/Schwab \$1901 Rn. 9.

182 Knittel 1901 Rn. 9; MünchKommBGB/Schwab \$ 1901 Rn. 14; Schwab FamRZ 1992, 493, 503; Erman/Holzhauer $\$ 1901$ Rn. 6 ff.

183 MünchKommBGB/Schwab \1901 Rn. 14.
} 
Die Grenze der Wunschbefolgung bei der drohenden Selbstschädigung zu ziehen, also bei Überwiegen des objektiven Interesses des Betroffenen an der Nichterfüllung des Wunsches gegenüber dessen subjektivem Wunsch auf Erfüllung seiner Begehren, ${ }^{184}$ lässt die Kriterien offen, nach denen der Betreuer abzuwägen hat bzw. nach denen die objektiven Interessen des Betreuten im konkreten Fall zu bestimmen sind und ist daher nur bedingt als Maßstab geeignet. 185

b) Verständnis von „Wohl“ in der Betreuung

In der Betreuung wird die Freiheit der Selbstbestimmung für den Bereich, in dem die Betreuung eingerichtet ist, eingeschränkt. Der bestellte Betreuer besorgt die Angelegenheiten des Betreuten mit ihm oder an seiner Stelle und vertritt ihn in seinem Aufgabenkreis gerichtlich und außergerichtlich, vgl. $\ 1901$ Abs. 2 S. 1, $\int$ 1902. Für sein Handeln ist das Wohl des Betreuten Handlungs- und Entscheidungsmaßstab. ${ }^{186}$ Nach $\ 1901$ Abs. 2 S. 2 gehört „Zum Wobl des Betreuten [...] die Möglichkeit, im Rabmen seiner Fäbigkeiten sein Leben nach seinen eigenen Wünschen und Vorstellungen zu gestalten. "Aus der Vorschrift folgt, dass der Betreute sein Leben weitmöglichst selbstbestimmt gestalten soll. ${ }^{187}$ Die Beachtung der Wünsche des Betreuten ist Ausdruck seines Rechts auf Selbstbestimmung in der Betreuung. Der Betreuer hat nach $\$ 1901$ Abs. 3 S. 1 die Wünsche des Betreuten zu achten und auf diese Weise das Selbstbestimmungsrecht des Betroffenen so weit wie möglich $\mathrm{zu}$ wahren. ${ }^{188}$ Die konkrete Entscheidung in der Betreuung ist also keine Entscheidung des Betreuers. Vielmehr hat er diese gemeinsam mit dem Betreuten herbeizuführen, sie zu kontrollieren und ihr nur gegebenenfalls die Anerkennung zu verweigern. ${ }^{189}$ Den Maßstab für diese Entscheidung gibt der Betreute durch seine individuelle Lebensführung, seine Werteinstellungen und Weltansichten vor. Durch diese formuliert er auch in der Betreuung sein Wohl. Dazu bedarf es jedoch keiner ausdrücklichen Äußerung. Vielmehr reicht schlüssiges Verhalten. Als normative Zielvorgabe ${ }^{190}$ ist das Betreutenwohl als „Inbegriff des Integritäts-, Entfaltungs- und Vermögensinteresses des Betreuten gemäß seiner jeweiligen Lebenssituation" "191 zu verstehen.

Der Diabeteskranke bringt durch Verlangen nach 5 Stück Schokolade nach jeder Mahlzeit zum Ausdruck, dass er gerne Schokolade essen möchte. Obwohl dies seiner Erkrankung und damit seinem objektiven Wohl widerspricht, möchte er an

\footnotetext{
184 Kollmer S. 133 f.

${ }^{185}$ Lipp S. 151; Staudinger/Bienwald \ 1901 Rn. 29.

${ }^{186}$ Erman/Holz̧hauer \1901 Rn. 6; MünchKommBGB/Schwab \1901 Rn. 2; nicht so: Staudinger/Bienwald \$ 1901 Rn. 24a.

187 Vgl. nur HK-BUR/Bawer \1901 Rn. 43, 16; grundsätzlich auch Staudinger/Bienwald \ 1901 Rn. 24.

188 MünchKommBGB/Schwab \ 1901 Rn. 14, 16.

${ }^{189}$ Lipp S. $155 \mathrm{ff}$.

${ }_{190}$ Lipp S. 155.

${ }^{191}$ Lipp S. 155, mit weiteren Verweisen in dortiger Fn. 42.
} 
diesem lebenslang gepflegten Genuss festhalten. Das Schokoladeessen stellt somit einen Teil seines Wohls dar. Es ist von dem Betreuer zu beachten, der ihn daran nicht gewaltsam hindern darf, obwohl das Schokoladeessen der objektiven Bewertung widerspricht.

Anders ist die Situation zu beurteilen, wenn seine Wünsche, Werte und Vorstellungen nicht mehr auf einem freien Willen beruhen. Aus konsequenter Anwendung des $\int 1896$ Abs. 1a ergibt sich, dass der Betroffene nur dann sein Wohl bestimmen kann, wenn er nicht einsichtsunfähig ist. ${ }^{192}$ Er darf in seiner Willensbildung also nicht aufgrund einer Krankheit oder Behinderung gestört sein. Dann muss der Betreuer die Definition des Betroffenenwohls ggf. korrigieren. Dabei darf er sich aber nur auf die individuelle, subjektive Situation des Betroffenen stützen. Zu beachten ist dabei immer das Recht des Einzelnen auf die eigene Krankheit. ${ }^{193}$ „Der Betreute, der im Rabmen der Fäbigkeiten des Betreuten sein Leben nach seinen eigenen Wünschen und Vorstellungen gestalten darf, unterliegt dabei keinen anderen Begrenzungen und Einschränkungen als jeder Nichtbetreute ".194

\section{c) Zwischenergebnis}

Die verschiedenen Ansätze zur Definition des Betroffenenwohls führen nicht zu befriedigenden Ergebnissen. Mehrheitlich anerkannt ist, dass der Ansatz ein subjektiver sein muss. Die vorliegende Untersuchung macht deutlich, dass eine Bestimmung des Wohls nur von jedem Einzelnen selbst vorgenommen werden kann. Dies geschieht in Ausübung des Selbstbestimmungsrechts. Der Verfasser einer Betreuungsverfügung formuliert durch die zum Ausdruck gebrachten Wünsche und Vorstellungen konkludent sein Wohl. Diese Wünsche sind gemäß \ 1901 Abs. 3 S. 2 in der Betreuung verbindlich zu beachten. Mit ihnen formuliert der Betroffene sein subjektives Wohl. Die konkrete Bestimmung erfolgt durch Auslegung und ist durch den Betreuer vorzunehmen. Für die Wohlgrenze des \ 1901 Abs. 2 S. 2 bedeutet dies, dass alle Wünsche des Betroffenen in der Betreuungsverfügung verbindlich sind, außer wenn sie nach \1896 Abs. 1a krankheitsbedingt gebildet wurden. Für den Verfasser der Betreuungsverfügung bildet die Wohlgrenze des \ 1901 Abs. 3 somit keine Gefahr der Fremdbestimmung. Vielmehr stellt sie eine Sicherung der selbst bestimmten Wünsche und Vorstellungen dar. Ein im einsichtsfähigen Zustand verfasster Wunsch kann daher dem Betroffenenwohl nicht widersprechen. ${ }^{195}$ Der Wunsch in einer Betreuungsverfügung ist für den Betreuer daher in fast allen Fällen verbindlich.

\footnotetext{
192 Vgl. dazu ausführlicher $\int 2$ C. II. 3.

193 BVerfGE 58, 208, 224 ff.; BVerfG FamRZ 1998, 895, 896; Knittel S 1901 Rn. 9; Münch-

KommBGB/Schwab S 1901 Rn. 14; Staudinger/Bienwald S 1901 Rn. 22.

194 Staudinger/Bienwald \$ 1901 Rn. 28.

195 Bienwald/Sonnenfeld/Hoffmann \ 1901 Rn. 27; HK-BUR/Bauer \ 1901 Rn. 43; BtG-DiskE S. 59.
} 
2. Zumutbarkeit für den Betreuer gem. $\int 1901$ Abs. 3 S. 1

Gem. \1901 Abs. 3 S. 1, 2. HS a.E. hat der Betreuer den Wünschen des Betroffenen nur zu entsprechen, soweit ihre Umsetzung dem Betreuer zuzumuten ist. Fraglich ist, ob die Zumutbarkeit für den Betreuer eine weitere Grenze der Verbindlichkeit der Betroffenenwünsche darstellt.

$\int 1901$ Abs. 3 S. 1 dispensiert den Betreuer bei Unzumutbarkeit von seiner Erfüllungspflicht. ${ }^{196}$ Der Begriff der Unzumutbarkeit ist als unbestimmter Rechtsbegriff ausfüllungsbedürftig. Der Grund der Unzumutbarkeit kann im Ausmaß der geschuldeten Leistung, also der konkreten rechtlichen Betreuung liegen. ${ }^{197}$ Über die Frage der Zumutbarkeit entscheidet der Betreuer. ${ }^{198}$ Die Grenze, die die Unzumutbarkeit eintreten lässt, wurde noch nicht einheitlich abstrakt definiert. Sie kann nicht in der fehlenden Erforderlichkeit der Tätigkeit ${ }^{199}$ oder im Überschreiten des Aufgabenkreises ${ }^{200}$, nicht im Zeitaufwand ${ }^{201}$ und auch nicht in den allgemeinen Rechtsgrenzen ${ }^{202}$ liegen. Fragen der Erforderlichkeit und der Zumutbarkeit sind einzeln und unabhängig von der Zumutbarkeit zu prüfen. Rechtsgrenzen, die die allgemeine Rechtsordnung festsetzt sind von dem Betreuer losgelöst von der Frage der Zumutbarkeit zu beachten. Die erforderliche Zeit muss der Betreuer bei seiner Aufgabenerfüllung aufwenden.

Somit ist die Zumutbarkeit zwar in jedem Einzelfall der Betreuung individuell zu bestimmen, rein faktische, objektiv messbare und begründbare Grenzen können für ihre Beschreibung aber nicht festgelegt werden. Vielmehr lassen die Ausführungen die These zu, dass es keine unzumutbaren Wünsche gibt. Gleichwohl würde sie eine Reichweitenbegrenzung darstellen.

Das österreichische ABGB formuliert keine vergleichbare Reichweitenbegrenzung der Besachwaltetenwünsche. Vereinzelt wird jedoch gefordert, dass die Reichweitenbegrenzung des $\int 1901$ Abs. 3 S. 1 a.E. auch ohne explizite Regelung im österreichischen ABGB gelten müsse. ${ }^{203}$

\footnotetext{
196 Erman/Holzhauer \ 1901 Rn. 17; Staudinger/Bienwald \$ 1901 Rn. 21.

197 Erman/Holz̧auer \$ 1901 Rn. 17; Knittel \$ 1901 Rn. 11; z.B.: Häufigkeit und Dauer des Betreueraufwands (BT-Drucks. 11/4528 S. 134; Soergel/Zimmermann \ 1901 Rn. 12; Münch-

KommBGB/Schwab S 1901 Rn. 18; Knittel S 1901 Rn. 11); eigene Rechte des Betreuers (Jürgens/Jürgens \ 1901 Rn. 11).

198 Knittel S 1901 Rn. 11.

199 Dies meint z.B. Schwab, vgl. MünchKommBGB/Schwab $\int 1901$ Rn. 18.

200 Dies meinen z.B. Knittel \$ 1901 Rn. 8, MünchKommBGB/Schwab \ 1901 Rn. 12 und Staudinger/Bienwald \$ 1901 Rn. 26.

201 BT-Drucks. 11/4528 S. 134; Knittel 1901 Rn. 11; MünchKommBGB/Schwab S 1901 Rn. 18; Palandt/Diederichsen $\int 1901$ Rn. 7.

202 Knittel sieht schon nur solche Äußerungen als Wünsche an, die nicht gegen gesetzliche Verbote verstoßen (Knittel\$ 1901 Rn. 6).

203 Vgl. nur Barth/Ganner dortige Fn. 291.
} 


\section{Gesetzlich zwingende Regelungen}

Wünsche des Betroffenen können nur im Rahmen der allgemeinen gesetzlichen Ordnung wirken, ${ }^{204}$ also nur soweit dies das zwingende Recht erlaubt. ${ }^{205}$ Vorliegend ist fraglich, inwieweit das Betreuungsrecht als das primär maßgebliche Recht für die Einrichtung und Führung der Betreuung zwingende Regelungen formuliert. Der Betroffene kann auf die Betreuung nur einwirken, wenn das Gesetz die konkrete Frage als für den Einzelnen dispositiv ausgestaltet. Nur dann öffnet sich dieser Regelungsbereich der individuellen Einflussnahme des Einzelnen mittels der Betreuungsverfügung. Daher ist im Anschluss unter $\int 3$ bis $\int 6$ zu untersuchen, welche Bereiche des Betreuungsrechts der Disposition des Einzelnen unterliegen können.

\section{Zusammenfassung}

Wünsche und Vorstellungen, die in einer Betreuungsverfügung verfasst sind, sind für die Entscheidungen des Betreuungsgerichts oder des Betreuers verbindlich. Der Grad ihrer Verbindlichkeit ist von Art und Inhalt des Wunsches abhängig, der durch Auslegung zu ermitteln ist. Sein Wohl formuliert der Betroffene in der Betreuungsverfügung in Form seiner Wünsche und Vorstellungen. Sein Wohl stellt in diesem Fall insofern keine Grenze für die rechtliche Geltung seiner Wünsche dar, als sich beides nicht widerspricht. Nur die Zumutbarkeit der Umsetzung der Betreutenwünsche stellt wie die allgemeinen gesetzlichen Grenzen eine Reichweitenbegrenzung der Wünsche einer Betreuungsverfügung dar.

\section{Form der Betreuungsverfügung}

In \ $1901 c^{206}$ spricht das Gesetz über die Betreuungsverfügung als ein Schriftstück. Aus diesem Wortlaut könnte das Schriftformerfordernis abgeleitet werden. Im Rahmen der gesetzlichen Normierung des Rechtsinstituts wurde über die Einführung des testamentarischen Formerfordernisses nach \2231 diskutiert. Dadurch könne dem Verfasser zum einen die Tragweite seines Handelns vor Auge geführt werden, zum anderen gäbe es keine Schwierigkeiten beim Einhalten der Form, wenn der Verfasser im Rahmen seiner Betreuungsverfügung auch seine letztwillige Verfügung abfasse. ${ }^{207}$ Von einem Formerfordernis hat der Gesetzgeber jedoch bewusst abgesehen. ${ }^{208}$ Eindeutige mündliche Aussagen sollen berücksichtigt werden; der Verfasser soll gerade nicht an einer früheren schriftlichen Äuße-

\footnotetext{
204 Staudinger/Bienwald \$ 1901 Rn. 28.

205 Bienwald sieht die Rechte anderer, die verfassungsmäßige Ordnung und das Sittengesetz als Grenze an (Staudinger/Bienwald S 1901 Rn. 22).

206 Durch das 3. BtÄndG wurden zum 01.09.2009 \ 1901a zur Patientenverfügung und \1901b zum Patientenwillen eingefügt. Dadurch wurde \ 1901a (a.F.) zu \ 1901c.

207 Cypionka DNotZ 1991, 571, 586 f.

208 BT-Drucks. 11/4528 S. 128, 208.
} 
rung festgehalten werden, weil der mündliche Widerruf möglich ist. ${ }^{209}$ Die Literatur folgt dieser Regelung weitgehend. ${ }^{210}$ Ein Formerfordernis würde die Gültigkeit bestehender Betreuungsverfügungen in Frage stellen und somit das Selbstbestimmungsrecht des Einzelnen unnötig einschränken. Es birgt den Vorteil der Rechtssicherheit, beschränkt aber den Willensvorrang des Verfassers. ${ }^{211}$ Problematisch ist ein Formzwang zudem, weil die Erklärung des Betroffenen nur deshalb einen geringeren Grad von Verbindlichkeit haben soll, weil sie nicht formgerecht erklärt worden ist. Ist sie auf andere Weise nachgewiesen, besteht jedoch kein Grund, ihr nicht die gleiche Verbindlichkeit zuzubilligen wie einer schriftlich niedergelegten Erklärung. Gerade wenn man dem Selbstbestimmungsrecht des Betroffenen Geltung verschaffen will, dürfen keine Hürden aufgebaut werden, die nicht schon in der Sache selbst begründet sind, wie z.B. das Erfordernis der Einwilligungsfähigkeit. ${ }^{212}$

Aus \1901c lässt sich weder ein Schriftform-, ${ }^{213}$ noch sonst ein Formerfordernis ableiten. Die Betreuungsverfügung ist lediglich der Träger der antizipiert geäußerten, die eigene Betreuung betreffenden Wünsche. Dass diese Wünsche schriftlich abgefasst, notariell beglaubigt oder beurkundet sein müssen, per Videoaufzeichnung oder auf einem sonstigen digitalen Datenträger festzuhalten sind, schreibt das Gesetz nicht vor. Vielmehr kann eine wirksame Betreuungsverfügung in jeglicher Art und Weise verfasst werden.

Aus Beweisgründen empfiehlt sich dennoch, die Betreuungsverfügung schriftlich abzufassen ${ }^{214}$ und ihre Aktualität ggf. sogar regelmäßig durch die erneute Unterschrift zu bestätigen. ${ }^{215}$ Das Betreuungsgericht darf nur auf solche Vorschläge des Betroffenen Rücksicht nehmen, die es als erwiesen erachtet. ${ }^{216}$ Zweifel an der Echtheit eines Vorschlags - also der Echtheit der Äußerung im Rahmen einer Betreuungsverfügung - können mittels einer Beglaubigung ausgeschlossen wer-

${ }^{209}$ BT-Drucks. 11/4528 S. 128, 208.

210 Berger JZ 2000, 797, 804; Birmanns NWB Nr. 52/53 (21.12.1998) S. 4355; Bund JurBüro 2005, 622 f.; Dodegge/Roth C. Rn. 127; Ganner S. 407; Palandt/Diederichsen \1897 Rn. 17, \ 1901 Rn. 5; MünchKommBGB/Schwab \ 1901 Rn. 13 und \ 1897 Rn. 20; Erman/Holz̧hauer \ 1901a Rn. 7; Staudinger/Bienwald S 1901a Rn. 1; Soergel/Zimmermann \1897 Rn. 40, 1901a Rn. 1; Taupitz. A 120; Keilbach FamRZ 2003, 969, 971; Ill-Groß/Sträßner PflR 1999, 126, 128; Epple BWNotZ 1992, 27, 30; als ein Rechtsinstitut, welches zumindest der schriftlichen Form entsprechen muss, sehen dies Becker/Matthias/Hennies/Schirop und Brucker (Becker/Matthias/Hennies/Schirop Intensivmed 1999, 71, 76; Brucker Die BKK 2002, 198, 203).

211 Cypionka DNotZ 1991, 571, 586 f.; Perau MittRhNotK 1996, 285, 290.

212 So urteilt Roth zutreffend in anderem Zusammenhang (Roth JZ 2004, 494, 497).

213 Bienwald/Sonnenfeld/Hoffmann \ 1897 Rn. 57; Dodegge/Roth C. Rn. 127; Epple BWNotZ 1992, 27, 30; Staudinger/Bienwald S 1901a Rn. 1.

214 BT-Drucks. 11/4528 S. 128; Epple BWNotZ 1992, 27, 30; Perau MittRhNotK 1996, 285, 290; Bund JurBüro 2005, 622 f.; Meier BtPrax 2005, 82, 86; Knittel \$ 1901a Rn. 9; HK-BUR/Bauer \ 1901 Rn. 36; Palandt/Diederichsen $\int 1897$ Rn. 17.

215 Schrader SchAZ 2002, 49, 55; Dodegge/Roth C. Rn. 129.

216 BT-Drucks. 11/4528 S. 128; MünchKommBGB/Schwab S 1897 Rn. 20. 
den. ${ }^{217}$ Seit 2005 kann diese Beglaubigung auch durch Urkundspersonen der Betreuungsbehörde vorgenommen werden, vgl. \6 Abs. 2 BtBG. Eine notarielle Beurkundung kann insofern als entbehrlich erachtet werden, als die so beweisfähige Geschäfts- und Einwilligungsfähigkeit des Verfassers keine Voraussetzungen zur Berücksichtigung der Betreuungsverfügung darstellen. ${ }^{218}$ Die Klärung, ob diese vorlag, vermag das Abfassen der Wünsche unter notarieller Beglaubigung zu leisten, weshalb diese sinnvoll sein kann ${ }^{219}$. Dies ist dann empfehlenswert, wenn der Inhalt der Betreuungsverfügung von umfassenden Wünschen auch komplizierter Art geprägt ist oder wenn der Betroffene bereits aufgrund einer Gebrechlichkeit in seiner Gesundheit beeinträchtigt ist.

Leichter zu handhaben ist die einfache Schriftform, weil sie vom Verfasser jederzeit erstellt, geändert und angepasst werden kann. ${ }^{220}$ Sie bürgt - im Vergleich zur mündlichen Äußerung - für eine ausreichende Beweisfunktion.

Eine bestimmte Form ist auch dann nicht nötig, wenn sich die Wünsche auf beurkundungsbedürftige Rechtsgeschäfte beziehen. ${ }^{221}$ Dies ist nur erforderlich, wenn mit der Betreuungsverfügung eine Willenserklärung abgegeben werden soll. 222 In allen anderen Fällen bedarf es der Umsetzung des Wunsches durch den Betreuer, der dann eine eigene Willenserklärung abgibt.

V. Sicherstellen der Wirkung der Betreuungsverfügung

\section{Registrierung im Zentralen Vorsorgeregister}

Das Betreuungsrecht sieht eine Hinterlegungspflicht für Betreuungsverfügungen nicht vor. Einige Bundesländer haben durch jeweilige landesrechtliche Regelungen eine Hinterlegungsmöglichkeit für Betreuungsverfügungen bei Amtsgerichten ermöglicht.223 Einige Länder stellen den Gerichten dabei die Entgegennahme frei, 224 andere verpflichten diese. ${ }^{225}$ Eine bundesweit einheitliche Möglichkeit, die Betreuungsverfügung registrieren zu lassen, besteht seit 2009.226 Das seit 2003 von

217 Langenfeld S. 157; Dodegge/Roth C. Rn. 127.

${ }^{218}$ Langenfeld S. 157 siehe oben \2 C. II. 1. a).

219 Ähnlich Dodegge/Roth C. Rn. 128.

220 Peran MittRhNotK 1996, 285, 291.

221 So aber Epple (Epple BWNotZ 1992, 27, 31).

222 So auch Perau, er fordert nur dann eine Beurkundung, wenn sich aus der Betreuungsverfügung selbst ein Anspruch ergeben soll (Perau MittRhNotK 1996, 285, 290).

223 Übersicht bei HK-BUR/Bauer \$1901a Rn. 25 = Bauer/Klie S. 70 f.

224 So: Baden-Würtenberg, Berlin, Mecklenburg-Vorpommern, Nordrein-Westfalen, vgl. Ausführungen bei HK-BUR/Bauer \$1901a Rn. 25.

225 So: Bayern, Bremen, Hessen, Niedersachsen, Saarland, Sachsen, Sachsen-Anhalt, Thüringen, vgl. Ausführungen bei HK-BUR/Bauer \$1901a Rn. 25.

226 Der vom Kabinett am 20.08.2008 verabschiedete Gesetzentwurf zur Reform des Zugewinnausgleichs- und Vormundschaftsrechts bestimmt die Erweiterung des zentralen Vorsorgeregisters um die separate Erfassung von Betreuungsverfügungen (BT-Drucks. 16/10798; BR-Drucks.: 
der Bundesnotarkammer eingerichtete Zentrale Vorsorgeregister 227 wurde um die separate Erfassung von Betreuungsverfügungen erweitert, vgl. \78a Bundesnotarordnung. Ursprünglich erfasste es nur Vorsorgevollmachten. ${ }^{228}$ Mittlerweile sind im Zentralen Vorsorgeregister ca. 823.765 Vorsorgevollmachten registriert. ${ }^{229}$ Bis zur gesetzlichen Änderung war das Registrieren einer Betreuungsverfügung nur in Kombination mit einer Vorsorgevollmacht möglich. ${ }^{230}$ Eine Anmeldung ist über das Internet oder per Post möglich. Diese umfasst die Aussage, dass und wem eine Vollmacht erteilt wurde. ${ }^{231}$ Im Register sind aufgrund des Datenschutzes nur die allgemeinen Personendaten wie Namen und Anschrift des Vollmachtgebers und des Vollmachtnehmers erfasst. ${ }^{232}$

Mit einer Hinterlegung bei Gericht wird garantiert, dass im Rahmen eines möglichen Verfahrens die Verfügung des Betroffenen dem Gericht vorliegt und Beachtung findet. Dies ist nicht gewährleistet, wenn die Betreuungsverfügung zu Hause - im eigenen Schreibtisch oder dem eines Vertrauten - verwahrt wird und über ihre Existenz niemand informiert wurde. Bei der Einleitung eines Betreuungsverfahrens erfährt das Gericht trotz Ermittlungen von Amts wegen nach \ 26 des Gesetzes über das Verfahren in Familiensachen und in den Angelegenheiten der freiwilligen Gerichtsbarkeit (FamFG) möglicherweise erst verspätet oder überhaupt nicht von der Betreuungsverfügung. Ggf. sind dann Verfahrensteile mit Blick auf die neuen Erkenntnisse zu wiederholen.

Für ein Betreuungsverfahren ist nach \272 FamFG, \ 13 Zivilprozessordnung (ZPO) das Betreuungsgericht des Amtsgerichts örtlich zuständig, in dessen Bezirk der Betroffene seinen gewöhnlichen Aufenthalt hat. ${ }^{233}$ Bei Umzug des Betroffenen muss sich dieser selber darum bemühen, seine Betreuungsverfügung bei dem für den neuen gewöhnlichen Aufenthalt zuständigen Amtsgericht zu hinterlegen, soweit dies möglich ist. Nach momentaner gesetzlicher und tatsächlicher Lage sind die Amtsgerichte miteinander nicht vernetzt. Eine hinterlegte Betreuungsverfügung kann daher nicht auf dem „Amtsweg“ weitergereicht werden. Solange der Betroffene seinen Aufenthalt weiterhin in diesem Gerichtsbezirk hat, behält die

635/08 S. 13 ff., 22 ff.). Das Gesetz zur Änderung des Zugewinnausgleichs- und Vormundschaftsrechts trat zum 01.09.2009 in Kraft.

227 http://www.vorsorgeregister.de/index.php (Stand: Dezember 2009).

228 Möglichkeiten, Vorsorgevollmachten zu hinterlegen gab es aber bereits länger, siehe den Überblick bei Staudinger/Bienwald \$ 1901a Rn. 16.

${ }^{229}$ Stand: 31.12.2008, http://www.elrv.info/_downloads/zvr/ZVR_Jahresbericht_2008.pdf (Stand: Dezember 2009).

230 So auch Bauer/Klie S. 82; entgegen Zimmermann (Rn. 363) ist es nicht nur eine Frage der Überschrift, ob eine Betreuungsverfügung auch in dieses Register aufgenommen werden kann. Lediglich wenn es sich um eine Vorsorgevollmacht kombiniert mit einer Betreuungsverfügung handelt, ist eine Eintragung möglich. Eine davon losgelöste Betreuungsverfügung ist aufgrund ihrer Voraussetzungen und Wirkungen davon jedoch strikt zu trennen.

231 http://www.vorsorgeregister.de/index.php (Stand: Dezember 2009).

232 Die Kritik des Bundesbeauftragten für Datenschutz ist in BT-Drucks. 15/5252 S. 103 enthalten.

233 Zur örtliche Zuständigkeit ausführlich unter $\int 4 \mathrm{E}$. 
Hinterlegung ihre Funktion. Sie gewährleistet, dass der Wunsch des Betroffenen bei Bedarf dem zuständigen Gericht vorliegt. Aufgrund eines Aufenthalts in einem Krankenhaus oder dem Umzug in ein Pflegheim kann der Gerichtsbezirk und somit das zuständige Gericht, in dem der Betroffene seinen gewöhnlichen Aufenthalt hat, wechseln. Wenn der Betroffene dies dem Gericht nicht mitteilt, wird das nun zuständige Gericht von der Verfügung, die dem ehemals zuständigen Gericht vorliegt, keine Kenntnis erhalten. ${ }^{234}$ Eine entsprechende Information kann nun nur von dem Betroffenen oder einem Vertrauten erteilt werden. Der Betroffene ist zur Auskunft häufig gerade nicht mehr in der Lage, der Vertraute muss über die Existenz der Betreuungsverfügung durch den Betroffenen vorab informiert worden sein. Zudem setzt die Auskunft durch die Vertrauensperson voraus, dass der Bekannte über die Einleitung eines Betreuungsverfahrens informiert wurde. Eine solche Informationspflicht ist vom Gesetz nicht vorgesehen. Weil der Kreis der Vertrauenspersonen nicht bekannt ist, kann dies auch tatsächlich wohl kaum durchgeführt werden. Dem Gericht wird in der Kürze der Entscheidungszeit nicht zuzumuten sein, die informierte Vertrauensperson ausfindig zu machen. Eine dadurch entstehende Verfahrensverzögerung erfolgt zu Lasten des Betroffenen und ohne garantierten Erfolg.

Eine Hinterlegung bei den Betreuungsbehörden ist gesetzlich ebenfalls nicht vorgesehen. Diese haben aber nach $₫ 6$ Abs. 1, 2 BtBG einen gesetzlichen Auftrag zur Beratung und Beglaubigung der Betreuungsverfügung. Die Beratungspflicht auf Seiten der Behörden und die zum Teil auf Länderebene gesetzlich festgelegte Hinterlegungsmöglichkeit bei einigen Amtsgerichten, die jedoch untereinander nicht im Austausch stehen, zeigt eine Lücke in der staatlichen Verwaltung der gesetzlich geregelten Betreuung. Die tatsächliche Möglichkeit der Ausübung des Selbstbestimmungsrechts mittels der Betreuungsverfügung findet von der Aufklärung bis zum Gebrauch der Betreuungsverfügung keine garantierte durchgehende Beachtung. Die Subsidiarität der Betreuung als ein wesentliches Ziel des Betreuungsrechts wird durch die Aufklärungspflicht zwar formell befürwortet, jedoch aufgrund fehlender Kooperation in der Praxis nur zum Teil umgesetzt. Zu seinem eigenen Schutz muss der Betroffene momentan einen Hinweis auf seine Betreuungsverfügung bei sich tragen, um mit Sicherheit ihr Beachten zu erzielen. Die Änderung von $\ 78$ a Bundesnotarordnung hat diese Lücke teilweise geschlossen. Danach ist nun auch die separate Registrierung einer Betreuungsverfügung im zentralen Vorsorgeregister möglich.

\section{Ablieferung der Betreunngsverfügung bei Gericht gem. $\int 1901$ c}

Nach $\$ 1901$ c S. 1 hat derjenige, der ,ein Schriftstück besitzt, in dem jemand für den Fall seiner Betreunng Vorschläge zur Auswabl des Betreuers oder Wünsche zur Wabrnehmung der Betreunng geäußert hat, [...] es unverzüglich an das Betreunngsgericht abzuliefern, nachdem er

${ }^{234}$ Knittel S 1901a Rn. 3. 
von der Einleitung des Verfahrens über die Bestellung eines Betreuers Kenntnis erlangt hat". Die Vorschrift formuliert eine Abgabepflicht bei Gericht. ${ }^{235}$ Sinn und Zweck der Norm ist, sicherzustellen, dass eine entsprechende Verfügung auch zur Kenntnis des Gerichts gelangt, ${ }^{236}$ bei dem das Verfahren über die Betreuerbestellung anhängig ist. Dem Wunsch des Betroffenen soll auch dann Geltung verschafft werden können, wenn der Betroffene sich aufgrund von Krankheit oder seiner geistigen Behinderung nicht mehr an das Abfassen oder den Ort der Hinterlegung seiner Betreuungsverfügung erinnern kann. ${ }^{237}$ Die Pflicht gilt ohne ausdrückliche Aufforderung seitens des Gerichts. ${ }^{238}$

Der Gesetzestext nennt in \1901c S. 1 „Schriftstück“. Da eine Betreuungsverfügung in jeder Art und Weise verfasst werden kann, ${ }^{239}$ muss sich die Ablieferungspflicht des \1901c auf jede Form der Betreuungsverfügung erstrecken. Demnach fallen auch Videos u.ä. Träger unter die Ablieferungspflicht nach $\int 1901 c,{ }^{240}$ jegliche offene oder verschlossene ${ }^{241}$ Betreuungsverfügung des Verfassers, unabhängig von Inhalt, Gültigkeit und Material.242 Das Betreuungsgericht kann die Vorlage einer Abschrift verlangen, vgl. 1901c S. 3. Gleiches gilt, wird die Betreuungsverfügung mit einer Vollmacht kombiniert.

Die Pflicht trifft den Besitzer des Originals wie den einer Kopie der Betreuungsverfügung. ${ }^{243}$ Ausgeschlossen ist hier der Verfasser selber, da immer nur sein aktueller Wille maßgeblich ist und er daher entscheiden muss, ob er seine Betreuungsverfügung gelten lassen will. ${ }^{244}$ Die Ablieferungspflicht aus $\ 1901 \mathrm{c}$ besteht nur gegenüber dem Betreuungsgericht ${ }^{245}$ und ist nur von ihm durchsetzbar, ${ }^{246}$ vgl.

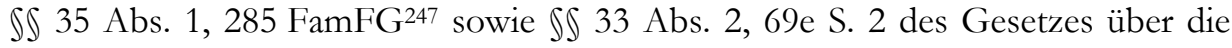

235 HK-BUR/Bauer \$1901a Rn. 47; S. 3 der Vorschrift bezieht sich ausschließlich auf Vorsorgevollmachten.

236 Knittel \1901a Rn. 1; Palandt/Diederichsen \1901a Rn. 1; BT-Drucks. 11/4528 S. 208; MünchKommBGB/Schwab \$1901a Rn. 1; Staudinger/Bienwald \$1901a Rn. 1.

237 Zimmermann Rn. 365.

238 Staudinger/Bienwald S 1901a Rn. 1.

${ }^{239}$ Vgl. \2 C. IV.

240 Zimmermann Rn. 365; für eine analoge Anwendung: MünchKommBGB/Schwab \1901a Rn. 3; anderer Ansicht ist Bienwald (Staudinger/Bienwald \$ 1901a Rn. 9).

${ }^{241}$ Staudinger/Bienwald \$1901a Rn. 8.

242 Perau MittRhNotK 1996, 285, 290, Staudinger/Bienwald \$ 1901a Rn. 8; Zimmermann Rn. 365.

${ }^{243}$ Losgelöst von Eigentumsverhältnissen: HK-BUR/Bauer \$1901a Rn. 49 ff.; Staudinger/Bienwald \1901a Rn. 5 f., 8; MünchKommBGB/Schwab \1901a Rn. 4; Zimmermann Rn. 365.

${ }^{244}$ MünchKommBGB/Schwab \$1901a Rn. 4; im Ergebnis so auch Bienwald, der zwar eine Ablieferungspflicht für den Verfasser anstrebt, aber ausdrücklich eine Selbstbindung des Betroffenen bei aktuell geändertem Willen ausschließt (Staudinger/Bienwald \1901a Rn. 6).

245 Staudinger/Bienwald \$1901a Rn. 13.

246 Palandt/Diederichsen \$ 1901a Rn. 1 (Zwangsgeld oder unmittelbarer Zwang).

$247 \int 285$ FamFG mit der nichtamtlichen Überschrift „Herausgabe einer Betreunngsverfügung oder der Abschrift einer Vorsorgevollmacht" bestimmt: „In den Fällen des $\int 1901$ a des BGBs erfolgt die Anordnung der Ablieferung oder Vorlage der dort genannten Schriftstïcke durch Beschluss. "\$ 69e Abs. 1 S. 2 FGG bestimmte: „Das Vormundschaftsgericht kann im Fall des $\int 1901$ a des BGBs den Besitzer einer Betreuungsverfügung durch Festsetzug von Zwangsgeld zur Ablieferung der Betreunngsverfügung anbalten. "Nach der Ge- 
Angelegenheiten der freiwilligen Gerichtsbarkeit (FGG) ${ }^{248}$. Sie begründet keinen durch den Verfasser durchsetzbaren Anspruch auf Herausgabe gegen den Besitzer, diesbezüglich gelten die allgemeinen schuld- und sachenrechtlichen Ansprüche. ${ }^{249}$ Wenn Grund zur Annahme besteht, dass jemand ein ablieferungspflichtiges Schriftstück besitzt, kann dieser vom Gericht zur Ablieferung oder Vorlage angehalten werden, notfalls sogar durch Festsetzung von Zwangsgeld oder Anordnung von Zwangshaft, vgl. \$S 35, 285 FamFG $^{250}$.

Berufsgruppen - wie Notare -, die durch die Pflicht des $\int 1901$ c in eine Kollision mit der beruflichen Schweigepflicht kommen, werden mit Entstehen der Ablieferungspflicht von ihrer Schweigepflicht durch den Betroffenen zumindest konkludent entbunden sein. Andernfalls kämen die Wünsche des Betroffenen im Rahmen des Betreuungsverfahrens nicht zum Tragen, womit die Betreuungsverfügung bedeutungslos werden würde. ${ }^{251}$

S 1901c S. 1 legt die Pflicht dem Verwahrer auf, „nachdem er von der Einleitung eines Verfahrens über die Bestellung eines Betreuers Kenntnis erlangt hat. "252 Im Gesetzgebungsverfahren befürwortete der Bundesrat den Eintritt der Betreuungsbedürftigkeit. ${ }^{253}$ Bienwald fordert eine jederzeitige Abgabemöglichkeit. ${ }^{254}$ Das Betreuungsgericht dürfe eine Betreuungsverfügung grundsätzlich nicht zurückweisen, sondern müsse sie verwahren. Gegen eine solche Möglichkeit ohne eine zentrale Erfassung der Verfügung spricht bereits das Problem der Zuständigkeit des das Verfahren einleitenden Gerichts. \78a Bundesnotarordnung, nach der Betreuungsverfügungen mittlerweile auch einzeln im Zentralen Vorsorgeregister registriert werden können, unterstützt Bienwalds Forderung.

setzesbegründung soll die Regelung \ 285 FamFG der Regelung \69e Abs. 1 S. 2 FGG entsprechen. Dafür spricht auch die Überschrift, die von Betreuungsverfügung spricht, dem man Beachtung schenken kann, auch wenn die nicht amtlich ist. So dass davon auszugehen ist, dass es sich bei der Verweisung auf $\$ 1901$ a um einen Redaktionsfehler handelt.

248 Durch das FamFG wurde das familiengerichtliche Verfahren grundlegend neu geregelt. Das FamFG trat am 01.09.2009 in Kraft und tritt unter anderem an Stelle des FGG. Viele Regelungen des FGG wurden inhaltlich gleich lautend in das FamFG übernommen. Probleme, die bereits in Bezug auf die Regelungen des FGG diskutiert wurden, haben daher Bestand. Daher werden an den entscheidenden Stellen neben den neuen Regelungen des FamFG die bis zum 31.08.2008 geltenden $\$ \int$ des FGG genannt.

249 Staudinger/Bienwald \$1901a Rn. 17.

250 \ 69e Abs. 1 S. 3 i.V.m. \ 83 Abs. 2 FGG, \ 901 ZPO.

251 Etwas anders sieht dies Bauer, der sich v.a. auf das Argument stützt, dass der Betroffene eine Eintragung im Vorsorgeregister nicht wünschte und so - wenn auch nach Einzelfallentscheidung - im Zweifel die Schweigepflicht des Notars bejaht und \$ 1901a (a.F.) leer laufen lassen will (HK-BUR/Bauer \$1901a Rn. 50).

252 So auch die heute herrschende Meinung, vgl. KG Berlin FamRZ 1995, 1295, 1295 f.; Peran MittRhNotK 1996, 285, 290 und Bund RNotZ 2004, 23, 24; Palandt/Diederichsen \1901a Rn. 1; MünchKommBGB/Schwab \$1901a Rn. 5; KG FamRZ 1995, 1295, 1296; Bauer/Klie S. 76; HKBUR/Bauer \1901a Rn. 52; Staudinger/Bienwald \1901a Rn. 10; Zimmermann Rn. 365.

253 Vorschlag des Bundesrates auf den Regierungsentwurf des BtG hin, der \1901a nicht vorsah; BT-Drucks. 11/4528 S. 208.

254 Bienwald BtG, 3. Aufl. \1901a Rn. 10. 
Eine Ablieferungspflicht mit Eintritt der Betreuungsbedürftigkeit ließe für den Verwahrer die Verpflichtung entstehen, dem Gericht die Betreuungsbedürftigkeit des Betroffenen anzuzeigen. Diese Pflicht einer Privatperson kann aus dem Betreuungsrecht heraus nicht begründet werden. ${ }^{255}$ Sie würde zudem das Verhältnis zwischen Verwahrer und Betroffenem unnötig belasten. ${ }^{256}$ Nach einhelliger Meinung erlischt die Pflicht mit Aufhebung der Betreuung. ${ }^{257}$

\section{Beratung beim Abfassen der Betreunnsverfügung}

Die Betreuungsverfügung kann jeder Einzelne ohne fachliche Beratung abfassen. Eine Beratungspflicht als Wirksamkeitsvoraussetzung normiert das Gesetz nicht. Fraglich ist, ob durch die für die Behörden und Vereine gesetzlich vorgesehene Beratungsmöglichkeit eine Beratungspflicht besteht, wenn der Einzelne diese einfordert.

Durch eine umfassende Beratung über die individuelle Beratung soll der Betroffene zum einen über die verschiedenen Vorsorgemöglichkeiten informiert und in die Lage der Entscheidungsfähigkeit versetzt werden, zum anderen kann sie dem späteren Adressaten das Verständnis für die Inhalte erleichtern. ${ }^{258}$ Letzteres jedoch im Zweifel nur, wenn dieser in die Beratung einbezogen wurde und daher den Hintergrund der formulierten Inhalte kennt.

Funktional kämen zur Beratung Notare, Anwälte, Gerichte, Betreuungsbehörden und Betreuungsvereine in Betracht. Für den Bürger interessant wäre eine amtliche, kostenlose Beratung.

Eine Beratungspflicht könnte sich für die Betreuungsbehörde aus $\int 6$ Abs. 1 S. 2 BtBG ergeben. Danach ,fördert sie die Aufklärung und Beratung über Vollmachten und Betreuungsverfügungen. "Fraglich ist die Bedeutung der Förderung von Aufklärung und Beratung. Wenn damit eine umfassende Rechtsberatung gemeint ist, bestünde de lege lata eine behördliche Beratungspflicht. Dies würde die Behör$\mathrm{de}^{259} \mathrm{zu}$ umfassenden, vollständigen, sorgfältigen und individuellen Beratungen verpflichten, deren Verletzung zu Schadensersatz verpflichten würde.

Unter Fördern der Behörde verstehen einige die rein finanzielle Unterstützung, ${ }^{260}$ andere das Behandeln des Themas in der Öffentlichkeit. ${ }^{261}$ Auch die Be-

\footnotetext{
255 Gegenäußerung der Bundesregierung BT-Drucks. 11/4528 S. 227.

256 Gegenäußerung der Bundesregierung in BT-Drucks. 11/4528 S. 227; HK-BUR/Bauer \$1901a Rn. 55.

257 Palandt/Diederichsen \1901a Rn. 1; HK-BUR/Bawer \1901a Rn. 56; MünchKommBGB/Schwab \1901 Rn. 5; Staudinger/Bienwald \$1901a Rn. 12.

${ }^{258}$ Lipp Patientenautonomie S. 29.

${ }^{259}$ Die Behörde wird mittlerweile allgemein als Adressat angesehen, vgl. Bienwald/Sonnenfeld/Hoffmann $₫ 6$ BtBG Rn. 1.

${ }^{260}$ Dodegge/Roth J. Rn. 49a; nach Knittel muss es sich dabei nicht zwingend um finanzielle Förderung handeln, sondern auch um das Vorantreiben des Themas in der Öffentlichkeit, vgl. \$ 6 BtBG Rn. 22; kritisch: Bienwald/Sonnenfeld/Hoffmann $\int 6$ BtBG Rn. 2.
} 
deutung von Aufklärung und Beratung wird unterschiedlich verstanden. Nach $₫ 1$ Abs. 1 S. 1 RDG $^{262}$ ist unter Rechtsberatung die Unterrichtung des Ratsuchenden über die Rechtslage eines Einzelfalles sowie die zu ergreifenden Maßnahmen und die Hilfeleistung bei der Sammlung von Unterlagen zu verstehen. ${ }^{263}$ Rechtsberatung liegt demnach nur dann vor, wenn sich die Beratung - vom Standpunkt des Ratsuchenden aus - auf einen ganz bestimmten Einzelfall bezieht. ${ }^{264}$ Dies ist insbesondere dann gegeben, wenn es um die Aufklärung einer bestimmten Rechtslage des praktischen Lebens geht ${ }^{265}$ oder über die Möglichkeiten, Folgerungen aus der Rechtslage zu ziehen bzw. den Rat, sich in einem bestimmten Sinne zu verhalten. ${ }^{266}$ Das RBerG war in dieser Frage restriktiv zu verstehen. ${ }^{267}$ Dies sollte auch für das RDL gelten. Fraglich ist, ob $\int 6$ Abs. 1 S. 2 BtBG die Behandlung eines Einzelfalles meint. S. 1 spricht von der Tätigkeit einzelner Personen. Dies ist darauf ausgerichtet, dass die Behörde einzelne Personen bei ihrer Tätigkeit zugunsten Betreuungsbedürftiger unterstützen soll. S. 2 ist anders aufgebaut. Dabei geht es nicht um die Unterstützung bei einer Tätigkeit, sondern um die Verbreitung der Rechtsinstitute Vorsorgevollmacht und Betreuungsverfügung. ${ }^{268}$ Dies steht im Zusammenhang mit dem allgemeinen Bemühen des Gesetzgebers im Rahmen des 2. BtÄndG, die rechtliche Betreuung zu vermeiden und zu diesem Zweck Alternativen aufzuzeigen. Somit geht es um die allgemeine Verbreitung und nicht um den Einzelfall. Bereits dadurch unterscheidet sich die Beratung des $\int 6$ Abs. 1 S. 2 BtBG von der Rechtsberatung i.S.d. RDL. Zudem sind Behörden dazu angehalten, bei komplexen und schwierigen Rechtsfragen, die Betroffenen an einen Rechtsanwalt und Notar zu verweisen. ${ }^{269}$ Nach Art. 1 \ 3 Nr. 1 RDG fällt „Rechtsberatung, [...] die von Behörden [...] im Rahmen ibrer Zuständigkeit ausgeübt wird" nicht unter den Erlaubnisvorbehalt des $\int 1$ Abs. 1 RDL. Somit könnten Mitarbeiter der

\footnotetext{
${ }^{261}$ Im Sinne von Vorantreiben durch Verbreitung entsprechender Broschüren und Informationsblättern, vgl. HK-BUR/Walther $\$ 6$ BtBG Rn. 27; so im Ansatz auch Knittel \6 BtBG Rn. 22; Jurgeleit/Kania/Langholf/Schmidt \$ 6 BtBG Rn. 8.

262 Das RBerG wurde am 01.07.2008 durch das RDG ersetzt.

${ }^{263}$ Chemnitz/Johnigk Art. 1 \& 1 RBerG Rn. 36.

${ }^{264}$ Chemnitz/Johnigk Art. 1 \& 1 RBerG Rn. 37.

265 Heinken S. 3; Chemnitz/Johnigk Art. 1 \1 RBerG Rn. 36; HK-BUR/Walther \$ 6 BtBG Rn. 27.

${ }^{266}$ Chemnitz/Johnigk Art. 1 \1 RBerG Rn. 36; Heinken S. 3.

267 BGH NJW 1956, 591; BGH NJW 1981, 1616.

268 Knittel \ 6 BtBG Rn. 23; ähnlich: Fröschle/Kubrke \ 6 BtBG Rn. 11; Zimmermann Rn. 363.

${ }^{269}$ Zweck des RBerG war zum einen, der Schutz des Verbrauchers vor unsachkundigen Rat (Bauer/Klie E. 2.; Rennen/Caliebe Art. 1 \& 1 RBerG Rn. 9; BVerfGE 41, 378, 390, 393; BVerfGE 97, 12, 26), zum anderen aber auch die Gewährleistung einer reibungslosen Rechtspflege (Rennen/Caliebe Art. 1 \1 Rn. 9 RBerG) und den Schutz derer, die dem strengen Berufsrecht unterworfen sind, vor unqualifizierter und damit zumeist billigerer Konkurrenz (BGH NJW 1962, 2010; BGH NJW 1967, 1558). Daher wird die Behörde nicht unmittelbar aus Satz 2 zur Tätigkeit verpflichtet. Vielmehr soll sie die Aufklärungs- und Beratungstätigkeit anderer fördern, also mittelbar aktiv sein (Bienwald/Sonnenfeld/Hoffmann \6 BtBG Rn. 1); anders hingegen Jürgens/Winterstein \6 BtBG Rn. 9, der in Satz 2 eine Verpflichtung der Behörden zur Beratung sieht.
} 
Betreuungsbehörden von dem Erlaubnisvorbehalt zur Rechtsberatung befreit sein. Jedoch begründet das BtÄndG von 1999 gerade keine Zuständigkeit der Betreuungsbehörden für eine rechtliche Einzelfallberatung. ${ }^{270}$ Dem Wortlaut nach geht die Aufgabe der Behörde aus $\int 6$ Abs. 1 S. 2 BtBG nicht über die allgemeine Information ohne konkreten Einzelfallbezug hinaus. ${ }^{271}$ Eine Beratung im Sinne des RBerG ist also in $\int 6$ Abs. 1 S. 2 BtBG nicht vorgesehen.

Eine Beratungspflicht für Betreuungsvereine ergibt sich auch nicht aus $\int 1908 \mathrm{f}$ Abs. 1 Nr. 2 a. Danach kann ein ,rechtsfähiger Verein [...] als Betreuungsverein anerkannt werden, wenn er gewährleistet, dass er planmäßig über Vorsorgevollmachten und Betreuungsverfügungen informiert". Der Gesetzgeber wollte Betreuungsvereinen ausdrücklich die Pflicht zur allgemeinen Information auferlegen, zugleich aber auch durch \1908f Abs. 4 die Möglichkeit schaffen, Ratsuchende individuell zu beraten. ${ }^{272}$ Abs. 4 stellt dabei lediglich auf das Rechtsinstitut Vorsorgevollmacht ab. Ein Hinweis auf die Betreuungsverfügung fehlt. Dies ist Folge der gesetzgeberischen Bestrebung, rechtliche Betreuungen zu vermeiden. Mittels des Rechtsinstituts Betreuungsverfügung lässt sich dies nicht erreichen. Damit ist $\int 1908 \mathrm{f}$ Abs. 1 Nr. 2 a nur eine Aufgabenzuweisung zur allgemeinen planmäßigen Information über die Betreuungsverfügung. Eine Kollision durch \ 1908 f Abs. 4 mit Art. 1 \& 3 RDG besteht insoweit nicht, als die Regelung des $\int 1908 \mathrm{f}$ vorgeht. ${ }^{273}$ Somit besteht lediglich die Beratungsmöglichkeit durch Betreuungsvereine, eine individuelle Beratungspflicht besteht nicht. Dies erscheint i.E. nicht sinnvoll. Zu einer allgemein Informationspflicht muss die Aufklärung über die Betreuungsverfügung gehören. Andernfalls fehlt dem Ratsuchenden u.a. die Abgrenzungs- und damit die Entscheidungsmöglichkeit.

\section{Zusammenfassung}

Nach \1901 Abs. 2 S. 2 und Abs. 3 S. 2 sind Inhalt der Betreuungsverfügung die antizipiert geäußerten Wünsche und Vorstellungen des Betroffenen in Bezug auf seine eigene Betreuung. Die Erklärungen des Betroffenen sind für Betreuer und Gericht verbindlich, soweit es sich tatsächlich um seinen, und nicht den Willen eines Dritten handelt, er bei der Willensbildung nicht einem Irrtum unterlag und diesen bewusst und ernsthaft verfasst hat. Nicht an die Betreuungsverfügung sind

\footnotetext{
270 Bauer/Klie E. 3.; BT-Drucks. 15/2494 S. 31; Fröschle/Kubrke \6 BtBG Rn. 11; Knittel \$ 6 BtGB Rn. $23-25$.

${ }^{271}$ HK-BUR/Walther \ 6 BtGB Rn. 29; Fröschle/Fröscble \6 BtBG Rn. 11; Bienwald/Sonnenfeld/Hoffmann $₫ 6$ BtBG Rn. 11; Bauer/Klie S. 162; Sonnenfeld FamRZ 2005, 941, 944; Dodegge/Roth J. Rn. 49a; Jurgeleit/Kania/Langholf/Schmidt \$ 6 BtBG Rn. 22; anderer Ansicht - jedoch ohne Begründung - ist Winterstein, wonach die Behörde mehr als reine Information zu leisten habe (Jürgens/Winterstein $\$ 6$ BtBG Rn. 9).

272 Vgl. BT-Drucks. 15/2494 S. 31; so auch: HK-BUR/Walther $₫ 6$ BtGB Rn. 31; Bienwald BtPrax 1998, 164 dort Fn. 2; Bauer/Klie S. 160; Dodegge/Roth J. Rn. 49a.

273 BT-Drucks. 15/2494 S. 31.
} 
Anforderungen zu stellen, sondern an die darin enthaltenen Wünsche. Durch das Formulieren dieser definiert der Betroffene sein individuelles, subjektives Wohl und somit die Reichweite seiner Wünsche. Beides ist dann nicht mehr möglich, wenn der Betroffene nicht zur freien Willensbildung in der Lage, also einsichtsunfähig i.S.v. \ 1896 Abs. 1a ist bzw. sich aufgrund fehlender Eigenverantwortlichkeit durch seinen Wunsch selbst zu schädigen droht. Eine Reichweitenbeschränkung der Wünsche durch das Wohl nach \ 1901 Abs. 3 S. 1 ist außer in diesen zwei Ausnahmen nicht möglich. Diese ist in der Zumutbarkeit der Wunschumsetzung für den Betreuer, den zwingenden Regelungen des Betreuungsrechts und der allgemeinen Rechtsordnung zu sehen.

Welchen genauen Inhalt eine Betreuungsverfügung hat, bestimmt ihr Verfasser. Er legt fest, welchen Gehalt er seinen Äußerungen im Rahmen der Betreuungsverfügung zukommen lassen will. Gibt er einen eindeutigen Wunsch ab, dann sind Betreuer und Gericht strikt an diese konkreten Vorgaben gebunden. Stellt der Betroffene hingegen Wertvorstellung oder Ansichten dar, muss der Betreuer selbst die entsprechenden Entscheidungen unter Berücksichtigung und in Orientierung an den genannten Wertvorstellungen und Auffassungen des Betroffenen treffen. ${ }^{274}$

Wirksamkeitsvoraussetzungen für das Rechtsinstitut Betreuungsverfügung normiert das Gesetz nicht. Nach \1901a S. 1 formuliert es nur die Pflicht des Betreuungsverfügungsbesitzers, diese unverzüglich nach Bekanntwerden der Einleitung eines Betreuungsverfahrens bei Gericht abzuliefern. 



\section{\3 Einfluss der Betreuungsverfügung auf die Auswahl des Betreuers}

Einen Katalog mit ausdrücklichen Anforderungen für die Betreuerauswahl enthält das Gesetz in $\$ 1897$. Weitere Kriterien sind in $\int \$ 1898-1900$ geregelt und ergeben sich im Übrigen aus einer Gesamtschau des Betreuungsrechts. Die Auswahl des Betreuers ist rechtlich keine eigenständige Einzelentscheidung, sondern nach $§ 1896$ Bestandteil der einheitlichen Entscheidung der Betreuerbestellung. ${ }^{275} \mathrm{Gem}$. \1897 Abs. 4 und 5, \1901c S. 1 und 2 kann jeder Volljährige in einer Betreuungsverfügung Vorschläge, Wünsche und Vorstellungen in Bezug auf einen künftig möglicherweise notwendigen Betreuer äußern. Einem solchen Vorschlag ist gem. $\$ 1897$ Abs. 4 S. 1 zu entsprechen. Der derart Benannte wird im Folgenden als sog. Wunschbetreuer bezeichnet. Fraglich ist, in welchem Umfang der Betroffene mittels seiner Wünsche und Vorschläge auf die Auswahl seines Betreuers einwirken kann. Zu untersuchen ist dabei, wann das Gesetz die Einflussnahme des Einzelnen vorsieht und erlaubt, das Gesetz also dispositiv ist. Im Übrigen ist zwischen dem „Ob“ und „Was“ der Betroffenenwünsche zu unterscheiden. Ob die Wünsche des Betroffenen ,erfüllt“" werden können, ist eine Frage der gesetzlichen Vorgaben; was der Betroffene erreichen will, eine Frage der Auslegung.

\footnotetext{
275 BT-Drucks. 11/ 4528 S. 124: Der Richter hat anhand der genannten Vorgaben die maßgeblichen Kriterien zu ermitteln. Vgl. auch Staudinger/Bienwald \$ 1897 Rn. 10.
} 


\section{A. Auswahl des Betreuers gem. $\int \mathbb{S} 1897 \mathrm{ff}$.}

Fraglich ist, inwieweit die $\iint 1896$ ff. zwingende Regeln für die Auswahl und Benennung des Betreuers vorgeben bzw. einen Freiraum für individuelle Betroffenenwünsche gewähren. Dabei ist v.a. zu untersuchen, was der Betroffene mit seinen Wünschen erreichen will. Dies zu ermitteln, ist eine Frage der Auslegung.

I. $\quad$ Vorschlag gem. $\int 1897$ Abs. 4 S. 1

\section{Einzelperson, Personenkreis oder-gruppe}

Hat die nun zu betreuende Person einen Betreuer vorgeschlagen, ist dessen Vorschlag gem. \1897 Abs. 4 ,zu entsprechen, wenn es dem Wobl des Volljährigen nicht zuwider läuft". Ist diese Voraussetzung erfüllt, hat das Betreuungsgericht dem Vorschlag des Betreuten ohne Einschränkung zu folgen und diese vorgeschlagene Person als Betreuer zu bestellen. ${ }^{276}$ Dem Richter steht bei einem eindeutigen Vorschlag mithin kein Ermessen zu. ${ }^{277}$ Dies gilt selbst dann, wenn objektiv eine andere Person besser zur Betreuung des Betroffenen geeignet ist. ${ }^{278}$ Die Grenze ist nach $\int 1897$ Abs. 4 dann erreicht, wenn die Benennung des Wunschbetreuers dem Wohl des Betreuten zuwider läuft. Nach der herrschenden Meinung ist dies gegeben, wenn die konkrete Gefahr besteht, dass der Wunschbetreuer die rechtliche Betreuung nicht zum Wohl des Betroffenen führen kann oder will. ${ }^{279}$

Aus der Wunschbeachtungspflicht des $\int 1901$ Abs. 3 S. 1 ist abzuleiten, dass der Wunschbetreuer immer dann zum Betreuer bestellt werden muss, wenn dieser Wunsch nicht im Zustand der Einsichtsunfähigkeit gem. \1896 Abs. 1a verfasst wurde bzw. seine Umsetzung nicht zur Selbstschädigung des Betroffenen führt.

276 BT-Drucks. 11/4528 S. 127; Bienwald/Sonnenfeld/Hoffmann \1897 Rn. 53, 58; MünchKommBGB/Schwab \1897 Rn. 19, 34; Palandt/Diedericbsen \1897 Rn. 20; Bamberger/Roth/Müller \1897 Rn. 14; Erman/Holzhauer \$1897 Rn. 3; Knittel \$ 1897 Rn. 17; Staudinger/Bienwald \ 1897 Rn. 28; Perau MittRhNotK 1996, 285, 286; BayObLG FamRZ 1997, 1360, 1361; BayObLG FamRZ 2004, 976, 977; OLG Düsseldorf FamRZ 1998, 510; KG FamRZ 1995, 1442, 1443.

277 Perau MittRhNotK 1996, 285, 286; Schwab FamRZ 1992, 493, 501; Bienwald/Sonnenfeld/Hoffmann \1897 Rn. 56; Staudinger/Bienwald \1897 Rn. 28; BayObLG FamRZ 2004, 976, 977.

278 Perau MittRhNotK 1996, 285, 286; MünchKommBGB/Schwab \ 1897 Rn. 23; Staudinger/Bienwald \$ 1897 Rn. 28; Schwab FamRZ 1992, 493, 501 f.; Knittel \$1897 Rn. 17a; Palandt/Diederichsen \1897 Rn. 20; HK-BUR/Bauer 1897 Rn: 72; Palandt/Diederichsen \1897 Rn. 20; OLG Düsseldorf FamRZ 1996, 1373, 1373 f.; BayObLG FamRZ 1999, 53; OLG Köln FamRZ 1999, 811.

279 Bienwald/Sonnenfeld/Hoffmann \1897 Rn. 58; Erman/Hoľ̨hauer 1897 Rn. 3; MünchKommBGB/Schwab \1897 Rn. 23; Schwab FamRZ 1990, 681, 685; Staudinger/Bienwald \$ 1897 Rn. 28; Knittel \$1897 Rn. 17; Palandt/Diederichsen \1897 Rn. 20; BT-Drucks. 11/6949 S. 71; BayObLG FamRZ 1996, 1373; BayObLG FamRZ 1997, 1360, 1361; BayObLG BtPrax 2002, 36, 37; BayObLG FamRZ 2004, 976, 977; OLG Düsseldorf FamRZ 1996, 1373, 1374; OLG Brandenburg FamRZ 2001, 936, 937. 
Die in $\int 1897$ Abs. 4 formulierte Regelung gilt auch dann, wenn der Betroffene mehrere Personen als Wunschbetreuer in der Betreuungsverfügung benennt. Über die Verbindlichkeit des Vorschlags mehrerer Wunschbetreuer wird gestritten. Dabei ist zu analysieren, was der Betroffene durch die Nennung Mehrerer zum Ausdruck bringen möchte. Es sind verschiedene Konstellationen zu unterscheiden.

Wünscht der Betroffene für verschiedene Aufgabenkreise verschiedene Betreuer, benennt er zwar mehrere Wunschbetreuer, genau genommen handelt es sich aber um die Benennung eines bestimmten Wunschbetreuers für eine bestimmte Angelegenheit bzw. einen bestimmten Aufgabenkreis. In diesem Fall ist der Vorschlag des Betroffenen also eindeutig und zuzuordnen. Daher muss dann die für den jeweiligen Aufgabenkreis vorgeschlagene Person nach \1897 Abs. 4 S. 1 durch das Gericht bestellt werden. ${ }^{280}$ Diese Auslegung unterstützt \ 1899 Abs. 1 S. 1 und S. 2. Danach kann das Gericht dann mehrerer Betreuer bestellen, wenn die Angelegenheiten des Betreuten dadurch besser besorgt werden können. Nach dem Wortlaut hat das Gericht in dieser Frage einen Ermessensspielraum. Fraglich ist, wann das Gericht den Ermessensspielraum ausüben darf. Legt man die Regelung so aus, dass das Gericht nur dann mehrere Betreuer bestellen darf, wenn es glaubt, dass dadurch die Besorgung der Angelegenheiten besser erfolgt, dann stünde sie der Benennungsmöglichkeit nach \ 1897 Abs. 4 S. 1 entgegen. Eine solche Einschränkung wurde in der Gesetzesbegründung nicht niedergelegt. Die Systematik unterstützt eine andere Auslegung. Dass nämlich eine Ernennung Mehrerer gerade dann möglich ist, wenn das Gericht von der besseren Besorgung der Angelegenheiten ausgeht. Dann steht \ 1899 auch nicht im Widerspruch zu $\int 1897$ Abs. 4 S. 1.281

Anders ist der Fall zu bewerten, in dem der Betroffene durch die Benennung mehrerer Wunschbetreuer zum Ausdruck bringen will, dass er die gleichzeitige Bestellung aller - für alle erforderlichen Angelegenheiten - wünscht. Gemäß $\int 1899$ Abs. 3 können mehrere Betreuer mit demselben Aufgabenkreis die Angelegenheiten des Betreuten nur gemeinsam besorgen, soweit das Gericht nichts anderes bestimmt und mit dem Aufschub keine Gefahr verbunden ist. Das Gesetz sieht die Möglichkeit der gleichzeitigen Betreuung durch mehrere Betreuer im gleichen Aufgabenkreis also vor. Da nach obiger Auslegung \1899 in entsprechender Anwendung des $\int 1896$ Abs. 4 nicht als zwingende Regelung zu verstehen ist, kann der Betroffenen durch die Benennung mehrer Wunschbetreuer auf die Bestellung dieser für dieselbe Angelegenheit hinwirken. ${ }^{282}$

\footnotetext{
280 Dodegge/Roth C. Rn. 139; Perau MittRhNotK 1996, 285, 287.

${ }^{281}$ Für diese Auslegung spricht auch das System der Vergütung und der Aufwendungsentschädigung

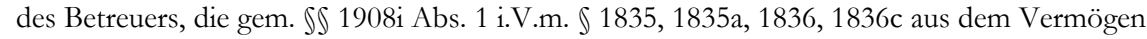
des Betreuten zu erfolgen hat.

282 Anders sieht dies Bienwald (Staudinger/Bienwald \ 1899 Rn. 5).
} 
Wünscht der Betroffene die Ernennung einer Person aus einem konkret umschriebenen Personenkreis, nennt er dem Betreuungsgericht damit Alternativen. ${ }^{283}$ Nennt der Betroffene einen bestimmten Kreis von Personen, sind diese durch das Betreuungsgericht individuell erkennbar. Bei diesem Vorschlag kann der Betroffene sich jeden aus dem vorgeschlagenen Kreis als seinen Betreuer vorstellen. ${ }^{284}$ Hier bleibt dem Betreuungsgericht ein Auswahlermessen - beschränkt auf den genannten Personenkreis. ${ }^{285}$ Aus diesem muss das Betreuungsgericht dann den für den erforderlichen Aufgabenkreis geeignetesten Betreuer auswählen. ${ }^{286}$ Es kann eine dieser vorgeschlagenen Personen als Betreuer für sämtliche Aufgabenbereiche bestellen oder aber für mehrere Aufgabenbereiche je einen anderen Betreuer aus dem vorgeschlagenen Kreis auswählen. ${ }^{287}$ Die Nennung einer durch bestimmte Personen gebildete Gruppe ist rechtlich nicht anders zu bewerten, als die Benennung einer einzelnen Person, ${ }^{288}$ weil die Benennung jedes einzelnen Personenkreismitgliedes dem Wunsch des Betroffenen entspricht. Daher ist für die Frage der Verbindlichkeit des Vorschlags derselbe Maßstab wie in $\$ 1897$ Abs. 4 S. 1 anzulegen, so dass ein derartig konkret formulierter Wunsch verbindlich ist. ${ }^{289}$

Im Zusammenhang mit der Nennung eines Vereins ${ }^{290}$ als Gruppe stellt sich die Problematik des gesetzlichen Vorrangs der Einzelbetreuung, die nicht zur Disposition des Betroffenen steht ${ }^{291}$. Dem Vorrang der Einzelbetreuung wird jedoch dann entsprochen, wenn das Betreuungsgericht eine Person aus dem vom Betroffenen vorgeschlagenem Verein auswählt. Nur dann, wenn der Betroffene ausdrücklich den Verein als Betreuer zugeteilt bekommen möchte, kann seinem Vorschlag aufgrund des durch die Betreuungsverfügung nicht beeinflussbaren gesetzlichen Vorrangs der Einzelbetreuung keine Bindungswirkung zugesprochen werden $^{292}$. Schlägt der Betroffene einen Betreuungsverein als die konkrete Gruppe

283 Knittel \$ 1897 Rn. 20; Perau MittRhNotK 1996, 285, 287.

${ }^{284}$ Einzelne Literaturmeinungen stufen diese Äußerung des Betroffenen nicht als konkreten Vorschlag und daher als unbeachtlich ein (Perau MittRhNotK 1996, 285, 287) oder sehen die Nennung eines Vereins oder einer Behörde nicht als Vorschlag an (Palandt/Diederichsen \1897 Rn. 20).

285 Peran MittRhNotK 1996, 285, 287.

286 Dodegge/Roth C. Rn. 140; Knittel \$ 1897 Rn. 17.

287 Knittel \$ 1897 Rn. 20; Perau MittRhNotK 1996, 285, 287.

288 Abzulehnen daher: Hausmann/Hohloch/Röthel Kapitel 14 Rn. 173 und Gernhuber/CoesterWaltjen \76 Rn. 50 - sie nehmen keine Bindungswirkung an, wenn eine abstrakte Umschreibung, z.B. „Kreis meiner Familie“"erfolgt. Gerade bei diesem Bespiel ist jedoch eine klare Umschreibung des Personenkreises erkennbar.

${ }^{289}$ Bei der beispielhaften Nennung ,mein Betreuer soll aus meiner Familie ausgewählt werde", lässt sich die (verwandtschaftliche) Familie als ein klar umschriebener Personenkreis festlegen, so dass diese eine konkrete Nennung eines Personenkreises darstellt - anders sieht dies Schwab (MünchKommBGB/Schwab \$ 1897 Rn. 21).

${ }^{290} \mathrm{Zu}$ denken ist hier sowohl an Betreuungsvereine einerseits, als auch an „einfache“, weil vom Vereinszweck mit anderen Aufgaben und Zielen betraute Sportvereine etc.

291 Siehe $\int 3$ B. II.

${ }^{292}$ Siehe dazu und zu den Ausnahmen $\ 3$ B. IV.; MünchKommBGB/Schwab \1897 Rn. 22. 
möglicher Betreuer vor, so liegt es im Ermessen des Gerichts, innerhalb des Betreuungsvereins einen ehrenamtlich tätigen Betreuer auszuwählen und somit die gesetzlichen Regeln zu wahren. ${ }^{293}$ Wird allgemein ein Verein oder eine Behörde genannt, so ist dies dahingehend auszulegen, dass der Betroffene eine Person aus diesem Verein oder dieser Behörde als Betreuer wünscht. ${ }^{294}$ Dies ist v.a. unter dem Grundsatz der Einzelbetreuung - als eine der gesetzlichen Strukturvorgaben des Gesetzes - zu werten, der nicht zur Disposition des Betroffenen steht. Der Vorschlag eines Vereins oder einer Behörde ist als wirkungslos anzusehen, weil der Betroffene ,allein auf die Person des Betreuers, nicht auf die Struktur der Betreunng Einfluss nehmen können' 295 soll.

Ist ein Personenkreis abstrakt beschrieben ${ }^{296}$ entspricht dies nach ganz herrschender Meinung nicht mehr \ 1897 Abs. 4 S. 1.297 Das Gesetz verlange einen konkreten Vorschlag, der die Person des Wunschbetreuers klar benenne. Diese Gesetzesauslegung ist fraglich. Mit der Benennung eines abstrakten Personenkreises bringt der Volljährige zum Ausdruck, dass sein Wunschbetreuer aus diesem Personenkreis auszuwählen ist. Der Wunsch des Betreuten ist damit konkret ermittelbar und daher ebenfalls nach \ 1897 Abs. 4 S. 1 verbindlich.

\section{Gegenbetreuer}

Der Gegenbetreuer wird im Betreuungsrecht nicht ausdrücklich, sondern nur durch den Verweis in $\int 1908$ i Abs. 1 auf $\iint 1792,1799$ geregelt. Eine vollständige Verweisung auf das Vormundschaftsrecht fehlt. Die herrschende Literaturmeinung beschreibt die bruchstückartige Verweisung als Redaktionsversehen aufgrund der nachträglichen gesetzlichen Einführung des Gegenbetreuers. ${ }^{298}$

\footnotetext{
${ }^{293}$ Zur Problematik der Nennung ehrenamtlich Tätiger vor Berufsbetreuern, \ 3 B. IV.

${ }^{294}$ Langenfeld S. 159; als Möglichkeit: Rudolf/Bittler \ 3 Rn. 15; Dodegge/Roth C. Rn. 138.

295 Dodegge/Roth C. Rn. 138.

296 Wie z.B. die Nennung „einer meiner Freunde“, weil die Eigenschaft der Freundschaft nicht gesetzlich festgelegt, sondern durch individuell definierte, ganz unterschiedliche Kriterien bestimmt ist und der Personenkreis somit durch Auslegung durch den Betreuungsrichter nur schwer bis gar nicht bestimmbar ist.

${ }^{297}$ MünchKommBGB/Schwab \1897 Rn. 23; Knittel \$ 1897 Rn. 18a mit dem Beispiel „Familienangehöriger": Die Familie stelle einen klar umschriebenen Kreis dar, so dass ihre Nennung als konkrete Benennung eines Personenkreises gelte. Dieser Auslegung ist so nicht zuzustimmen. Vielmehr muss der Betreuungsrichter die Nennung des Betroffenen auslegen und den Willen des Betroffenen ermitteln. Er muss dann eine in seinen Augen geeignete Person aus diesem Kreis und daher für ihn durch Auslegung konkret bestimmbaren Kreis auswählen. Problematisch und damit möglicherweise abstrakt kann die Nennung werden, wenn der Betroffene unter Familienangehörigen einen anderen Kreis als den gesetzlich bestimmten meint, wie z.B. auch den Lebensabschnittsgefährten. Ist aber auch dieser Kreis durch den Betreuungsrichter durch Auslegung konkret zu ermitteln, handelt es sich um eine ausreichend konkrete und somit verbindliche Benennung durch den Betroffenen.

298 Spanl Rpfleger 1992, 142, 144; Bienwald/Sonnenfeld/Hoffmann \1896 Rn. 156.
} 
Der Gegenbetreuer soll zum einen den Betreuer beaufsichtigen, indem er Rechenschaftslegung von diesem verlangt ${ }^{299}$ (sinngem. \ 1799 Abs. 2 i.V.m. \1908i Abs. 1 S. 1). Dies ist sonst alleinige Aufgabe des Betreuungsgerichts. Zum anderen soll er an der Verwaltung des Betreutenvermögens mitwirken ${ }^{300}$, vgl. \ $1908 \mathrm{i}$ Abs. 1 S. 1 i.V.m. SS 1792 Abs. 2, 1802 Abs. 1 S. 2. Der Gegenbetreuer erhält seinen Auftrag also nicht vom Betreuungsgericht, ${ }^{301}$ sondern direkt aus dem Gesetz, vgl. \1908i i.V.m. \1799. Er wird bestellt, wenn mit der Betreuung eine nicht unerhebliche Vermögensverwaltung verbunden ist und hat eine Kontrollfunktion.

Die Situation der Nennung des Wunsch-Gegenbetreuers durch den Betroffenen unterscheidet sich kaum zu der des Wunschbetreuers. Im Vergleich hat der Gegenbetreuer keinen eigenen Aufgabenkreis, sondern ist eine reine zur Betreuung akzessorische Kontrolleinrichtung. ${ }^{302}$ Dadurch ist er nicht zur Vertretung des Betreuten berechtigt, auch nicht bei Verhinderung des Betreuers. Bei seinem Handeln ist er dem Betreutenwohl verpflichtet. Somit sind einige strukturelle Unterschiede auszumachen, die eine differenzierte Bewertung der Vorschläge des Betroffenen jedoch nicht rechtfertigen. Folglich muss die Benennung des Gegenbetreuers analog zu der Benennung des Betreuers durch den Betroffenen nach $\int 1897$ Abs. 4 S. 1 mit verbindlicher Geltung für das Betreuungsgericht festzulegen sein. ${ }^{303}$

\footnotetext{
299 Bienwald/Sonnenfeld/Hoffmann \1896 Rn. 156.

300 Bienwald/Sonnenfeld/Hoffmann \1896 Rn. 158.

301 Wie dies aufgrund des Erforderlichkeitsgrundsatzes bzgl. der Aufgabenkreiszuteilung bei der normalen Betreuung der Fall ist.

302 Bienwald/Sonnenfeld/Hoffmann \1896 Rn. 168. Daher hat der Gegenbetreuer auch keinen eigenen Aufgabenbereich (Spanl Rpfleger 1992, 142, 144).

303 Die Grenze bildet hier allein das Wohl des Betreuten, vgl. $\$ 2$ C. III.; Perau will nur Rücksicht auf diese Vorschläge nehmen, da ein verbindlicher Vorschlag an dieser Stelle die rechtliche Ausgestaltung der Betreuung bedeute, was nicht von der Befugnis, einen Wunschbetreuer zu benennen, umfasst sei (Perau MittRhNotK 1996, 285, 287).
} 


\section{Ersatzbetreuer}

Das Gesetz enthält für die Benennung eines Ersatzbetreuers ${ }^{304}$ keine Vorgaben. Der Ersatzbetreuer wird nach \1899 Abs. 4 nicht gemeinschaftlich oder neben einem weiteren Betreuer, sondern nur für den Fall bestellt, dass der vorrangige (eigentliche) Betreuer verhindert, ein Handeln aber zum Wohl des Betreuten erforderlich ist. ${ }^{305}$ Diese Verhinderung kann sich dabei aus rein tatsächlichen ${ }^{306}$ wie rechtlichen ${ }^{307}$ Gründen ergeben.

Ähnlich wie bei der Bestimmung eines Gegenbetreuers sind hier weder ausdrückliche Regelungen des Gesetzes noch inhaltliche Unterschiede in Bezug auf die Benennung eines Wunschbetreuers nach $\int 1897$ Abs. 4 S. 1 auszumachen. Der Vorschlag des Betroffenen bezüglich seines Wunsch-Ersatzbetreuers muss somit entsprechend \1897 Abs. 4 S. 1 ebenfalls verbindlich sein.

\section{Kontrollbetreuer}

Hat der Betroffene einer dritten Person eine Vorsorgevollmacht erteilt, kann zur Geltendmachung seiner Rechte gegenüber dem Vollmachtnehmer ein Kontrollbetreuer bestellt werden, vgl. \1896 Abs. 3. Eine ausdrückliche Regelung über die Voraussetzungen der Kontrollbetreuerbestellung ist nicht vorhanden.

Aus \ 1896 Abs. 3 und der Gesetzesbegründung 308 ergibt sich einerseits die Aufgabe des Kontrollbetreuers, den Bevollmächtigten darin zu kontrollieren, ob dessen Entscheidungen dem Wohl des Betreuten zuwiderlaufen ${ }^{309}$ und andererseits die Rechte des Betreuten gegenüber seinem Bevollmächtigten wahrzunehmen. ${ }^{310}$

\footnotetext{
304 Auch Ergänzungsbetreuer genannt, mit der Bezeichnung der Ergänzung wird m.E. jedoch nicht wirklich deutlich, dass der Ersatzbetreuer nur bei Verhinderung des Betreuers nötig wird - also als Ersatz „an Stelle von“ nicht hingegen als ein zusätzlicher Betreuer „neben“ dem regulären Betreuer; vgl. auch Alperstedt BtPrax 2001, 106 ff. und Spanl Rpfleger 1992, 142 ff.

305 Dodegge/Roth B. Rn. 78; LG Stuttgart BtPrax 1999, 200; LG Frankfurt/Oder FamRZ 1999, 1221, 1222.

306 Wie bei Krankheit, Urlaub o.ä.; möglich ist dann z.T., dass der Betreuer selber einen Vertreter beauftragt, was jedoch ein Ausnahmelösung darstellt, weil die Verpflichtung des Betreuers aus \1901 grundsätzlich nicht übertragbar ist, vgl. nur Bienwald/Sonnenfeld/Hoffmann \1899 Rn. 38; Dodegge/Roth B. Rn. 80 - solange persönliche Betreuung gewährt wird; Jürgens/Jürgens $\int 1899$ Rn. 6.

307 D.h. die Vertretungsmacht nicht ausüben (\$S 1795, 1796 i.V.m. 1908i Abs. 1, 181) oder das dem Betreuten Zugewendeten nicht verwalten darf (\$S 1803 i.V.m. 1908i Abs. 1 S. 1; vgl. Dodegge/Roth B. Rn. 79; BayObLG BtPrax 1998, 32, 33) bzw. die Angelegenheit nicht auf den Verfahrenspfleger übertragen kann.

308 BT-Drucks. 11/4528 S. 123.

${ }^{309}$ Dodegge/Roth A. 26.

310 BT-Drucks. 11/4528 S. 123; Dodegge/Roth A. 26; Bienwald Rpfleger 1998, 231, 232, 234.
} 
Voraussetzung für eine Kontrollbetreuerbestellung ist nach \ 1896 Abs. 3, dass eine Vollmacht wirksam erteilt wurde und nicht erloschen ist. ${ }^{311}$ Darüber hinaus bedarf es keiner Anzeichen dafür, dass der Bevollmächtigte nicht im Interesse des Betroffenen, 312 bzw. nicht nach den im Innenverhältnis (der Bevollmächtigung) vereinbarten Kriterien handelt. Auch müssen nicht Geschäfte des Betroffenen von erheblichem Umfang und erheblicher Schwierigkeit bestehen. ${ }^{313}$ Einige verlangen entgegen der nahen Auslegung am Gesetzeswortlaut einen konkreten Missbrauchsverdacht. ${ }^{314}$ Zudem müsse der Vollmachtgeber unvermögend sein, den Bevollmächtigten selbst zu überwachen. ${ }^{315}$

Der Kontrollbetreuer ist ein regulärer Betreuer nach \ 1897, mit einem besonderen Aufgabenbereich. ${ }^{316}$ Benennt der Betroffene in seiner Betreuungsverfügung eine bestimmte Person oder einen konkret oder abstrakt beschriebenen Kreis seiner möglichen Wunschkontrollbetreuer, muss also dasselbe gelten, wie beim regulären Vorschlag eines Wunschbetreuers nach \1897 Abs. 4 S. 1.317

II. Vorschlag gem. \1897 Abs. 4 S. 2

Schließt der Betroffene durch Wünsche in seiner Betreuungsverfügung Personen vom Amt des Betreuers aus, ist es besonders wichtig, zwischen der notwendigen Auslegung einer Betreuungsverfügung und ihren rechtlichen Grenzen zu differenzieren. Wie bereits dargelegt, ist das, was der Betroffene mit seiner Äußerung erreichen will, eine Frage der Auslegung. Ob er dieses Ziel erreicht, ist hingegen davon abhängig, inwieweit das Gericht an seinen Wunsch gebunden ist. Daher ist zu differenzieren. Lehnt der Betroffene einzelne Personen als mögliche Betreuer

311 Soergel/Zimmermann \1897 Rn. 91; Palandt/Diederichsen \ 1896 Rn. 21; BayObLG FamRZ 1993, 1249.

312 So aber: Bienwald Rpfleger 1998, 231, 233.

313 So aber: Bienwald Rpfleger 1998, 231, 233; Soergel/Zimmermann \ 1896 Rn. 89; BayObLG FamRZ 1999, 1302, 1303.

314 Str.: Soergel/Zimmermann \ 1896 Rn. 91; ,konkreten Überwachungsbedarf“ nach BayObLG FamRZ 1999, 1302, 1303 = BtPrax 1999, 151; Palandt/Diederichsen \ 1896 Rn. 21; Staudinger/Bienwald \$ 1896 Rn. 133.

315 Dodegge/Roth A. 26; BT-Drucks. 11/4528 S. 123; die Frage, ob eine Kontrollbetreuerbestellung auch dann nötig ist, wenn Familienangehörige vorhanden sind, die den Bevollmächtigten überwachen könnten ist umstritten, vgl. nur Soergel/Zimmermann \ 1896 Rn. 90 f. (dortige Fn. 201); Bienwald Rpfleger 1998, 231, 233. Die Bestellung eines Kontrollbetreuers erfolgt durch den Rechtspfleger nach $\int 14$ Abs. 1 Nr. 4 RPflG. Anders als ein regulärer Betreuer, welcher durch das Betreuungsgericht bestellt wird, wird in diesem Fall der Rechtspfleger tätig; die Entscheidung ergeht als Einheitsentscheidung des zuständigen Gerichts, (Personalentscheidung mit dem durch \ 69 FGG vorgegebenen Inhalt); Bienwald Rpfleger 1998, 231, 234; Dodegge/Roth A. 26 (dortige Fn. 78).

316 Soergel/Zimmermann $\int 1896$ Rn. 92.

317 Vgl. dazu Ausführungen unter $\int 3$ A. I.; So erfolgt das Verfahren der Betreuerbestellung nach allgemeinem Recht, vgl. Bienwald Rpfleger 1998, 231, 235. 
ab, gibt es keine sachliche Rechtfertigung dafür, diesem Wunsch eine geringere Bedeutung zuzumessen, als einem positiven Vorschlag. ${ }^{318}$

Dies sehen einige mit Blick auf die Gesetzesbegründung von \1897 Abs. 4 S. 2 anders. ${ }^{319}$ Die Gesetzesbegründung führe explizit aus, dass das Gesetz „,keine strenge Bindung des Gerichts an den Widerspruch des Betroffenen" vorsieht. Sonst könne der Betroffene durch einen Widerspruch seiner Äußerungen die Betreuung vollständig verhindern. ${ }^{320}$ Zudem sei einer ,ablebnenden Äußerung" nicht das gleiche Gewicht zuzumessen, ,wie einem konkreten Wunsch“.321

Schwab spricht sich für eine ,bindende Beachtlichkeit" des Negativwunsches aus, weil ein grundlegendes Vertrauensverhältnis zwischen Betreutem und „Negativ“betreuer schon nicht entstehen könne, wenn eine Person zum Betreuer bestellt werde, die der zu Betreuende nicht wünsche. ${ }^{322}$ Ein gutes Vertrauensverhältnis sei jedoch Grundlage einer funktionierenden Betreuung.

Den Wunsch des Betroffenen, eine bestimmte Person gerade nicht zum Betreuer $\mathrm{zu}$ benennen als unverbindlich zu bewerten, erlaubt der Wortlaut von \1897 Abs. 4 S. 2: „Schlägt der Betroffene vor, eine bestimmte Person nicht zum Betreuer zu bestellen, so soll hierauf Rücksicht genommen werden. "Während Satz 1 für das Gericht ohne Ermessensspielraum formuliert wurde, ist Satz nur als sog. SollVorschrift gefasst. Dieses Ergebnis entspricht nicht den Zielen und der Systematik des Betreuungsrechts. Demnach soll der Wunsch des Betreuten in den Grenzen des $\ 1897$ immer im Vordergrund stehen und der Entscheidungsmaßstab für den Betreuer und damit auch für das Betreuungsgericht sein. Die Frage nach der Verbindlichkeit eines negativen Wunsches ist allein die Frage nach der Bindung an einen vom Betreuten im Vorfeld geäußerten Wunsch. ${ }^{323}$ An dieser Stelle kann eine Unterscheidung zwischen Negativ- und Positivwunsch nicht stichhaltig begründet werden. Die Bestellung dieser Person zum Betreuer muss bei einer Negativnennung daher unterbleiben, gerade weil eine Bindung an die Betroffenenwünsche besteht. ${ }^{324}$

318 Lipp FS Bienwald S. 177, 183; Diese Meinung vertritt jedoch der überwiegende Teil der Literatur, siehe nur Staudinger/Bienwald \$1897 Rn. 28 und Erman/Holzhauer \$1897 Rn. 4.

319 BT-Drucks. 11/4528 S. 127; MünchKommBGB/Schwab \$ 1897 Rn. 34; Knittel \ 1897 Rn. 20; Staudinger/Bienwald \$1897 Rn. 28; Bienwald/Sonnenfeld/Hoffmann \1897 Rn. 64 f.

320 BT-Drucks. 11/4528 S. 127 f.; Staudinger/Bienwald \ 1897 Rn. 28; Bamberger/Roth/Müller \1897 Rn. 15.

321 BT-Drucks. 11/4528 S. 127; Mit fehlender Vertrauensbasis argumentiert ebenfalls Diederichsen (Palandt/Diederichsen \1897 Rn. 20), dem schließen sich Müller (Bamberger/Roth/Müller \1897 Rn. 14) und Holzhauer an (Erman/Holzhauer \$1897 Rn. 3).

322 MünchKommBGB/Schwab \1897 Rn. 34; dies sei Basis einer ,gedeihlichen Zusammenarbeit“ und auch für die Verbindlichkeit eines Positivvorschlags des Betroffenen bedeutsam, vgl. BTDrucks. 11/4528 S. 127; ebenso: Bamberger/Roth/Müller $\ 1897$ Rn. 15; Palandt/Diederichsen $\int 1897$ Rn. 20.

323 Zur Gleichwertigkeit von aktuell und im Vorfeld geäußerten Wünschen, vgl. \ 2 C. II.

${ }^{324}$ Lipp FS Bienwald S. 177, 183. 
Nennt der Betroffenen in der Betreuungsverfügung in negativer Umschreibung einen bestimmten Kreis oder eine Gruppe von Personen, gilt das Gleiche. Das Gericht darf aus diesem Personenkreis keinen Betreuer benennen. ${ }^{325}$

Kommt nach Abzug der negativ Benannten keine Person als möglicher Betreuer in Frage, ist dieser Wunsch anders zu deuten. Dann lehnt der Betroffene nicht bestimmte Personen, sondern vielmehr die Betreuung insgesamt ab. In diesem Fall bleibt dem Gericht bei der Auswahl des Betreuers kein Entscheidungsspielraum, ${ }^{326}$ es muss nicht über die Auswahl, sondern über die ablehnende Haltung des Betroffenen entscheiden. Dann muss das Gericht über die Frage der Betreuung gegen den Willen des Betroffenen, mithin der Zwangsbetreuung ${ }^{327}$ und nicht über die Bindung an den Betroffenenvorschlag entscheiden.

\section{B. Gesetzliche Anforderungen gem. $\int \mathbb{S} 1896 \mathrm{ff}$.}

$\mathrm{Zu}$ untersuchen ist, welche Fragen der Betreuerauswahl die \$S 1897 - 1900 regeln und ob sie zwingend oder dispositiv sind. Erst wenn kein, die Bestellung des Wunschbetreuers ausschließender gesetzlicher Grund vorliegt, kann der Betroffene mittels der in einer Betreuungsverfügung enthaltenen Wünsche und Vorschläge Einfluss auf die Betreuerbestellung nehmen. ${ }^{328}$ Dabei handelt es sich nicht um eine Frage der Bindungswirkung, ${ }^{329}$ sondern um eine Frage des zwingenden Rechts. ${ }^{330}$ Unterliegt das Gericht für eine Frage der Auswahl des Betreuers gesetzlich zwingenden Regelungen, sind allein diese maßgeblich, d.h. es ist keine abweichende privatautonome Regelung möglich. Solche in der Betreuungsverfügung dennoch festgehaltenen Wünsche entfalten keine Rechtswirkung. Legt das Gesetz für eine Frage der Auswahl des Betreuers nur einen Rahmen fest, ist dieser privatautonom ausgestaltbar.

Bestimmt das Gesetz keine konkreten Vorgaben, können die in der Betreuungsverfügung festgehaltenen Wünsche des Betroffenen volle Rechtswirkung

\footnotetext{
325 Siehe zur Nennung eines Kreises von Personen bzw. einer Personengruppe \ 3 A. I. 1.

326 Erman/Holz̧hauer \1897 Rn. 4; ähnlich Perau MittRhNotK 1996, 285, 287.

327 Vgl. dazu ausführlich Lipp BtPrax 2008, 51, 55 f .

328 Diese Voraussetzungen eignen sich also erst gar nicht für einen Einfluss durch die Betreuungsverfügung. Dies verkennt, trotz richtiger Schlussfolgerung Dodegge (Dodegge/Roth B. Rn. 41).

329 So aber Staudinger/Bienwald \$ 1897 Rn. 23; Bamberger/Roth/Müller \ 1897 Rn. 14; auch Damrau/Zimmermann \ 1897 Rn. 22; MünchKommBGB/Schwab 1897 Rn. 31; BayObLG FamRZ 1997, 245; für den Ausschluss von in \ 1897 Abs. 3 genannten Personen. Auch Knittel versteht dies derart, er ordnet unter $\int 1897$ Abs. 3 fallende Personen aber als ungeeignet ein, vgl. \ 1897 Rn. 6. Das BayObLG lässt eine Bindung an den Vorschlag dann entfallen, wenn der Vorgeschlagene ungeeignet ist, vgl. FamRZ 1997, 245.

330 So auch Dodegge/Roth C. Rn. 138 - in Bezug auf den Vorrang der Einzelbetreuung; Palandt/Diederichsen in Bezug auf die Voraussetzungen Eignung, $\int 1897$ Abs. 3, Übernahmebereitschaft, vgl. \ 1897 Rn. 20.
} 
entfalten. Welche der Bestellvoraussetzungen des Wunschbetreuers zu den materiellrechtlich zwingenden gehört, ist im Folgenden zu prüfen.

I. Eignung der vorgeschlagenen Person gem. \ 1897 Abs. 1

Nach \ 1897 Abs. 1 muss die gewünschte Person geeignet sein, ,Angelegenheiten des Betreuten rechtlich zu besorgen und ibn in dem hierfür erforderlichen Umfang persönlich zu betreuen".331 Der Gesetzgeber wollte die Geeignetheit des Betreuers zu einer Voraussetzung seiner Bestellung machen. ${ }^{332}$ Dies entspricht der herrschenden Meinung in Rechtsprechung und Literatur. ${ }^{333}$ Die Eignung des Betreuers ist eine zwingende Voraussetzung seiner Bestellung.

Fraglich ist, ob der Einzelne durch Wünsche in seiner Betreuungsverfügung Einfluss auf die Definition der Eignung nehmen und so festlegen kann, wann eine Person geeignet ist, seine Betreuung zu übernehmen.

Die Geeignetheit in \1897 Abs. 1 ist als unbestimmter Rechtsbegriff ${ }^{334}$ vom Gericht auszufüllen. ${ }^{335}$ Dieses muss die Anforderungen bestimmen. Aufgrund der gesetzlichen Ausrichtung auf die individuelle Betreuung hat das Gericht die Anforderungen dem konkreten Fall anzupassen. Nach dem Grundsatz der Erforderlichkeit ist gem. \1896 Abs. 2 S. 1 zu untersuchen, für welche Angelegenheiten die Betreuung im konkreten Fall erforderlich ist. Je nach Erforderlichkeit werden die notwendigen Aufgabenkreise eingerichtet. Aufgabe des Betreuers ist nach $\int 1901$ Abs. 1 a.E. die rechtliche Besorgung der in dem Aufgabenkreis anfallenden Angelegenheiten des Betreuten. Dies ist seine ihm obliegende Amtstätigkeit, die sich von der nur faktischen Tätigkeit der Fürsorge unterscheidet. ${ }^{336}$ Dabei bedarf es keiner Rechtskenntnisse des Betreuers, sondern der Fähigkeit und des Willens, ${ }^{337}$ die konkret anstehenden Aufgaben mit Hilfe bzw. für den Betreuten zu

331 Bienwald FamRZ 2000, 1314, 1315; HK-BUR/Bauer \1897 Rn. 18; Palandt/Diederichsen \ 1897 Rn. 4; Bienwald FamRZ 2000, 1314 f.; laut der Gesetzesbegründung fällt unter den Begriff der Geeignetheit eines Betreuers auch die Frage seiner Geschäftsfähigkeit, vgl. BT-Drucks. 11/4528 S. 125. Dies ist jedoch ein zu weiter Begriff der Eignung, die nur die Fähigkeit der Ausübung der rechtlichen Aufgaben und der persönlichen Betreuung umschreiben kann.

332 BT-Drucks. 11/4528 S. 125 - er sieht $\ 1897$ Abs. 1 insoweit als Generalklausel für die Bestellung des Betreuers an; Staudinger/Bienwald \$ 1897 Rn. 11.

333 Vgl. nur die Ausführungen bei Staudinger/Bienwald \$ 1897 Rn. 1.

334 Bienwald/Sonnenfeld/Hoffmann \1897 Rn. 73; Dodegge/Roth B. Rn. 35.

335 Dodegge/Roth B. Rn. 35.

336 Dodegge/Roth B. Rn. 38. Diese faktischen Tätigkeiten sind zwar wünschenswert, stehen aber zu der dem Betreuer obliegenden Rechtsfürsorge in keinem Zusammenhang (Staudinger/Bienwald \1897 Rn. 12; Bienwald/Sonnenfeld/Hoffmann \ 1897 Rn. 79). Das Wort „rechtlich“ wurde nachträglich im Rahmen des 2. BtÄndG eingeführt. Es dient zur Klarstellung der Funktion des Betreuers als gesetzlicher Vertreter in rechtlichen Angelegenheiten.

337 BayObLG FamRZ 2004, 734, 735. Darüber hinaus kommt es bei der Frage der Geeignetheit eines Betreuers auf das Vorliegen bestimmter, in diesem konkreten Einzelfall erforderlicher Fähigkeiten und die Bereitschaft an, Beratung und Unterstützung von Gericht oder den Betreuungsbehörden einzuholen (Staudinger/Bienwald \$ 1897 Rn. 16; Bienwald/Sonnenfeld/Hoffmann \1897 Rn. 73; Knittel\$ 1897 Rn. 6). Im Rahmen der Geeignetheitsprüfung kommt es nicht auf 
erledigen. ${ }^{338}$ Je nach Aufgabenbereich sind unterschiedliche fachliche Fähigkeiten erforderlich. ${ }^{339}$

Damit zeigt sich, dass die Eignung des Betreuers unter Berücksichtigung der wesentlichen Umstände des Einzelfalls zu bestimmen ist. Der Gesetzgeber wollte im Betreuungsrecht den Betreuten und seine individuell Situation in den Mittelpunkt stellen. Die Betreuung ist als staatlicher Beistand in Form der Rechtsfürsorge zu sehen, ${ }^{340}$ die durch den Betreuer ausgeführt wird, vgl. \1902. Ausgangspunkt ist dabei das subjektive Wohl des Betreuten. ${ }^{341}$ Der Betreuer muss nach der gesetzlichen Formulierung in $\int 1897$ Abs. 1 und $\ 1901$ Abs. 1 geeignet sein, die Angelegenheiten rechtlich zu besorgen und den Betreuten in dem dafür erforderlichen Umfang persönlich betreuen. Hintergrund der Forderung nach persönlicher Umsetzung der rechtlichen Aufgaben bei Notwendigkeit ist das grundsätzliche gesetzgeberische Ziel einer personenbezogenen Individualbetreuung, statt einer anonymen Verwaltung. ${ }^{342}$ Für die Definition der Eignung ist daher an dieser Stelle festzuhalten, dass der Betreuer die Besorgung der Aufgaben in personbezogener Form, also individuell erledigen können muss.

Die Ziele des Betreuungsrechts, der Sinn und Zweck des Erfordernisses der Eignung des Betreuers erlauben für den Verfasser der Betreuungsverfügung die Definition der Eignung des Betreuers. Damit ist die Eignung zwar nicht disponibel, aber der Betroffene hat indirekt Einfluss darauf, weil es um die Eignung für seine Betreuung geht. Er beschreibt sie durch seine individuellen Wünschen, denen der Betreuer entsprechen können und für deren Umsetzung und Ausführung er speziell geeignet sein muss. Zumeist ist anzunehmen, dass der Wunschbetreuer

die negative Selektion bestimmter Umstände an, so aber MünchKommBGB/Schwab \1897

Rn. 27; BayObLG FamRZ 1994, 530.

338 Dodegge/Roth B. Rn. 37; Peters S. 105 f.

339 Im Aufgabenkreis Vermögensverwaltung benötigt der Betreuer beispielsweise vertiefte Rechtskenntnisse, wenn es sich um ein großes Vermögen mit mehreren Immobilien oder der Verwaltung eines Unternehmens handelt. Für die Verwaltung eines kleinen Vermögens, die Stellung eines Rentenantrags oder die Geltendmachung von Rentenansprüchen ist dies regelmäßig nicht erforderlich, vgl. Bienwald/Sonnenfeld/Hoffmann \1896 Rn. 133. Der Aufgabenkreis Gesundheit, die Kontrolle eines Bevollmächtigten oder der Widerruf einer Vollmachtserklärung sowie die Organisation der ambulanten Versorgung erfordern vergleichsweise geringe Rechtskenntnisse.

Der Betreuer muss auch nicht deutscher Staatsangehöriger sein, vgl. Palandt/Diederichsen $\ 1897$ Rn. 4; Knittel \1898 Rn. 1; Bienwald/Sonnenfeld/Hoffmann \1898 Rn. 9 f. Es kann sogar sinnvoll sein, eine Person mit der (ausländischen) Staatsangehörigkeit des Betroffenen zu bestellen, die sich beispielsweise mit der Kultur des Betreuten auskennt, vgl. Palandt/Diederichsen $\ 1897$ Rn. 4; BT-Drucks. 11/4528 S. 129; Knittel\$1898 Rn. 1.

340 Palandt/Diederichsen Einf. v. \1896 Rn. 1.

341 Vgl. z.B. IS 1896 Abs. 1a, 1897 Abs. 4, 1901 Abs. 2, 3, 1901a, b, c sowie die Ausführungen unter $\int 2$ C. II. 2. und III.

342 Vgl. BT-Drucks. 11/4528 S. 53. Die Fähigkeit der Kommunikation ist dabei Grundvoraussetzung, da eine Individualbetreuung nur erfolgen kann, wenn die Wünsche des Betroffenen bekannt sind. 
diese Fähigkeiten hat. Aufgrund der Ausfüllungsbedürftigkeit der Geeignetheit als unbestimmten Rechtsbegriff, hat das Gericht anhand der Angaben des Betroffenen dennoch diese Prüfung vorzunehmen.

II. Vorrang der privaten, auch berufsmäßigen

Einzelbetreuung gem. \$S 1897 Abs. 1 S. 1, 1900

\section{Stufenverhältnis gem. $\iint 1897,1900$}

Das Gesetz beschreibt in $\int 1897$ und \ 1900 verschiedene Formen von Betreuungstypen. Dabei formuliert es ein Stufenverhältnis. Nach \1897 Abs. 1 ist eine natürliche Person zum Betreuer zu bestellen. Falls der Volljährige durch eine oder mehrere natürliche Personen ${ }^{343}$ nicht hinreichend betreut werden kann, bestellt das Gericht einen Verein zum Betreuer, ${ }^{344}$ vgl. \ 1900 Abs. 1. Der Verein überträgt die Wahrnehmung der Betreuung auf einzelne natürliche Personen, vgl. $\int 1900$ Abs. 2.345 Kann der Volljährige auch nicht durch einen Betreuer, der einem Verein angehört, hinreichend betreut werden, bestellt das Gericht die zuständige Behörde zum Betreuer, vgl. \ 1900 Abs. 4. Dies gilt als letztes Mittel. ${ }^{346}$ Die Bestellung eines Vereins oder einer Behörde als Betreuer soll nur im äußerten Fall und dann nur vorübergehend zulässig sein. ${ }^{347}$

Nach der Analyse der gesetzlichen Regelungen und ihrer zugrunde liegenden Begründung hat die private, auch berufsmäßige Einzelbetreuung Vorrang vor der Betreuung durch einen Vereins- oder Behördenbetreuer und vor der Betreuung durch die Betreuungsbehörde. ${ }^{348}$ Unter Einzelbetreuung ist die Betreuung durch eine natürliche Person zu verstehen. ${ }^{349}$ Die Betreuung durch einen Vereinsbetreuer bzw. einen Behördenbetreuer ist Einzelbetreuung, vgl. \ 1897 Abs. 2.350 Wird der Verein oder die Behörde als Institution zum Betreuer bestellt, ${ }^{351}$ überträgt sie die Tätigkeit der Betreuung auf einzelne Personen, vgl. \1900 Abs. 2.

\footnotetext{
343 BT-Drucks. 11/4528 S. 124.

344 BayObLG FamRZ 1993, 1248, 1249; Bienwald/Sonnenfeld/Hoffmann \ 1900 Rn. 6; MünchKommBGB/Wagenitz vor $\int 1773$ Rn. 3.

345 Vereins- oder Behördenbetreuer.

346 BayObLG FamRZ 1999, 1303; als absolute Auffangzuständigkeit/ ultima ratio, vgl. BayObLG FamRZ 1993, 1248, 1249; BayObLG FamRZ 1994, 1203; Knittel $\$ 1900$ Rn. 13; Staudinger/Bienwald S 1900 Rn. 8; Jurgeleit/Jurgeleit, \ 1900 Rn. 13; Bienwald/Sonnenfeld/Hoffmann \ 1900 Rn. 1; MünchKommBGB/Schwab S 1900 Rn. 8.

347 BT-Drucks. 11/4528 S. 131; BayObLG FamRZ 1993, 1248; BayObLG FamRZ 1999, 52; Damrau/Zimmermann S 1897 Rn. 3; Knittel S 1900 Rn. 13; Schwab FamRZ 1990, 681, 683 und 1992, 493, 499.

348 BT-Drucks. 11/4528 S. 131.

349 juris PK-BGB/Bieg/Jaschinski \1897 Rn. 6.

350 Palandt/Diederichsen \1897 Rn. 9; juris PK-BGB/Bieg/Jaschinski \1897 Rn. 6; BayObLG FamRZ 1999, 52; Jurgeleit/Jurgeleit S 1900 Rn. 2.

351 Staudinger/Bienwald \$ 1900 Rn. 1.
} 
Fraglich ist, ob der Volljährige durch Wünsche in der Betreuungsverfügung auf das gesetzlich vorgegebene Stufenverhältnis der Betreuungstypen einwirken kann.

Die Betreuung durch natürliche Personen dient dem Reformziel der persönlichen Betreuung. ${ }^{352}$ Der Betreuer steht für den Betreuten kontinuierlich als Ansprechpartner zur Verfügung. Nur bei der kontinuierlichen Betreuung durch eine bestimmte natürliche Person kann ein Vertrauensverhältnis entstehen. \1900 bestimmt, dass von der Einzelbetreuung abgesehen werden kann, wenn der Betreute durch eine oder mehrere natürliche Personen nicht hinreichend betreut werden kann. ${ }^{353}$ Die Ausnahmen von der Einzelbetreuung sind nach der Gesetzesbegründung und der eindeutigen Formulierung in $\$ 1899$ Abs. 1 und Abs. 4 nur in diesen beiden Fällen zulässig. Entweder können die Angelegenheiten des Volljährigen durch mehrere Betreuer besser besorgt werden oder der Betreuer ist aus tatsächlichen oder rechtlichen Gründen verhindert. ${ }^{354}$ Hinreichend ist somit dahingehend zu verstehen, dass die Besorgung der Betreutenangelegenheiten durch eine natürliche Person nicht mehr gewährleistet ist.

Die Einzelbetreuung wurde aus den in Vormundschaft und Pflegschaft bekannten Typen übernommen, ${ }^{355}$ vgl. \ 1791a Abs. 3, der gesetzlich angeordneten Vorrang genießt. ${ }^{356}$ Dem Gericht wird in dieser Frage kein Ermessen gewährt, es hat sich strikt an das gesetzlich vorgegebene Stufenverhältnis zu halten. ${ }^{357}$ Diese lassen ein privatautonom bestimmtes Abweichen von dem gesetzlich vorgegebenen Stufenverhältnis nicht zu. Die Disposivität der einschlägigen Normen würde einen Einschnitt in das System der gesetzlichen Betreuung bedeuten, der bedeut-

352 BT-Drucks. 11/4528 S. 50, 124; BayObLGZ 1997, 228, 290; Staudinger/Bienwald \1900 Rn. 2; Soergel/Zimmermann \ 1900 Rn. 1.

353 BT-Drucks. 11/4528 S. 126; BayObLG Beschluss vom 31.01.1997 Az.: 3Z BR 2/97; Lipp FS Bienwald S. 177, 184; Soergel/Zimmermann \1900 Rn. 2; Jurgeleit/Jurgeleit \1900 Rn. 2; BayObLG FamRZ 1999, 52; Dodegge/Roth B. Rn. 71.

${ }^{354}$ Im Einzelfall kann die Beantwortung der Frage, wann der Volljährige gem. \1900 Abs. 1 S. 1 „durch eine oder mehrere natürliche Personen nicht binreichend betreut werden kann" schwierig werden. Bei der Antwort handelt es sich um eine Ermessensentscheidung des Gerichts (Soergel/Zimmermann $\int 1900$ Rn. 2). Dabei sollte das aufgrund von Krankheit besonders komplizierte Persönlichkeitsbild des Betreuten berücksichtigt werden (BT-Drucks. 11/4528 S. 131).

355 Bienwald FamRZ 2000, 415; Leitbild im Vormundschaftsrechts des BGB war der Einzelvormund, der im Regelfall allein einen oder zwei Mündel betreute, vgl. \1786 Abs. 1 Nr. 8, Staudinger/Engler \1791a Rn. 1, 10; Gernhuber/Coester-Waltjen \76 Rn. 41; Bereits in den Motiven zum BGB lässt sich der Grundsatz der Einzelbetreuung nachlesen, demnach war - allerdings aus Kontrollaspekten - ,für jede Vormundschaft nur ein verwaltender Vormund zu bestellen", nur „,Bei größeren und schwierigeren Verwaltungen kann ein Bedürfnis [...] für mehrere Vormünder vorbanden sein." vgl. Motive zum BGB, Vormundschaft, Allgemeine Begründung (\$S 1633 - 1748) V. Verwaltender Vormund. Gegenvormund.

356 MünchKommBGB/Wagenit: \1791a Rn. 2.

357 BayObLG BtPrax 1994, 171, 172; BayObLG Beschluss vom 17.07.1996, Az.: 3Z BR 159/96 (unveröffentlicht); BayObLG Beschluss vom 31.01.1997 Az.: 3Z BR 2/97 (unveröffentlicht); Bienwald/Sonnenfeld/Hoffmann $\$ 1900$ Rn. 2. 
same Grundmaximen des Rechtsinstitutes berühren würde. Der Grundsatz der Einzelbetreuung kann somit nicht mittels einer Betreuungsverfügung privatautonom aus- und umgestaltet werden. ${ }^{358}$

\section{Auswabl von Vereinsmitglieder gem. \1900 Abs. 2 S. 2}

$\int 1900$ Abs. 2 regelt die Wahrnehmung einer Betreuung durch einen Verein. Dabei bestimmt S. 2, dass der Verein bei der Übertragung der Betreuung auf einzelne Personen „Vorschlägen des Volljährigen [...] zu entsprechen hat, soweit nicht wichtige Gründe entgegenstehen. "Nach der Gesetzesbegründung soll nach Satz 2 dem Willen des Volljährigen hingegen - entgegen dem eindeutigen Wortlaut des Gesetzes nur Beachtung geschenkt, aber nicht zwangsläufig Vorrang gewährt werden. ${ }^{359}$ Ein \1897 Abs. 5 entsprechender Willensvorrang könne zu organisatorischen Schwierigkeiten führen. 360 Satz 2 sei demnach nur als „Ausdruck" des in $\ 1897$ Abs. 4 und \ 1901 Abs. 3 ausdrücklich gesetzlich normierten Wunschvorrangs des Betreuten zu bewerten. ${ }^{361}$ Dieser Auslegung kann nicht gefolgt werden. Es wird nicht deutlich, warum der Vorschlag des Betreuten keine \1897 Abs. 4 entsprechende verbindliche Wirkung haben sollte. Nach dem Gesetzestext steht dem Verein dann keine Möglichkeit zu, von dem Betroffenenwunsch abzuweichen, wenn dem wichtige Gründe entgegenstehen. Rein organisatorische Probleme dürfen darunter nicht verstanden werden. ${ }^{362}$ Sie sind nicht gleichbedeutend mit der Frage nach der rechtlichen Verbindlichkeit des Betroffenenwunsches. Bis zu dieser Grenze sind die Betroffenenwünsche vielmehr als verbindlich anzusehen. Eine andere Wertung würde der systematischen Einordnung in Bezug auf \ 1897 Abs. 4 nicht entsprechen. Gründe, von dieser Gesetzessystematik abzuweichen sind nicht ersichtlich. Die Auswahl eines einzelnen Vereinsmitgliedes ist für den Betroffenen somit möglich.

III. Ausschluss der in $\int 1897$ Abs. 3 genannten Personen

Gem. \1897 Abs. 3 darf eine Person ,nicht zum Betreuer bestellt werden," die zu „einer Anstalt, einem Heim oder einer sonstigen Einrichtung, in welcher der Volljährige untergebracht ist oder wohnt, in einem Abhängigkeitsverhältnis oder einer anderen engen Beziehung steht". Dieser Ausschluss des Weisungsabhängigen sieht keine gesetzliche Ausnahme vor, ${ }^{363}$ so dass für das Gericht im Rahmen der Entscheidungsfindung kein Ermes-

358 Ebenso: Lipp FS Bienwald S. 177, 184; MünchKommBGB/Schwab \ 1897 Rn. 22; Staudinger/Bienwald \1900 Rn. 8; Jürgens/Jürgens \1900 Rn. 2; BayObLG Rpfleger 1998, 199; Bay-

ObLG FamRZ 1999, 52.

${ }^{359}$ BT-Drucks. 11/4528 S. 132.

360 BT-Drucks. 11/4528 S. 132.

361 Soergel/Zimmermann \1900 Rn. 11.

362 Vgl. nähere Ausführungen unter \2 C. III.

363 BT-Drucks. 11/4528 S. 126; Damrau/Zimmermann \1897 Rn. 22; Staudinger/Bienwald \ 1897 Rn. 23; HK-BUR/Bauer $\$ 1897$ Rn. 51; OLG Stuttgart FamRZ 1999, 812; widersprüchlich hier BayObLG FamRZ 1997, 245. 
sen besteht. ${ }^{364}$ Der Vorschlag des Bundesrates, die Vorschrift als Soll-Vorschrift zu fassen, wurde vom Gesetzgeber nicht übernommen. Dieser wollte ein klares Verbot. 365

Nach der Gesetzesbegründung sollen durch diese Regelung primär Interessenskonflikte vermieden, ${ }^{366}$ aber auch sichergestellt werden, dass der Betreuer gegenüber der Einrichtung in der sich der Betroffene aufhält unvoreingenommen ist. ${ }^{367}$ Der Gesetzgeber will so alle denkbaren Interessenskonflikte zwischen dem Betreuer und dem Betreuten, der Leitung sowie dem Personal der Einrichtung, in der der Betroffene lebt, ausschließen. ${ }^{368}$ Literatur und Rechtsprechung legen die Norm entsprechend dieser Gesetzesbegründung aus. ${ }^{369}$ Danach reicht auch die abstrakte Gefahr, die sich aus dem Abhängigkeitsverhältnis eines Dritten ergeben kann. ${ }^{370}$ Sie stufen die Vermeidung eines möglichen Interessenkonflikts sowie mögliche Zweifel an der Unvoreingenommenheit dieser Weisungsabhängigen so hoch ein, dass Abs. 3 vermehrt als absoluter Ausschlussgrund einer Betreuerbestellung definiert wird. ${ }^{371}$ Die Wirkung eines Betroffenenvorschlags entfiele bei Vorliegen der benannten Abhängigkeitsverhältnisse. ${ }^{372}$

Unter einem Heim, einer Anstalt oder einer sonstigen Einrichtung sind solche Kliniken, Krankenhäuser, Pflegeheime und Altenwohnanlagen zu verstehen, die nicht als Privatwohnung genutzt werden. ${ }^{373}$ Nach dem Gesetzeszweck ist dies weit auszulegen. ${ }^{374}$ Unter „Wohnen“ versteht man das tatsächliche Nutzen privater

364 Dodegge/Roth B. Rn. 41; MünchKommBGB/Schwab \1897 Rn. 31; Damrau/Zimmermann \ 1897 Rn. 22; HK-BUR/Bauer \ 1897 Rn. 51; Palandt/Diederichsen S 1897 Rn. 15; BT-Drucks. 11/4528

S. 126; BayObLG FamRZ 1997, 245; BayObLG FamRZ 1999, 50.

365 BT-Drucks. 11/4528 S. 226; BT-Drucks. 11/4572 S. 207; BT-Drucks. 11/6949 S. 72.

366 BT-Drucks. 11/4528 S. 126; HK-BUR/Bauer $\int 1897$ Rn. 50.

367 BT-Drucks. 11/4528 S. 126; HK-BUR/Bauer $\$ 1897$ Rn. 50.

368 HK-BUR/Bauer \ 1897 Rn. 51; OLG Düsseldorf FamRZ 1994, 1416; OLG München FamRZ 2006, 442.

${ }^{369}$ Lipp FS Bienwald S. 177, 184; Epple BWNotZ 1992, 27, 28; MünchKommBGB/Schwab \ 1897 Rn. 31.

370 Dodegge/Roth B. Rn. 41; OLG Düsseldorf FamRZ 1994, 1416: Eine abstrakte Gefahr ist schon dann gegeben, wenn der Ehemann der als Betreuer in Erwägung gezogenen Person in einem Abhängigkeitsverhältnis zu der Einrichtung steht, in der sich der Betroffene aufhält.

371 BayObLG FamRZ 1997, 245; Bienwald/Sonnenfeld/Hoffmann \1897 Rn. 29; MünchKommBGB/Schwab S 1897 Rn. 31.

372 OLG Düsseldorf FamRZ 1994, 1416; BayObLG FamRZ 1997, 245; Bienwald/Sonnenfeld/Hoffmann \ 1897 Rn. 29; Erman/Holz̧auer \ 1897 Rn. 11; MünchKommBGB/Schwab \ 1897 Rn. 23; Jürgens/Kröger/Marschner/Winterstein Rn. 111b; Damrau/Zimmermann \ 1897 Rn. 22.

373 Siehe ausführliche Auflistung bei HK-BUR/Bauer \ 1897 Rn. 52 ff.; die Grenze ist bei der privaten Wohnung zu ziehen, auch wenn diese im Eigentum von Angehörigen steht oder aber von diesen gemietet wurde (Damrau/Zimmermann \ 1897 Rn. 23), weil die Familienpflege von Abs. 3 nicht betroffen sein soll.

374 Andernfalls könnten Familienmitglieder Betreuungen nicht übernehmen, was den Grundsätzen des Betreuungsrechts widerspräche, vgl. \1897 Abs. 5 der verlangt, verwandtschaftliche Beziehungen bei der Einrichtung der Betreuung besonders positiv zu berücksichtigen (HK- 
Räumlichkeiten. ${ }^{375}$ „Untergebrachtsein“ ist nach dem Zweck der Norm dem Wohnen zwar ähnlich, aber weiter zu fassen. ${ }^{376}$ Von einem Abhängigkeitsverhältnis des möglichen Betreuers zu der in Frage stehenden Einrichtung ist dann auszugehen, wenn zwischen beiden ein Arbeitsverhältnis ${ }^{377}$ bzw. allgemein eine Weisungsgebundenheit ${ }^{378}$ besteht. Eine sonstige enge Beziehung schließt die Befähigung zum Betreueramt ebenfalls aus. An das Fehlen einer Weisungsgebundenheit sind strenge Anforderungen zu stellen. ${ }^{379}$ Es wird absolute „Unverbundenheit“ gefordert. Sogar Verwandte von in einem Arbeitsverhältnis Stehenden sind von einer Übernahme der Betreuung ausgenommen, wenn sie zu dem Abhängigen in enger persönlicher Beziehung stehen. ${ }^{380}$ Leiter einer Einrichtung sind schon aufgrund der besonders großen abstrakten Gefahr ausgeschlossen. ${ }^{381}$ Eine Gefahr bestehe auch dann, wenn die Betreuung für andere Aufgabenkreise als die Unterbringung und die Aufenthaltsbestimmung eingerichtet wurde. ${ }^{382}$ Dies gilt auch, wenn Konflikte hauptsächlich den Aufenthalt der Person ${ }^{383}$ sowie die Vermögenssorge ${ }^{384}$ betreffen.

BUR/Bauer \1897 Rn. 51b, 53; MünchKommBGB/Schwab \1897 Rn. 32; Damrau/Zimmermann \ 1897 Rn. 23).

375 HK-BUR/Bauer \1897 Rn. 52; Damrau/Zimmermann \1897 Rn. 24.

376 HK-BUR/Bauer $\$ 1897$ Rn. 52b. Auch ein vorübergehender Aufenthalt kann daher erfasst sein.

Einige fordern hier eine Mindestfrist von sechs Wochen um die Abgrenzung zu einem vorübergehenden Aufenthalt beispielsweise in einem Krankenhaus zu gewährleisten. Eine Freiheitsentziehung kann, muss aber nicht damit verbunden sein.

377 HK-BUR/Bauer \1897 Rn. 54; Damrau/Zimmermann \1897 Rn. 25; Bienwald sieht hingegen die Sorge, dass der Betreuer sich bei Fragen im Rahmen seiner Betreuertätigkeit nicht gegen seine Arbeitgeber durchsetzen könne, als unbegründet an (Bienwald Rpfleger 1998, 231, 233).

378 HK-BUR/Bauer \ 1897 Rn. 54; disziplinarisch unterstellt nach Staudinger/Bienwald \1897 Rn. 23.

379 So reicht es nicht, dass einerseits Betreuung und Heimleitung organisatorisch getrennt und andererseits die Weisungsgebundenheit arbeitsrechtlich gesichert ist, vgl. Damrau/Zimmermann \1897 Rn. 22.

380 Damrau/Zimmermann \1897 Rn. 22; Staudinger/Bienwald \1897 Rn. 23; Dodegge/Roth B. Rn. 41; OLG Düsseldorf FamRZ 1994, 1416; BayObLG FamRZ 1997, 245, 246; BayObLG FamRZ 1999, 50; BVerfG NJW-RR 2006, 1009 wobei dies explizit im Einzelfall zu prüfen ist. Eine Betreuungsübernahme ist sicher dann nicht möglich, wenn nicht ausgeschlossen werden kann, dass der mögliche Betreuer bei der Durchsetzung der Betroffeneninteressen gegen die Einrichtung Rücksicht auf die Situation seines Angehörigen nimmt, vgl. MünchKommBGB/Schwab \ 1897 Rn. 33; andere fordern eine enge Beziehung zur Einrichtung selbst, vgl. OLG Stuttgart FamRZ 1999, 812; BayObLG FamRZ 1997, 245, 246; BayObLG BtPrax 1998, 76; Palandt/Diederichsen \1897 Rn. 14.

381 BVerfG NJW-RR 2006, 1009 f.; HK-BUR/Bauer \1897 Rn. 51b; ist der Leiter oder Inhaber einer Einrichtung hingegen Elternteil, muss dies als Sonderkonstellation bewertet werden. Die Gefahr des Interessenkonflikts ist dann nicht ausgeschlossen, sie liegt aber wesentlich ferner bei engen verwandtschaftlichen Beziehungen.

382 Epple BWNotZ 1992, 27, 28.

383 Bzw. deren Aufenthaltswechsel und allgemein der Durchsetzung von Rechten des Betreuten gegen die Einrichtung, vgl. BVerfG FamRZ 2006, 1509, 1510; BT-Drucks. 11/4528 S. 126.

384 V.a. finanziellen Verpflichtungen ggü. dem Heim, vgl. BT-Drucks. 11/4528 S. 126. 
Das Ziel, mögliche Interessenskonflikte zu vermeiden und die Unvoreingenommenheit des Betreuers zu wahren, sieht das Gesetz vor, um das Verhältnis zwischen Betreuer und Betreuten zu schützen. Wie bereits aufgezeigt, ist das Vertrauensverhältnis des Betreuten gegenüber dem Betreuer für die erfolgreiche Betreuung Grundvoraussetzung. ${ }^{385}$ Auf das Verbot des \ 1897 Abs. 3 kann der Volljährige mittels individueller Wünsche in der Betreuungsverfügung nicht einwirken. Wenn der Heimleiter als langjähriger Familienfreund eine Person seines Vertrauens ist, verbietet \ 1897 Abs. 3 seine Bestellung zum Betreuer. \ 1897 Abs. 3 ist zwingendes Recht und als solches unabdingbar.

IV. Ehrenamtliche Betreuung gem. \1897 Abs. 6 S. 1

Gem. \1897 Abs. 6 soll, „wer Betreuungen im Rabmen seiner Berufsausübung führt [...] nur dann zum Betreuer bestellt werden, wenn keine andere geeignete Person zur Verfügung steht, die zur ebrenamtlichen Führung der Betreunng bereit ist. "Fraglich ist, ob diese gesetzliche Regelung den Volljährigen in der Wahl seines Wunschbetreuers einschränkt. Kann ein Vorschlag, wenn die gewünschte Person Betreuungen von Berufs wegen ausführt, rechtlich unbeachtlich sein, wenn ein geeigneter ehrenamtlicher Betreuer zur Verfügung steht?

$\int 1897$ Abs. 6 wurde durch das 1. BtÄndG auf Vorschlag des Bundesrats ${ }^{386}$ eingefügt. Der ehrenamtlichen Betreuung sollte nach der Gesetzesbegründung grundsätzlich der Vorrang vor der Berufsbetreuung gebühren. Die vorherigen gesetzlichen Regelungen regelten bereits eingehend die Rangfolge bei der Bestellung des Betreuers, brachten diesen Grundsatz nach Auffassung des Bundesrates jedoch nicht hinreichend zum Ausdruck. ${ }^{387}$

Mit dieser Rangfolge sollte v.a. die Bestellung überqualifizierter Betreuer vermieden werden. Der Hintergrund dieses Bestrebens lag zum einen in der Schonung der Staatskasse. ${ }^{388}$ Grundsätzlich ist nach den Regeln des Betreuungsrechts die Vergütung eines Berufsbetreuers beziehungsweise der Aufwendungsersatz eines ehrenamtlich tätigen Betreuers gem. \1908i Abs. 1 i.V.m. IS 1835, 1835a, 1836 aus dem Vermögen des Betreuten zu begleichen. ${ }^{389}$ Bei Mittellosigkeit des Betreuten wird die Vergütung aus der Staatskasse gewährt. Dem Staat steht dann

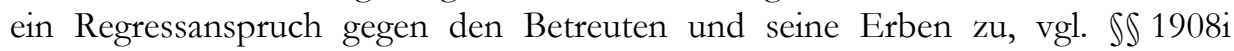
Abs. 1, 1835, 1836, 1836e. Dann haften die Erben für den Rückgriffanspruch des Staates, allerdings beschränkt auf den Wert des Nachlasses zum Zeitpunkt des Todes des Betreuten und nur innerhalb einer Frist von 10 Jahren, vgl. \1836e. 390

\footnotetext{
385 So auch schon BT-Drucks. 11/4528 S. 129.

386 BT-Drucks. 13/7158 S. 49, 57.

387 BT-Drucks. $13 / 7158$ S. 50.

388 BT-Drucks. $13 / 7158$ S. 50.

389 Palandt/Diederichsen \1835 Rn. 1; MünchKommBGB/Wagenitz』1835 Rn. 6 ff.; s.a. \5 B.

390 Dieser Regressanspruch ist jedoch insoweit erfolglos, als dass der mittellose Betreute auch kein Erbe haben wird, aus dem der Anspruch erfüllt werden könnte.
} 
Zum anderen wollte der Gesetzgeber mit diesem Ziel Betreuer mit besonderer Qualifikation dem Einsatz für diejenigen Betroffenen vorbehalten, welche die entsprechenden Kenntnisse und Fähigkeiten des Betreuers wirklich benötigten. 391

Nach der Rechtsprechung soll ein \ 1897 Abs. 6 entgegenstehender Wunsch des Betreuten bis auf zwei Ausnahmen hinter den strikten gesetzlichen Vorgaben zur Rangfolge der Betreuer zurücktreten. ${ }^{392}$ Die erste Ausnahme wird angenommen, wenn der benannte Wunschbetreuer eine besonders enge Vertrauensperson des Betroffenen ist, die zweite Ausnahme, wenn er vermögend ist. ${ }^{393}$ Die Literatur folgt dem in weiten Teilen. ${ }^{394}$ Bei Geeignetheit ${ }^{395}$ des ehrenamtlich tätigen Betreuers will eine verbreitete Literaturmeinung diesen bestellen, auch wenn dies dem Wunsch des Betroffenen widerspricht, ${ }^{396}$ lässt aber ebenfalls beide Ausnahmen zu.

Eine Begrenzung der freien Betreuerauswahl des Betroffenen durch den Grundsatz des Vorrangs der ehrenamtlichen Betreuung wird nur dann anzunehmen sein, wenn der Wunschbetreuer zufällig (auch) Berufsbetreuer ist. Der demographische Wandel und die damit zunehmende Zahl von Betreuungen lassen Bekanntschaften zu Betreuern aus bestehenden Betreuungen befreundeter Mitbürger häufiger werden. Wurde in der Familie einmal eine Betreuung zur Zufriedenheit aller Beteiligten im Rahmen einer Berufsausübung geführt, greifen die Angehörigen gerne auf diesen zurück. Das bestehende Vertrauensverhältnis zwischen den Beteiligten dient als Arbeitsgrundlage. Der Vorrang der ehrenamtlichen Betreuung zum Schutz der Staatskasse spiegelt fiskalische Interessen wider und kann nicht als Argument herangezogen werden, den - nach allgemeinen Grundsätzen verbindlichen ${ }^{397}$ - Vorschlag des Betreuten zu missachten. ${ }^{398}$ Gerade wenn der „erwünschte“ Betreuer zu dem Betroffenen bereits in einem bereits länger andauernden

\footnotetext{
391 BT-Drucks. 13/7158 S. 50.

${ }^{392}$ Nach dem 6. ZS des OLG Jena im Beschluss vom 18.09.2000 Az.: 6 W 489/00 FamRZ 2001, 714 f. gilt Folgendes: ,Aus \1897 Abs. 6 S. 1 folgt ebenso wie aus einem Umkebrscbluss aus $\int 1897$ Abs. 6 S. 2 und aus $\int 1908$ b Abs. 1 S. 2, dass der Gesetzgeber des Bt ÄndG der ebrenamtlichen Betreunng bewusst den Vorrang gegeben hat vor der beruflich geführten Betreuung. Das Betreuungsgericht hat grundsätzlich diesen Vorrang auch gegenüber einem durch den Betreuten eingebracbten Vorschlag zu beacbten. ", so auch: OLG Jena FamRZ 2001, 714 f.; KG Berlin Rpfleger 2006, 651 ff.

${ }^{393}$ Erman/Holzhauer \$1897 Rn. 9; Dodegge/Roth B. Rn. 52; Knittel\$ 1897 Rn. 23a; Palandt/Diederichsen $\int 1897$ Rn. 9.

${ }^{394}$ Der Vorschlag des Betroffenen ist nur in den zwingenden Grenzen des $₫ 1897$ Abs. 4 und 6 bindend, vgl. MünchKommBGB/Schwab \ 1897 Rn. 40; anders dazu: Lipp FS Bienwald S. $177,184$.

395 Wobei in der Rechtsprechung bei dieser Prüfung nicht die Geeignetheit im engen, sondern im weiten Sinn als allgemeine Voraussetzung gemeint ist, vgl. § 3 B. I.

${ }^{396}$ Erman/Holzhauer \1897 Rn. 9; Bamberger/Roth/Müller \ 1897 Rn. 18; Palandt/Diederichsen \1897 Rn. 9; Staudinger/Bienwald \ 1897 Rn. 28; Knittel \$ 1897 Rn. 23a - jedoch mit der Meinung, dass der Nachrang eines Berufsbetreuers jedenfalls dann zu beachten sei, wenn er wesentlich besser geeignet ist. Anderer Ansicht ist Schwab (MünchKommBGB/Schwab \1897 Rn. 22). 397 Siehe dazu ausführlich oben, $\mathbb{\Omega} 2$.

398 Bamberger/Roth/Müller $\$ 1897$ Rn. 18.
} 
persönlichen Kontakt steht, ${ }^{399}$ kennt er dessen persönliche Einstellungen, Vorlieben und Ansichten und kann somit durch sein Wissen um Charakter, Lebenslauf, familiärer und sozialer Situation, seinen Wunsch am besten in seinem Verständnis umsetzen. Aufgrund der Wunschbeachtungspflicht des \1901 Abs. 2 und 3, sowie des Selbstbestimmungsrechts des Betroffenen, ist \ 1897 Abs. 6 daher dahingehend zu verstehen, dass er für den Berufsbetreuer die Möglichkeit bietet, eine Betreuung ehrenamtlich zu führen. ${ }^{400}$ Darüber hinaus wird der Betroffene nach der Gesetzesbegründung, der herrschenden Meinung in Rechtsprechung und Literatur bis auf die zwei aufgezeigten Ausnahmen an den Vorrang der ehrenamtlichen Betreuung gebunden sein.

\section{V. Übernahmepflicht und Bereiterklärung gem. \1898}

Dem Betreuer muss nach $\int 1898$ Abs. 1 die Übernahme der Betreuung zumutbar sein. Nach Satz 2 muss er sich dazu bereit erklären. Fraglich ist, ob der Betroffene aufgrund $\int 1901$ Abs. 3 S. 1 durch seinen Wunsch in der Betreuungsverfügung das $\mathrm{Maß}$ der Übernahmepflicht verändern und die Bereiterklärung ersetzen kann; also durch die Nennung in der Betreuungsverfügung eine Pflicht des Benannten normiert.

Satz 1 formuliert ein Maß der Zumutbarkeit. Die Übernahme einer Betreuung ist dem Betreuer dann zumutbar, wenn dies „unter Berücksichtigung seiner familiären, beruflichen und sonstigen Verhältnisse" möglich ist" ${ }^{401}$, vgl. \ 1898 Abs. 1 S. 2. Nach der Gesetzesbegründung sollte eine Kasuistik der Unzumutbarkeitsgründe v.a. mit Blick auf $\int 1786$ bewusst nicht geschaffen werden. ${ }^{402}$ Nur durch fehlende starre Vorgaben kann die Zumutbarkeitsgrenze für den individuellen Fall bestimmt werden. Geringe Anforderungen bewirken, dass die Betreuung von vielen Personen

399 Die ehrenamtliche Betreuung (ca. $96 \%$ aller Betreuungen), übernehmen zu $80 \%$ nahe stehende Personen, zu 7 \% Personen aus dem sozialen Umfeld (Sellin/Engels S. 61).

$400 \mathrm{Ob}$ ein Berufsbetreuer auch ehrenamtlich Betreuungen übernehmen kann, bestimmt sich laut Bienwald allein nach der Einstellung der betreffenden natürlichen Person. Zu rechtfertigen sei dies v.a. durch einen Erst-Recht-Schluss aus $₫ 1836$ Abs. 3 und Abs. 1 S. 2, vgl. Bienwald/Sonnenfeld/Hoffmann $\ 1836$ Rn. 5 ff.; ebenso: LG Chemnitz FamRZ 2001, 313; anderer Ansicht ist Zimmermann (Zimmermann FamRZ 1999, 630, 632 und FamRZ 2002, 1373, 1375).

${ }^{401}$ Eine Belastung aus familiären Gründen liegt zumeist dann vor, wenn die betroffene Person bereits mit der Versorgung und Beaufsichtigung minderjähriger Kinder (MünchKommBGB/Schwab \ 1898 Rn. 5) oder pflegebedürftiger Angehöriger betraut ist. Aber auch, wenn Streit mit anderen Angehörigen über die Übernahme der Betreuung besteht (Dodegge/Roth B. Rn. 68; BTDrucks. 11/4528 S. 129). Eine überdurchschnittliche Arbeitsbelastung mit vielen Überstunden oder häufigen beruflich bedingten Ortswechsel kann einen beruflichen Ausschlussgrund darstellen (Dodegge/Roth B. Rn. 68; MünchKommBGB/Schwab \ 1898 Rn. 6). Unter sonstige Verhältnisse fallen hingegen höchstpersönliche Gründe wie das Lebensalter (HK-BUR/Bauer $\ 1898$ Rn. 23; BT-Drucks. 11/4528 S. 129; Dodegge/Roth B. Rn. 68) sowie starke psychische Belastung, große räumliche Entfernung oder eigene Krankheit (BayObLG FamRZ 2005, 931; HKBUR/Bauer $\int 1898$ Rn. 23).

402 BT-Drucks. 11/4528 S. 129; vielmehr sollte die Flexibilität des Gesetzes erhalten bleiben, vgl. Bienwald/Sonnenfeld/Hoffmann $\$ 1898$ Rn. 8. 
übernommen werden kann. ${ }^{403}$ Der Gesetzgeber geht davon aus, dass dies jedem Bürger zugemutet werden kann, außer die damit verbundene persönliche Belastung übersteigt das gewöhnliche Maß erheblich. ${ }^{404}$ Je nach Umfang der Betreuung fällt der Bedarf sehr unterschiedlich aus. Manche Betreute benötigen nur eine geringe, andere eine umfassende Hilfestellung. Das individuelle Maß der Zumutbarkeit richtet sich nach dem Betreuer und nicht nach dem Betroffenen.

Auch ohne ausdrückliche Sanktion in $\int 1898$, ist grundsätzlich jeder Bürger zur Übernahme einer Betreuung verpflichtet. ${ }^{405}$ Dies ergibt sich aus der Systematik des Betreuungsrechts. Es benennt die Betreuung als Ehrenamt ${ }^{406}$ und somit als bürgerliche Pflicht. ${ }^{407}$ Zur Übernahme eines solchen Ehrenamts ist jeder Bürger aus Art. 33 GG verpflichtet. Die Grenze der Zumutbarkeit, ein solches Ehrenamt zu übernehmen, kann durch privatautonome Regelungen und somit durch Wünsche der Betreuungsverfügung nicht ausgestaltet werden. Das Maß der Zumutbarkeit nach $\int 1898$ ist als zwingend anzusehen. Einer individuellen Bestimmung durch die Betreuungsverfügung ist diese Grenze nicht eröffnet.

Im konkreten Fall entsteht die Pflicht nach \ 1898 Abs. 2 erst, wenn der Betroffene sich zur Übernahme bereit erklärt. ${ }^{408}$ Allgemein wird \ 1898 Abs. 2 als Voraussetzung verstanden, die der Betroffene gegenüber dem Betreuungsgericht höchstpersönlich ${ }^{409}$ und ausdrücklich, ${ }^{410}$ positiv erklären muss, ${ }^{411}$ auch wenn der Betreuer vom Betroffenen vorgeschlagen wurde. ${ }^{412}$ Die gesetzlichen Anforderun-

403 Dies ist auch im Hinblick auf die unterschiedliche Belastbarkeit der Menschen sinnvoll (Bienwald/Sonnenfeld/Hoffmann $\$ 1898$ Rn. 8).

404 Dodegge/Roth B. Rn. 68; Jürgens/Jürgens \1898 Rn. 4; juris PK-BGB/Bieg/Jaschinski \ 1898 Rn. 4.

405 Bienwald/Sonnenfeld/Hoffmann \1898 Rn. 1; im Sinne einer Rechtspflicht, vgl. Palandt/-

Diederichsen \1898 Rn. 1: Die Übernahmepflicht der Betreuung eines jeden Bürgers ist nach

\1898 Abs. 1 HS. 2 an seine Eignung und die Zumutbarkeit geknüpft. Bienwald/-

Sonnenfeld/Hoffmann \1898 Rn. 1; zur Parallelvorschrift der Vormundschaft \1785: Staudinger/Engler \1785 Rn. 1; BVerfGE 10, 302, 312; BVerwG NJW 1996, 139, 140; Zimmermann hält die Übernahme der Betreuung ausdrücklich nicht für eine Pflicht, vgl. Soergel/Zimmermann \1786 Rn. 3; Palandt/Diedericbsen \ 1898 Rn. 1.

406 Peters S. 154.

407 Bürgerpflicht, vgl. Bienwald/Sonnenfeld/Hoffmann \1898 Rn. 1 f., 9. Es handelt sich nicht um eine Staatsbürgerpflicht, da die Übernahmepflicht einer rechtlichen Betreuung nicht auf Deutsche beschränkt ist (MünchKommBGB/Schwab \1898 Rn. 2). Rauscher Rn. 1204: Ehrenpflicht.

408 Staudinger/Bienwald \$1898 Rn. 29; Palandt/Diederichsen \$1898 Rn. 1.

409 Bienwald/Sonnenfeld/Hoffmann \1898 Rn. 1.

${ }^{410}$ Soergel/Zimmermann $\$ 1898$ Rn. 6.

411 Dodegge/Roth B. Rn. 70; Damrau/Zimmermann \1898 Rn. 5; Staudinger/Bienwald \1898 Rn. 27. Bei mehreren Betreuern soll die Zustimmung nach \1898 i.V.m. \1899 jedes Einzelnen erforderlich sein (Palandt/Diederichsen \1898 Rn. 5). Bei einem Vereinsbetreuer solle dieser über die Zustimmung des Vereins hinaus nach \$ 1898 i.V.m. \$\$ 1899, 1900 auch selber zustimmen (MünchKommBGB/Schwab \1898 Rn. 9; BayObLG FamRZ 1994, 1061, 1062; Knittel \$ 1898 Rn. 4; Bienwald/Sonnenfeld/Hoffmann \1898 Rn. 4). Auch der einstweilig vorläufig bestellte Betreuer müsse sich zur Übernahme bereit erklären (HK-BUR/Bauer $\$ 1898$ Rn. 27), gleiches gelte für Behördenbetreuer (MünchKommBGB/Schwab \$ 1898 Rn. 9).

412 MünchKommBGB/Schwab \1898 Rn. 9; BayObLG FamRZ 1994, 1061, 1062. 
gen an die Erklärung sind strikt und klar auf die Bereitschaft zur Übernahme der Betreuung ausgerichtet. An eine Bedingung soll sie nicht gebunden werden können, ${ }^{413}$ einer Form nicht unterliegen ${ }^{414}$ und spätestens bis zum Ende des Betreuungsverfahrens eingeholt werden. ${ }^{415}$ Ein Widerruf könne nur bis zum Schluss der Tatsachenverhandlung vorgenommen werden. ${ }^{416}$ Nach der Bestellung zum Betreuer kann der sich zur Übernahme bereit Erklärende nur nach $\int 1908 \mathrm{~b}$ entlassen lassen. ${ }^{417}$ Eine Verweigerung ${ }^{418}$ der Bereiterklärung sieht das Gesetz nicht vor. Allerdings soll eine Bestellung in diesem Fall nicht möglich sein. In diesem Fall wäre nicht gewährleistet, dass die Betreuung ausschließlich im Interesse des Betroffenen erfolge. ${ }^{419}$ Die Bereiterklärung dient vornehmlich dem Zweck, sicherzustellen, dass der ausgewählte zukünftige Betreuer auch tatsächlich zur Übernahme bereit ist. ${ }^{420} \mathrm{Als}$ Voraussetzung für die wirksame Bestellung eines Betreuers ist die

413 Dodegge/Roth B. Rn. 70; anders: Soergel/Zimmermann \1898 Rn. 6 „,hängt von anderen Bedingungen $a b "$ m.w.N.

${ }^{414}$ Dodegge/Roth B. Rn. 70; Damrau/Zimmermann \1898 Rn. 6.

415 MünchKommBGB/Schwab 1898 Rn. 9. Das Fehlen der Bereiterklärung im Rahmen der Betreuerbestellung führt nicht zur Unwirksamkeit sondern zur Anfechtbarkeit der Entscheidung der Betreuerbestellung (Soergel/Zimmermann \$1989 Rn. 8; Dodegge/Roth B. Rn. 70) mit der einfachen Beschwerde (BayObLG FamRZ 1994, 1061; Bassenge/Roth \69g FGG Rn. 18; Damrau/Zimmermann \1898 Rn. 8).

416 MünchKommBGB/Schwab \1898 Rn. 9; Damrau/Zimmermann \1898 Rn. 7; Staudinger/Bienwald \ 1898 Rn. 32.

417 MünchKommBGB/Schwab \1898 Rn. 9; andere Ansicht: LG Duisburg FamRZ 1993, 851; Palandt/Diederichsen \1898 Rn. 5; Dodegge/Roth B. Rn. 70; Damrau/Zimmermann \1898 Rn. 7; Bienwald/Sonnenfeld/Hoffman \$1898 Rn. 15.

418 Diesbezüglich ist zu bedenken, dass die Weigerung nicht ausdrücklich geäußert werden muss. Das Gesetz schreibt hier eine ausdrücklich Übernahmeerklärung vor. Sollte der ausgewählte Betreuer zur Übernahme der konkreten Betreuung nicht bereit sein, genügt das Nichterklären (= Schweigen) seiner Übernahmebereitschaft, vgl. auch Bienwald/Sonnenfeld/Hoffmann \ 1898 Rn. 14.

419 Bienwald/Sonnenfeld/Hoffmann \1898 Rn. 11; BayObLG FamRZ 1994, 1061, 1062; \1898 Abs. 2 enthält laut Bienwald das Konsenssystem des Betreuungsrechts (Bienwald/Sonnenfeld/Hoffmann \ 1897 Rn. 71; Staudinger/Bienwald \ 1898 Rn. 27). Der häufige Wechsel der Vormünder/ Pfleger war ein, durch das Betreuungsrecht zu behebender Kritikpunkt am alten Vormundschaftsrechts (BT-Drucks. 11/4528 S. 50). Aufgrund der bestehenden Pflicht zur Übernahme kann das Moment der Erklärung als verpflichtender Dialog zum Zwecke des Informationsaustauschs in Vorbereitung der Erfüllung der Bürgerpflicht angesehen werden.

${ }^{420}$ Bei dieser Erklärung handelt es sich um eine - vom Gesetzgeber bewusst geschaffene sanktionslose Rechtspflicht (Palandt/Diederichsen \1898 Rn. 1, 5; HK-BUR/Bauer \1898 Rn. 30; LG Duisburg FamRZ 1993, 851). Ursprünglich war ein Zwangsgeld geplant (BT-Drucks. $11 / 4528$ S. 129). Ein solches ist anders als in $₫ 1788$ ausgeschlossen. Dies ist mit Blick auf das zu schützende Vertrauensverhältnis zwischen Betreuer und Betroffenen ausgeschlossen und Folge des Bestrebens einer kontinuierlichen Betreuerbestellung im Interesse des Betreuten. Eine solche ist im Rahmen der Entscheidungen zum Wohl des Betroffenen grundsätzlich zu berücksichtigen (Bienwald/Sonnenfeld/Hoffmann \1908b Rn. 6; MünchKommBGB/Schwab \1908b Rn. 24). Das Vertrauensverhältnis könnte bei einer zwangsweisen Betreuerübernahme nicht entstehen (Palandt/Diederichsen \1898 Rn. 5; HK-BUR/Bauer \1898 Rn. 28; Knittel\$ 1898 Rn. 4; Damrau/Zimmermann \$1898 Rn. 5; Schwab FamRZ 1992, 493, 501). Ein sich weigernder Bürger 
Erklärung als solche mehr als ein formelles Moment bei der Betreuerbestellung. Die Bereiterklärung zur Disposition des Einzelnen frei zu geben, widerspräche dem Sicherungszweck der Norm. Auch wenn in der Regel davon auszugehen ist, dass der Wunschbetreuer zur Übernahme der Betreuung bereit ist, sollte die Erklärung darüber zum Schutz des Betreuten nicht zu seiner Disposition stehen.

\section{Geschäftsfähigkeit und Volljährigkeit des Betreuers}

Das Gesetz nennt die Geschäftsfähigkeit und die Volljährigkeit des Wunschbetreuers nicht als Voraussetzung für seine Bestellung. Nach \1902 ist der Betreuer gesetzlicher Vertreter des Betreuten. ${ }^{421}$ Als solcher vertritt er den Betreuten gerichtlich und außergerichtlich. Für das Handeln als gesetzlicher Vertreter gelten die $\iint 164$ ff. ${ }^{422}$ Der Umfang der Vertretungsmacht richtet sich dabei nach dem Umfang der Aufgabenkreise. ${ }^{423}$ Der Betreuer soll geeignet sein, die Angelegenheiten des Betreuten rechtlich zu besorgen. ${ }^{424}$ Das Gesetz sieht Geschäftsunfähige i.S.d. SS 104, 105 generell als unfähig an, am rechtsgeschäftlichen Verkehr teilzunehmen und Willenserklärungen - egal ob für sich oder einen anderen ${ }^{425}$ - wirksam abzugeben.

Der Minderjährige, der das 7. Lebensjahr vollendet hat, ist nach den Maßgaben der $\iint 107$ ff. beschränkt geschäftsfähig. Soweit dies zur Abwendung einer Gefahr für die Person oder das Vermögen eines unter Betreuung stehenden Erwachsenen erforderlich ist, ordnet das Betreuungsgericht an, dass der Betreuer zu einer Willenserklärung, die den Aufgabenkreis des Betreuten betrifft, die Einwilligung des Betreuten bedarf, vgl. \1903. Weil ein rechtlicher Vertreter nur dann für und gegen den Betreuten entscheiden und rechtswirksam handeln kann, wenn er wirksam Willenserklärungen empfangen und abgeben kann, muss er geschäftsfähig ${ }^{426}$ sein.

Das Familienrecht regelt in $\int 1673$ Abs. 1 und 2 ausdrücklich, dass die elterliche Sorge ${ }^{427}$ ruht, wenn ein Elternteil geschäftsunfähig oder in der Geschäftsfähigkeit beschränkt ist. Nach $\iint 1780,1781$ Nr. 1 kann ein Geschäftsunfähiger nicht, ein beschränkt Geschäftsfähiger soll nicht zum Vormund bestellt werden.

soll laut Gesetzbegründung nicht zum Betreuer bestellt werden können, ,da nicht zu erwarten ist, dass der Betreffende seine Pflichten erfüllen wird", BT-Drucks. 11/4528 S. 129. Jedoch kann gegen ihn gem. JS 1787 Abs. 1, 1908 i Abs. 1 S. 1 ein Schadensersatzanspruch geltend gemacht werden, wenn aufgrund seiner Weigerung dem Betroffenen ein Schaden entstanden ist (Palandt/-

Diederichsen S 1898 Rn. 1; HK-BUR/Bauer S 1898 Rn. 30; Knittel S 1898 Rn. 5).

421 Bienwald/Sonnenfeld/Hoffmann S 1902 Rn. 1; Neubausen RNotZ 2003, 157, 165.

422 Neubausen RNotZ 2003, 157, 165.

423 Neubausen RNotZ 2003, 157, 166.

424 Bamberger/Roth/Müller $\$ 1897$ Rn. 7.

425 Larenz/Wolf 546 Rn. 26.

426 BT-Drucks. 11/4528 S. 125; Palandt/Diederichsen S 1897 Rn. 5; Bamberger/Roth/Müller

\ 1897 Rn. 7; MünchKommBGB/Schwab \1908b Rn. 6.

${ }_{427}$ Und in der Folge die damit einhergehende gesetzliche Vertretungsmacht nach $\int 1629$ Abs. 1 S. 1. 
Die Aufgaben des Vormundes als Rechtsfürsorge für Person und Vermögen des Mündels (vgl. \$ 1793) und die Besorgung der rechtlichen Angelegenheiten des Betreuten (vgl. \1901 Abs. 1) sind vergleichbar. Die Entwicklung des Betreuungsrechts aus dem Vormundschaftsrecht erlaubt grundsätzlich die Übernahme dieser Regelung für das Betreuungsrecht. ${ }^{428}$ Ein Verweis in $\$ 1908 \mathrm{i}$ auf $\ 1780$ fehlt. Auf ihn hat der Gesetzgeber bewusst verzichtet. ${ }^{429}$ Als ,,unnötig“ wurde die Einführung einer Parallelvorschrift zu $\ 1780$ eingestuft. „Der Betrener ist gesetzlicher Vertreter des Betreuten; die mangelnde Eignung eines Geschäftsunfähigen oder beschränkt Geschäftsfäbigen zum Betreuer ist offensichtlich. " 430 Die alten Regelungen des geltenden Rechts enthielten nach der Gesetzesbegründung zum Betreuungsrecht teilweise „unnötige Selbstverständlicbkeiten" und seien ,für ein modernes Betrenungsrecht zu kasuistisch" und zu starr. ${ }^{431}$ In den $\int \$ 1897$ ff. wurde aus diesem Grund auf eine Regelung zur Geschäftsfähigkeit des Betreuers verzichtet. Dem Sinn nach ist den $\int \mathbb{S} 1780,1781$ wegen $\ 104$ Nr. 2 und $\ 105$ Abs. 1 für den Geschäftsunfähigen und wegen \1673 Abs. 2 HS 2 für den beschränkt Geschäftsfähigen auch für das Betreuungsrecht Geltung zuzusprechen. ${ }^{432}$ Ein beschränkt Geschäftsfähiger kann daher nicht zum Betreuer bestellt werden. Gleiches gilt wegen \1903 Abs. 1 S. 2 für den unter Einwilligungsvorbehalt Stehenden für den betroffenen Aufgabenbereich. Weil der Gegenbetreuer nicht gesetzlicher Vertreter des Betreuten ist, ${ }^{433}$ gelten für diesen nicht zwingend dieselben Maßstäbe. Die Eignung fehlt ihm aber ebenfalls.

Die im Rahmen einer Betreuungsverfügung getroffene anderweitige privatautonome Regelung kann keinen Vorrang vor diesen Grundsätzen des Betreuungsrechts haben. Ein Vorrang würde die Funktion des Betreuungsrechts umgehen. Die Voraussetzungen Geschäftsfähigkeit und Volljährigkeit des Betreuers können mit einer Betreuungsverfügung nicht abgedungen werden.

VII. Keine eigene Betreuungsbedürftigkeit des Betreuers

Das Betreuungsrecht enthält keine Regelung zur Frage, ob der Betreuer selber unter Betreuung stehen darf. Das Vormundschaftsrecht hat dies in $\$ 1781 \mathrm{Nr} .2$ ausdrücklich geregelt. Es hält denjenigen als zur Übernahme der Vormundschaft für untauglich, für den ein rechtlicher Betreuer bestellt ist. Nach dem Wortlaut soll eine betreute Person als Vormund nicht bestellt werden. Auf das Betreuungsrecht ist dieser Grundsatz zu übertragen. ${ }^{434}$ Es ist dahingehend zu modifizieren, dass der Betreffende zumindest dann nicht zum Betreuer bestellt werden kann, wenn

\footnotetext{
${ }^{428}$ So Soergel/Zimmermann \1897 Rn. 4 mit Blick auf \} 1 7 8 1 .

${ }^{429}$ Vgl. BT-Drucks. 11/4528 S. 125.

430 BT-Drucks. 11/4528 S. 125

431 BT-Drucks. 11/4528 S. 125.

432 MünchKommBGB/Schwab \1908b Rn. 6; Staudinger/Bienwald \$ 1897 Rn. 20; ähnlich: Palandt/-

Diederichsen $\ 1897$ Rn. 5; keine sinngemäße Anwendung der \$S 1780, 1781 nach Meinung von

Bienwald/Sonnenfeld/Hoffmann \1897 Rn. 43.

433 Bienwald/Sonnefeld/Hoffmann \$1902 Rn. 1.

${ }^{434}$ Staudinger/Bienwald \ 1897 Rn. 22; Schwab FamRZ 1992, 493, 501; Vgl. \ 3 B. VI.
} 
er selber im gleichen Aufgabenbereich betreut wird. ${ }^{435}$ Ist er selber in diesen Fragen aufgrund einer psychischen Krankheit, einer körperlichen, geistigen oder seelischen Behinderung nicht in der Lage, seine Angelegenheiten ganz oder teilweise zu erledigen, dann kann er erst recht nicht die eines anderen erledigen. Die Möglichkeit der privatautonomen Disposition dieses Grundsatzes würde die Funktion des Betreuungsrechts als rechtliche Hilfe aufheben. Die Nennung eines Wunschbetreuers in einer Betreuungsverfügung, der selber für den fraglichen Aufgabenbereich unter Betreuung steht, entfaltet daher keine Wirkung.

\section{Zusammenfassung}

Zusammenfassend ist festzustellen, dass der Betroffenen auf die Eignung der Person des Betreuers, die notwendige Voraussetzung seiner Volljährigkeit und den Grad seiner Geschäftsfähigkeit, die Einschätzung der Zumutbarkeit der Übernahme einer Betreuung, dem Ausschluss einer Person, die in der Anstalt, dem Heim oder einer sonstigen Einrichtung nach \ 1997 Abs. 3 beschäftigt ist, in der der zu Betreuende untergebracht ist, sowie auf den Vorrang der privaten, auch berufsmäßig geführten Einzelbetreuung vor der Betreuung durch einen Verein oder eine Behörde und dem Vorrang der ehrenamtlichen vor der beruflich geführten Betreuung nicht mittels eigener rechtlich anerkannter Wünsche in Form der Betreuungsverfügung Einfluss nehmen kann. Diese Voraussetzungen sind zwingend und können nicht durch den Betroffenenwunsch abgedungen werden.

\section{Ergebnis zu $\$ 3$}

Die Untersuchung zeigt, dass der Betroffene mittels einer Betreuungsverfügung auf die Auswahl des Betreuers unter Beachtung bestimmter gesetzlicher Vorgaben Einfluss nehmen kann. An seine positiven oder negativen Vorschläge bzgl. der Person des Betreuers ist das Betreuungsgericht gebunden, soweit sie nicht gegen eine Betreuung insgesamt gerichtet sind.

Der von ihm benannte Wunschbetreuer muss zur Übernahme der Einzelbetreuung darüber hinaus geeignet und bereit sein und darf in keiner in \1897 Abs. 3 genannten Verbindung zur Einrichtung oder dem Heim stehen, in dem der Betroffenen lebt. Zudem muss er voll geschäftsfähig sein, darf im einschlägigen Aufgabenkreis nicht selber unter Betreuung stehen und die Übernahme der Betreuung darf ihm nicht unzumutbar sein. Diese Voraussetzungen sind zwingend und beantworten die Frage nach dem „Ob“ der Wunscherfüllung des Betroffenen.

\footnotetext{
435 Bamberger/Roth/Müller \ 1897 Rn. 7; MünchKommBGB/Schwab \1908b Rn. 6; Palandt/Diederichsen \ 1897 Rn. 5; Bienwald schließt eine Bestellung dann nicht grundsätzlich aus (Staudinger/Bienwald S 1897 Rn. 22).
} 



\section{\4 Einfluss der Betreuungsverfügung auf das Verfahren der Betreuerbestellung}

Das Betreuungsverfahren beginnt weder zu einem vorher festgelegten Zeitpunkt ${ }^{436}$ noch mit einer öffentlichen Bekanntmachung ${ }^{437}$. Damit nicht jeder Antrag zur Einleitung eines Verfahrens nach $\$ \int 26$ ff. FamFG/ $\int \mathbb{S} 12$ ff. FGG oder $\iint 278$ FamFG/ $\iint 68$ ff. FGG führen muss, wird teilweise eine Vorermittlung zur Zusammentragung wesentlicher Unterlagen und der Kontaktaufnahme zu Behörden zur Ermittlung möglicher dort vorhandener Erkenntnisse befürwortet. 438 Bereits in diesem Stadium - also vor Aufnahme von Ermittlungen - soll nach vereinzelter Meinung dem Betroffenen Gelegenheit zur Äußerung gegeben werden. Dies sei Ausdruck der weitmöglichsten Beachtung des Betroffeneninteresses $^{439}$ und spiegele die Ziele des Betreuungsrechts mit der Anerkennung der Subjektivität jeder beteiligten Person. ${ }^{440} \ 275$ FamFG (\$ 66 FGG) regelt, dass der Betroffene in Betreuungssachen ohne Rücksicht auf seine Geschäftsfähigkeit ver-

436 Bienwald/Sonnenfeld/Hoffmann Vorbem. v. SS 65 ff. FGG Rn. 16.

437 BT-Drucks. 11/4528 S. 89.

438 Bienwald/Sonnenfeld/Hoffmann Vorbem. v. $\iint 65$ ff. FGG Rn. 16.

439 Jürgens/Kröger/Marschner/Winterstein Rn. 298.

440 BT-Drucks. 11/4528 S. 52; MünchKommZPO/Schmidt-Recla \275 FamFG Rn. 4 und ausführlich oben \$2 C. II.: V.a. Beachtung des Betroffenenwohls, seine persönliche Betreuung und die Stärkung der Personensorge. 
fahrensfähig ist. Damit verfolgt der Gesetzgeber das Ziel, dass Anträge, Wünsche und Vorschläge des Betroffenen verbindlich sein sollen, soweit dies verantwortet werden könne. ${ }^{441}$ Dies gilt auch für das Verfahren, an dessen Ende bei Erforderlichkeit die Einrichtung der Betreuung steht.

$\mathrm{Zu}$ untersuchen ist, inwieweit der Betroffene mittels Wünschen und Vorstellungen der Betreuungsverfügung bereits im Vorhinein für den eventuellen Fall eines Verfahrens auf dieses individuell einwirken kann. Konkret stellt sich die Frage, ob und inwieweit eine privatautonome Einflussnahme bei der Bestellung, der Auswahl und der Vergütung des Verfahrenspflegers und des Sachverständigen, der Anwesenheit und Anhörung Dritter und auf das Handeln des Gerichts bei Gefahr in Verzug möglich ist. Fraglich ist auch, ob der Betroffene mittels Wünschen sogar den Umfang seiner Betreuung bestimmen, also festlegen kann, für welche Angelegenheiten eine Betreuung eingerichtet werden soll.

Unterliegt das Verfahren gesetzlich zwingenden Regelungen, sind allein diese maßgeblich. Sind die Regelungen dispositiv, ist die Einfluss des Betroffenen mittels in der Betreuungsverfügung antizipiert geäußerter Wünsche möglich. Wie und in welchem Umfang ist im Folgenden zu prüfen. Sind die Regelungen disponibel, gilt der allgemeine Wunschbefolgungsgrundsatz des \ 1901. Seinem Wortlaut nach ist er zwar auf die Führung der Betreuung beschränkt. Es entspricht aber den Zielen des Betreuungsrechts, die Wünsche und Interessen des Betroffenen gem. $\int 1901$ auch im Verfahren umfassend Geltung zukommen zu lassen. Auch spiegelt dies das verfassungsrechtlich begründete Prinzip, wonach der Wille des Betroffenen grundsätzlich zu beachten ist und seine Nichtbeachtung gerechtfertigt werden muss. ${ }^{442}$

\section{A. Umfang der Betreuung}

Kann der Einzelne den Umfang seiner - hypothetisch notwendigen - Betreuung nach seinen eigenen Wünschen und Vorstellungen bestimmen, würde er den Umfang seiner Betreuung antizipiert mit Hilfe der Betreuungsverfügung festlegen. „Ob“ und wenn ja, „Wie“ ein solch individueller Einfluss des Einzelnen möglich ist, ist v.a. anhand von $\ 1901 \mathrm{zu}$ untersuchen.

I. Bestimmung der Aufgabenkreise gem. \1901 Abs. 1

Nach \1901 Abs. 1 umfasst die Betreuung, alle Tätigkeiten, die erforderlich sind, um die Angelegenheiten des Betreuten [...] rechtlich zu besorgen". Welche diese vom Betreuer zu besorgende Angelegenheiten sind, bestimmt sich nach dem jeweiligen Aufgabenkreis. Dieser wird wiederum gem. \1896 Abs. 2 S. 1 nur für den Bereich bestimmt, in dem eine Betreuung erforderlich ist. Der Umfang der Erforderlichkeit

441 BT-Drucks. 11/4528 S. 52.

$442 \mathrm{Vgl}$. die Ausführungen in $\$ 2$ A. 
wird bei Einrichtung der Betreuung durch das Betreuungsgericht nach $\ 286$ Abs. 1 Nr. 1 FamFG ( $(69$ Abs. 1 Nr. 2b FGG) in Form von Aufgabenkreisen bestimmt und während der Betreuung gem. \293 Abs. 1 FamFG (\$ 69i Abs. 1 FGG) nach Bedarf in einem regulären Verfahren angeglichen. Das vor der Betreuerbestellung einzuholende Sachverständigengutachten hat sich nach $\ 280$ Abs. 3. Nr. 4 FamFG ( $(68$ b Abs. 1 S. 5 FGG) auf die Frage der Aufgabenkreise zu erstrecken und anzuzeigen, in welchem Umfang dem Betroffenen Fähigkeiten geblieben sind, seine Angelegenheiten selbst zu besorgen. ${ }^{443}$ In $57 \%$ aller Betreuungsfälle bleiben während der Betreuung die Aufgabenkreise bestehen, die bei Erstbestellung der Betreuer vergeben wurden. ${ }^{444}$ Sollte der Einzelne mittels der Betreuungsverfügung auf den Umfang seiner Betreuung Einfluss haben, müsste der Grundsatz der Erforderlichkeit ihm dies erlauben.

Die Übertragung aller Aufgaben ist im materiellen Recht nicht beschrieben. Das Verfahrensrecht hält dies nach $₫ 276$ Abs. 1 Nr. 2 FamFG (\$ 67 Abs. 1 Nr. 2 FGG ${ }^{445}$ für möglich. Gesetzlich bestimmt sind nur einige Aufgaben: \ 1896 Abs. 3 nennt die Geltendmachung von Rechten des Betroffenen gegenüber seinem Bevollmächtigten, Abs. 4 Entscheidungen über den Fernmeldeverkehr des Betroffenen und über die Entgegennahme, das Öffnen und das Aufhalten von Post. \1899 Abs. 2 nennt die Entscheidung über die Einwilligung in die Sterilisation des Betreuten. Die Aufzählung zeigt, dass es sich hier um eine beispielhafte und nicht abschließende handelt. ${ }^{446}$ Durch eine fehlende gesetzliche Definition sollte die frühere Praxis der pauschalen und umfassenden Bestimmung der Wirkungskreise bei der Gebrechlichkeitspflegschaft beendet werden und einer strengen Erforderlichkeitsprüfung weichen. ${ }^{447}$ Der Gesetzgeber vermied bewusst eine Typisierung der Aufgabenkreise, um die Möglichkeit einer differenzierten Bestimmung zu gewährleisten. ${ }^{448}$ Dies soll der Grundsatz der Erforderlichkeit sicherstellen.

\section{Typisierung der Aufgabenkreise nach Rechtsprechung und Literatur}

Der ursprünglich nur aus den heutigen Abs. 2 - 5 bestehende $\ 1901$ wurde nachträglich um Abs. 1 ergänzt. ${ }^{499}$ Dies war die gesetzgeberische Reaktion auf die in Rechtsprechung und Literatur ${ }^{450}$ unterschiedliche Umschreibung der Aufgabe des

\footnotetext{
443 Palandt/Diederichsen \1896 Rn. 15; Staudinger/Bienwald \$ 1896 Rn. 151.

444 Sellin/Engels S. 80.

445 Damrau/Zimmermann \67 FGG Rn. 27.

446 BT-Drucks. 11/4528 S. 121.

447 BT-Drucks. $11 / 4528$ S. $120 \mathrm{f}$.

448 BT-Drucks. 11/4528 S. 121.

449 Änderung durch Art. 1 Nr. 1, 10a, 12, 13 BtÄndG; zudem wurden Überschriften von den

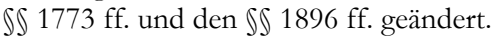

450 Siehe zur Diskussion: BT-Drucks. 13/7158 S. 33 f.; auch jetzt ist die Auslegung unterschiedlich, enges Verständnis, vgl. MünchKommBGB/Schwab \1897 Rn. 40, weites Verständnis, vgl. Knittel \1897 Rn. 1 und Soergel/Zimmermann \1896 Rn. 18.
} 
Betreuers. Zum Teil wurde diese ausschließlich als tatsächliche, im Sinne einer pflegerischen, verstanden. ${ }^{451}$ V.a. während der ersten Anwendung des Betreuungsrechts wurden die Aufgabenkreise teilweise von den Gerichten sehr unterschiedlich benannt und zudem inhaltlich verschieden definiert. ${ }^{452}$ Teilweise knüpften die Richter an die Wirkungsbereiche der Pflegschaft an. ${ }^{453}$ Mittlerweile hat sich die Rechtsprechung etwas vereinheitlicht. In einigen Bereichen wurden typisierte Aufgabenkreise gebildet. ${ }^{454}$ Die bedeutendsten Aufgabenkreise Vermögenssorge, Gesundheitssorge und Aufenthaltsbestimmung bestimmen die Gerichte unterschiedlich: zum Teil fein untergliedert, ${ }^{455}$ teilweise ohne weitere Aufgliederung. ${ }^{456}$ Im Übrigen werden die Aufgabenkreise jedoch einzelfallbezogen durch die Gerichte beschrieben. ${ }^{457}$

Durch Abs. 1 wird nunmehr die Beschreibung der Betreuertätigkeit einheitlich als Rechtsfürsorge angenommen. ${ }^{458}$ Dies hatte der Gesetzgeber zwar bereits in der Gesetzbegründung zum Betreuungsgesetz klargestellt, ${ }^{459}$ es fand in der Praxis vor Einführung des jetzigen Abs. 1 jedoch nur zum Teil Beachtung. Die Rechtsprechung knüpft bei der Bestimmung der Aufgabenkreise mittlerweile einheitlich an das Erforderlichkeitsprinzip. ${ }^{460}$ Danach sind solche Angelegenheiten nicht erfasst, die der Betroffene noch eigenständig erledigen 461 bzw. der mit der Betreuung angestrebte Zweck nicht mit weniger eingreifenden Mitteln erreicht werden kann. ${ }^{462}$ Die Erforderlichkeit der Betreuung muss für jede Aufgabe konkretisiert werden. 463

In der Praxis wird in 6,8 \% der Fälle 464 nur eine Angelegenheit auf den Betreuer übertragen. Die Übertragung aller Angelegenheiten nach \ 286 Abs. 1 Nr. 1 FamFG (\69 Abs. 1 Nr. 2b FGG), \13 Nr. 2 BWahlG, die die Ausnahme bleiben

\footnotetext{
451 Vgl. nur Staudinger/Bienwald \ 1901 Rn. 2, 17 ff.; BT-Drucks. 13/7158 S. 33; Bienwald/Sonnenfeld/Hoffmann \ 1901 Rn. 1.

452 Vgl. die Auflistungen bei Bienwald BtG, 3. Aufl. \ 1896 Rn. 214 ggü. Bienwald/Sonnenfeld/-

Hoffmann $\int 1896$ Rn. 133.

453 Vgl. zum Pflegschafts- und Vormundschaftsrechts Zenz/Eicken/Ernst/Hofmann S. 13.

454 Auch aufgrund der Arbeit durch die Literatur, siehe nur Bienwald BtG, 3. Aufl. \1896 Rn. 214 ff. und HK-BUR/Bauer \$1896 Rn. 221.

455 OLG Zweibrücken FamRZ 2000, 1324.

456 Vgl. nur die Ausführungen bei HK-BUR/Bauer \1896 Rn. 224 ff.

457 Siehe hierzu den Überblick bei Palandt/Diederichsen \$ 1896 Rn. 20.

458 BT-Drucks. 13/7158 S. 33; Soergel/Zimmermann S 1901 Rn. 3.

${ }^{459} \mathrm{Vgl}$. BT-Drucks. 11/4528 S. 122.

460 BayObLG FamRZ 1994, 1552; BayObLG FamRZ 1995, 1085; BayObLG BtPrax 2001, 79.

461 BayObLG BtPrax 2001, 79.

462 BayObLG BtPrax 1994, 209.

463 BayObLG FamRZ 1994, 1552; BayObLG FamRZ 1995, 116; BayObLG FamRZ 1995, 1085; OLG Köln FamRZ 1996, 249; BayObLG FamRZ 1996, 897; BayObLG BtPrax 1997, 72, 73; BayObLG FamRZ 1997, 902, 903; BayObLG FamRZ 1998, 920; BayObLG FamRZ 1998, 921; BayObLG FamRZ 1999, 1612, 1613; OLG München FamRZ 2006, 575.

464 Sellin/Engels S. 79 - ohne die Bestellung für ,alle“ Angelegenheiten.
} 
soll, 465 erfolgt in $7 \%$ der Betreuungen, wobei dies regional sehr unterschiedlich ausfällt. 466

Die Literatur hat sich an einer Typisierung der Aufgabenkreise beteiligt. ${ }^{467}$ Anlässlich von Fragen, die die Sorge der Gesundheit des Betreuten betreffen, ist aufgefallen, dass die Legitimation des Betreuers für seine Entscheidungen in diesem Bereich unterschiedlich definiert wird. Nach einer Ansicht enthält die vormundschaftlich vorgenommene Zuweisung eines Sachgebietes an den Betreuer zugleich die Befugnis, die zur Durchführung der konkreten Aufgabe dieser Betreuung nötig erscheint. ${ }^{468}$ Nach anderer Auffassung muss die konkrete Befugnis in dem Bestellungsbeschluss des Betreuers ausdrücklich verlautbart sein. ${ }^{469}$ Der dieser Diskussion zugrunde liegende Streit über die Befugnis zur Einrichtung und die Grenzen der Betreuung und der damit verbundenen Fremdbestimmung betrifft auch die Frage, wie vorausschauend eine Betreuung einzurichten ist. Einerseits wird vertreten, der Aufgabenkreis solle so groß sein, dass ein absehbarer Betreuungsbedarf einzubeziehen sei, damit nicht fortlaufend Erweiterungen des Aufgabenkreises und damit immer wieder neue, den kranken Menschen belastende Gerichtsverfahren veranlasst werden müssten. ${ }^{470}$ Die Mehrheit hält aufgrund des durch die Einrichtung der Betreuung erfolgenden Grundrechtseingriffs nur den im Zeitpunkt der Einrichtung konkret erforderlichen Bedarf für maßgeblich. ${ }^{471}$ Das Recht auf Selbstbestimmung soll nicht weiter eingeschränkt werden, als zum Schutz des Betroffenen nötig. ${ }^{472}$

\section{Zwischenergebnis}

Die Bestellung einer Betreuung nach $\iint 1896$ ff. ist ein Eingriff in das Selbstbestimmungsrecht des Betroffenen. Als Grundrechtseingriff bedarf dieser der Rechtfertigung und muss so gering wie möglich gehalten werden. Nach der Konzeption des Gesetzgebers ist der Eingriff in das Recht auf Selbstbestimmung gerechtfertigt, wenn die Betreuerbestellung erforderlich ist. Nach dem \1896 Abs. 1 und 2 zugrunde liegenden Erforderlichkeitsprinzip ${ }^{473}$ ist eine Betreuung

\footnotetext{
465 Jürgens/Jürgens \1896 Rn. 29; Bienwald Verfahrenspflegschaftsrecht Rn. 72 f.; MünchKommBGB/Schwab \$1896 Rn. 107.

466 Sellin/Engels S. 80.

467 Überblick bei Bienwald BtG, 3. Aufl. \1896 Rn. 212 ff.; Harm Rpfleger 1998, 89 ff.

468 MünchKommBGB/Schwab \1896 Rn. 65; Soergel/Zimmermann \1896 Rn. 43 f.

${ }^{469}$ Lipp S. 98 ff., 242; so auch die Rechtsprechung, vgl. nur OLG München BtPrax 2006, 30 ff.; BayObLG FamRZ 1995, 116; OLG Hamm FamRZ 1995, 433, 435; BayObLG FamRZ 1995, 116.

470 Schwab FamRZ 1992, 493, 495; Ebenso im Ansatz: Pankoke-Schenk NDV 1989, 49, 51; Zimmermann fordert eine vorausschauende Betrachtung in den typischen Erweiterungsfällen (Soergel/Zimmermann $\$ 1896$ Rn. 61).

471 Palandt/Diederichsen \1896 Rn. 8-10, 19; Staudinger/Bienwald \1896 Rn. 55; Peters S. 81; Schwab K 28; HK-BUR/Bauer 1896 Rn. 217; Zen₹/Eicken/Ernst/Hofmann S. 47; BayObLG BtPrax 1995, 218, 219; BayObLG NJW-RR 1998, 158 f.; OLG München BtPrax 2006, 30 ff. 472 Scbwab K 27; HK-BUR/Bauer \1896 Rn. 217.
} 
und 2 zugrunde liegenden Erforderlichkeitsprinzip 473 ist eine Betreuung nur möglich, wenn der Betroffene aufgrund einer Krankheit in einem bestimmten Bereich nicht mehr die Fähigkeiten besitzt, seine Angelegenheiten allein zu erledigen. ${ }^{474}$ Zudem muss eine Betreuungsbedürftigkeit bestehen. ${ }^{475}$ Die Angelegenheiten dürfen also nicht von einem Bevollmächtigten oder durch andere Hilfen ebenso gut wie durch einen Betreuer besorgt werden können. Dies muss konkret in diesem Zeitpunkt $t^{476}$ vorliegen. Worin konkret Hilfe erforderlich ist, bestimmt sich an der individuellen, krankheitsbedingten Hilflosigkeit und der fehlenden Alternative zur Betreuung. Welche Aufgabenkreise in einer Betreuung auf den Betreuer zu übertragen sind, stellt das Gericht unter Berücksichtigung der konkreten Lebenssituation des Betroffenen fest. ${ }^{477}$ Demnach sind nur solche Aufgaben zu übertragen, die nach seiner Stellung und seiner bisherigen Lebensführung im Interesse des Betroffenen wahrgenommen werden müssen. ${ }^{478}$ Die Übertragung einer Aufgabe aufgrund des Wunsches des Betreuten würde dies ändern. Dann wäre für die Übertragung der einzelnen Aufgabe nicht maßgebend, ob der zu Betreuende zum einen in dieser Sache gem. \1896 Abs. 1 S. 1 aufgrund einer krankhaften Veränderung nicht mehr in der Lage ist, sie zu erledigen und ihre rechtliche Besorgung zum anderen nicht privatautonom auf einen Dritten übertragen hat. Maßstab der Betreuungseinrichtung wäre damit nicht mehr der Erforderlichkeitsgrundsatz nach \ 1896 Abs. 1 S. 1 und Abs. 2 S. 2. Die Einführung der Erforderlichkeitsprüfung zum Schutz des Betreuten vor ungerechtfertigten Eingriffen gilt als Teil der Verhältnismäßigkeitsprüfung und hat somit Verfassungsrang. ${ }^{479}$ Nach der gesetzlichen Begründung soll er dem Schutz der Betroffenen vor ungerechtfertigten Maßnahmen und dem öffentlichen Interesse dienen. Wobei letzteres der entscheidende Gesichtspunkt darstellt, weil er dem Eingriff zustimmen kann. Auf seine Beachtung soll der Betroffene daher nicht verzichten können. ${ }^{480}$

Nach der gesetzlichen Konzeption soll auch im Verfahren der Betreuerbestellung der Wunsch des Betreuten Entscheidungs- und Handlungsmaßstab für das Betreuungsgericht sein. ${ }^{481}$ Die Wunschbefolgungspflicht des $₫ 1901$ Abs. 3 erlaubt

\footnotetext{
473 Dieses wird zudem genannt in $\iint 1903,1906$ Abs. 1, 1908d Abs. 3.

${ }^{474}$ Dies wird zum Teil auch mit „Betreuungsbedürftigkeit“ beschrieben, vgl. etwa Palandt/Diederichsen $\$ 1896$ Rn. 8.

475 Zum Teil wird alternativ auch von subjektiver Betreuungsbedürftigkeit und objektivem Betreuungsbedarf gesprochen, vgl. Schwab FamRZ 1992, 493, 495.

476 Staudinger/Bienwald \$1896 Rn. 43; Bienwald BtG, 3. Aufl. \1896 Rn. 76 ff.

477 Dodegge/Roth A. Rn. 21, 22; Jürgens/Kröger/Marschner/Winterstein Rn. 82, 83; BT-Drucks. $11 / 4528$ S. $120 \mathrm{f}$.

478 Jürgens/Kröger/Marschner/Winterstein Rn. 83; Jürgens/Jürgens $₫ 1896$ Rn. 9.

479 BT-Drucks. 11/4528 S. 120; BVerfGE 19, 342, 348 f.; BVerfGE 58, 208, 225 f.; BVerfG NJW 1994, 1577, 1578 f.; MünchKommBGB/Schwab \$ 1896 Rn. 24; Sie eignet sich nach Gessaphe aber nicht zur Feststellung der Notwendigkeit der Betreuung, vgl. S. 222-227.

480 BT-Drucks. 11/4528 S. 121.

481 Nach der Gesetzesbegründung sollen Äußerungen des Betreuten in besonderem Maße anerkannt werden (BT-Drucks. 11/4528 S. 68).
} 
dem Betroffenen durch eigene Wünsche und Vorstellungen sein Wohl und mit diesem eine Grenze für die Bindung seiner Wünsche zu formulieren. ${ }^{482}$ Folglich gewährt das Gesetz in \1901 einen Einfluss des Betreuten auf die Maßstäbe zur Umsetzung seiner Betreuung. Daraus lässt sich hingegen kein ausdrücklicher Einfluss auf den Umfang seiner Betreuung herleiten.

Gem. \1836 Abs. 1, $\int 1$ des Gesetzes über die Vergütung von Vormündern und Betreuern (VBVG) wird die Betreuung grundsätzlich ehrenamtlich und somit unentgeltlich geführt. ${ }^{483}$ Bei einer berufsmäßigen Betreuung hat der Betreute hingegen den Berufsbetreuer gem. \1836 zu vergüten. Die Zahl der Berufsbetreuungen bei mittellosen Betreuten steigt. In diesem Fall kann der Berufsbetreuer seinen Anspruch auf Vergütung nach $\int 1$ Abs. 2 S. 2 VBVG dem Staat gegenüber geltend machen. Der individuelle Einfluss auf den Umfang der Betreuung würde daher in vielen Fällen Mehrkosten für die Staatskasse bedeuten. Dies würde wiederum nicht dem Grundsatz der Erforderlichkeit entsprechen, weil dieser auch öffentlichen Interessen, d.h. auch der Schonung der Staatskasse, dient. ${ }^{484}$

Auch nach Rechtsprechung und Literatur reicht der bloße Wunsch des Betroffenen nach Betreuung zur Einrichtung der Betreuung nicht aus. ${ }^{485}$ Die Einrichtung einer Betreuung lediglich aufgrund des Wunsches des Betreuten würde den für alle vergleichbaren Maßstab der Erforderlichkeit vereiteln. Dadurch würden aufgrund des individuellen Wunsches begrenzte staatliche Leistungen wie Gerichte, Berufsbetreuer und Behörden von dem Betroffenen in Anspruch genommen und ggf. aufgebraucht werden, obwohl ihm ggf. Alternativen zur Verfügung stehen, die in einem Sozialstaat für alle Bedürftigen zur Verfügung stehen sollen.

Die gesetzlichen Formulierungen erlauben an keiner Stelle eine konkrete Einflussnahme durch den Betroffenen auf den Umfang der Betreuung mittels der Betreuungsverfügung. Vielmehr fordert \1896 Abs. 2 S. 1 ausdrücklich die Untersuchung und positive Feststellung der Erforderlichkeit der Betreuerbestellung. Erforderlich kann eine Betreuung nur dann sein, wenn der Betroffene seine Angelegenheiten aufgrund einer Krankheit nicht mehr besorgen kann und weder der Betroffene selbst, noch andere Personen die Aufgabenbereiche, für die Handlungsbedarf besteht, wahrnehmen können. ${ }^{486}$ Der Auftrag an den Betreuer (Aufgabenkreis) darf dabei nicht mehr beinhalten, als der Betroffene zu besorgen hat ${ }^{487}$ und muss für jeden Aufgabenbereich konkretisiert werden. ${ }^{488}$ Der Gesetzgeber hat sich zwar für die Mitwirkung des Einzelnen bei der Ausgestaltung des Verhältnis-

\footnotetext{
482 Vgl. oben $\int 2$ C. III.

483 Vgl. die Ausführungen unter $₫ 5 \mathrm{~B}$.

484 Soergel/Zimmermann \$1896 Rn. 39; Müller/Renner Rn. 50.

485 OLG Köln FamRZ 1996, 249, 250; Müller/Renner Rn. 50; Soergel/Zimmermann \ 1896 Rn. 39.

486 Staudinger/Bienwald $\int 1896$ Rn. 51.

487 Geringst möglicher Umfang Staudinger/Bienwald \1896 Rn. 47; Jürgens/Kröger/Marschner/Winterstein Rn. 78.

488 Staudinger/Bienwald \$1896 Rn. 51.
} 
ses der Betreuung entschieden ${ }^{489}$ und erlaubt diesen Einfluss, wenn er dem wirklichen Willen des Betroffenen entspricht, und dieser daran noch festhalten will. ${ }^{490}$ Nach der gesetzlichen Formulierung, dem Sinn und Zweck und der Gesetzesbegründung bezieht sich dies jedoch nur auf das „Wie“ einer Betreuung. In der Literatur fordern selbst die in dieser Frage „Liberalen“ zumindest die Zuweisung des Sachgebietes durch das Gericht. ${ }^{491}$ Im konkreten Fall erlaubt \ 1901 dem Einzelnen durch das Formulieren seiner konkreten Wünsche und allgemeinen Vorstellungen die Definition seines individuellen Wohls, an das der Betreuer bei der Ausführung der Betreuung nach \1901 Abs. 2 und 3 gebunden ist, ${ }^{492}$ nicht aber die Bestimmung des Betreuungsumfangs.

\section{B. Verfahrenspfleger gem. \276 FamFG}

„Das Gericht hat dem Betroffenen einen Verfahrenspfleger zu bestellen, wenn dies zur Wahrnebmung der Interessen des Betroffenen erforderlich ist.", vgl. \276 Abs. 1 S. 1 FamFG ( $\int 67$ Abs. 1 S. 1 FGG). Damit stellt das Gesetz einen Grundsatz auf. Immer dann, wenn der Betroffene seine Interessen im Verfahren nicht selber ausreichend wahrnehmen kann, ist ein Verfahrenpfleger zu bestellen. ${ }^{493}$ Die Bestellung soll zum Schutz des Betreuten erfolgen. ${ }^{494}$ Weil der Betroffene dazu aufgrund seiner körperlichen, geistigen oder seelischen Behinderung nicht in der Lage ist, soll der Verfahrenpfleger ergänzend und erforderlichenfalls an Stelle des Betroffenen dessen Rechte im Verfahren wahrnehmen. ${ }^{495}$ Dabei geht es insbesondere um das in Art. 103 Abs. 1GG verankerte Recht auf rechtliches Gehör. ${ }^{496}$ Der Verfahrenspfleger soll den Betroffenen grundsätzlich unterstützen, nicht ersetzen. ${ }^{497}$

489 Epple BWNotZ 92, 27, 28.

${ }^{490}$ Epple BWNotZ 92, 27, 28.

491 Vgl. nur MünchKommBGB/Schwab \1896 Rn. 65; Soergel/Zimmermann \1896 Rn. 43 ff.

492 Vgl. hier die Ausführungen unter $\$ 2$ C. II.

${ }^{493}$ Es kommt also grundlegend auf die Frage der Erforderlichkeit an (Keidel/Kuntze/-

Winkler/Kayser \67 FGG Rn. 1). Ein Verfahrenspfleger ist nötig, damit die „erforderliche Wahrung der Belange der Betroffenen" gesichert ist, vgl. BT-Drucks. 11/4529 S. 171.

${ }^{494}$ Bumiller/Winkler \67 FGG Rn. 1; Jansen/Sonnenfeld 67 FGG Rn. 2; BT-Drucks. 11/4528 S. $89,171$.

495 Keidel/Kuntze/Winkler/Kayser \$ 67 FGG Rn. 5; Grell RPfleger 1993, 321, 322; Dodegge/Roth A Rn. 124; Bork FamRZ 2002, 67, 70; KG FamRZ 2000, 1300; FamRefK/Maurer 50 FGG Rn. 6 f.; Pobl BtPrax 1992, 20, 21, 24: Sein Wirkungskreis wird nicht ausdrücklich definiert, sondern bezieht sich laut Gesetz auf die Wahrnehmung der Rechte im Verfahren.

496 Bork FamRZ 2002, 67, 71; Bassenge/Roth \67 FGG Rn. 2; Damrau/Zimmermann \67 FGG Rn. 10; BayObLG FamRZ 1990, 542, 543; LG München I FamRZ 1995, 1440, 1441. Teilweise wird argumentiert, dass die Funktion des Verfahrenspflegers nicht in der Wahrnehmung des rechtlichen Gehörs liege. Vielmehr solle er vermeiden, dass der Betroffene zu einem Objekt des Verfahrens werde (Bienwald/Sonnenfeld/Hofmann \67 FGG Rn. 5 ff.). Die Gewähr des rechtlichen Gehörs zielt jedoch auf die Vermeidung der Degradierung des Betroffenen zu einem Objekt des Verfahrens. Insofern stellt die v.a. von Bienwald dargelegte Argumentation keine Gegenmeinung, sondern eher einen anderen Begründungsansatz dar. Dieser ist jedoch als Grund- 
Für die Frage der Erforderlichkeit stellt $\int 276$ Abs. 1 S. 2 FamFG ( 67 Abs. 1 S. 2 FGG) Regelbeispiele auf. Bis zum 1. BtändG war die Bestellung in diesen Fällen zwingend. Auch in der jetzigen Fassung werden die Beispiele mehrheitlich als zwingend angesehen. ${ }^{498}$

Im Einzelnen ist fraglich, ob der Betroffene durch die Betreuungsverfügung eine konkrete Person für das Amt des Verfahrenspflegers verbindlich bestimmen und ausschließen kann. Weiter ist zu untersuchen, ob die Bestellung eines Verfahrenspflegers durch den Betroffenen vollständig unterbunden werden kann.

\section{Vorschlag in der Betreuungsverfügung}

Damit der Betroffene auf die Auswahl der Person des Verfahrenspflegers Einfluss nehmen kann, dürfen die gesetzlichen Regelungen nicht verbieten, dass der Betroffene bestimmte Personen zum Verfahrenspfleger vorschlägt, bzw. von dieser Tätigkeit ausschließt.

\section{Auswabl einer Person}

Zur Auswahl des Verfahrenspflegers bestimmt \ 276 Abs. 3 FamFG, dass derjenige, der Verfahrenspflegschaften im Rahmen seiner Berufsausübung führt nur dann zum Verfahrenspfleger bestellt werden soll, wenn keine andere geeignete Person zur Verfügung steht, die zur ehrenamtlichen Führung der Verfahrenspflegschaft bereit ist. 499

Eine $\int 1897$ Abs. 4 und $\int 1901$ c vergleichbare Regelung sowie einen Verweis auf diese kennt das FamFG nicht. Dies erlaubt nur bedingt Rückschlüsse auf den möglichen Einfluss des Betroffenen auf die Auswahl des Verfahrenspflegers, weil das Institut der Verfahrenspflegschaft nur minimal gesetzlich geregelt ist. ${ }^{500}$ Gegen einen direkten Einfluss des Betroffenen auf die Auswahl des Verfahrenspflegers spricht, dass der Verfahrenspfleger von Rechts wegen „nur“ die objektiven Inte-

annahme zu sehen. Aus ihm erwächst die Notwendigkeit des rechtlichen Gehörs (Bork FamRZ 2002, 67, 71).

497 BT-Drucks. 11/4528 S. 171; Bumiller/Winkler \67 FGG Rn. 1; Gernhuber/Coester-Waltjen, \ 76

Rn. 31, 38-40; Jürgens/Kröger/Marschner/Winterstein Rn. 349; Grell RPfleger 1993, 321, 322; generell: BVerfG FamRZ 1999, 85, 87; BayObLG FamRZ 2000, 1443, 1443 m.w.N.

498 MünchKommZPO/Schmidt-Recla \276 FamFG Rn. 8; Zum FGG: Pobl BtPrax 1992, 20, 21 f.

499 \67 Abs. 1 S. 6 FGG erklärte $₫ 1897$ Abs. 6 für entsprechend anwendbar. Im Sinne von

$\int 67$ FGG konnte Verfahrenspfleger sowohl ein Verein oder die zuständige Behörde sein (Jansen/Sonnenfeld \$ 67 FGG Rn. 43; Bienwald/Sonnenfeld/Hoffmann \67 FGG Rn. 53; Bienwald Rpfleger 1999, 429). Das LG Braunschweig war anderer Ansicht (FamRZ 2005, 304 mit ablehnender Anm. Bienwald): Auch der Vereins- oder der Behördenmitarbeiter als Vereins-/ Behördenverfahrenspfleger müsse nach den Änderungen des 2. BtÄndG gem. \67a FGG ernennbar sein. Der fehlende Verweis zu $₫ 1897$ Abs. 2 sei als Redaktionsversehen zu sehen gewesen (Jansen/Sonnenfeld \$ 67 FGG Rn. 44).

500 Bienwald/Sonnenfeld/Hoffmann \67 FGG Rn. 1 f. 
ressen des Betroffenen wahrnehmen soll. ${ }^{501}$ Er soll die Verfahrensrechte des Betroffenen an dessen Stelle geltend machen. Wunsch und Wille des Betroffenen soll der Verfahrenspfleger dem Gericht zur Erkenntnis mitteilen, ${ }^{502}$ sein Handeln aber nicht danach ausrichten. Durch die reine Wahrnehmung der objektiven Interessen scheint die Tätigkeit des Verfahrenspflegers von persönlichen Einflüssen und somit auch von persönlichen Bindungen losgelöst. Demnach würde er zur reinen Missbrauchskontrolle in Bezug auf die Betroffenenrechte dienen.503 Als solche müsste ein Einfluss des Betroffenen auf die Auswahl des Verfahrenspflegers nicht notwendig sein. Indem der Verfahrenspfleger die Verfahrensrechte des Betroffenen $^{504}$ ausübt, übt er auch dessen Recht auf Selbstbestimmung aus. Der subjektive Einfluss auf das Auswahlrecht dieser Person sollte daher möglich sein. Dies gilt umso mehr, weil der Verfahrenspfleger gesetzlicher Vertreter des Betroffenen wird $^{505}$ und gerichtlich weisungsunabhängig 506 ist.

Die Weisungsunabhängigkeit bedeutet, dass der Verfahrenspfleger bei der Wahrnehmung der Verfahrensrechte weder an die Wünsche und den Willen des Betroffenen noch an Weisungen des Gerichts gebunden ist. ${ }^{507}$ Auch wenn er (nur) die Verfahrens- und keine sonstigen Rechte des Betroffenen wahren soll, hat bzw. kann er Einblick und v.a Einfluss in höchstpersönliche Entscheidungen des Betroffenen haben. Mit Blick auf die Missbrauchskontrolle kann eine Person des Vertrauens die objektiven Interessen des Betroffenen - nicht nur aus der Perspektive des Betroffenen - interessengerechter, weil auf Basis des Vertrauens, wahrnehmen. ${ }^{508}$ Das subjektive Empfinden des Betroffenen sollte an dieser Stelle nicht unbeachtet bleiben. Dies ist nur konsequent, weil der Wunsch bezüglich der Person des Verfahrenspflegers auch dann für die gerichtliche Ernennungsentscheidung maßgeblich ist, wenn der Betroffene sich noch aktiv äußern kann. ${ }^{509}$ Solange eine Kommunikation mit dem Betroffenen möglich ist, muss das Gericht ihn aufgrund seines Selbstbestimmungsrechts nach der persönlichen Wahl eines Ver-

\footnotetext{
501 BT-Drucks. 11/4528 S. 171; Rogalla BtPrax 1993, 146, 147 f.; Jürgens/Kröger/Marschner/ Winterstein Rn. 348.

502 BT-Drucks. 11/4528 S. 171.

503 Die Notwendigkeit der Missbrauchskontrolle bestätigend Schumacher ZRP 1989, 7, 8 f.

${ }^{504}$ Er ist gesetzlicher Vertreter des Betroffenen: Bork. FamRZ 2002, 67, 70; OLG Frankfurt FamRZ 2000, 1446.

505 Bienwald/Sonnenfeld/Hoffmann \67 FGG Rn. 16.

506 Jürgens/Kröger/Marschner/Winterstein Rn. 348; BT-Drucks. 11/4528 S. 171; Zimmermann FamRZ 1991, 270, 271; Bienwald/Sonnenfeld/Hoffmann \67 FGG Rn. 16.

507 BT-Drucks. 11/4528 S. 171.

508 Daher sind insbesondere solche Personen zu bestellen, die die Lebensverhältnisse des Betroffenen kennen (Zimmermann BtPrax 1992, 19, 23) sowie Vertrauenspersonen aus dem Familienund Bekanntenkreis (Jansen/Sonnenfeld \67 FGG Rn. 38); dazu kritisch: Knittel \67 FGG Rn. 20; Rechtskenntnisse des Verfahrenspflegers sind dabei zwar erwünscht, der persönliche Kontakt wird jedoch als wichtiger eingestuft, vgl. Jansen/Sonnenfeld \67 FGG Rn. 38; Bienwald Verfahrenspflegschaftsrecht Rn. 171.

509 Zimmermann FamRZ 1991, 270, 274.
} 
treters befragen. Trotz fehlender ausdrücklicher gesetzlicher Bestimmung ist die Verpflichtung des Betreuungsgerichts, sich bei der Auswahl des Verfahrenpflegers an dem Vorschlag des Betroffenen zu orientieren und ihn dazu anzuhören, allgemein anerkannt. ${ }^{510}$ Während die herrschende Meinung eine „Orientierung“ verlangt, wird diese Verpflichtung vereinzelt aus der entsprechenden Anwendung der für die Bestellung eines Betreuers geltenden \1897, \278 FamFG (〔68 FGG) abgeleitet. 511 Wenn der Betroffene also aktiv einen Wunschverfahrenspfleger benennen kann, muss dies auch für antizipierte Äußerungen in Form der Betreuungsverfügung gelten. ${ }^{512}$

Im formellen Verfahren ist keine Wunschbeachtungspflicht entsprechend der

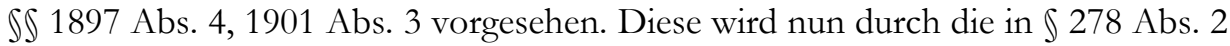
S. 3 FamFG neu eingeführte Pflicht des Gerichts, mit dem Betroffenen die Frage zu erörtern, welche Person als Betreuer in Betracht kommt auch im Verfahren berücksichtigt. Nach den Grundsätzen des Betreuungsrechts war der Einfluss des Betroffenen auf die Auswahl des Verfahrenspflegers bereits zuvor möglich. 513

Zusammenfassend ist festzustellen, dass die Auswahl des Verfahrenspflegers nach \67 FGG entsprechend \1897 Abs. 4 durch den Betroffenen rechtswirksam vorgenommen werden kann. ${ }^{514}$ Der Wille des Betroffenen bei der Auswahl des Verfahrenspflegers hat demnach entsprechend \ 1897 Abs. 4 S. 2 Geltung. ${ }^{515}$

\section{Ausschluss einer Person}

Die rechtliche Bewertung eines Wunsches des Volljährigen, der eine konkrete Person vom Amt des Verfahrensbevollmächtigten ausschließen soll, ist gesetzlich nicht geregelt. Mit Blick auf die Grundsätze des Betreuungsrechts kann in diesem Fall nichts anderes gelten als im Fall des Ausschlusses einer bestimmten Person vom Amt des Betreuers nach \ 1897 Abs. 4 S. 2.516 Soweit die Bestellung einer bestimmten Person zum Verfahrenspfleger des Betroffenen, dessen bewussten,

510 Lipp S. 114; Bumiller/Winkler \67 FGG Rn. 5; Jürgens/Mertens \67 FGG Rn. 10.

511 Lipp S. 114.

512 Langenfeld S. 165; Lipp S. 114 und FS Bienwald S. 177, 186; Jansen/Sonnenfeld \ 67 FGG Rn. 42; Keidel/Kuntze/Winkler/Kayser \67 FGG Rn. 1; Bienwald/Sonnenfeld/Hoffmann \1897 Rn. 16; Bienwald FamRZ 2000, 1281; BVerfGE FamRZ 2000, 1280, 1281.

513 Zimmermann behandelt daher z.B. die Frage, ob man sich gegen einen bestellten Verfahrenpfleger wenden kann, weil dieser einem persönlich nicht gefällt, mit Blick auf die besondere Ausrichtung auf die Betroffenenwünsche im materiellen Recht gem. \ 1901, so dass diese auch bei der Bestimmung des Verfahrenspflegers zu berücksichtigen sind (Zimmermann FamRZ 1994, 286). Wenn der Betroffene sich in der Beschwerde gegen die Person des Verfahrenspflegers wenden kann, dann erst recht von vornherein. Siehe hierzu auch Pobl BtPrax 1992, 19, 20 f.

${ }^{514}$ Im Ergebnis ebenso: Dodegge/Roth C. Rn. 171; Epple BtPrax 1993, 156, 159 und BWNotZ 1992, 27, 30.

515 Bumiller/Winkler $\int 67$ FGG Rn. 5; im Übrigen habe der Verfahrenspfleger jedoch nicht den Vorrang des Betroffenenwillens zu beachten, sondern müsse das objektive Interesse des Betroffenen vertreten (Bumiller/Winkler \67 FGG Rn. 1).

516 Vgl. dazu $\$ 3$ A. II. 
ernsthaften und frei von Irrtum und Einfluss Dritter gebildeten Wunsch widerspricht, ist dieser für das Gericht verbindlich. Die Bestellung dieser Person zum Verfahrenspfleger darf daher nicht erfolgen.

II. Vermeidung der Verfahrenspflegschaft durch privatautonome Bevollmächtigung eines Dritten oder Verzicht auf den Verfahrenspfleger

Fraglich ist, ob die Bestellung eines Verfahrenspflegers vermieden werden kann, indem der Betroffene eine dritte Person mit der Wahrnehmung seiner Verfahrensrechte beauftragt oder auf die Zuordnung eines Verfahrenspflegers mittels seiner Betreuungsverfügung generell verzichtet.

\section{Bevollmächtigte Verfahrensvertreter}

Der gesetzlich vorgeschriebene Vorrang privatautonomer Vertretung (vgl. \1896 Abs. 2 S. 2) hat in $\$ 276$ Abs. 4 FamFG (\$ 67 Abs. 1 S. 7 FGG) Ausdruck gefunden. Danach ,soll die Bestellung eines Verfahrenspflegers unterbleiben oder aufgeboben werden, wenn die Interessen des Betroffenen von einem Rechtsanwalt oder von einem anderen geeigneten Verfahrensbevollmächtigten vertreten wird. "In diesem Fall hat der Betroffene seinen Verfahrensvertreter selber ausgesucht und privatautonom zum Vertreter bestellt.

\276 Abs. 1 S. 1 FamFG/ \67 FGG sieht nur bei Erforderlichkeit die Bestellung eines Verfahrenspflegers vor. Die Erforderlichkeit ist unter Berücksichtigung aller Umstände im jeweiligen Einzelfall zu ermitteln. ${ }^{517}$ Von der Erforderlichkeit ist wie in $\ 1896$ Abs. 2 auszugehen, wenn zur Wahrnehmung der Betroffenenverfahrensrechte eine andere Person als der Verfahrenspfleger fehlt.

Nach dem Wortlaut besteht ein zwingender Vorrang anderer Hilfen vor der Bestellung des Verfahrenspflegers. ${ }^{518}$ Dies entspricht der Gesetzessystematik. Die wirksame Bevollmächtigung einer Wunschperson zur Wahrnehmung der Betroffenenverfahrensrechte ist somit möglich. Die Bestellung eines Verfahrenspflegers ist dann nicht mehr erforderlich. Die Bevollmächtigung kann im Rahmen der Betreuungsverfügung erfolgen.

Bei Unwirksamkeit dieser Bevollmächtigung muss die Auswahl des Betroffenen für den Verfahrensbevollmächtigten durch Umdeutung der Bevollmächtigung gem. $\int 140$ übernommen werden. Andernfalls würde man die Wünsche des Betroffenen missachten.

\footnotetext{
517 Bumiller/Winkler \67 FGG Rn. 2; Jansen/Sonnenfeld \67 FGG Rn. 11; Bienwald/Sonnenfeld/Hoffmann \67 FGG Rn. 28.

518 Pobl BtPrax 1992, 20, 21.
} 


\section{Verzicht auf den Verfabrenspfleger}

Fraglich bleibt, ob der Betroffene in der Betreuungsverfügung die Ernennung eines Verfahrenspflegers ausdrücklich ausschließen kann.

a) Bestellung eines Verfahrenspflegers trotz Verfahrensbevollmächtigten

Der in $\ 276$ Abs. 4 FamFG ( $₫ 67$ Abs. 1 S. 7 FGG) gesetzlich formulierte Vorrang des privatautonom beauftragten Vertreters wird nicht nach allen Meinungen als zwingend angesehen. ${ }^{519}$ Vielmehr soll die Bestellung eines Verfahrenspflegers in bestimmten Fällen trotz vorhandener Bevollmächtigung nicht ausgeschlossen sein. ${ }^{520}$ Bei ständigem Wechsel des Rechtsanwalts ${ }^{521}$ oder des Verfahrensbevollmächtigten ${ }^{522}$ wurde eine Bestellung für notwendig erachtet, bzw. dann, wenn der Betroffene dem Verfahrensbevollmächtigten unsinnige Anweisungen erteilt. ${ }^{523}$ Anders als der Verfahrenspfleger ist dieser dem Betroffenen weisungsgebunden. ${ }^{524}$ In diesen Fällen kann der Betroffene sich nach herrschender Meinung zur früheren Regelung $\ 67$ Abs. 1 S. 7 FGG nicht durch die Benennung eines Verfahrensbevollmächtigten vor der Ernennung eines Verfahrenspflegers schützen. Dies wird auch für den gleich lautenden $\ 267$ Abs. 4 FamFG gelten.

b) Bestellung eines Verfahrenspflegers gegen den Betroffenenwillen

Nach $₫ 276$ Abs. 1 S. 2 FamFG ( 67 Abs. 1 S. 2, 3 FGG) ist die Bestellung eines Verfahrenspfleger in der Regel erforderlich, wenn von der Anhörung des Betroffenen abgesehen werden soll, weil hiervon erhebliche Nachteile für die Gesundheit des Betroffenen zu besorgen sind ${ }^{525}$ oder wenn ein Betreuer zur Besorgung aller Angelegenheiten bestellt werden oder die Erweiterung des Aufgabenkreises hierauf erfolgen soll. Als Regelbeispiele zählt Satz 2 nur Beispielsfälle auf. Das Gericht hat in dieser Entscheidung einen Ermessensspielraum.526 Weiter ist $\ 276$ Abs. 2 FamFG zu berücksichtigen. Danach kann von der regelhaften Verfahrenspflegerbestellung nach Abs. 1 S. 2 abgesehen werden, wenn ein Interesse des Betroffenen an der Bestellung des Verfahrenspflegers offensichtlich nicht besteht.

519 Bassenge/Roth \67 FGG Rn. 6; wohl anders MünchKommZPO/Schmidt-Recla 276 FamFG Rn. 5.

${ }^{520}$ BT-Drucks. 11/4528 S. 171, 231 und 11/6949 S. 78; Bumiller/Winkler $₫ 67$ FGG Rn. 4.

${ }^{521}$ BT-Drucks. 11/4528 S. 171; Keidel/Kuntze/Winkler/Kayser \67 FGG Rn. 16.

522 Bassenge/Roth \ 67 FGG Rn. 6.

523 Bassenge/Roth \67 FGG Rn. 6.

${ }^{524}$ Der Verfahrenspfleger ist kein Amtsträger, weshalb es auch anders als beim Betreuer keinen Bestellungsakt gibt, vielmehr reicht die Bekanntmachung nach $₫ 40$ Abs. 1 FamFG/ $\int 16$ Abs. 1 FGG (Rogalla BtPrax 1993, 146, 146).

525 \276 Abs. 1 Nr. 1 i.V.m. \278 Abs. 4 i.V.m. \34 Abs. 2 FamFG.

526 Bumiller bewertet dies anders, sie beschreibt die Aufzählung in $₫ 267$ Abs. 1 S. 2 FamFG als Fälle, in denen durch das Gericht zwingend ein Verfahrenspfleger bestellt werden muss (Bumiller/Harders $\ 267$ FamFG Rn. 5). 
Die bis zur Einführung des FamFG als zwingend formulierten jetzigen Regelbeispiele werden von der herrschenden Meinung zum FGG als zwingend angesehen. ${ }^{527}$ Die Regelung \ 67 Abs. 1 S. 2 FGG entspricht der Formulierung von \ 276 Abs. 1 S. 2 FamFG. Danach dürfte eine anders lautende Formulierung einer Betreuungsverfügung keine verbindliche Wirkung haben. Abs. 2 erlaubt jedoch ein Abweichen von der in Abs. 1 vorgesehen Bestellung eines Verfahrenspflegers, wenn der Betroffene daran offensichtlich kein Interesse hat. Diese Ausnahme muss unabhängig davon gelten, ob man die Beispiele als zwingend ansieht. Folgt man diesem Gedanken, enthält die Norm auch nach dem FamFG einen Widerspruch. Die Verfassungsmäßigkeit dieser Regelung wird daher auch zu Recht in Frage gestellt. ${ }^{528}$

Zudem steht die Ausnahme des Abs. 2 im Widerspruch zu Abs. 1 S. 1. Nach dem Wortlaut von Abs. 1 S. 1 hat das Gericht bei Erforderlichkeit einen Verfahrenspfleger zu bestellen und somit gerade keinen Ermessensspielraum, nach Abs. 2 kann es von der Bestellung absehen, wenn ein Interesse des Betroffenen an der Bestellung offensichtlich nicht besteht.

Allerdings muss jede natürliche Person auf ihre Rechte und folglich auch auf den Beistand durch den Verfahrenspfleger verzichten können. Sinn und Zweck der Einführung des Rechtsinstitutes im Rahmen des Betreuungsrechts ${ }^{529}$ ist, einem Schutzbedürftigen eine Kontrollinstanz gegenüber dem Staat an die Hand zu geben. ${ }^{530}$ Gerade derjenige, der nach Satz 1 seine Interessen nicht ausreichend wahrnehmen kann, bedarf Beistand zur Wahrung seiner Rechte. Dies gebietet schon der rechtsstaatliche Grundsatz auf ein faires Verfahren. 531 Objektiv gesehen kann somit Satz 3 keine Anwendung finden. Die Bestellung eines Verfahrenspflegers sieht das Gesetz in den von Satz 2 genannten Fällen zwingend vor. Die Vermeidung der Verfahrenpflegerbestellung durch die Betreuungsverfügung ist somit im Ergebnis nicht möglich. Wie bei der Betreuerbestellung hat diese bei Erforderlichkeit zu erfolgen.

\footnotetext{
527 BT-Drucks. 11/4528 S. 171; Epple BtPrax 1993, 156, 159; Keidel/Kuntze/Winkler/Kayser \67 FGG Rn. 5, 9; Pobl BtPrax 1992, 19, 21 f.; Zimmermann FamRZ 1991, 270, 274; Bumiller/Winkler \67 FGG Rn. 3; Gernhuber/Coester-Waltjen \76 Rn. 38-40; Bork FamRZ 2002, 65, 69; Jürgens/Mertens \67 FGG Rn. 4; Bienwald/Sonnenfeld/Hoffmann \67 FGG Rn. 36, 44; Ständeke-Otto BtPrax 1993, 16.

${ }^{528}$ Vgl. nur Jansen/Sonnenfeld \67 FGG Rn. 3, 4, 27; Keidel/Kuntze/Winkler/Kayser \67 FGG Rn. 8 f.; Bauer/Rink BtPrax 1996, 158; Bienwald Verfahrenpflegschaftsrecht Rn. 97; Gernhuber/Coester-Waltjen \$ 76 Rn. 38-40; verfassungsrechtlich bedenklich, weil S. 3 nun eine weit gefasste Ausnahme normiert.

${ }^{529}$ Eine $₫ 67$ FGG vergleichbare Vorschrift gab es vor der Reform nicht.

${ }^{530}$ BT-Drucks. 11/4528 S. 89, 171; Jansen/Sonnenfeld \$ 67 FGG Rn. 2.

531 Jansen/Sonnenfeld \$ 67 FGG Rn. 27.
} 
III. Vergütung des Verfahrenspflegers

Fraglich ist, ob der Betroffene mittels der Betreuungsverfügung das $\mathrm{Ob}$ und Wie der Vergütung des Verfahrenspflegers bestimmen kann. Die Vergütung des Verfahrenspflegers war lange umstritten; erst durch das 2. Bt ÄndG ${ }^{532}$ wurde sie in \67a Abs. 3 S. 2 FGG positiv geregelt. \67a FGG ist mit der aktuellen Regelung des $\int 277$ FamFG identisch.

Bei einer Bevollmächtigung kann die Vergütung des Verfahrensbevollmächtigten privatrechtlich vereinbart werden. ${ }^{533} \mathrm{Zu}$ untersuchen ist, ob der Betroffene seinen Wunschverfahrenspfleger unabhängig von den gesetzlichen Vorgaben durch privatautonome Vereinbarung vergüten kann. ${ }^{534}$

Eine privatautonome Bestimmung der Vergütung wird durch die geltenden gesetzlichen Bestimmungen nicht ausdrücklich untersagt. \277 Abs. 2 FamFG i.V.m. $\int 1836$ Abs. 1 S. 1 legen fest, dass die Verfahrenspflegschaft grundsätzlich ehrenamtlich und somit unentgeltlich zu führen ist. Nach \1836 Abs. 2 kann der Betroffene eine angemessene Vergütung nur bewilligen, wenn der Umfang oder die Schwierigkeit der Geschäfte dies rechtfertigen und er nicht mittellos ist.

Nur im Ausnahmefall kann die Verfahrenspflegschaft gem. \ 277 Abs. 2 FamFG i.V.m. \1836 Abs. 1 S. 2 von einem Berufspfleger und dann auch entgeltlich übernommen werden. Dieser erhält nach $\int 277$ Abs. 2 S. 2 FamFG i.V.m. $\iint 1-3$ Abs. 1 und 3 VBVG neben der für ehrenamtlich Tätige gem. \ 277 Abs. 1 FamFG i.V.m. \1835 Abs. 1, 2 gezahlten Aufwandsentschädigung eine Vergütung. ${ }^{535}$ Gem. \ 277 Abs. 5 S. 1, \ 168 Abs. 1 FamFG i.V.m. \ 1835a erfolgt diese aus dem Betreutenvermögen (Mündelvermögen), bei Mittellosigkeit aus der Staatskasse. Der Staat hat dann gem. \1836e einen Regressanspruch gegen den Betroffenen. ${ }^{536}$ Somit zahlt der Betroffene Aufwandsentschädigung und Vergütung des Verfahrenspflegers, soweit vorhanden, aus seinem Vermögen. Das „Ob“ einer Vergütung ist für den Betroffenen daher nicht zwingend festgelegt. Ein Einfluss des Betroffenen kann demzufolge über das gesetzlich zwingende Maß hinaus

532 @ 67a FGG wurde eingeführt mit Wirkung zum 01.07.2005, vgl. dazu Fn. 70.

533 Bienwald Verfahrenpflegschaftsrecht Rn. 659. Bei Rechtsanwälten stellt sich dann die Frage der Regulierung durch das BRAG ( $₫ 1$ Abs. 2 S. 1 RVO) - so ist eine Abrechnung nach der BRAG untersagt, wenn er Berufsverfahrenspfleger ist (Krauß BWNotZ 2006, 35, 37; Schlöpke Rpfleger 1993, 435; Damrau/Zimmermann \70b FGG Rn. 5; Jürgens/Kröger/Marschner/Winterstein \ 70b FGG Rn. 360; Bienwald/Sonnenfeld/Hoffmann \70b FGG Rn. 15); siehe \5 B. Sollte ein Rechtsanwalt jedoch nur privatautonom beauftragt werden, müsste die Abrechnung regulär nach BRAG erfolgen.

534 Str. - so zumindest auch Epple BWNotZ 1992, 27, 29 f. in Bezug auf den Betreuer. Kritisch dazu Bienwald Verfahrenpflegschaftsrecht Rn. 659, wegen Rückforderungsansprüchen des Vermögensbetreuers.

535 Er erhält jedoch nicht den Betreuervergütungsstundensatz, sondern den (niedrigeren) Vormündervergütungssatz. Tatsächlich aufgewandte Zeit - je nach Ausbildung 19, $50 €-25,00 €-$ 33, $50 €$ zzgl. Umsatzsteuer und Aufwendungsersatz.

536 Bienwald Verfahrenspflegschaftsrecht Rn. 657. 
diskutiert werden. ${ }^{537}$ Die Disponibilität ist aber auf die Fälle der vermögenden Betroffenen einzuschränken. ${ }^{538}$ Ein mittelloser Betroffener kann nicht eine individuelle höhere Vergütung bestimmen, die dann aus der Staatskasse und somit aus den Steuergeldern aller Bürger entrichtet wird. ${ }^{539}$ Dies würde eine Verfügung zulasten Dritter darstellen. Dies ist wirksam nicht möglich.

Die Vergütungsstruktur des Verfahrenspflegers ist mit der des Betreuers vergleichbar. ${ }^{540}$ Soweit eine Vergütung erfolgt, wird diese in beiden Fällen nach den Regelungen des VBVG gewährt. ${ }^{541}$ Daher ist in der Frage der individuellen Vergütungsvereinbarung grundsätzlich auf die dort geführte Diskussion zu verweisen. ${ }^{542}$ Als Unterschied ist zu beachten, dass der Betreuer sein Handeln gem. \ 1901 an dem Wunsch und Willen des Betroffenen auszurichten hat. ${ }^{543}$ Der Verfahrenspfleger ist hingegen nur an die objektiven Interessen des Betroffenen gebunden. Er ist ausdrücklich weder gegenüber dem Betroffenen noch dem Gericht weisungsgebunden. ${ }^{544}$ Dies führt jedoch nicht dazu, dass der Betroffene kein Interesse an der Person und dessen Handeln hat. Als gesetzlicher Vertreter des Betroffenen gelten seine Entscheidungen gem. IS 164 ff. für und gegen den Betroffenen. ${ }^{545}$ Auf sie, wie auf die grundsätzliche Frage der Bestellung des Verfahrens-

537 Somit ist auch die Vergütung eines ehrenamtlich Tätigen durch einen vermögenden Betreuten disponibel. Dies strikt verneinend: HK-BUR/Bauer \$ 67a FGG Rn. 28.

538 Zum Begriff der Mittellosigkeit vgl. Staudinger/Bienwald \ 1836d Rn. 2 ff. Das Recht zur Anfechtung der Bestellung eines Verfahrenspflegers gilt daher auch nur für vermögende Betroffene, vgl. etwa LG Lübeck RPfleger 1994, 110, 111.

539 Andeutend OLG Oldenburg FamRZ 2005, 391, 391.

540 Kirch RPfleger 1992, 379 ff. m.w.N.; kritisch dazu Rogalla BtPrax 1993, 146, 149. Jedoch ist zu beachten, dass der Verfahrenspfleger nach $\iint 1$ - 3 Abs. 1, 3 VBVG ( 67 a Abs. 2 S. 2 FGG) nicht den Betreuervergütungsstundensatz, sondern den (niedrigeren) Vormündervergütungssatz erhält, d.h. konkret die tatsächlich aufgewandte Zeit - je nach Ausbildung 19, 50 - 25, 00 33, $50 €$ zzgl. Umsatzsteuer und Aufwendungsersatz. Da auf $\int 3$ Abs. 3 VBVG nicht verwiesen wird, kann auf die besondere Schwierigkeit der Sache und bei Vermögen des Mündels (Betroffenen) kein höherer Stundensatz bewilligt werden. Das Gericht kann statt der Stundenabrechnung einen festen Geldbetrag („Individualpauschale“) zubilligen, $\int$ 67a Abs. 3 S. 1 FGG. Dabei wird die voraussichtlich notwendige Zeit mit dem Stundensatz multipliziert und der Stundensatz um $3 €$ erhöht (= Aufwandspauschale, dafür bedarf es keiner Einzelauflistung der Tätigkeit).

541 So bezieht sich die gesetzgebende Diskussion z.B. auch ausdrücklich auf dieselben Gründe für den Vorrang der ehrenamtlichen vor der berufsmäßigen Betreuung, vgl. nur BT-Drucks. 15/2494 S. 18; Krauß BWNotZ 2006, 35, 35, 37.

542 Siehe $\int 5 \mathrm{~B}$.

543 Soweit es mit der Funktion des Verfahrenspflegers vereinbar ist, gelten die allgemein betreuungsrechtlichen Regelungen (Lipp S. 114).

544 Jürgens/Kröger/Marschner/Winterstein Rn. 348; Lipp S. 111 f.; Damrau/Zimmermann \ 67 FGG Rn. 30; als objektiver Beteiligter muss er auch den Richter kontrollieren. V.a. mit Blick auf die zukünftige Bestellung kann dies seiner originären Aufgabe ggf. nicht zuträglich sein, da seine Bestellung alleine im Ermessen des Betreuungsgericht, und damit dem zuständigen Richter steht, vgl. dazu nur Knieper JurBüro 1998, 289, 290 f.

545 Der Verfahrenspfleger greift in die Rechtsphäre des Betroffenen ein, weshalb er auch zwingend Verfahrensbeteiligter sein muss, vgl. nur Lipp S. 111 f.; Kirsch RPfleger 1992, 379, 381. 
pflegers hat der Betroffene keinen Einfluss. ${ }^{546}$ Wie bereits oben besprochen, vertraut der Betroffene einem Wunschverfahrenspfleger auch in der Wahrnehmung seiner objektiven Interessen leichter, als einem Unbekannten. Die Frage individueller Vergütung ist trotz gesetzlich geregelter Pauschalen mithilfe des Selbstbestimmungsrechts zu beantworten. Dieses erlaubt jedem Einzelnen, sein Vermögen nach subjektiven Kriterien zu verwalten und folglich auch zu verteilen. ${ }^{547}$ Selbst der aufgrund geistiger, körperlicher oder seelischer Behinderung in der Vermögensverwaltung Eingeschränkte und daher Betreute behält diese Grundfreiheit. 548 Dies kann einem Betroffenen, für den Zeitraum der Prüfung der Notwendigkeit einer Betreuerbestellung erst recht nicht abgesprochen werden. Dies gilt v.a. auch mit Blick auf die uneingeschränkte Verfahrensfähigkeit des Betroffenen gem. \275 FamFG (\66 FGG).

Der privatautonome Einfluss des vermögenden Betroffenen muss über den Mindestsatz der gesetzlich geregelten Vergütung hinaus nach den gesetzlichen Regelungen möglich sein. Der Betroffene kann daher auch einem ehrenamtlichen Verfahrenspfleger eine Vergütung zukommen lassen. ${ }^{549}$

Nach der gesetzlichen Regelung erhalten Betreuungsbehörden für die Verfahrenspflegerbestellung eines Mitarbeiters keine Vergütung und keinen Aufwendungsersatz, vgl. \ 277 Abs. 4 S. 3 FamFG. ${ }^{550}$ Werden Mitarbeiter eines Vereins bestellt, stehen dem Verein gem. \277 Abs. 4 S. 1 FamFG Vergütung und Aufwendungsersatz zu.

Anders das Bundesverfassungsgericht, welches entschied, dass eine Grundrechtsverletzung der Berufsausübungsfreiheit vorliege, wenn einem Betreuungsverein jegliche angemessene Entschädigung für die Wahrnehmung einer Verfahrenspflegschaft durch einen Mitarbeiter vorenthalten werde, der bei ihm beschäftigt wird, um Betreuungen und Pflegschaften auf Grund gerichtlicher Bestellung zu übernehmen. ${ }^{551}$ Der Bundesgerichtshof hat sich dieser Überlegung für die Tätigkeit eine Vereinsmitglieds als Pfleger in analoger Anwendung \67a Abs. 4 FGG angeschlossen und die fehlende gesetzliche Normierung als planwidrige Regelungslücke bewertet. 552

Diese Regelungen sind jedoch als zwingend anzusehen, weil die Zahlung von Aufwendungsersatz und Vergütung der Verfahrenspfleger gem. \ 277 Abs. 5 FamFG stets aus der Staatskasse erfolgt, so dass der Betroffene darauf keinen

\footnotetext{
${ }^{546}$ Vgl. \4 B. I. und Bienwald, Verfahrenspflegschaftsrecht Rn. 71 ff.

$547 \mathrm{Vgl}$. Ausführungen in $₫ 2 \mathrm{C}$.

548 Vgl. nur Lipp S. 161.

549 Allein der fehlende Verweis auf $\int 1836$ Abs. 2 rechtfertigt hier keine Beschränkung des Selbstbestimmungsrechts des Betroffenen, so aber KraußBWNotZ 2006, 35, 37.

550 Ebenso: Müller ZKJ 2007, 449; anders: BVerfG FamRZ 2000, 414 f. und BGH NJW-RR 2007, 937, 938; anders, jedoch eher die Frage der Vergütung eines Vereins beurteilend: OLG Brandenburg FamRZ 2003, 882, 883.

551 BVerfG FamRZ 2000, 414 f.

552 BGH NJW-RR 2007, 937, 938.
} 
Einfluss nehmen kann. Werden hingegen Mitarbeiter eines Betreuungsvereins bestellt, die gem. \277 Abs. 4 S. 1 FamFG eine Vergütung abrechnen können, müsste die Einflussnahme durch den Betroffenen nach oben gesagtem möglich sein.

\section{Anhörung des Betroffenen gem. \$278 FamFG}

Fraglich ist, inwieweit die Ausgestaltung der Anhörung eine Einflussnahme des Betroffenen gewährt. Zu untersuchen ist, ob er den Ort der Anhörung sowie die Auswahl der anwesenden Personen für das Gericht verbindlich festlegen, bzw. die Anhörung verhindern kann.

\section{Unterbinden einer Anhörung}

„Das Gericht hat den Betroffenen vor der Bestellung eines Betreuers [...] persönlich anquhören. Es hat sich einen persönlichen Eindruck von dem Betroffenen zu verschaffen. "vgl. \ 278 Abs. 1 S. 1 und 2 FamFG. Die Regelung entspricht inhaltlich dem früher geltenden \68 FGG. Gem. \278 Abs. 4 i.V.m. \34 Abs. 2 FamFG kann das Gericht von der persönlichen Anhörung in zwei Fällen absehen. ${ }^{553}$ Die erste Ausnahme liegt vor, wenn nach ärztlichem Gutachten von der Anhörung erhebliche Nachteile für die Gesundheit des Betroffenen zu besorgen sind. In diesem Fall ist das Gericht jedoch nicht von der Verpflichtung entbunden, sich einen unmittelbaren Eindruck von dem Betroffenen zu verschaffen. Aus $\int 26$ FamFG ( $₫ 12$ FGG) folgt seine Pflicht, die zur Feststellung der entscheidungserheblichen Tatsachen erforderlichen Ermittlungen durchzuführen. ${ }^{54}$ Die durch die Anhörung zu befürchtenden Nachteile müssen erheblich sein. ${ }^{555}$ Können gesundheitliche Gefahren durch Medikamente vermieden werden, müssen diese eingesetzt werden. Es muss sich um ein ärztliches Gutachten handeln, aufgrund dessen von der Anhörung abgesehen wird. ${ }^{556}$ An das Gutachten ist der Richter nicht gebunden, er muss selber entscheiden, ob eine Anhörung möglich ist. ${ }^{557}$

Die zweite Ausnahme von der persönlichen Anhörung ist dann gegeben, wenn der Betroffene offensichtlich nicht in der Lage ist, seinen Willen kundzutun. 558

553 Vereinzelt wird überlegt, ob der unmittelbare Eindruck ein Teil der Anhörung ist, vgl. Bienwald/Sonnenfeld/Hoffmann \68 FGG Rn. 14.

554 Zöller/Feskorn \26 FamFG Rn. 6; Damrau/Zimmermann \68 FGG Rn. 37.

555 Die ernstliche Gesundheitsgefahr muss bestehen, sie ist bei schwerwiegenden, irreversiblen oder lebensgefährlichen Schäden gegeben (siehe mit Beispielen und jeweiligen Nachweisen bei Damrau/Zimmermann \ 68 FGG Rn. 38).

556 Genauen Anforderungen sind unklar, näher: Damrau/Zimmermann \68 FGG Rn. 39 ff.

557 Damrau/Zimmermann $\int 68$ FGG Rn. 40.

558 Nach der früheren Regelung in $\int 68$ Abs. 2 Nr. 2 FGG musste diese Verständigungsunfähigkeit „nach unmittelbaren Eindruck des Gerichts"von diesem selbst festgestellt werden. Dieser Passus, der die Pflicht des Gerichts noch erhöhte, ist in der geltenden Fassung des \34 Abs. 2 FamFG nicht mehr enthalten. 
Eine Anhörung scheidet also aus, wenn der Betroffene nicht mehr in der Lage ist, Fragen zu beantworten oder zu stellen und keine zusammenhängende Sätze mehr bilden kann. ${ }^{559}$

Das Gesetz enthält nur diese zwei Ausnahmen. Diese ermöglichen das Absehen von der Anhörung, verbieten diese jedoch nicht. Die Ausnahmen sind eng gefasst. ${ }^{560}$ Folglich muss in fast allen Fällen eine Anhörung zwingend vorgenommen werden. Nur so kann das gesetzgeberische Ziel, einen nahen Kontakt zwischen Betroffenen und Gericht zu schaffen, verwirklicht werden. ${ }^{561}$

Die Anhörung nach \278 FamFG (\68 FGG) soll dem Betroffenen als spezielle Ausprägung von Art. 103 Abs. 2 GG rechtliches Gehör einerseits gewähren und stellt andererseits eine besonders intensive Art der bindend vorgeschriebenen Sachaufklärung gem. \26 FamFG (\ 12 FGG) dar. ${ }^{562}$ Die Anhörung in der jetzigen Form wurde erst durch das Betreuungsgericht eingeführt. ${ }^{563}$ Sie soll zu einer starken persönlichen Beziehung zwischen dem Gericht und dem Betroffenen und $\mathrm{zu}$ einer optimalen Aufklärung aller entscheidungserheblichen Umstände führen. ${ }^{564}$ In der Anhörung ist v.a. zwingend die Frage zu erörtern, wer Betreuer werden soll ${ }^{565}$ und ob es eines Verfahrenspflegers bedarf. ${ }^{566}$

Die Anhörung ist folglich in jedem Fall zwingend und grundsätzlich von dem zuständigen Gericht durchzuführen. ${ }^{567}$ Sie ist vom erkennenden Richter durchzuführen, vgl. \ 278 FamFG ( 68 Abs. 1 S. 4 und 5 FGG). ${ }^{568}$ In dieses System ist die unter engen Voraussetzungen gefasste Möglichkeit der Amtshilfe nach \ 278 Abs. 3 FamFG (\68 Abs. 1 S. 4 und 5 FGG) einzuordnen. ${ }^{569}$ Nur in diesem Aus-

559 Damrau/Zimmermann \$ 68 FGG Rn. 43.

560 Peters S. 89; BT-Drucks. 11/4528 S. 90.

561 Peters S. 89; BT-Drucks. 11/4528 S. 172.

562 Zimmermann FamRZ 1991, 270, 272; Holzhauer/Reinicke \68 FGG Rn. 1.

563 Nach früherem Recht war eine solche nicht vorgesehen, de lege lata besteht die Anhörung nach \68 Abs. 1 FGG erst mit Einführung BtG.

564 BT-Drucks. 11/4528 S. 172; Damrau/Zimmermann \68 FGG Rn. 23; BVerfG NJW 1990, 2309, 2310.

565 LG Duisburg FamRZ 2006, 146, 147; Dodegge NJW 2006, 2670, 2671.

566 Zimmermann FamRZ 1991, 270, 272.

567 KG FamRZ 1995, 1379; OLG Stuttgart FamRZ 1993, 1365.

568 Nach der Regelung des FGG konnte die Anhörung mit dem sog. Schlussgespräch, der Erörterung gem. \68 Abs. 5 S. 2 FGG zusammengelegt werden, soweit dies sachdienlich und im Interesse des Betroffenen war (BT-Drucks. 11/4528 S. 173). Auch vom Schlussgespräch konnte nur in den besonderen Ausnahmefällen des $₫ 68$ Abs. 1 Nr. 1 oder 2 FGG abgewichen werden (Peters S. 89). Das Gespräch war als Teil der Anhörung zu verstehen; beides sollte der Stärkung der verfahrensrechtlichen Rolle des betroffenen Personenkreises dienen. Mit Einführung des FamFG ist es weggefallen. Nach den Regelungen des FGG, hätten für die Anhörung und das Schlussgespräch dieselben Bedingungen gelten sollen (Peters S. 89; Jürgens/Kröger/Marschner/Winterstein Rn. 372 f.).

${ }^{569}$ Nur im Ausnahmefall ist sie von einem ersuchenden Richter durchzuführen: BayObLG FamRZ 1993, 1225, 1226; BayObLG FamRZ 1993, 450; Bienwald/Sonnenfeld/Hoffmann Vorbem. v. \\} 6 5 \text { ff. FGG Rn. } 1 9 \text { f.; Damrau/Zimmermann \68 FGG Rn. 30; Zimmermann } FamRZ 1991, 270, 272. 
nahmefall ist von der Anhörung abzusehen. ${ }^{570}$ Unterbleibt die Anhörung, ist zwingend ein Verfahrenspfleger zu bestellen. ${ }^{571}$ Weigert sich der Betroffene, kann er vorgeführt werden, vgl. \278 Abs. 5 FamFG (§ 68 Abs. 3 FGG). Ein Einfluss durch den Betroffenenwillen auf das „Ob“ der Anhörung ist daher nicht möglich. Die gesetzlichen Regeln sind ihrem Wortlaut und dem Sinn des Gesetzgebers nach zwingend und abschließend. Der Betroffene kann sich - zu seinem Schutz einer Anhörung nicht entziehen.

\section{Anhörungsort gem. \278 Abs. 1 FamFG}

\ 278 Abs. 1 S. 2 und 3 FamFG (\ 68 Abs. 1 S. 2 FGG) regelt, dass sich das Gericht von dem Betroffenen einen persönlichen Eindruck zu verschaffen hat. „Diesen persönlichen Eindruck soll sich das Gericht in dessen üblicher Umgebung verschaffen, wenn es der Betroffene verlangt oder wenn es der Sachaufklärung dient und der Betroffene nicht widerspricht. "Demnach sieht das Gesetz ausdrücklich eine Einflussnahme des Betroffenen bei der Wahl des Ortes vor, an dem sich das Gericht von ihm einen persönlichen Eindruck verschaffen soll. Nach der Regelung des früheren \68 Abs. 1 FGG hatte sich das Gericht den Betroffenen persönlich anzuhören und sich einen unmittelbaren Eindruck zu verschaffen. Die Änderung des Gesetzestextes soll nach der Gesetzesbegründung sprachlicher Art sein. ${ }^{572}$ Eine inhaltlich von der früheren Regelung des $\int 68$ FGG abweichende Auslegung ist daher nicht vorzunehmen.

Weil die unmittelbare oder persönliche Eindrucksverschaffung grundsätzlich im Rahmen der Anhörung geschieht, ${ }^{573}$ ist die Einflussnahme des Betroffenen auf den Ort der Anhörung ebenfalls anzunehmen. ${ }^{574}$ Umstritten ist, ob dieses Verlangen des Betroffenen verbindlich ist. ${ }^{575}$ Nach einer Meinung ist dies gegeben, weil es das grundsätzliche Ziel des Betreuungsrechts widerspiegelt, den Betroffenen in das Verfahren besser einzubinden. Dies sollte mit verbindlichen Vorschlägen des Betroffenen am ehesten möglich sein. Auch würde dies der materiellrechtlichen Wunschbefolgungspflicht des $\int 1901$ Abs. 3 S. 1 entsprechen und dem Selbstbestimmungsrecht des Betroffenen gerecht werden. Dies ist besonders zu beachten, da es in diesem Zeitpunkt des Verfahrens gerade um die Feststellung der Betreuungsbedürftigkeit geht.

\footnotetext{
570 Zimmermann FamRZ 1991, 270, 273; BT-Drucks. 11/4528 S. 172.

571 Zimmermann FamRZ 1991, 270, 273.

572 BT-Drucks. 16/6308 S. 267.

573 Jansen/Sonnenfeld \68 FGG Rn. 30; Zumindest gilt dies lt. Damrau/Zimmermann \ 68 FGG Rn. 5 für die Praxis; Bienwald/Sonnenfeld/Hoffmann \68 FGG Rn. 5.

574 Andeutungsweise so auch Bienwald/Sonnenfeld/Hoffmann \68 FGG Rn. 16 und Schwab. Nach seinem Verständnis erfolgt die Anhörung grundsätzlich im Gericht, wenn der Betroffene dies aber verlangt, es der Sachaufklärung dient und der Betroffene nicht widerspricht, in dessen unmittelbarer Umgebung (MünchKommBGB/Schwab \1896 Rn. 160).

575 Einen Ermessensspielraum annehmend: OLG Düsseldorf FamRZ 1996, 1373.
} 
Nach anderer Meinung kommt die Anhörung in der gewünschten Umgebung nur in Betracht, wenn dies dem Gericht zweckmäßig erscheint. ${ }^{576}$ Letztlich ist die im Regierungsentwurf vorgesehene Milieuanhörung nicht zu Gesetz geworden, die Anhörung demnach grundsätzlich im Gericht durchzuführen. ${ }^{577}$ Nach ganz überwiegender Meinung ist die Anhörung vom Verschaffen des unmittelbaren bzw. persönlichen Eindrucks zu unterscheiden. Die Verschaffung des unmittelbaren Eindrucks ist zwingend, ${ }^{578}$ kann allerdings - im Gegensatz zur Anhörung, welche nur mündlich stattfinden kann $^{579}$ - auch ohne Worte erfolgen ${ }^{580}$. Ist der Betroffene nicht mehr mobil, finden Eindrucksverschaffung und Anhörung zwangsläufig in der unmittelbaren Umgebung des Betroffenen statt, der sich zumeist an einem selbstbestimmten Ort befindet. \278 Abs. 1 S. 3 ( $\int 68$ Abs. 1 S. 2 FGG) ist eine Soll-Vorschrift. Demnach ist der Ortsvorschlag des Betroffenen nicht zwingend, ihm ist jedoch grundsätzlich zu folgen. S. 3 eröffnet kein freies Ermessen, sondern erlaubt nur begründete Ausnahmen. ${ }^{581}$

Die unmittelbare Eindrucksverschaffung ist zwingender Verfahrensteil. ${ }^{582}$ Sie soll das rechtliche Gehör über die grundsätzlich durchzuführende Anhörung hinaus umsetzen und gleichzeitig zur Wahrung der Intimsphäre des Betroffenen dienen, die ihre Grundlage in Art. 13 GG findet. Durch die unmittelbare bzw. persönliche Eindrucksverschaffung über das Leben des Betroffenen in seiner natürlichen Umgebung wird auf seine Interessen umfassend Rücksicht genommen. Daher kann sie bei einem Widerspruch des Betroffenen gem. $\int 278$ Abs. 1 S. 3 FamFG ( $\int 68$ Abs. 2 S. 2 FGG) auf keinen Fall ${ }^{583}$ in seiner üblichen Umgebung erfolgen. ${ }^{584}$ Dies gilt auch dann, wenn es nach Meinung des Gerichts der Sachaufklärung dient. ${ }^{585}$

Ein verbindlicher Vorschlag würde dem Gericht keinen Entscheidungsspielraum lassen. ${ }^{586}$ Zum einen ist dieser nach der gesetzlichen Formulierung nicht gewollt, zum anderen aber auch nicht zweckmäßig. Nicht in jedem Fall ist die

576 Damrau/Zimmermann \68 FGG Rn. 9; Holzhauer/Reinicke \68 FGG Rn. 4.

577 Vgl. BtG-DiskE S. 170 ff.; BT-Drucks. 11/4528 S. 172; MünchKommBGB/Schwab \1896

Rn. 160.

578 Jansen/Sonnenfeld \68 FGG Rn. 30; Bienwald/Sonnenfeld/Hoffmann \68 FGG Rn. 5.

579 Dodegge/Roth A. Rn. 139; Jansen/Sonnenfeld \68 FGG Rn. 18.

580 Jansen/Sonnenfeld $\int 68$ FGG Rn. 30.

581 Jansen/Sonnenfeld \$ 68 FGG Rn. 34 und damit einen gewissen Spielraum, vgl. OLG Düsseldorf FamRZ 1996, 1373; Knittel \$ 68 FGG Rn. 6; Keidel/Kuntze/Winkler/Kayser \68 FGG Rn. 7;

MünchKommZPO/Schmidt-Recla \278 FamFG Rn. 10 spricht von pflichtgemäßem Ermessen.

582 HK-BUR/Bauer $\ 68$ FGG Rn. 42.

583 Jansen/Sonnenfeld \$ 68 FGG Rn. 35.

584 Verbindlichkeit kann zudem aus Missbrauchsgründen nicht angenommen werden (Damrau/Zimmermann $₫ 68$ FGG Rn. 9). Eine Missbrauchsgefahr erkennt nicht: HK-BUR/Bauer \68 FGG Rn. 66.

585 Einhellige Meinung, vgl. nur Zimmermann FamRZ 1991, 270, 272; Keidel/Kuntze/Winkler/Kayser \68 FGG Rn. 7; BT-Drucks. 11/4528 S. 172.

586 Dieser wird in diesem Fall aber als notwendig angesehen (Keidel/Kuntze/Winkler/Kayser $\int 68$ FGG Rn. 7). 
Anhörung, bzw. die unmittelbare Eindrucksgewinnung an dem vom Betroffenen gewünschten Ort notwendig. Auch mit Blick auf die entstehenden Kosten für einen außergerichtlichen Anhörungstermin wäre die Annahme der Verbindlichkeit des Betroffenenvorschlags nicht immer verhältnismäßig. Demnach kann der Betroffene zwar Vorschläge für den Ort der Anhörung äußern, diese sind für das Gericht jedoch nicht verbindlich. ${ }^{587}$ Dieses hat sich vielmehr unter dem Aspekt der Zweckmäßig- und Verhältnismäßigkeit zur unmittelbaren bzw. persönlichen Eindrucksermittlung an diesem Wunsch zu orientieren und kann ihm demnach entsprechen bzw. ausschlagen und dennoch eine Anhörung und unmittelbare bzw. persönliche Eindrucksverschaffung im Gericht anordnen. Nur die Orientierung am Wunsch des Betroffenen ist für das Gericht zwingend. ${ }^{588}$

III. Hinzuziehung von Vertrauenspersonen und Ausschluss Dritter von der Anhörung gem. $\int \$ 278$ f. FamFG

\section{Hin₹uriehung von Vertrauenspersonen}

Die neue Regelung des $\ 278$ FamFG zur Anhörung des Betroffenen enthält keine Angaben über die gleichzeitige Anwesenheit Dritter. Die Vorgängerregelung \68 FGG regelte in Abs. 4 S. 2 und 3: „Auf Verlangen des Betroffenen ist einer Person seines Vertrauens die Anwesenheit zu gestatten. Anderen Personen kann das Gericht die Anwesenheit gestatten, jedoch nicht gegen den Willen des Betroffenen." Ausschließlich auf Wunsch des Betroffenen sollten Personen seines Vertrauens in die Anhörung einzubeziehen sein. Die Regelung wurde in das FamFG nicht aufgenommen. Dafür wurde $\ 170$ GVG um folgende Sätze ergänzt: „Das Gericht kann die Öffentlichkeit zulassen, jedoch nicht gegen den Willen eines Beteiligten. In Betreunngs- und Unterbringungssachen ist auf Verlangen des Betroffenen einer Person seines Vertrauens die Anwesenheit zu gestatten."

\68 Abs. 4 FGG wurde zum Schutz des Betroffenen in das Gesetz aufgenommen, sollte die Rechte des Betroffenen im Verfahren stärken und eine Vertraulichkeit des Verfahrens herbeiführen. ${ }^{589}$ Aus denselben Motiven wurde $\ 170$ GVG geändert. ${ }^{500}$ Die Anwesenheit der Vertrauensperson war nach dem Wortlaut von $\ 68$ FGG bei entsprechendem Wunsch des Betroffenen zwingend erforderlich. Diese Auslegung fand bis zur Reform des FGG breite Zustimmung. Dem Gericht stand demnach kein Ermessen zu: Nannte der Betroffene eine Vertrau-

\footnotetext{
587 Ähnlich auch die Begründung der jetzigen Regelung, vgl. BT-Drucks. 11/4528 S. 214.

588 So im Ergebnis auch Holzhauer/Reinicke \68 FGG Rn. 4; HK-BUR/Bauer \68 FGG Rn. 68. Anderer Ansicht sind: Damrau/Zimmermann \68 FGG Rn. 7 ff.; Keidel/Kuntze/Winkler \68 FGG Rn. 7; Zimmermann FamRZ 1991, 270, 273.

589 BT-Drucks. 11/4528 S. 172 f. und S. 89 f. (schon im ursprünglichen Entwurf); Dodegge/Roth A. Rn. 148.

${ }^{590}$ Die Gesetzesbegründung stellt dabei auf die Abwägung der Wahrung der Öffentlichkeit im Verfahren als Rechtsstaatsprinzip gegenüber dem Schutz der Privatsphäre des Einzelnen ab, vgl. BT-Drucks. 16/6308 S. 320.
} 
ensperson, die bei der Anhörung anwesend sein sollte, war das Gericht an diesen Wunsch gebunden. ${ }^{591} \mathrm{Da} \int 170$ S. 3 GVG dem \68 Abs. 4 S. 2 FGG entsprechen soll, ${ }^{592}$ sollte dafür Gleiches gelten.

Nach der Gesetzesbegründung ist die Regelung über die Anwesenheit Dritter als Ausnahme von der Nichtöffentlichkeit systematisch im GVG anzuordnen. ${ }^{593}$ Diese Einschätzung ist zur übrigen Einordnung der Regelungen über die Anwesenheit Dritter bei der Anhörung unsystematisch. Nach der gesetzlichen Begründung ist die Regelung des $\int 68$ Abs. 4 S. 2 FGG entbehrlich, da der Betroffene gem. \12 FamFG/ \13 FGG, jederzeit mit einer ibm vertrauten Person als Beistand erscheinen" kann.594 Gegenüber der Regelung des FGG ist dies eine Einschränkung, ${ }^{595}$ da Beistand i.S.v. \ 12 FamFG nur sein kann, wer im Verfahren, in dem die Beteiligten das Verfahren selbst betreiben können, als Bevollmächtigter zur Vertretung befugt ist. Dies beschränkt sich auf die in $\int 10$ Abs. 2 FamFG genannten Personen. ${ }^{596} \mathrm{Da}$ es sich bei der Anwesenheit von Dritten, nach dem Wunsch des Betroffenen gerade nicht um Bevollmächtigte i.S.v. \ 10 FamFG handelt, ermöglicht $\int 12$ FamFG nicht die Hinzuziehung jedweder Vertrauensperson auf Wunsch des Betroffenen mittels der Betreuungsverfügung.

Nach \68a S. 4 FGG sollte auf Verlangen des Betroffenen eine, dem Betroffenen nahe stehende Person nicht nur anwesend sein, sondern auch die Möglichkeit zur Äußerung gegenüber dem Gericht haben. Das Verlangen stand unter dem Vorbehalt, dass die Äußerung ,obne erbebliche Veræögerung möglich ist. "S 279 Abs. 3 FamFG entspricht \68a S. 4 FGG, wurde im Wortlaut jedoch insofern geändert, dass die Person nunmehr ,anzubören “597 ist. Diese Änderung soll redaktioneller Art sein ${ }^{598}$ und daher inhaltlich ohne Auswirkung. Diese Regelung könnte die für das Gericht obligatorische Hinzuziehung eines Dritten auf Wunsch des Betroffenen bei seiner Anhörung durch seine Betreuungsverfügung ermöglichen. Auch durch \68a FGG sollte eine Stärkung der Betroffenenrechte bewirkt werden. ${ }^{599}$ Die Norm diente zur Konkretisierung von \ 12 FGG, dem jetzigen \26 FamFG und nach einer Meinung allein der Sachaufklärung ${ }^{600}$ nach anderer Meinung dem recht-

\footnotetext{
591 Zimmermann FamRZ 1991, 270, 272; Epple BtPrax 1993, 156, 159.

592 BT-Drucks. 11/6308 S. 320.

593 BT-Drucks. 11/6308 S. 267.

${ }^{594}$ BT-Drucks. 16/6308 S. 267.

595 MünchKommZPO/Schmidt-Recla \278 FamFG Rn. 20.

596 Zimmermann FamFG Rn. 50 f.

${ }^{597}$ In $\int 68$ a S. 3 FGG hieß es: „Auf Verlangen [...] ist einer ibm nabe stehenden Person [...] Gelegenheit zur Äußerung zu geben."

598 BT-Drucks. 11/6308 S. 267.

${ }^{599}$ BT-Drucks. 11/4528 S. 174; als reine Sachaufklärungsnorm verstehen sie Zimmermann FamRZ 1990, 1308, 1311 und Keidel/Kuntze/Winkler/Kayser \68 FGG Rn. 1. Sie begründet keine formelle Beteiligung (Bassenge/Roth \68a FGG Rn. 4).

${ }^{600} \mathrm{Keidel} /$ Kuntze/Winkler/Kayser \68 FGG Rn. 1: Demnach konkurriere \68a FGG mit \12 FGG. Diese Einschätzung erscheint fraglich, weil \68a FGG den Kreis der anzuhörenden Personen weit umschreibt. Bei dieser Auslegung fällt wohl jede Person unter einen der in
} 
lichem Gehör Dritter. ${ }^{601}$ Nach der Gesetzesbegründung wurde die Verzögerungsgrenze aufgenommen, um die Äußerungsmöglichkeit der Vertrauensperson nicht zu einem Instrument werden zu lassen, das die Verfahrensdurchführung durch den Betroffenen mit der Absicht der Verhinderung unmöglich macht. ${ }^{602}$ Daher war und ist daraus nicht die Unverbindlichkeit des Betroffenenwunsches zu folgern. Vielmehr ist der ausdrückliche Wunsch entsprechend $\ 1901$ Abs. 2 und 3 verbindlich, soweit und solange er nicht zur tatsächlichen Blockade des Verfahrens führt. ${ }^{603}$ Gleiches muss für die Frage der Anzahl der zu nennenden Vertrauenspersonen ${ }^{604}$ gelten: solange die Anhörung der vom Betroffenen gewünschten Vertrauenspersonen vom Gericht ohne erhebliche Verzögerung möglich ist, ist dieser Betroffenenwunsch verbindlich. So war nach überwiegender Meinung vom Gericht die Beziehung des Betroffenen zu der genannten Vertrauensperson nicht zu prüfen, hier ging es allein darum, dass der Betroffene sie benannt hatte. ${ }^{605}$ Bei der Betroffenenäußerung nach der ersten Anhörung hatte das Gericht zu überlegen, eine zweite Anhörung durchzuführen. ${ }^{606}$ Rein organisatorische Probleme, wie die Ermittlung der Anschrift der Vertrauensperson lassen den Wunsch des Betroffenen nicht unverbindlich werden. ${ }^{607}$ Die Hinzuziehung Dritter zur Anhörung des Betroffenen ist auf seinen Wunsch hin daher gemäß \279 Abs. 3 FamFG in der Grenze der erheblichen Verzögerung möglich.

Nach $\ 279$ Abs. 1 FamFG hat das Gericht die sonstigen Beteiligten vor der Bestellung eines Betreuers anzuhören. Die sonstigen Beteiligten sind nach der gesetzlichen Systematik und Begründung alle in \274 FamFG Genannten. ${ }^{608}$ Nach \279 Abs. 4 FamFG ist bei der Bestellung des Betreuers auch die Person

$\int 68$ a FGG genannten Gruppen. Zur entsprechenden Regelung im FamFG: MünchKommZPO/Schmidt-Recla \279 FamFG Rn. 2.

${ }^{601}$ Wenn es der Betroffene verlangt, erwächst z.B. ein Recht auf Äußerung der Betreuungsbehörde nach Bumiller/Winkler \68a FGG Rn. 1.

${ }^{602}$ Jürgens/Kröger/Marschner/Winterstein Rn. 402; Bienwald/Sonnenfeld/Hoffmann \68a FGG Rn. 22 ff.; Damrau/Zimmermann \$ 68a FGG Rn. 17.

${ }^{603}$ Damrau/Zimmermann \68a FGG Rn. 17; MünchKommBGB/Schwab \$ 1896 Rn. 183; Zimmermann FamRZ 1991, 270, 273, 274. Als erhebliche Verzögerung wird dabei der unbekannte Aufenthalt einer Person oder die Abwesenheit aufgrund einer längeren Reise angesehen (Jürgens/Kröger/Marschner/Winterstein Rn. 402). Bienwald definiert den Begriff relativ eng (Bienwald/-

Sonnenfeld/Hofmann \68a FGG Rn. 25): Das Verlangen brauche dann nicht erfüllt zu werden, wenn ein weiterer Termin nötig werden würde, obwohl keine weiteren Hindernisse erkennbar seien, die Sache jetzt zu entscheiden.

${ }^{604}$ MünchKommZPO/Scbmidt-Recla \278 FamFG Rn. 13; HK-BUR/Bauer \68a FGG Rn. 62.

${ }^{605}$ HK-BUR/Bauer $\ 68$ a FGG Rn. 61. Eine Hinzuziehung kann aus welchen Gründen auch immer geschehen (Dodegge/Roth A. Rn. 148).

606 Wenn der Betroffenen dies wünscht: Dodegge/Roth A. Rn. 148; anders: Damrau/Zimmermann $\int 68$ FGG Rn. 53.

${ }^{607}$ Eine erhebliche Verzögerung kann man im Gegensatz zum streitigen Verfahren daher nicht schon dann annehmen, wenn wegen der Erfüllung dieses Verlangens ein weiterer Termin anberaumt werden muss.

608 BT-Drucks. 16/6308 S. 267; MünchKommZPO/Schmidt-Recla 278 FamFG Rn. 5. 
seines Vertrauens Beteiligte im Verfahren. Die Anhörung des Betroffenen ist Teil der Betreuerbestellung. Die Hinzuziehung von Dritten nach \274 Abs. 4 FamFG soll nur erfolgen können, wenn sie im Interesse des Betroffenen erfolgt. Das Interesse des Betroffenen ist nach den Grundsätzen des Betreuungsrechts subjektiv zu bewerten. Ein objektiver Maßstab stünde im Widerspruch zu \ 1901 Abs. 2 und 3, der auch im Betreuungsverfahren gilt, sowie den Grundsätzen des Betreuungsrechts, die allein den Betroffenen und seine Interessen in den Mittelpunkt stellen. ${ }^{609}$ Es kommt bei $\int 274$ Abs. 4 FamFG daher allein auf den Willen des Betroffenen an. ${ }^{610}$ Ist dieser für das Vormundschaftsgericht verbindlich, steht dem Gericht auch kein Ermessen zu. Danach greift nicht mehr die in $\int 279$ Abs. 3 FamFG formulierte Grenze der erheblichen Verzögerung. Vielmehr ist dem Wunsch des Betroffenen nachzukommen und der Dritte zur Anhörung des Betroffenen hinzuziehen. Eine Grenze ist wie bei der Betreuerbestellung ${ }^{611}$ da zu ziehen, wo die Hinzuziehung Dritter auf Betroffenenwunsch die Anhörung des Betroffenen unmöglich macht.

Die umstrittene Frage, ob der Betroffene bei fehlender (vorliegender) Äußerung auf diese Möglichkeit hingewiesen werden muss, ${ }^{612}$ kann vorliegend unbehandelt bleiben. Ein diesbezüglich in der Betreuungsverfügung enthaltener Wunsch des Betroffenen wurde geäußert und ist für das Gericht verbindlich.

\section{Ausschluss Dritter}

Gegen den Willen des Betroffenen konnten nach \68 FGG nur der Sachverständige und der Verfahrenspfleger an der Anhörung teilnehmen. ${ }^{613}$ Der Widerspruch des Betroffenen gegen die Anhörung Dritter hatte gegenüber dem Verlangen des Verfahrenspflegers und/oder des Bevollmächtigten Vorrang, weil seine Intimsphäre zu schützen war. ${ }^{614} \mathrm{Nach}$ dem ersatzlos weggefallenen \68 Abs. 4 S. 1 FGG darf nach \ 278 FamFG bei der Anhörung des Betroffenen nicht mal der Sachverständige anwesend sein. Eine Regelung für den Fall des Widerspruchs durch den Betroffenen bedarf es nach der Gesetzbegründung nicht mehr, da nach $\int 274$ Abs. 4 FamFG schon die Person nicht zu beteiligen ist, deren Beteiligung nicht im - wie ausgeführten - subjektiven Interesse des Betroffenen liegt. ${ }^{615}$ Eine Beteiligung Dritter gegen den Willen des Betroffenen ist daher entgegen der gesetzlichen Begründung aber auch dann nicht möglich, wenn ,der subjektive Wille des Betroffenen seinen objektiven Interessen zuwider läuft und [...] keine erheblichen Gründe vor-

\footnotetext{
${ }^{609}$ MünchKommZPO/Schmidt-Recla \278 FamFG Rn. 13.

${ }^{610}$ MünchKommZPO/Schmidt-Recla \$279 FamFG Rn. 4.

${ }^{611}$ Vgl. S 3 A. II.: Die Ablehnung einer Person bezieht sich nicht auf den individuellen Betreuer sondern die Betreuung insgesamt.

${ }^{612}$ Verneinend: Bassenge/Roth \ 68a FGG Rn. 4; ausführlich zum Streitstand: Bienwald/Sonnenfeld/Hoffmann $₫ 68$ a FGG Rn. 11 ff.

613 Jürgens/Kröger/Marschner/Winterstein Rn. 296, 297 ; BT- Drucks.: 11/4528 S. 89.

${ }^{614}$ Bassenge/Roth \ 68a FGG Rn. 14.

615 BT-Drucks. 16/6308 S. 267.
} 
liegen, die gegen die Hinzuriehung der Verwandten sprechen." "616 \170 S. 2 GVG stützt diese Meinung, wonach das Gericht bei entsprechendem Willen des Betroffenen die Öffentlichkeit ausschließen muss. Somit muss auch derjenige von der Anhörung ausgeschlossen werden, dessen Anwesenheit der Betroffene ausdrücklich nicht wünscht. ${ }^{617}$ Wünscht der Betroffene mittels der Betreuungsverfügung, dass bestimmte Personen bei seiner Anhörung nicht anwesend sind, ist dieser Wunsch für das Betreuungsgericht verbindlich.

IV. Anhörung von Vertrauenspersonen und Ausschluss der Anhörung Dritter gem. S\$278, 274 FamFG

$\int 279$ FamFG regelt die Anhörung der sonstigen Beteiligten, der Betreuungsbehörde und des gesetzlichen Vertreters vor der Betreuerbestellung. Die Norm soll den Grundzügen des $\int 68 \mathrm{a}$ FGG entsprechen. ${ }^{618}$ Nach $\ 278$ Abs. 1 FamFG hat das Gericht die sonstigen Beteiligten, also diejenigen, die kraft Gesetz oder durch Hinzuziehung Beteiligte sind, ${ }^{619}$ anzuhören. Nach der Gesetzesformulierung hat das Gericht in dieser Frage kein Ermessen. In $\$ 274$ FamFG sind die Beteiligten des Betreuungsverfahrens positiv genannt. Der bislang in $\$$ 68a S. 3 FGG geregelte Widerspruch des Betroffenen wurde nicht aufgenommen. Nach der Gesetzesbegründung ist dieser nicht mehr nötig, da in $\ 274$ Abs. 4 Nr. 1 FamFG nur die Verwandten beteiligt werden können, deren Beteiligung im Interesse des Betroffenen steht. ${ }^{620}$ Danach kann im Interesse des Betroffenen auch jede Person seines Vertrauens beteiligt, bzw. angehört werden. Sein Widerspruch muss hingegen als Grund für fehlendes Interesse gewertet werden. Denn die in $\ 274$ Abs. 4 Nr. 1 FamFG Genannten werden nur dann beteiligt und somit zu Beteiligten i.S.v. \ 279 Abs. 1 FamFG, wenn dem der Betroffene nicht mit erheblichen Gründen widerspricht. ${ }^{621}$ Dies ergibt sich nicht aus dem Wortlaut, soll aber nach der Gesetzesbegründung als Maßstab des \68a S. 3 FGG auch für $\ 279$ Abs. 4 FamFG gelten.

Als zwingende Norm kann der Betroffene die Anhörung der Beteiligten gem. \279 FamFG nicht verhindern. Die Hinzuziehung Dritter zu seiner Anhörung kann er gem. $\int 170$ GVG und den Grundsätzen des Betreuungsrecht verbindlich bestimmen. ${ }^{622}$ Bei der Anhörung Dritter nach $\ 279$ FamFG geht es um die alleinige Anhörung Dritter.

Mit Einführung der Anhörung der Familienangehörigen nach \68a FGG wollte der Gesetzgeber die Sachverhaltsermittlung stärken und gleichzeitig die

\footnotetext{
${ }^{616}$ Keidel/Engelhardt/Sternal/Budde \274 FamFG Rn. 10.

617 Zimmermann FamFG Rn. 477.

${ }^{618}$ BT-Drucks. 16/6308 S. 267.

${ }^{619}$ Diekmann BtPrax 2009, 149, 151.

${ }^{620}$ BT-Drucks. 16/6308 S. 267.

${ }^{621}$ BT-Drucks. 16/6308 S. 266.

${ }^{622}$ Siehe $₫ 4$ C. III. 1.
} 
Rechte der Angehörigen aus Art. 6 Abs. 1 GG wahren. ${ }^{623}$ Nach \68a FGG hatte das Gericht diesen Personen die Möglichkeit der Äußerung einzuräumen. ${ }^{624}$ \68a S. 3 FGG war dabei als Regel-Ausnahmeverhältnis gestaltet, was bedeutet, dass es grundsätzlich keinen Grund gibt, von der Anhörung abzusehen. ${ }^{625}$ Die Grenze bestand im Widerspruch des Betroffenen, der auf erheblichen Gründen, wie z. B. die Befürchtung, dass durch die Anhörung die Belange des Betroffenen beeinträchtigt sein könnten, basieren musste. Die Anforderungen an den Widerspruch waren gering. ${ }^{626} \mathrm{Da}$ dies auch für $\int 274$ Abs. 4 FamFG gelten soll, müsste daher auch hier gelten, dass ein Widerspruch des Betroffenen - aus welchen Gründen auch immer - verbindlich ist und die Anhörung einer in Frage stehenden Person unmöglich macht.

Dem Betroffenen steht nach \279 Abs. 1 i.V.m. \174 Abs. 4 S. 1 FamFG die Möglichkeit offen, eine Person seines Vertrauens durch Benennung zum Verfahrensbeteiligten zu machen, der das Gericht Gelegenheit zur Äußerung zu geben hat. Dabei kann das Gericht auch nicht nachprüfen, in welcher tatsächlichen Verbindung der Betroffene zu der genannten Person steht. ${ }^{627}$ Vielmehr soll die alleinige Nennung der Person die Pflicht des Gerichts zur Anhörung begründen. ${ }^{628}$ Nach anderer Meinung besteht die Anhörungspflicht für das Gericht auch dann nicht, wenn die Sache ansonsten entscheidungsreif ist. ${ }^{629}$ Allein entscheidend können hier nur der Wille und das Interesse des Betroffenen sein.

Demnach kann der Betroffene mittels Nennung in der Betreuungsverfügung verbindlich festlegen, welche Dritte im Verfahren durch das Gericht angehört bzw. nicht angehört werden sollen. Dies ist für das Gericht soweit verbindlich, wie es nicht die gerichtliche Sachaufklärung nach \26 FamFG (\12 FGG) verhindert, $\mathrm{zu}$ der das Gericht verpflichtet ist ${ }^{630}$. Dies wird wie bei der Benennung des Betreuers dann gegeben sein, wenn der Betroffene durch den Ausschluss aller Vertrauenspersonen die Sachaufklärung unmöglich macht. ${ }^{631}$

${ }^{623}$ BT-Drucks. 11/4528 S. 174; Jansen/Sonnenfeld \$ 68a FGG Rn. 11, 13; Bienwald/-

Sonnenfeld/Hoffmann \68a FGG Rn. 22; Damrau/Zimmermann \68a FGG Rn. 15 f.; HKBUR/Bauer \68a FGG Rn. 49.

${ }^{624}$ Jansen/Sonnenfeld \68a FGG Rn. 13; HK-BUR/Bawer \68a FGG Rn. 56; Epple BWNotZ 1992, 27, 30 und BtPrax 1993, 156, 159. Auf die Möglichkeit hinweisen soll es jedoch nicht müssen (Jansen/Sonnenfeld \$ 68a FGG Rn. 9).

625 Jansen/Sonnenfeld \$ 68a FGG Rn. 19.

${ }^{626}$ Die Rechte und Interessen auch enger Familienmitglieder an einer Beteiligung im Betreuungsverfahren müssen dann zurücktreten, wenn dies andernfalls die Belange des Betroffenen beeinträchtigt (BT-Drucks. 11/4828 S. 174).

${ }^{627}$ Auch wenn nach der Gesetzesbegründung nur solche Personen hinzuzuziehen sind, mit denen der Betroffene eng verbunden ist (BT-Drucks. 16/6308 S. 266), ist dies tatsächlich nicht nachprüfbar. Verbundenheit drückt sich nicht in tatsächlich Messbarem aus.

628 Jansen/Sonnenfeld $\int 68$ a FGG Rn. 21.

${ }^{629}$ HK-BUR/Bauer \ 68a FGG Rn. 59.

630 Zöller/Feskorn \ 26 FamFG Rn. 6.

${ }^{631} \mathrm{Vgl}$. dazu die Ausführungen unter $\$ 3$ A. II. 


\section{Sachverständigengutachten gem. $\$ \mathbb{S} 280 \mathrm{ff}$. FamFG}

$\int \ 280-284$ FamFG regeln die Einholung eines Sachverständigengutachtens im Betreuungsverfahren und sind aus $₫$ 68b FGG hervorgegangen. Nach $\ 281$ Abs. 1 FamFG ,hat vor der Bestellung eines Betreuers eine formliche Bewveisaufnabme durch Einholung eines Gutacbtens über die Notwendigkeit der Maßnabme stattzufinden. "Das Gutachten hat nach $\ 280$ Abs. 3 FamFG unter anderem das Krankheitsbild und den Zustand des Betroffenen, den Umfang des Aufgabenkreises und die voraussichtliche Dauer der Maßnahme aufzuzeigen. ${ }^{632}$ Das Gutachten wird von Gericht veranlasst und muss eine bestimmte Person bezeichnen. ${ }^{633}$ Anstelle eines Gutachtens reicht gem. $\$ 281$ FamFG in zwei Fällen ein ärztliches Zeugnis.

\section{Verweigerung oder Verzicht der Begutachtung}

Fraglich ist, ob der Betroffene sich einer solchen Begutachtung entziehen kann. Mittels in der Betreuungsverfügung enthaltener Wünsche war dies nach Meinung einiger gem. \ 68 b Abs. 1 S. 2 und S. 3 FGG möglich. ${ }^{634}$ Dies müsste auch für die jetzige gleich lautende Regelung des $\$ 281$ Abs. 1 FamFG gelten. Für diese Auslegung des Gesetzes spreche v.a., dass der Betroffene die Betreuung und somit auch die Begutachtung weitgehend mit Vollmachten umgehen könne. ${ }^{635}$

Der Betroffene kann auf ein Sachverständigengutachten nur dann verzichten, wenn die gesetzlichen Regelungen nicht zwingend sind. Nach diesen kann von der Erstellung eines Gutachtens über den Betroffenen dann abgesehen werden, wenn gem. \281 Abs. 1 Nr. 1 FamFG (\$ 68b Abs. 1 S. 2 FGG) die Bestellung des Betreuers aufgrund des Antrags des Betroffenen geschieht, dieser auf die Begutachtung verzichtet hat, ein ärztliches Zeugnis vorliegt und das Einholen eines Gutachtens - insbes. im Hinblick auf den Umfang des Aufgabenkreises des Betreuers - unverhältnismäßig wäre. Nach \281 Abs. 1 Nr. 2 FamFG (\68b Abs. 1 S. 3 FGG) kann davon abgesehen werden, wenn der Betreuer nur zur Geltendmachung der Rechte des Betroffenen gegenüber dem Bevollmächtigten bestellt wird und ein ärztliches Gutachten nach \282 Abs. 1 FamFG vorliegt $(\mathbb{S} 68 \mathrm{~b}$ Abs. 1a S. 1 FGG) und verwendet werden kann. Nach der gesetzlichen Formulierung kann das Gericht jedoch auch in diesem Fällen ein Sachverständigengutachten einholen. ${ }^{636}$

Das Gesetz schreibt mit $\ 280$ Abs. 1 FamFG die grundsätzliche Notwendigkeit der Einholung eines Sachverständigengutachtens zur Beurteilung der Betreuungsnotwendigkeit vor. Im ersten Ausnahmefall kommt der Eingriff der Betreu-

\footnotetext{
${ }^{632}$ Schon nach $\ 68 \mathrm{~b}$ FGG sollten so Grund, Art, Umfang und Dauer der notwendigen Betreuung aufgezeigt werden (Bienwald/Sonnenfeld/Hoffmann Vorbem. v. \$S 65 ff. FGG Rn. 5).

633 Jansen/Sonnenfeld \68b FGG Rn. 17; Damrau/Zimmermann \68b FGG Rn. 7.

${ }^{634}$ Epple BWNotZ 1992, 27, 30; Schmidt/Böcker Rn. 113.

${ }^{635}$ Epple BWNotZ 1992, 27, 30.

636 Ob bei Vorliegen einer Ausnahme ein zusätzliches Sachverständigengutachten eingeholt wird, liegt demnach im Ermessen des Gerichts (BT-Drucks. 15/2495 S. 41).
} 
erbestellung nicht von außen, sondern auf Anregung des Betroffenen selbst. Im zweiten erhält der Betreuer „nur“ die Aufgabe der Überwachung und soll lediglich im Zweifelsfall die Rechte des Betroffenen gegenüber dessen Bevollmächtigten geltend machen. Der Handlungsspielraum des Betreuers ist hier somit auf die Sicherung der Betroffenenrechte begrenz $\mathrm{t}^{637}$. Im dritten Fall wird auf das Gutachten nicht verzichtet. Es liegt bereits vor. Mit Einführung der letzten Ausnahme in $\int 68 \mathrm{~b}$ Abs. 1a S. 1 FGG sollten Doppelbegutachtungen vermieden werden. ${ }^{638}$ Die Fälle verbindet, dass der Eingriff in die Rechte des Betroffenen durch die Begutachtung relativ gering ist, weil diese entweder auf Anregung und daher mit Einwilligung des Betroffenen erfolgt oder bereits vorliegt. Im Hinblick auf die Möglichkeit eines Geschäftsunfähigen, die Betreuung anzuregen, ist bemerkenswert, dass allein die Tatsache der Antragstellung ausreichen soll, auf ein Sachverständigengutachten zu verzichten. ${ }^{639}$ Es bestehen Zweifel, ob dieser Betroffene die Tragweite seiner Entscheidung überblicken kann und in dieser Folge das Gericht auf ein Gutachten verzichten sollte. ${ }^{640}$

Diese gesetzliche Regelung ist v.a. mit Blick auf den Zweck des grundsätzlichen Erfordernisses eines Sachverständigengutachtens zum Schutz des Betroffenen erstaunlich. Der Betroffene dürfte aus der zugunsten des Gerichts und der Staatskasse getroffenen Entscheidung nach \281 FamFG nicht den Anspruch herleiten, von einer Begutachtung unbehelligt zu bleiben. ${ }^{641}$ Diesen Anspruch kann er nicht geltend machen, obwohl bereits die Begutachtung ein Eingriff in die Rechte des Betroffenen darstellt. Aus diesem Grund steht dem Gericht nach $\ 283$ und \284 FamFG die Möglichkeit zur Verfügung, die Vorführung des Betroffenen zur Untersuchung anzuordnen, wenn dieser nicht freiwillig erscheint. Dies kann auch gegen den Willen des Betroffenen erfolgen ${ }^{642}$ und die Vorführung ggf. unter Anwendung von unmittelbarem Zwang erfolgen. Gem. \283 Abs. 3 FamFG darf dazu sogar die Wohnung des Betroffenen ohne dessen Einwilligung betreten werden, wenn dies das Gericht aufgrund einer ausdrücklichen Entscheidung anordnet. Nach Anhörung eines Sachverständigen ist es gem. \284 FamFG schließlich möglich, den Betroffenen zur Vorbereitung der Begutachtung auf bestimmte Dauer unterzubringen. Durch diese Entscheidungen wird massiv in die Rechte des Betroffenen eingegriffen. Die Möglichkeiten, die das Gesetz dem Gericht bietet, den Betroffenen sachverständig untersuchen zu lassen, rechtfertigten

\footnotetext{
${ }^{637}$ Lt. BT-Drucks. 11/4528 S. 174 hat der Betreuer in diesem Fall eine ausschließliche Kontrollfunktion und greife so überhaupt nicht in die Rechte des Betroffenen ein.

${ }^{638}$ Das Verfahren sollte dadurch entlastet werden (BT-Drucks. 15/2495 S. 42). Nach $₫ 282$ Abs. 4 FamFG kann das Gericht nach der neuen Regelung sogar von der Einholung eines Gutachtens insgesamt absehen, wenn die übrigen Voraussetzungen für die Bestellung eines Betreuers zur Überzeugung des Gerichts feststehen.

${ }^{639}$ Vgl. \ 281 Abs. 1 Nr. 1 FamFG/ \68b Abs. 1 S. 2 FGG.

${ }^{640}$ Dazu: Bienwald/Sonnefeld/Hoffmann \68b FGG Rn. 55.

${ }^{641}$ Bienwald/Sonnefeld/Hoffmann \68b FGG Rn. 56.

${ }^{642}$ Dies sagt ausdrücklich die Gesetzesbegründung (BT-Drucks. 16/6308 S. 268).
} 
sich ausschließlich darin, dass sie zu seinem Schutz erfolgen. Dabei ist der Eingriff durch die ärztliche Begutachtung gegen den Eingriff durch die Betreuerbestellung abzuwägen. Letzteres bedeutet einen größeren und andauernden Eingriff in seine Rechte als die Erstellung eines Gutachtens. ${ }^{643}$ Daher ist sie als verhältnismäßig und somit gerechtfertigt zu bewerten. Nur so kann das verfassungsrechtlich begründete Prinzip beachtet werden, wonach der Wille des Betroffenen grundsätzlich zu beachten ist und seine Nichtbeachtung gerechtfertigt werden muss. ${ }^{644}$

Die ärztliche Begutachtung durch die Bestellung eines Bevollmächtigten zu umgehen, führt nicht zur Auslegung der \280- - 282 FamFG als dipositives Recht. Dies ist davon losgelöst zu betrachten. Die Erstellung eines Gutachtens ist vielmehr in \280 Abs. 1 FamFG gesetzlich zwingend festgeschrieben. Das Gesetz sieht nur wenige Ausnahmen vor. Die Begutachtung kann durch den Betroffenen daher nicht mittels der Betreuungsverfügung verhindert werden.

\section{Auswahl und Ausschluss des Gutachters}

Die Auswahl des Sachverständigen trifft das Gericht ${ }^{645}$ nach pflichtgemäßem Ermessen $^{646}$. Strittig ist, ob der Betroffene auf die Auswahl des Sachverständigen Einfluss nehmen und sogar die Person des Begutachters bestimmen kann.

Einige verneinen dies. ${ }^{647}$ Nach der Regel des \ 404 Abs. 1 S. 1 ZPO für den Beweis durch Sachverständige, die gem. \29 Abs. 2 FamFG ( $\int 15$ FGG) anzuwenden ist, sei ein Einfluss der Parteien nicht möglich. ${ }^{648}$ Dies gelte auch für das Betreuungsverfahren. Zudem erfolge die Auswahl des Sachverständigen als Teil des Beweisbeschlusses. ${ }^{649}$ Bei diesem habe das Gericht ausdrücklich keine Pflicht, den Betroffenen, Bevollmächtigten oder Verfahrenspfleger zur Auswahl des Sachverständigen anzuhören. ${ }^{650}$ Wegen \406 ZPO sei dies nicht nötig, wenngleich zweckmäßig. ${ }^{651}$ Die Möglichkeit der Einflussnahme des Betroffenen könne auch deshalb nicht angenommen werden, weil an den Willen des Betroffenen bzgl. der

\footnotetext{
643 Obwohl der Betreuer das Selbstbestimmungsrecht des Betroffenen wahren soll, bedeutet Betreuung immer auch ein gewisses $\mathrm{Maß}$ an Fremdbestimmung, vgl. \ $2 \mathrm{C}$.

$644 \mathrm{Vgl}$. die Ausführungen zu \2 A.

${ }^{645}$ Keidel/Kuntze/Winkle/Kayser \68b FGG Rn. 7; Jansen/Sonnenfeld \68b FGG Rn. 17; HKBUR/Rink 』 68b FGG Rn. 21; Vennemann BtPrax 1994, 93 f.

${ }^{646}$ Keidel/Kuntze/Winkle/Kayser \ 68b FGG Rn. 7; Bienwald/Sonnefeld/Hoffmann \68b FGG Rn. 16, 47; Jansen/Sonnenfeld S 68b FGG Rn. 17.

${ }^{647}$ Jansen/Sonnenfeld S 68b FGG Rn. 17: Untersagt nicht ausdrücklich die Auswahl durch den Betroffenen, bestimmt aber positiv die Auswahl durch das Gericht. KG FamRZ 1995, 1379; OLG Stuttgart FamRZ 1993, 1365.

${ }^{648} \mathrm{HK}-\mathrm{BUR} / \mathrm{R}$ ink $\int \mathrm{6}$ b FGG Rn. 21.

649 Thomas/Putz/Reichold \$ 404 ZPO Rn. 1.

${ }^{650} \mathrm{HK}-\mathrm{BUR} / \mathrm{R}$ ink $\int \mathrm{6}$ b FGG Rn. 21.

651 Thomas/Putz/Reichold \$ 404 ZPO Rn. 1.
} 
Auswahl des Gutachters keine Bindung bestehe. ${ }^{652}$ Wünschen kann das Gericht lediglich nachkommen. ${ }^{653}$

Nach anderer Meinung kann der Betroffene mittels der Betreuungsverfügung einen geeigneten Sachverständigen verbindlich vorschlagen. ${ }^{654}$ Dieser Einfluss des Betroffenen auf das Verfahren ist aus verschiedenen Gründen gerechtfertigt.

Der ausgewählte Sachverständige soll mit seinem Gutachten die Prüfung untersuchen, ob die Voraussetzungen der Betreuung aus medizinischer Sicht gegeben sind. Um ein solches Urteil abgeben zu können, muss er zur Sachverhaltsermittlung entsprechend geeignet sein. Das Gesetz bestimmt in $\int 280$ Abs. 1 S. 2, dass der Sachverständige Arzt für Psychiatrie oder Arzt mit Erfahrung auf dem Gebiet der Psychiatrie sein soll. ${ }^{655}$ Die Auswahl des Betroffenen ist also insoweit begrenzt, als es sich um einen geeigneten Sachverständigen handeln muss. Darüber hinaus lässt sich die Begrenzung des Betroffenenvorschlags nicht begründen.

Nach den oben entwickelten Grundsätzen ist der rechtlich anerkannte Wunsch des Betroffenen verbindlich. ${ }^{656}$ Dies gilt auch für das Verfahren der Sachverständigenbenennung. Ein verbindliches Vorschlagsrecht entspricht auch \26 FamFG i.V.m. $\int 404$ Abs. 3 und Abs. 4 ZPO. Nach Abs. 3 kann das Gericht die Parteien auffordern, Personen zu bezeichnen, die geeignet sind, als Sachverständige vernommen zu werden. Nach Abs. 4 hat das Gericht den Sachverständigen zu ernennen, auf den sich die Parteien geeinigt haben, es kann dann lediglich die Wahl der Sachverständigen auf eine bestimmte Zahl beschränken. Durch die FGGReform wurden allgemein Bezeichnungen und Verfahrensabläufe dem streitigen Verfahren angeglichen. Zur Vereinheitlichung wird im Rahmen des FamFG bei Möglichkeit auf die ZPO verwiesen. Daher gelten die Vorschriften der ZPO über den Beweis durch Sachverständige im FamFG entsprechend. ${ }^{657}$ Eine entsprechende Anwendung erfordert keine schematische Übertragung aller Beweisregelungen und -grundsätze, sondern soll Spielraum im Einzelfall lassen. ${ }^{658}$ Nach $\int 404$ Abs. 3 und Abs. 4 ZPO hat das Gericht demnach bereits den durch den Betroffenen genannten Sachverständigen zu benennen.

${ }^{652} \mathrm{HK}-\mathrm{BUR} / \mathrm{Rink} \int 68 \mathrm{~b}$ FGG Rn. 21. In Bezug auf die Bindung an den Betroffenenwunsch nicht eindeutig Stellung nehmend: Schmidt/Böcker Rn. 113 ,Sollte nachgekommen werden“.

${ }^{653}$ HK-BUR/Rink S 68b FGG Rn. 21; anders hingegen Schmidt/Böcker Rn. 113: Wünschen sollte nachgekommen werden, außer es bestehen Zweifel an der Unparteilichkeit.

${ }^{654}$ Epple BWNotZ 1992, 27, 30; Schmidt/Böcker Rn. 113; ohne Begründung Zimmermann Rn. 369.

${ }^{655}$ Durch die mit dem FamFG eingeführte, ausdrücklich definierte Anforderung an den Arzt, dürfte die vormals bestehende Diskussion zumindest in Bezug auf seine Fachrichtung beendet sein. Im Rahmen des FGG wurde allgemein gefordert, dass der Arzt aufgrund seiner Ausbildung und Erfahrung zur Einschätzung der Situation qualifiziert sein musste. Die Anforderungen waren im Einzelfall umstritten, hingen jedoch auch vom individuellen Fall ab (Jansen/Sonnenfeld \$ 68b FGG Rn. 14; Rink R \& P 1991, 148, 151). Der Umfang seiner notwendigen Erfahrung ergibt sich nicht aus dem FamFG und wurde auch in der Gesetzesbegründung nicht genannt.

${ }^{656} \mathrm{Vgl}$. dazu \2 C.

${ }^{657}$ Dies war nach dem FGG nur grundsätzlich der Fall (BT-Drucks. 16/8308 S. 268).

658 BT-Drucks. 16/8308 S. 268. 
Nach \406 ZPO kann der Betroffene den Gutachter zudem aus denselben Gründen wie einen Richter ablehnen. ${ }^{659}$ Bei Befangenheit oder Umständen die geeignet sind, Misstrauen gegen die Unparteilichkeit zu wecken, besteht für ihn somit die Möglichkeit, den bestimmten Sachverständigengutachter abzulehnen. Was nach der Ernennung des Gutachters gilt, muss auch für den Zeitraum vor seiner Benennung gelten. Daher kann der Betroffene einen bestimmten Gutachter auch durch antizipierte Äußerung ablehnen.

Dem positiven Vorschlag ist dieselbe rechtliche Bedeutung wie einer Ablehnung zuzumessen. Diese Einflussnahme des Betroffenen steht im Einklang mit den Zielen der 2. Reform des Betreuungsrechts, welche seine verfahrensmäßige Position stärken sollte. Seine Entscheidungskompetenz ergibt sich auch aus der Stellung des Betroffenen als Beteiligter des Betreuungsverfahrens aus $\ 274$ Abs. 1 Nr. 1 FamFG ( $\iint 68$ b Abs. 1 S. 1, 70 Abs. 1 FGG) $)^{660}$. Bedeutend ist, dass das Gesetz eine Benennung und Ablehnung eines Sachverständigen nicht verbietet. Es entspricht dem Selbstbestimmungsrecht des Betroffenen, wenn er die Auswahl des sachverständigen Gutachters alleine bestimmt. Der Betroffene kann somit in seiner Betreuungsverfügung einen Sachverständigen benennen. Dieser Vorschlag ist bei Geeignetheit des Sachverständigen für das Betreuungsgericht verbindlich. Schließt der Betroffene einzelne Personen als Gutachter aus, ist dieser Wunsch wie bei der Betreuerbestellung ${ }^{661}$ solange für das Gericht verbindlich, wie sie die Begutachtung nicht unmöglich macht, weil der Betroffene alle Geeigneten ausschließt.

\section{E. Gerichtliche Zuständigkeit}

Im Verfahren der Betreuerbestellung kann der Betroffene auf die örtliche, sachliche und funktionale Zuständigkeit des Betroffenen nur bedingt einwirken. Hier finden die allgemeinen Regelungen Anwendung. Sachlich sind gem. \23a Abs. 1 Nr. 2, und Abs. 2 Nr. 1 GVG ( $\$ 35$ FGG) die für Angelegenheiten der freiwilligen Gerichtsbarkeit zuständigen Amtsgerichte zuständig. Gem. \23c GVG werden an den Amtsgerichten Abteilungen für Betreuungssachen, Unterbringungssachen und betreuungsgerichtliche Zuweisungssachen gebildet. ${ }^{662}$ Örtlich ist nach \2 FamFG ( $\$ 36$ FGG) in dieser Reihenfolge das Gericht zuständig, 1. bei dem die Betreuung anhängig ist, wenn bereits ein Betreuer bestellt ist, 2. in dessen Bezirk der Betroffene seinen gewöhnlichen Aufenthalt hat, 3. in dessen Bezirk das Bedürfnis der

\footnotetext{
${ }^{659}$ So z.B. wenn der Gutachter eigenmächtig vom Gutachtenauftrag abweicht, vgl. Bienwald/Sonnefeld/Hoffmann \68b FGG Rn. 51; Jansen/Sonnenfeld \68b FGG Rn. 19; KG FamRZ 1995, 1379, 1380; OLG München NJW 1992, 1569.

${ }^{660}$ Lipp FS Bienwald S. 177, 186.

${ }^{661}$ Vgl. $\$ 3$ A. II.: Die Ablehnung einer Person bezieht sich nicht auf den individuellen Betreuer sondern die Betreuung insgesamt.

${ }^{662}$ Vgl. dazu Fn. 4.
} 
Fürsorge hervortritt und 4. das Amtsgericht Schöneberg in Berlin, wenn der Betroffene Deutscher ist. ${ }^{663}$ Auf die sachliche und örtliche Zuständigkeit gewährt das Gesetz dem Betroffenen keinen Einfluss. ${ }^{664}$

Die Aufteilung der funktionalen Zuständigkeit ist in $\ 15$ Rechtspflegergesetz (RPflG) zwingend geregelt und daher ebenfalls dem Einfluss des Betroffenen entzogen. 665

\section{F. Gefahr in Verzug gem. $\mathbb{S} 301 \mathrm{f}$. FamFG}

Bei Gefahr in Verzug kann das Gericht gem. \301 FamFG (\$ 69f Abs. 1 S. 5 FGG) abweichend von $\ 1897$ Abs. 4 und 5, also ohne Rücksicht auf den Betroffenenvorschlag, einen vorläufigen Betreuer bestellen. 666 Das Gericht darf wegen der besonderen Eile ausnahmsweise von aufwendigen Ermittlungen absehen. ${ }^{667}$ Sind diese nicht erforderlich, weil offenkundig, sind die Auswahlkriterien des $₫ 1897$ Abs. 4 und 5 auch bei der einstweiligen Auswahl zu beachten. ${ }^{668}$ Eine andere Auslegung von $\ 301$ Abs. 2 FamFG ist ggü. dem Eingriff in die Betroffenenrechte durch die Betreuerbestellung nicht zu rechtfertigen. Die Entbindung von $\ 1897$ Abs. 4 und Abs. 5 rechtfertigt sich allein in der Eilbedürftigkeit der Maßnahme und der darin begründeten fehlenden Zeit zur Ermittlung und Beteiligung der Genannten.

Die Vorschläge des Betroffenen sind schließlich ebenfalls zwingend bei der endgültigen Betreuerbestellung zu berücksichtigen, ${ }^{669}$ auch wenn dies nicht ausdrücklich durch $₫ 69$ Abs. 1 S. 5 FGG vorgeschrieben wird. ${ }^{670} \mathrm{Ihr}$ Nichtbeachten würde eine Umgehung der gesetzlichen Vorgaben und ein Abweichen von den betreuungsrechtlichen Zielen bedeuten.

\footnotetext{
${ }^{663}$ Bei der einstweiligen Anordnung nach $\ 300$ FamFG oder einer vorläufigen Maßregel ist auch das Gericht zuständig, in dessen Bezirk das Bedürfnis der Fürsorge hervortritt, $\ 272$ Abs. 2 S. 1 FamFG.

${ }^{664}$ Zimmermann FamFG Rn. 9.

${ }^{665}$ Näheres bei HK-BUR/Bauer $\ 1896$ Rn. 4 ff.

666 Bienwald/Sonnenfeld/Hoffmann \1897 Rn. 52.

667 Jansen/Sonnenfeld \$ 69f FGG Rn. 14.

668 Jansen/Sonnenfeld $\$$ 69f FGG Rn. 14.

${ }^{669}$ BayObLG FamRZ 1994, 1270; BayObLG FamRZ 1993, 720; abzulehnen daher BayObLG BtPrax 2004, 111.

670 So jedoch das BayObLG BtPrax 2004, 111 mit Blick auf $\int$ 69f FGG Abs. 1 S. 5, das die unverzügliche Nachholung der Verfahrensverhandlungen verlangt.
} 


\section{G. Ergebnis zu $\$ 4$}

Die Untersuchung zeigt, dass der Betroffene auch auf das Verfahren der Betreuerbestellung durch individuell formulierte, rechtlich verbindliche Wünsche einwirken kann. Er kann sowohl die Person des Verfahrenspflegers auswählen, als auch bestimmte Personen von dieser Aufgabe ausschließen. Er kann die Verfahrenspflegschaft aber nur durch alternative, d.h. privatautonome Beauftragung eines Verfahrensvertreters vermeiden. Ist er vermögend, erlaubt die gesetzliche Regelung eine individuell bestimmte Vergütung des VerfahrenspflegersDie Anhörung kann der Betroffene nicht vermeiden, ihren Ort nur vorschlagen. Die Ortswahl liegt im pflichtgemäßen Ermessen des Gerichts. Dritte können auf Wunsch des Betroffenen hinzugezogen bzw. ausgeschlossen werden. Die dadurch drohende erhebliche Verfahrensverzögerung bildet nach den Regelungen des FamFG keine Grenze für die Verbindlichkeit dieser Betroffenenwünsche mehr. Diese entsteht erst, wenn durch die Wünsche die Einrichtung der Betreuung unmöglich wird.

Das Sachverständigengutachten kann nicht aufgrund des Wunsches des Betroffenen unterbleiben, er kann aber die Person des Gutachters verbindlich vorschlagen und andere ablehnen. Letzteres wiederum nur in der Grenze, die eine Begutachtung nicht unmöglich macht.

Die gerichtliche Zuständigkeit und die einstweilige Betreuerbestellung sind hingegen gesetzlich zwingend geregelt und somit dem Einfluss des Betroffenen entzogen. 


\section{\5 Einfluss der Betreuungsverfügung auf die Betreuung gem. SS $1901 \mathrm{ff}$.}

Fraglich ist, wie der Einzelne auf die Führung der eigenen zukünftigen Betreuung mittels der Betreuungsverfügung Einfluss ausüben kann. Das Gesetz bestimmt in IS 1901 ff. die Pflichten des Betreuers. Zu untersuchen ist, ob und in welchem Umfang diese Regelungen dem Betroffenen eine individuelle Ausgestaltung seiner Betreuung erlauben. Kann der Einzelne in seiner Betreuungsverfügung festlegen, in welcher Art und Weise, nach welchen konkreten Maßstäben und Kriterien seine Betreuung geführt werden soll? Kann er die Vergütung seines Betreuers trotz der gesetzlichen Vorgaben individuell bemessen?

\section{A. Führung der Betreuung}

Nach \1901 Abs. 3 S. 1 hat der Betreuer den Wünschen des Betreuten zu entsprechen. Dies gilt nach S. 2 auch für Wünsche, die der Betroffene vor der Bestellung der Betreuung geäußert hat. Um solche handelt es sich bei der Betreuungsverfügung. Somit kann der Betroffene mit Hilfe der Betreuungsverfügung die Art und Weise, wie die Betreuung in ihren einzelnen Aufgaben geführt werden soll, 
bestimmen. ${ }^{671}$ Trotz dieser gesetzlichen Formulierung erblicken einige, die den Wünschen des Betreuten keine verbindliche Wirkung sondern Richtliniencharakter zusprechen, in der Betreuungsverfügung keine so weit reichende Regelungsmöglichkeit. ${ }^{672}$ Sie beschränken die tatsächliche Wirkung auf die Nennung des Wunschbetreuers und die Formulierung von Wünschen. Deren Bindung beschreiben sie als gering und beschränken sie auf wenige Bereiche. ${ }^{673}$ Vertreter der weiten Bindungswirkung antizipierter Wünsche in Form der Betreuungsverfügung setzen den Regelungsinhalt zum Teil mit dem der Vorsorgevollmacht gleich ${ }^{674}$. Die konkrete Frage, was genau der Einzelne in der Betreuungsverfügung in Bezug auf die (Aus-)Führung der Betreuung regeln kann, ist bislang nicht umfassend beantwortet. Nach dem dieser Arbeit zugrunde liegenden Verständnis des Rechts auf Selbstbestimmung kann der Einzelne mit Blick auf die gesetzlichen Formulie-

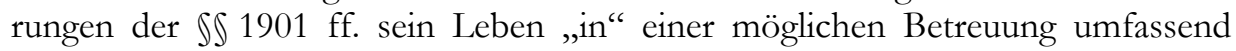
regeln. Nur die zwingenden betreuungsrechtlichen Regelungen begrenzen die individuelle Ausgestaltungsfreiheit der eigenen Betreuung. Welches diese sind, ist zu untersuchen.

I. Pflichten des Betreuers gem. \1901

$\int 1901$ Abs. 2 bestimmt: „Der Betreuer hat die Angelegenheiten des Betreuten so zu besorgen, wie es dessen Wobl entspricht. Zum Wobl [...] gebört auch [...] sein Leben nach seinen eigenen Wünschen und Vorstellungen zu gestalten. "Dies gilt nach Abs. 3 S. 2 ,auch für Wünsche, die der Betreute vor der Bestellung des Betreuers geäußert hat". Zu untersuchen ist, was diese Wünsche beinhalten können.

\section{Aufgabenkreis und persönliche Betreuung}

Aus der Aufgabe der Betreuung, die rechtliche Handlungsfähigkeit des Einzelnen herzustellen und seine Rechtsgleichheit zu verwirklichen, ist abzuleiten, dass sich der Aufgabenbereich nur auf diese Aufgabe der Rechtsfürsorge beziehen kann. In dieser Aufgabe findet die Betreuung ihre Grenzen. ${ }^{675}$ Ein antizipiert geäußerter Wunsch des Betreuten darf von dem Betreuer nur beachtet werden, wenn er in dessen Aufgabenbereich fällt. ${ }^{676}$

Nach \1901 Abs. 1 muss der Betreuer die Aufgaben rechtlich besorgen und den Betroffenem ,hierfür“ im erforderlichen Umfang persönlich betreuen. Der Wortlaut macht die persönliche Betreuung als Instrument zur Umsetzung der rechtlichen Angelegenheiten deutlich. Soweit die Umsetzung der rechtlichen An-

\footnotetext{
${ }^{671}$ So sehen das auch Bübler/Kren/Stolz S. 22 f.; Lipp FS Bienwald S. 177, 179 ff.; Winkler S. 60, 63 f.;

Frost S. 49; Palandt/Diederichsen Einf. v. \1896 Rn. 8; Zimmermann Rn. 368-370.

${ }^{672} \mathrm{HK}-\mathrm{BUR} /$ Baner $\int 1901 \mathrm{Rn} .42 \mathrm{ff}$.

673 So etwa: HK-BUR/Bauer $\int 1901$ Rn. 49 ff.

674 Coeppicus Sachfragen S. 51 (C.).

675 Lipp S. 153.

${ }^{676}$ Knittel S 1901 Rn. 8.
} 
gelegenheiten dies fordert, ist eine persönliche Betreuung erforderlich. ${ }^{677}$ Diese erfordert zumeist einen direkten Kontakt zwischen Betreuer und Betroffenen, ${ }^{678}$ welcher je nach Aufgabenbereich unterschiedlich ist. ${ }^{679}$ Gem. \ 1901 Abs. 2 und 3 bestimmen das Wohl und die Wünsche des Betreuten das Betreuerhandeln. 680 Eine konkrete Wohlgefährdung durch den Betreuer durch fehlende persönliche Betreuung macht diesen ungeeignet für die Ausübung des Amts. ${ }^{681}$ Das Betreuungsgericht muss sich bei der Betreuerauswahl maßgeblich davon leiten lassen, durch wen die bestmögliche Kombination von aufgabenbezogener persönlicher Betreuung bei Besorgung der rechtlichen Angelegenheiten des Betreuten gewährleistet wird. ${ }^{62}$ Auf diese Grundsätze des Betreuungsrechts kann der Betroffene nicht mittels individuell abweichender Entscheidung einwirken.

\section{Wünsche und Vorstellungen des Betreuten}

Äußert der Betroffene eigene Wünsche und Entscheidungen, trifft er dadurch antizipiert eine eigene Entscheidung. Die Aufgabe des Betreuers besteht dann darin, die Entscheidung des Betreuten herbeizuführen.

Bei der Wirkung der Wünsche ist zwischen Innen- und dem Außenverhältnis zu unterscheiden. Im Innenverhältnis, der Beziehung zwischen Betreutem und Betreuer sind die Wünsche des Betreuten verbindlich. Außenwirkung entfalten sie jedoch erst, wenn der Betreuer sie umsetzt. Konkret formulierten Wünschen hat der Betreuer dabei gem. \1901 Abs. 3 S. 1 zu entsprechen, sie also direkt umzusetzen und zu verwirklichen. Er muss sie vorher aber auch kontrollieren und ihr ggf. die Anerkennung versagen. ${ }^{683}$ Die Kontrolle bezieht sich dabei v.a. auch auf die Frage, ob das Betreuungsrechts für den Betreuten die individuelle Einflussnahme mittels seines Wunsches vorsieht und zulässt.

\footnotetext{
677 Die rechtliche Umsetzung der personenbezogenen Angelegenheiten erfordert beispielsweise eine intensivere persönliche Beziehung als bei sachbezogenen Angelegenheiten. Bei Fragen im Aufgabenkreis der Gesundheitssorge wird die Intensität einer persönlichen Betreuung grundsätzlich hoch, im Fall der Vermögenssorge eher geringer sein.

${ }^{678}$ Räumliche Entfernung steht dem grundsätzlich entgegen Palandt/Diederichsen \1897 Rn. 4; Staudinger/Bienwald \$ 1897 Rn. 15; BT-Drucks. 11/4528 S. 125, 68; Dodegge/Roth B. Rn. 35 f.; Coeppicus BtPrax 2003, 164, 165; OLG Köln FamRZ 1996, 506.

${ }^{679}$ Die Betreuung kann ausnahmsweise in großer Entfernung zum Betroffenen ausgeübt werden (Staudinger/Bienwald \$ 1897 Rn. 15; Knittel\$ 1897 Rn. 10a; OLG Köln FamRZ 1996, 506). Zur persönlichen Betreuung ist z.B. keine körperliche Pflege erforderlich, sondern vielmehr Zeit und Geduld zur persönlichen Beziehung- und Kontaktaufnahme zum Zweck der Kommunikation (Palandt/Diederichsen \$1897 Rn. 4; Dodegge/Roth B. Rn. 38). Daher muss der Betreuer in diesem Fall die Fähigkeit zum Umgang mit krankheits- oder behinderungsbedingten Verhaltensweisen besitzen, um adäquat agieren zu können (Staudinger/Bienwald \$1897 Rn. 15).

${ }^{680}$ KG Berlin FamRZ 2006, 889, 890; Staudinger/Bienwald \ 1897 Rn. 15; Dodegge/Roth B. Rn. 38.

${ }^{681}$ Staudinger/Bienwald \$ 1897 Rn. 10.

${ }^{682}$ Staudinger/Bienwald \ 1897 Rn. 16, vgl. dazu auch \3 B.

${ }^{683}$ Lipp S. 155.
} 
Allgemeine Wünsche und Vorstellungen des Betreuten hat der Betreuer hingegen gem. \1901 Abs. 2 zu beachten und anhand dieser Vorgaben die Angelegenheiten des Betreuten zu besorgen. Seine Entscheidung muss dabei an den Wünschen und Vorstellungen des Betreuten ausgerichtet sein. Er muss die Entscheidung des Betreuten herbeiführen.

II. Medizinische Behandlung gem. SS 1901a, 1901b

Durch das 3. Gesetz zur Änderung des Betreuungsrechts (3. BtÄndG) ${ }^{684}$ wurde die Verbindlichkeit der Betroffenenentscheidung über medizinische Behandlungen positiv in $\iint 1901 \mathrm{a}$ und 1901b geregelt. ${ }^{685}$ Das Gesetz trat zum 01.09.2009 in Kraft und fügte die $\int \$ 1901$ a, 1901b, 1904 Abs. 2 bis 4 in das BGB ein. ${ }^{686}$ Der bisherige $\ 1901$ a wird zu \ 1901c. \1901a Abs. 1 enthält nun die Legaldefinition der Patientenverfügung: Darunter ist die schriftliche Festlegung des einwilligungsfähigen Volljährigen zu verstehen, ob er für den Fall seiner Einwilligungsunfähigkeit in bestimmte, zum Zeitpunkt der Festlegung noch nicht unmittelbar bevorstehenden Untersuchungen seines Gesundheitszustandes, Heilbehandlungen oder ärztliche Eingriffe einwilligt oder diese untersagt. Die Verbindlichkeit dieser antizipierten Entscheidungen ist nunmehr uneingeschränkt anerkannt. Liegt in der Betreuung ein in der Patientenverfügung beschriebener Fall vor, hat der Betreuer dem Willen des Betreuten Ausdruck zu verleihen und Geltung zu verschaffen.

Entscheidungen über unmittelbar bevorstehende ärztliche Maßnahmen, mündliche Entscheidungen und andere Formen der Willensbekundung sind nicht vom gesetzlichen Begriff der Patientenverfügung umfasst. ${ }^{687}$ Diese Äußerungen entfalten auch nach der neuen Fassung des \1901a keine unmittelbare Rechtswirkung. Sie sind gem. \$1901a Abs. 2 S. 1 als Behandlungswünsche des Betroffenen zu verstehen und müssen als solche gem. $\ 1901$ Abs. 3 S. 2 und 3 erst durch den Betreuer konkretisiert und dann umgesetzt werden.

Bei der Feststellung der Betroffenenwünsche nach \1901a Abs. 2 soll gem. \1901b nahen Angehörigen oder sonstigen Vertrauenspersonen des Betreuten Gelegenheit zur Äußerung gegeben werden, sofern dies ohne erhebliche Verzögerung möglich ist.

Fragen zur medizinischen Behandlung kann der Betroffene somit auch im Rahmen der Betreuungsverfügung regeln. Mit Blick auf die Bindung des Betreuers an die Patientenverfügung, muss sich der Betroffene überlegen, ob er seinen späteren Betreuer wirklich verpflichten will oder ob er ihm durch formulierte Wün-

${ }^{684}$ Das 3. BtÄndG vom 29.07.2009, BGBl. I S. 2286 ff. ist am 18.06.2009 vom Bundestag angenommen worden (Gesetzentwurf: BT-Drucks. 16/8442; Beschlussempfehlung und Bericht des Rechtsausschusses: BT-Drucks. 16/13314; Beschluss des Bundestages: BR-Drucks. 503/09). ${ }^{685}$ Zur bisherigen Rechtslage z.B. Lipp/Klein FPR 2007, 56 ff.

${ }^{686}$ Des Weiteren wurde das Verfahren für die gerichtliche Genehmigung nach $\$ 1904$ in $\$ \$ 287$ Abs. 3, 298 FamFG angepasst.

687 BT-Drucks. 16/8442 S. 13. 
sche einen mehr oder weniger großen Entscheidungsspielraum lassen möchte. Festlegungen, die einer Patientenverfügung entsprechen, muss der Betroffene aufgrund des Formerfordernisses des \$1901a Abs. 1 auch im Rahmen der Betreuungsverfügung schriftlich abfassen. Diese Entscheidungen des Betroffenen hat der Betreuer im gegebenen Fall ohne eigenen Entscheidungsspielraum umzusetzen. Hält der Betroffene in der Betreuungsverfügung hingegen allgemeine Wünsche zur medizinischen Behandlung oder deren Unterlassen fest, hat der Betreuer gem. \1901a Abs. 2 i.V.m. \1901 Abs. 2 auf dieser Grundlage zu entscheiden.

Die SS 1901a und 1901b legen die Verbindlichkeit der Betroffenenwünsche in Bezug auf medizinische Fragen fest. Sie beschränken die Wünsche des Betroffenen nicht inhaltlich. Mittels der Betreuungsverfügung kann der Betroffene Wünsche über alle medizinischen Fragen äußern. Durch \$S 1901a und 1901b i.V.m. \1901 Abs. 3 ist der Betreuer nun eindeutig an diese Wünsche gebunden.

\section{Genehmigungsvorbehalte der $\int \$ 1904-1908,1908 \mathrm{i}$}

Das Gesetz nennt neben $\int \mathbb{S} 1901 \mathrm{a}, 1901 \mathrm{~b}$ und $\ 1901$ Abs. 3 keine weitere ausdrückliche Pflicht zur Beachtung der Wünsche und Vorstellungen des Betroffenen. Fraglich ist, ob die betreuungsgerichtlichen Genehmigungsvorbehalte der \\1904 bis 1908 sowie die Verweise nach $\$ 1908$ i indirekt zwingende gesetzliche Regelungen vorsehen und damit eine Einflussnahme mittels Wünschen und Vorstellungen der Betreuungsverfügung auf Einzelfragen der Führung der Betreuung verwehren.

Die gerichtlichen Genehmigungsvorbehalte des Betreuungsrechts knüpfen an die gesetzliche Vertretungsmacht des Betreuers. Dieser vertritt den Betreuten in seinem Aufgabenbereich, vgl. \1902. Dabei handelt es sich um eine gesetzliche Vertretungsmacht, ${ }^{688}$ auf die der Betreute nicht einwirken kann - auch nicht durch antizipierte oder aktuelle Wunsch- oder Vorstellungsäußerung. Gleiches gilt für die Genehmigungsvorbehalte der $\int \mathbb{S} 1904 \mathrm{ff}$. Der Betreute kann somit weder durch antizipierte Wünsche in der Betreuungsverfügung, noch durch aktuelle Wünsche in der Betreuung die Genehmigung wirksam selber erteilen ${ }^{689}$ und so ein gerichtliches Eingreifen vermeiden. Dies gilt auch, wenn der Betreute geschäftsfähig ist. ${ }^{690}$ Bei den Genehmigungsvorbehalten der $\iint 1904-1908$ handelt es sich vielmehr um zwingendes und daher unabdingbares Recht. ${ }^{691}$

Nach früher verbreiteter Ansicht kann der geschäftsfähige Betreute dem Betreuer auch in dessen Aufgabenkreis Vollmachten erteilen, die über die gesetzliche

\footnotetext{
688 Schwab FamRZ 1992, 493, 504.

689 OLG Frankfurt FamRZ 1997, 1424, 1425; BayObLG NJW-RR 1998, 158 f.; Perau MittRhNotK 1996, 285, 288; Epple BWNotZ 1992, 27, 29; Langenfeld S. 166 f. So ist wohl auch Renner zu verstehen: Müller/Renner Rn. 330 f. und Rn. 131 (1. Auflage).

690 OLG Frankfurt FamRZ 1997, 1424, 1425.

${ }^{691}$ Perau MittRhNotK 1996, 285, 288.
} 
Vertretungsmacht hinausgehen und so eine betreuungsgerichtliche Genehmigung entbehrlich machen. ${ }^{692}$ Die gegenteilige Auffassung sieht auch heute noch v.a. mit Blick auf die amtliche Begründung des Betreuungsrechts eine solche Freistellung von gesetzlich zwingenden Vorschriften nicht vor. ${ }^{693}$ Der Sinn der Genehmigung liege in der Kontrolle der Betreuerentscheidung zum Schutz des Betreuten. Dies gelte, obwohl das in der gesetzgeberischen Begründung enthaltene Ziel nicht in $\int 1902$ Ausdruck erhalten hat. Eine rein privatautonome Bevollmächtigung sei nur im Rahmen einer Vorsorgevollmacht und nicht in einer Betreuungsverfügung möglich. Wenn in einem Schriftstück einerseits eine Vollmacht erteilt, andererseits Wünsche für die Betreuung festgehalten werden, ist es jedoch beides.

Die wirksame Bevollmächtigung eines Dritten ist zumindest für die medizinische Behandlung nach \ 1904 Abs. 4 und für die Unterbringung nach \1906 Abs. 5 möglich. In beiden Fällen unterliegt auch der Bevollmächtigte dem betreuungsgerichtlichen Genehmigungsvorbehalt. In dem Aufgabenbereich, für den ein Betreuer bestellt wurde, kann der Betreute den Betreuer zwar wirksam bevollmächtigen. Die Betreuung wäre dann jedoch nicht mehr erforderlich und daher aufzuheben. Eine Bevollmächtigung, die keine Schnittmenge mit der Betreuung hat, kann aber grundsätzlich neben einer Betreuung bestehen. Deshalb kann der Betreute seinen Betreuer auch über dessen Aufgabenkreis hinaus bevollmächtigen. Nach der Gesetzesbegründung sollen die Schranken, die dem Betreuer innerhalb seines Wirkungskreises auferlegt werden, für ihn jedoch über den Aufgabenkreis hinaus gelten. ${ }^{694}$ Die Genehmigungstatbestände seien somit auch für ihn zwingend, eine Umgehung für den Betreuten durch Bevollmächtigung des Betreuers also nicht möglich.

Nach der gesetzlichen Regelung sind die vormundschaftlichen Genehmigungserfordernisse der \$S 1904 - 1908 zwingendes Recht und als solche durch den Betreuten weder mittels einer eigenen antizipierten Genehmigung in der Betreuungsverfügung noch durch Einwilligung während der Betreuung abdingbar. Anderes gilt, wenn die Person als Bevollmächtigte handelt.

$\mathrm{Zu}$ untersuchen ist daher, ob die Genehmigungstatbestände bestimmte zwingende Vorgaben für die inhaltliche Tätigkeit des Betreuers enthalten oder der allgemeine Maßstab des $₫ 1900$ Abs. 3 gilt.

\footnotetext{
${ }^{692}$ Schwab FamRZ 1992, 493, 504; Damrau/Zimmermann \1902 Rn. 3; Knittel \ 1902 Rn. 6; MünchKommBGB/Schwab \1902 Rn. 4 f.; Bienwald/Sonnenfeld/Hoffmann \1902 Rn. 34; Cypionka DNotZ 1991, 571, 577; zur früheren Gebrechlichkeitspflegschaft: RG HRR 1930, Nr. 615; KG Berlin WM 1971, 871, 872; OLG Karlsruhe FamRZ 1957, 57; Schwab FamRZ 1992, 493, 504. ${ }^{693}$ BT-Drucks. 11/4528 S. 135; OLG Köln FamRZ 2000, 1525; Palandt/Diederichsen \1902 Rn. 2; Holzhauer/Reinicke \1902 Rn. 13; Jürgens/Jürgens \1902 Rn. 8; Erman/Hol₹̧auer \1902 Rn. 13 und im Anschluss Erman/Roth \1902 Rn. 13 ff. ${ }^{694}$ BT-Drucks. 11/4528 S. 136.
} 


\section{Sterilisation des Betreuten gem. $\int 1905$}

Fraglich ist, ob das Gesetz dem Einzelnen die Möglichkeit einräumt, durch antizipierte Äußerung im Rahmen der Betreuungsverfügung trotz zwingend erforderlicher gerichtliche Genehmigung Einfluss auf die Frage einer möglicherweise erforderlichen Sterilisation zu nehmen. Der Gesetzgeber wollte mit der Schaffung von $\int 1905$ zum einen psychisch Kranke und geistig-seelisch Behinderte, bei denen eine Empfängnisverhütung auf andere Weise nicht (praktikabel) durchführbar ist und nur die Sterilisation zu einem unbeschwerten Sexualleben führt, erfassen. ${ }^{695}$ Zum anderen sollte der bisherige ungeregelte Zustand dieser Materie behoben werden. Vor Einführung der Norm durch das Betreuungsgesetz wurden zahlreiche Sterilisationen in einer rechtlichen Grauzone durchgeführt. ${ }^{696}$ \ 1905 soll dabei eine Spezialvorschrift zu \ 1904 darstellen, vgl. Abs. 1.697

Die neue Regelung hat mit ihrem restriktiven Charakter wenig Zustimmung erfahren. Bis heute ist umstritten, ob die Übertragung dieser vertreterfeindlichen Entscheidung auf einen Bevollmächtigten überhaupt verfassungskonform ist oder dies eine nicht zu rechtfertigende Verletzung der Menschenwürde und des Persönlichkeitsrechts darstellt. Nach heute herrschender Meinung sind stellvertretende Entscheidungen in dieser Frage zulässig. ${ }^{698}$ Nach langer Debatte in der Öffentlichkeit wurde die Sterilisation des Betreuten ${ }^{699}$ unter den engen Voraussetzungen des \1905 Abs. 1, durch Bestellung eines besonderen Betreuers gem. \1899 Abs. 2, unter vormundschaftlicher Kontrolle in Form der Genehmigung der Einwilligung des Betreuers nach $\int 1905$ Abs. 2 S. 1 und unter den Anforderungen an die Durchführung der Maßnahme nach Abs. 2 S. 2, 3 eingeführt. Die inhaltlichen Voraussetzungen der Sterilisation orientieren sich an der individuellen Situation des Betreuten ${ }^{700}$ und seinem Wohl. ${ }^{701}$ Daher darf nach Abs. 1 Nr. 1 die Sterilisation nicht dem Willen des Betreuten widersprechen. Widerspricht der Betreute, ist

\footnotetext{
${ }^{695}$ BT-Drucks. 11/4528 S. 73 ff.; BT-Drucks. 11/6949 S. 73 ff. - dies dürfte aufgrund der medizinischen Entwicklung im Bereich der hormonellen Verhütung mittlerweile zumindest zum Teil anders sein.

${ }^{696}$ Der Rechtsausschuss des BT schätzte 1000 Fälle pro Jahr; BT-Drucks. 11/4528 S. 74 schätzt 30000 - 50000 Frauen-Sterilisationen pro Jahr in der BRD.

${ }^{697}$ Lt. amtlicher Begr. vgl. BT-Drucks. 11/4528 S. 141; zustimmend: Knittel \$ 1905 Rn. 25; MünchKommBGB/Schwab \$1904 Rn. 3; Bienwald/Sonnenfeld/Hoffmann \1905 Rn. 1; Damrau/Zimmermann \$1905 Rn. 1; Erman/Roth \1905 Rn. 7; Mayer S. 173.

${ }^{698}$ Mayer S. 173; MünchKommBGB/Schwab \1905 Rn. 3; Pieroth FamRZ 1990, 117, 120 m.w. Ausführungen.

${ }^{699}$ Unter Sterilisation wird die gezielte permanente Unfruchtbarkeit durch einen operativen Eingriff an den Transportwegen des Eies oder der Gebärmutter verstanden (Laufs/Katzenmeier/Lipp Kapitel VII. Rn. 1; BayObLG FamRZ 1997, 702, 703 unter Berufung auf Knittel \$ 1905 Rn. 1). Entscheidend ist die dauerhafte medizinische Unfruchtbarkeit (MünchKommBGB/Schwab \1905 Rn. 1).

700 Jürgens/Kröger/Marschner/Winterstein Rn. 218; MünchKommBGB/Schwab \$1905 Rn. 12; Mayer S. 171.

${ }^{701}$ MünchKommBGB/Schwab \1905 Rn. 4.
} 
ausreichend, wenn er dazu einen natürlichen Willen bilden kann. ${ }^{702}$ Der Betreuer ist nur einwilligungsbefugt, wenn es dem Betreuten an der erforderlichen Einwilligungsfähigkeit mangelt. ${ }^{703}$ S 1905 verbietet Zwangssterilisation jeder Art.

Ein Einfluss des Einzelnen auf die Frage der Sterilisation ist mittels der Betreuungsverfügung trotz des zwingenden Charakters von \ 1905 nicht ausgeschlossen: Die engen Voraussetzungen der Sterilisation ${ }^{704}$ fordern in $\ 1905$ Abs. 1 Nr. 1, dass diese dem Willen des Betreuten nicht widerspricht. Ein Verfasser der Betreuungsverfügung kann somit durch die Formulierung seines Willens auf eine, seine Person betreffende Sterilisation Einfluss nehmen, indem er seine Ablehnung formuliert. Der in der Betreuungsverfügung enthaltene Wille ist verbindlich. Dies gilt auch für den eine Sterilisation ablehnenden Willen. Damit würde der Betroffene formulieren, dass die Sterilisation seinem Willen widerspricht, die Voraussetzungen für die Einwilligung eines Betreuers in die Sterilisation liegen dann gem. \1905 Abs. 1 Nr. 1 nicht vor. Der Betroffene kann seine Sterilisation somit mittels der Betreuungsverfügung vorsorglich wirksam verhindern.

In der Betreuung betrifft die Sterilisation in erster Linie Behinderte. ${ }^{705}$ Der Anwendungsbereich des $\int 1905$ auf die Betreuungsverfügung wird aufgrund seiner weit reichenden Wirkung dennoch groß sein.

\section{Unterbringung des Betreuten gem. $\int 1906$}

$\int 1906$ regelt die Voraussetzungen der Unterbringung und alle sonstigen freiheitsbeschränkenden sowie freiheitsentziehenden Maßnahmen, die den Betreuten betreffen. $\mathrm{Zu}$ untersuchen ist, ob der Einzelne auf diese Voraussetzungen mittels der Betreuungsverfügung einwirken kann. ${ }^{706}$ Kann er so eine zwangsweise Unterbringung in einer geschlossenen Wohnform ${ }^{707}$ verhindern; das ununterbrochene oder regelmäßige Verlassen seines Aufenthaltsortes

702 Mayer S. 178, 186: Geschäftsfähigkeit ist dabei ebenfalls irrelevant. Der Betreute ist widerspruchsfähig (Frost S. 185-91, insb. S. 188; Bienwald/Sonnenfeld/Hoffmann \ 1905 Rn. 13).

703 Mayer S. 186: Die freiwillige Sterilisation eines Einwilligungsfähigen fällt nicht in die Betreuung, da der Betroffene seine Angelegenheiten selber erledigen kann. Sie erfüllt keinen Straftatbestand, vgl. BGHSt 20, 81 ff.

704 Gem. \ 1905 kann der Betreuer nur in eine Sterilisation des Betreuten einwilligen, wenn sie dem Willen des Betroffenen nicht widerspricht, dieser auf Dauer einwilligungsunfähig bleiben wird, die Annahme besteht, dass es ohne Sterilisation zu einer Schwangerschaft kommen würde, infolge derer eine Gefahr für das Leben oder den körperlichen oder seelischen Gesundheitszustand der Schwangeren zu erwarten ist oder eine solche Schwangerschaft nicht anders verhindert werden kann.

705 Mayer S. 193; Frost S. 162; BT-Drucks. 11/4528 S. 79: Sie trifft primär Frauen, aber auch Männer, auch wenn dies bestritten wird.

706 Dies untersucht auch schon Hartmann mit Hilfe der Psychiatrischen Verfügung in NStZ 2000, 113, 117.

707 Hier ist auch der Fall zu nennen, in dem die Eingangstür zeitweilig - insbesondere nachts verschlossen wird. 
oder regelmäßige Verlassen seines Aufenthaltsortes sichern; ${ }^{708}$ die Fixierungen an dem Bett mit Bauch- und/oder Armgurten verhindern?

Der Gesetzgeber wollte mit \ 1906 die freiheitsentziehende zivilrechtliche Unterbringung des Betreuten ohne oder gegen seinen Willen regeln.709 Mit dem Genehmigungsvorbehalt des Abs. 2 sollte ein doppelter Schutz des Betreuten vor der unzulässigen freiheitsentziehenden Maßnahme geschaffen werden. ${ }^{710}$ Die Rechtsprechung war und ist in der Anwendung und der Auslegung des \ 1906 uneinheitlich. ${ }^{711}$ Auch die zahlreichen Abhandlungen zu $\int 1906$ spiegeln die juristischen Probleme mit der Handhabe von freiheitsein- und freiheitsbeschränkenden Maßnahmen (inkl. der Unterbringung) des Betreuten gegen oder ohne seinen Willen. \1906 normiert nach seinem Wortlaut keine Pflicht des Betreuers, sondern beschreibt, wann eine freiheitsentziehende Maßnahme zulässig ist. Der Betreuer entscheidet, ob die Voraussetzungen vorliegen und bedarf dann nach Abs. 2 S. 1 zur Unterbringung des Betreuten der gerichtlichen Genehmigung. Gem. Abs. 1 S. 1 ist die freiheitsentziehende Maßnahme nur zulässig, solange sie zum Wohl des Betreuten erforderlich ist. Im Sinne der Erforderlichkeit darf also keine weniger einschneidende Maßnahme ${ }^{712}$ möglich sein. Demnach ist das Wohl des Betreuten der regulierende Faktor. ${ }^{713}$

Wie oben geschildert, ${ }^{714}$ definiert derjenige, der Wünsche und Vorstellungen in einer Betreuungsverfügung formuliert, mit diesen sein individuelles Wohl. Dieses darf durch die freiheitsentziehende Maßnahme nicht beeinträchtigt werden. Folglich kann der Betroffene durch in der Betreuungsverfügung formulierte Wünsche die Unzulässigkeit von freiheitsentziehenden Maßnahmen im Sinne von \ 1906 Abs. 1 bestimmen. Ist es für das objektive Wohl des Betroffenen beispielsweise erforderlich, dass er mit einem Bettgitter schläft, hat er dieser Maßnahme aber in der Betreuungsverfügung widersprochen, muss die Zulässigkeit dieser Maßnahme wie folgt geprüft werden: Durch die antizipierte Formulieren seiner Wünsche und Vorstellungen, nicht in einem Bett mit Bettgitter schlafen zu wollen, hat er sein Wohl dahingehend formuliert, dass diesem angebrachte Bettgitter widersprechen. Das Anbringen von Bettgittern würde seinem subjektiven Wohl also nicht entsprechen. Die Maßnahme wäre daher nicht erforderlich und folglich gem. \1906

\footnotetext{
708 BT-Drucks. 11/4528 S. 146.

${ }^{709}$ BT-Drucks. 11/4528 S. 146, 148; Holz̧hauer FuR 1992, 249, 255: Willigt der Betroffene mit natürlichem Willen ein, liegt keine Unterbringung vor. Anderer Ansicht sind: MünchKommBGB/Schwab \1800 Rn. 28 und Diekmann JZ 1988, 789, 799.

710 BT-Drucks. 11/4528 S. 148.

711 Vgl. nur BayObLG FamRZ 1993,600; Düsseldorf FamRZ 1995, 118. Diesbezüglich hat das höchstrichterliche Urteil zur Frage der Zwangsbehandlung etwas Klarheit geschaffen, wenn dies auch weiterhin umstritten ist, vgl. nur Lipp BtPrax 2008, 51 ff. und JZ 2006, 661 ff.

712 BT-Drucks. 11/4528 S. 146; Wigge MedR 1996, 291, 292.

713 Anderer Ansicht ist Harm. Seiner Meinung nach ist Personensorge gegen den Willen der betreuten Person für den gesetzlichen Vertreter nicht zugänglich, außer im Falle der abschließend normierten Eingriffe des $\ 1906$ (Harm Rpfleger 1998, 89, 91).

714 Vgl. $\$ 2$ C.
} 
unzulässig. ${ }^{715}$ Der objektive Schutz, der durch die Montage von Bettgittern herbeigeführt werden sollte, z.B. dass der Betreute nicht aus dem Bett fällt, müsste dann durch andere zulässige Maßnahmen geschaffen werden..$^{716}$

Das Recht auf seine eigene Krankheit ermöglicht dem Betroffenen, sein Wohl nach seinen Wünschen und Vorstellungen zu definieren. ${ }^{717}$ Dabei kann nicht entscheidend sein, ob ein Fall der Selbstgefährdung vorliegt. Allein maßgeblich ist das subjektive Wohl des Betreuten. ${ }^{718}$ Sein eigener Wunsch steht zu diesem Wohl nicht im Widerspruch.

Als zwingendes Recht ist $\int 1906$ nicht durch den Betreuten abdingbar. Weil eine Maßnahme nach $\ 1906$ jedoch nur zulässig ist, solange sie zum Wohl des Betreuten erforderlich ist, ist jede Maßnahme an seinen Wünschen und Vorstellungen und somit an seinem subjektiven Wohl zu messen. Mittels konkreter Wünsche in der Betreuungsverfügung kann der Betroffene daher definieren, welche Maßnahmen nicht seinem Willen und folglich seinem subjektiven Wohl entsprechen. So kann er beispielsweise antizipiert bestimmen, dass die Montage von Bettgittern am Bett, das Verhindern am Verlassen des Gebäudes durch komplizierte Schließmechanismen, Pförtner oder anderes Personal oder die Ruhigstellung durch Schlafmittel oder sonstige Medikamente nicht seinen Wünschen entsprechen.

\section{3. Übrige Angelegenheiten des Betreuten}

Das Gesetz regelt in den $\int \mathbb{S} 1901$ ff. weitere Angelegenheiten des Betreuten. Thematisch hat sich die Unterscheidung in Fragen der Gesundheit (\$S 1904, 1905), des Aufenthaltes (\$\$ 1906, 1907) und des Vermögens (\$S 1908, 1908i) durchgesetzt.

a) Fragen der Gesundheit

Neben $\int \mathbb{S} 1901 \mathrm{a}, 1901 \mathrm{~b}$ und $\$ 1905$ nennt das Gesetz nur noch in $\$ 1904$ Regelungen in Bezug auf die Gesundheit des Betreuten. Gem. \1901a Abs. 2 kann der Verfasser der Betreuungsverfügung Wünsche für medizinische Maßnahmen festhalten, die für die Betreuerentscheidung gem. \1901 Abs. 3 maßgeblich sind. Die

715 Anders: Hartmann, \1906 schützte allein den Betreuten (Hartmann NStZ 2000, 113, 118); Bienwald/Sonnenfeld/Hoffmann \1906 Rn. 110; einige sehen \1906 der Anwendung eröffnet, wenn es sich auch um den Schutz von Drittinteressen handelt (Soergel/Zimmermann \1906 Rn. 25; Zimmermann Rn. 11; MünchKommBGB/Schwab \$ 1906 Rn. 3; Pardey FamRZ 1995, $713,716)$.

716 Wie z.B. einer alternativen ebenerdigen Schlafform, bei der der Betreute nicht aus dem Bett fallen kann.

717 S. o. \2 C. II. 3.; Freiheit zur Krankheit: BVerfG NJW 1998, 1774 f. BVerfGE 58, 208, 224 ff.; kritisch Coeppicus BtPrax 1999, $130 \mathrm{ff}$.

718 Zimmermann plädiert dafür, das „Wohl des Betreuten“ an dieser Stelle nicht einseitig/ egoistisch zu deuten, sondern vielmehr in Form einer Gesamtbetrachtung (Soergel/Zimmermann \1906 Rn. 25). 
neue Regelung zur Patientenverfügung in \1901a bestimmt nun ausdrücklich die Verbindlichkeit dieser Betroffenenwünsche.

Bei Eingriffen, bei denen die begründete Gefahr des Versterbens oder eines schweren und länger dauernden gesundheitlichen Schadens besteht, hat der Betreuer gem. \1904 Abs. 1 für seine Einwilligung die Genehmigung des Betreuungsgerichts einzuholen. Ohne diese Genehmigung darf die Maßnahme nur durchgeführt werden, wenn mit dem Aufschub Gefahr verbunden ist. Die Nichteinwilligung oder der Widerruf der Einwilligung des Betreuers in eine solche Maßnahme bedarf gem. \ 1904 Abs. 2 ebenfalls der Genehmigung, wenn die Maßnahme medizinisch angezeigt ist und die begründete Gefahr besteht, dass der Betreute aufgrund des Unterlassens der Maßnahme stirbt oder einen schweren und länger dauernden Schaden erleidet. Nach \ 1904 Abs. 3 ist nun festgelegt, dass die Genehmigung zu erteilen ist, wenn die Entscheidung des Betreuers dem Willen des Betreuten entspricht. Sie ist nach \1904 Abs. 4 letztlich nur dann erforderlich, wenn zwischen Betreuer und behandelndem Arzt kein Einvernehmen darüber besteht, dass die Entscheidung des Betreuers dem nach \1901a i.V.m. \1901 Abs. 3 ermitteltem Willen des Betreuten entspricht. Dann kann der Betroffene auch durch einen entsprechenden Wunsch in der Betreuungsverfügung nicht verhindern, dass die Genehmigung des Betreuungsgerichts einzuholen ist, da \1904 insoweit zwingend ist. Weil \ 1904 Abs. 3 dem Betreuungsgericht jedoch vorschreibt, dass dieses die Genehmigung zu erteilen hat, wenn die Entscheidung dem Willen des Betreuten entspricht, und das Betreuungsgericht diesen ebenso wie der Betreuer gem. \ 1901 Abs. 3 entsprechend seiner Wünsche auszulegen hat, kann der Betreuer mittels seiner Wünsche bestimmen, wann die Genehmigung zu erteilen ist.

Das Gesetz schränkt den Verfasser der Betreuungsverfügung daher nur durch das unumgängliche Genehmigungserfordernis im Streitfall zwischen Betreuer und Arzt über den Betreutenwillen ein ( 1904 Abs. 4). Im Übrigen kann der Betroffene Fragen, die seine Gesundheit betreffen, für seine mögliche Betreuung gem. \1901a Abs. 1 in der Patientenverfügung und damit gem. \1901a Abs. 2 S. 1, 1. Alt. auch in der Betreuungsverfügung verbindlich regeln. ${ }^{719}$ Der Einzelne kann in seiner Betreuungsverfügung beispielsweise bestimmen, welcher Arzt ihn behandeln oder untersuchen soll ${ }^{720}$ und Anweisungen betreffend der Vornahme bzw. Nichtvornahme bestimmter Heilbehandlungen ${ }^{721}$ und sonstiger ärztlicher Maßnahmen ${ }^{722}$ geben. Er kann festlegen, ob die Pflege (bei Bereitschaft) durch

${ }^{719}$ Taupitz A 121, er favorisiert für medizinische Angelegenheiten jedoch die mit der Betreuungsverfügung kombinierte oder integrierte Patientenverfügung.

${ }^{720}$ Epple BtPrax 156, 158.

721 Z.B. der Wahl einer Therapieform.

722 Mayer S. 91 - der der Betreuungsverfügung ebenfalls Bindungswirkung zuschreibt; Epple BWNot 1992, 27, 29; Friedrichs MDR 1992, 5, 6 - Friedrichs will die Wünsche der Betreuungsverfügung jedoch nur ,berïcksichtigen“, eine detaillierte Auseinandersetzung mit der Frage der Bindungswirkung der in der Betreuungsverfügung enthaltenen Wünsche nimmt er nicht vor, sagt 
nahe stehende Personen oder durch Fremde (Pflegedienst ${ }^{723}$ ) erfolgen soll, ${ }^{724}$ bzw. von welcher konkreten Person er gepflegt werden will ${ }^{725}$.

b) Fragen des Aufenthalts und des Wohnraums

$\mathrm{Zu}$ untersuchen ist, ob der Einzelne in der Betreuungsverfügung Anweisungen über seinen Aufenthalt während der Betreuung machen kann. Kann er verbindlich festlegen, bis zu seinem Lebensende in seiner Wohnung/ seinem Haus zu bleiben, ein spezielles Pflegeheim auswählen oder den Aufenthalt in einem Krankenhaus verhindern? Ist es trotz $\iint 1907,1908$ i i.V.m. SS 1821, 1822 für den Betroffenen möglich, durch antizipierten Wunsch festzulegen, sein Haus nicht zu veräußern? ${ }^{726}$

a) Aufenthalt

Unter Aufenthalt ist der Ort zu verstehen, an dem sich der Betroffene tatsächlich vorrangig niedergelassen hat. ${ }^{727}$ Das Betreuungsrechts trifft keine speziellen Aussagen über den allgemeinen Aufenthalt des Betreuten. Auch \1907, der die Aufgabe der Mietwohnung regelt, formuliert keine Anordnungen. Daher gilt auch in dieser Frage allein der Maßstab des \ 1901 Abs. 3. Den Wünschen des Betroffenen über seinen Aufenthalt hat der Betreuer daher soweit zu entsprechen, wie sie dessen Wohl nicht zuwiderlaufen. Diese Wünsche werden durch keine weiteren gesetzlichen Regelungen eingeschränkt.

bb) Veräußerung des Wohnraums

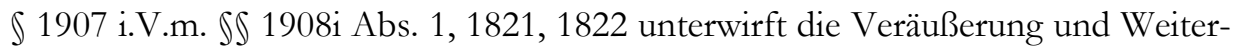
vermietung des im Eigentum des Betreuten stehenden Wohnraums, sowie die Kündigung von gemietetem Wohnraum dem zwingenden Genehmigungsvorbehalt des Betreuungsgerichts. ${ }^{728}$ Es ist zu untersuchen, inwieweit \ 1907 für die Betroffenenwünsche über $\int 1901$ Abs. 3 hinausgehende Anforderungen formuliert.

Der Gesetzgeber wollte mit \1907 den Wohnraum des Betreuten als räumlichen Mittelpunkt des Lebens für den Betreuten vor voreiliger Veräußerung oder Kündigung durch den Betreuer schützen. ${ }^{729}$ Die Heimverschaffung in Verbindung mit der Wohnungsauflösung stellt einen erheblichen Eingriff in die Lebensum-

jedoch zum „Wunschrecht“ bzw. zu der vom Betreuungsrecht geschaffenen „Möglichkeit, in seinen

Angelegenheiten Wünsche gegenüber dem Betreuer vorzubringen: [....] Ein Mitspracherecht ist dies jedoch

nicbt.“(S. 6); Palandt/Diederichsen Einf. v. \1896 Rn. 9; BT-Drucks. 11/4528 S. 142; HK-

BUR/Bauer $\$ 1901$ Rn. 35.

723 Zimmermann Rn. 368.

724 Winkler S. 60, 67.

${ }^{725}$ Cypionka NJW 1992, 207, 210.

${ }^{726}$ Dies bejaht BayObLG NJW-RR 1998, 158 f. = FamRZ 1998, 455.

727 BayObLGZ 1985, 158, 160.

${ }_{728}$ Damrau/Zimmermann S 1907 Rn. 1; Miet- und Eigentumswohnung, BT-Drucks. 11/4528 S. 149.

${ }^{729}$ Damrau/Zimmermann \1907 Rn. 1. 
stände des Betroffenen dar, ${ }^{730}$ sie beeinträchtigt die Rechte eines Menschen in der Unverletzlichkeit der Wohnung nach Art. 13 GG und dem Eigentum an der Wohnungseinrichtung nach Art. 14 GG. ${ }^{731}$ \1907 knüpft daher an das reine Mietverhältnis. ${ }^{732}$ Der Wohnraum sei als vertraute Umgebung des Betreuten und Ort des Bekanntenkreises besonders schützenswert. Diese Bedeutung behalte er auch während eines Krankenhaus- bzw. Heimaufenthalt, stelle dies doch eine Möglichkeit für den Betreuten dar, nach seinem Aufenthalt in seine vertraute Umgebung zurückzukehren. ${ }^{733}$ Die Rechtsprechung kam zu einer vergleichsweise einheitlichen Anwendung des $\int 1907$ im Interesse des Betreuten. ${ }^{734}$ \1907 legt nach der gesetzlichen Begründung und der herrschenden Meinung in Rechtsprechung und Literatur lediglich fest, ob eine Genehmigung des Betreuungsgerichts im Fall der Kündigung oder Aufhebung eines Mitverhältnisses oder der Veräußerung der Eigentumswohnung des Betreuten durch den Betreuer erforderlich ist. ${ }^{735}$ \1907 formuliert somit keine inhaltlichen Voraussetzungen. Vielmehr verweist er auf den allgemeinen Maßstab des $\int 1901$ : Wohl und Wünsche des Betreuten. ${ }^{736}$ Eine objektiv unvernünftige Entscheidung zur Wohnsituation des Betreuten läuft seinem Wohl nicht zuwider, wenn sie seinen Wünschen und Vorstellungen entspricht. ${ }^{737}$

730 Winkler S. 60, 66; v.a. auch damit verbundene Wegfall der Möglichkeit einer Rückkehr.

731 Coeppicus Rpfleger 1996, 425, 432.

732 \ 1907 fordert nicht ein tatsächliches Bewohnen (Damrau/Zimmermann \1907 Rn. 6.) Es fordert kein Genehmigungserfordernis, wenn die Wohnung von vornherein nicht zu Wohnzwecken des Betreuten diente. Die fehlende Genehmigung führt gem. SS 1908i, 1831 zur Nichtigkeit der Kündigung (Schumacher WuM 2003, 190, 194).

733 BT-Drucks. 11/4528 S. 149.

734 Vgl. nur BGH NJW 1986, 2829, 2830; BayObLG NJW-RR 1998, 158 f. und die Ausführungen von Harms Rpfleger 2002, 59 ff. und Bobenhausen Rpfleger 1994, 13 ff.

735 Darüber hinaus wird nach allgemeiner Meinung aus \ 1907 abgeleitet, dass ein Verlassen der Wohnung des Betreuten gegen dessen Willen nur dann die zwangsweise Heimverschaffung ermöglicht, wenn die Voraussetzungen für eine geschlossene Unterbringung gegeben sind (Harm Rpfleger 2002, 59, 61). Die Genehmigung zur Kündigung der Wohnung durch das Betreuungsgericht berechtigt den Betreuer nicht, den Betreuten zwangsweise aus der Wohnung zu verweisen, außer wenn das Gericht die Unerbringung genehmigt hat (Damrau/Zimmermann \1907 Rn. 19). Das Gericht kann nicht aufgrund von $\$ 33$ FGG Zwang anordnen.

736 BT-Drucks. 11/4528 S. 150; BayObLG FamRZ 1998, 455, 456.

${ }_{737}$ Das Recht auf Selbstbestimmung ist auch in objektiv unvernünftigen Entscheidungen zu achten (Harm Rpfleger 2002, 59, 60). Die Diskussion um die Genehmigungsbedürftigkeit der Geschäfte des geschäftsfähigen Betreuten unterstützt diese Aussage. Dabei ist umstritten, ob die vormundschaftliche Genehmigung bei der Kündigung und Vertragsaufhebung des Wohnraums auch dann erforderlich ist, wenn der geschäftsfähige Betreute selbst die Kündigung vornimmt und der Betreuer auf Grund eines Einwilligungsvorbehalts oder „,vorsorglich“" zustimmt. Nach verbreiteter Ansicht kommt in diesem Fall \ 1907 aufgrund des Erforderlichkeitsgrundsatzes nicht zur Anwendung. Der Geschäftsfähige könne selber für sich handeln (HK-BUR/Rink \ 1907 Rn. 6; Schumacher WuM 2003, 190, 194; Erman/Holzhauer $\$ 1907$ Rn. 3 in der Nachfolge: Erman/Roth \$ 1907 Rn. 3). Die gegenteilige Meinung möchte gerade aufgrund des Schutzzwecks der Norm auch das Handeln des Geschäftsfähigen unter $\$ 1907$ subsumieren (Damrau/Zimmermann \$1907 Rn. 3). 
Der Betroffene kann somit jegliche Einzelfragen in Bezug auf seinen Aufenthalt erläutern: Er kann bestimmen, bis wann ein Verbleiben in der eigenen Wohnung erfolgen soll und dass hierbei alle Möglichkeiten der häuslichen Pflege, ggf. eines Wohnungsumbaus und Einschaltung des ambulanten Dienstes auszunutzen sind. ${ }^{738}$ Der Betroffene kann den Einzug in ein Pflegeheim in Bezug auf die Lage der Einrichtung, ihre Trägerschaft, den Zuschnitt und ihre Ausstattung festlegen; er kann die Mitnahme von Wohnungsinventar festlegen, bestimmen, was mit der verbleibenden Wohnungseinrichtung geschieht, ${ }^{739}$ also den Verbleib von Bildern, Schmuck, Erinnerungsstücken, Briefen sowie Sammlungen. ${ }^{740} \mathrm{Ihm}$ steht die Möglichkeit zu, den Ort seines Aufenthaltes oder seiner Unterbringung konkret mit dem Namen des Heims zu bestimmen. ${ }^{741}$ Er kann zusätzliche Leistungen im Krankenhaus oder im Heim wünschen. ${ }^{742}$ Er kann bestimmen, dass keine VeräuBerung erfolgen darf, bzw. nur an Person X, oder auf gar keinen Fall an Person Y und in jedem Fall durch Person $Z$ zu erfolgen hat. Seine Wünsche und Vorstellungen in Bezug auf seinen Aufenthalt im weiten Sinn unterliegen somit einzig der Regelung des \1901. \1907 grenzt die Möglichkeit nicht ein, sondern bestärkt sie. Die Grenze ist hier v.a. in tatsächlichen Fragen, wie der finanziellen Umsetzbarkeit der Wünsche zu sehen. ${ }^{743}$ Lebt der Betreute im Pflegeheim, ist eine im Verhältnis zu seinen Mitteln unverhältnismäßige Wohnung nicht zu erhalten.

c) Vermögenssorge ${ }^{744}$

Unter Vermögenssorge versteht das Betreuungsrecht alle Maßnahmen, die darauf gerichtet sind, das Vermögen des Betreuten zu erhalten, zu verwerten und zu vermehren. ${ }^{745}$ Vermögenssorge ist dabei als fremdnützige rechenschaftspflichtigte Vermögensverwaltung ${ }^{746}$ zu verstehen..$^{77}$

Fraglich ist, ob die betreuungsrechtlichen Regelungen zur Vermögenssorge der

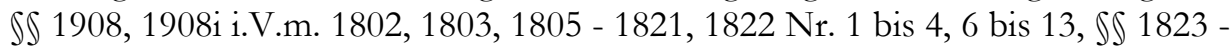
1826, 1828 - 1831 dem Betroffenen mittelbar den Einfluss auf seine Vermögens

\footnotetext{
738 Coeppicus Sachfragen S. 55 - nennt dies dann aber Pflegeverfügung.

${ }^{739}$ Einlagern oder aufteilen, etc: Winkler S. 60, 67.

${ }^{740}$ Coeppicus Sachfragen S. 63; HK-BUR/Bauer \$1901 Rn. 35.

741 Zimmermann Rn. 368; Cypionka NJW 1992, 207, 210; Palandt/Diedericbsen \1901 Rn. 3; OLG Köln NJW-RR 1997, 451.

742 Palandt/Diederichsen \1901 Rn. 3.

743 BayObLG FamRZ 1998, 455, 456.

${ }^{744}$ Fragen der Vergütung werden hier außen vor gelassen und sind in $\ 5$ B. zu diskutieren.

745 Müller/Renner Rn. 125.

${ }^{746}$ Jürgens/Kröger/Marschner/Winterstein Rn. 233.

747 Die Geltendmachung von Unterhaltsansprüchen des Betreuten sowie die Beantragung von Sozialhilfe zählen nach einer Meinung wegen des Unterhalts ersetzenden Charakters nicht dazu (Müller/Renner Rn. 125; LG Köln FamRZ 1998, 919). Nach anderer Meinung ist auch dies von der Vermögenssorge umfasst (LG Regensburg FamRZ 1993, 477 f.; Dodegge NJW 1993, 2353, 2357).
} 
verwaltung durch eigene Wünsche und Vorstellungen in der Betreuungsverfügung verwehren oder ob der Betreute gem. \1901 Abs. 3 nur den Wünschen, Vorstellungen und dem Wohl des Betreuten verpflichtet ist. Kann der Betroffene Wünsche über Geschenke, Ausstattungen oder ähnliche Zuwendungen an Familienangehörige und andere Personen in der Betreuungsverfügung für den Betreuer verbindlich festhalten oder etwa eine Geschäftsübergabe ohne Einschränkung im Rahmen der Betreuungsverfügung regeln?

Im Unterschied zum Minderjährigenrecht ist im Betreuungsrecht nicht davon auszugehen, dass Ziel der Vermögenssorge in jedem Fall die Mehrung des Vermögens des Betreuten ist. ${ }^{78}$ Vielmehr soll der vor der Anordnung der Betreuung erreichte Lebensstandard durch die Verwaltung des Betreuten erhalten bleiben. ${ }^{749}$ Die Vermögensmehrung kommt nur in Betracht, wenn Vermögensbestandteile für den Lebensunterhalt - einschließlich eines ggf. vertretbaren Luxus - nicht benötigt werden. 750

\section{aa) Ausstattung gem. $\$ 1908$}

$\int 1908$ bestimmt, dass der Betreuer eine Ausstattung aus dem Vermögen des Betreuten nur mit Genehmigung des Betreuungsgerichts versprechen oder gewähren kann. Normzweck ist der Schutz des Betreuten und seines Vermögens vor der Vergabe von Ausstattungen, die über das den Verhältnissen des Betreuten entsprechende $\mathrm{Ma}$ hinausgehen, sowie eine entsprechende Kontrolle des Betreuers. ${ }^{751}$ Ausstattung ist nach der Legaldefinition des $\ 1624$, "was [...] auf die Erlangung einer selbständigen Lebensstellung zur Begründung oder zur Erhaltung der Wirtschaft oder der Lebensstellung [...] zugewendet wird" "752.

\1908 könnte bei Beachtung des durch das Betreuungsgericht anzulegenden Maßstabs zu einer Einschränkung der Verfügbarkeit über das eigene Vermögen des Betreuten führen. Soll sie zu dem Betreutenwohl erfolgen, bildet die Vermögensgröße des Betreuten die faktische Grenze der Realisierung. Demnach beschränkt sich die vormundschaftliche Prüfung auf die Frage der faktischen Umsetzbarkeit in Bezug auf das tatsächlich vorhandene Vermögen bei der Realisierung der Betroffenenwünsche nach \1901 Abs. 2 und 3. Die vormundschaftliche Prüfung des $\int 1908$ schränkt den Betreuten in der freien Verfügbarkeit seines Vermögens nur dahingehend ein, dass die Verfügbarkeit sich in diesem Rahmen bewegen muss. Diese Einschränkung erfolgt im Interesse des Betreuten. Geneh-

\footnotetext{
748 Jürgens/Kröger/Marschner/Winterstein Rn. 233; Wesche BtPrax 2003, 56, 60; so jedoch das Ziel des Vormundschaftsrechts: bei der Vermögenssorge der Minderjährigen steht der Erhalt und die Vermehrung ihres Vermögens im Vordergrund, vgl. Bienwald/Sonnenfeld/Hoffmann Anhang zu \1908i Rn. 6.

${ }^{749}$ Schmidt/Böcker/Schmidt Rn. 351; Bienwald/Sonnenfeld/Hoffmann Anhang \1908i Rn. 6; BayObLG FamRZ 1991, 481, 482.

750 Jürgens/Kröger/Marschner/Winterstein Rn. 233.

751 Staudinger/Bienwald \ 1908 Rn. 2; MünchKommBGB/Schwab \1908 Rn. 1.

752 Staudinger/Bienwald \$1908 Rn. 3.
} 
migungspflichtig ist nach \1908 das Versprechen (als Verpflichtungsgeschäft) oder das Gewähren (als Verfügungsgeschäft). Das Gericht kann in dem Genehmigungsverfahren prüfen, ob die Ausstattung das den Vermögensverhältnissen des Betreuten entsprechende $\mathrm{Maß}$ übersteigt. ${ }^{753}$ Maßgebend ist dabei das Wohl des Betreuten. Seinem Wunsch ist nach Maßgabe von \ 1901 Abs. 3 zu entsprechen. ${ }^{754}$ \1908 formuliert keine speziellen inhaltlichen Voraussetzungen und schränkt die antizipierte Entscheidung des Betreuten nur in Hinblick auf das zwingende Genehmigungserfordernis ein.

bb) Schenkungen und andere Vermögensfragen

Das Betreuungsrecht enthält neben \ 1908 keine weiteren ausdrücklichen Regelungen zur Vermögenssorge des Betreuten. \1908i verweist auf Schenkung und Vermögensfragen behandelnde Paragraphen des Vormundschaftsrechts. Dabei weist \ 1908 i nicht alle Verweise auf. 755 In einigen Fällen ist die analoge Anwendung weiterer Vorschriften sinnvoll und sogar zwingend. ${ }^{756}$ Die Regelungen zur Vermögenssorge wirken im Vormundschafts- wie im Betreuungsrechts zum Schutz des Mündels bzw. Betreuten. Auch im Rahmen der Vermögenssorge ist die oberste Pflicht des Betreuers die Erfüllung der Wünsche des Betreuten und die Beachtung seines Wohls nach \ 1901 Abs. 2 und 3.757 Die Verweise in die Regelungen des Vormundschaftsrechts geben für die Schenkung des Betreuten andere inhaltlichen Vorgaben und führen daher zu einer Beschränkung der Betreutenwünsche. ${ }^{758}$ Nach SS 1908 i Abs. 2 i.V.m. 1804 kann der Betreuer Schenkungen in Vertretung des Betreuten nur vornehmen, die aufgrund einer sittlichen Pflicht oder einer auf den Anstand zu nehmenden Rücksicht erfolgen oder dem Wunsch des Betreuten entsprechen und nach seinen Lebensverhältnissen üblich sind. ${ }^{759}$

Den \$S 1804, 1908 i Abs. 2 S. 1 liegt der Gedanke zugrunde, Schenkungen aus dem Vermögen des Betroffenen entsprächen nicht seinem wohlverstandenen Interesse; der Betreuer als Verwalter eines fremden Vermögens solle es erhalten, nicht vermindern. ${ }^{760}$ Was Pflicht- und Anstandsgeschenke nach $\int 1804$ sind, wird

\footnotetext{
753 Staudinger/Bienwald \$ 1908 Rn. 7.

754 MünchKommBGB/Schwab S 1908 Rn 7; Staudinger/Bienwald S 1908 Rn. 7.

755 Einige Verweise wurden im Gesetzgebungsverfahren durch nachträgliches Einfügen mancher

Regelungen vergessen, siehe dazu: Soergel/Zimmermann \1908i Rn. 2; Palandt/Diederichsen $\int 1908$ i Rn. 1.

756 Soergel/Zimmermann $\int 1908$ i Rn. 2.

757 Jürgens/Kröger/Marschner/Winterstein Rn. 233; Wesche BtPrax 2003, 56, 60; Schmidt/Böcker/Schmidt Rn. 351.

758 Separat zu diskutieren ist dies bei Fragen der Vergütung des Betreuers, vgl. dazu: $\int 5$ B.

${ }^{759}$ Die Regelungen über Zuwendungen und Schenkungen beschränken diese Freiheit des Betreuten.

Das wird nicht genügend berücksichtigt von Epple BtPrax 1993, 156, 158; Dodegge/Roth C. Rn. 163 .

760 Motive zum BGB Band IV, S. 1106; Staudinger/Engler \1804 Rn. 1; Gernhuber/Coester-Waltjen $\int 61$ Rn. 11.
} 
objektiv bestimmt. ${ }^{761} \int 1908$ i Abs. 2 erweitert die Möglichkeit zu Schenkungen auf übliche Gelegenheitsgeschenke. ${ }^{762}$ Was unter diesen zu verstehen ist, kann objektiv bestimmt werden. Nach $\int 1908$ i Abs. 2 S. 1 a.E. sind sie vorzunehmen, wenn dies dem Wunsch des Betreuten entspricht und nach dessen Lebensverhältnissen üblich ist. Das strenge Schenkungsverbot soll durch \1908i Abs. 2 jedoch nicht aufgeweicht, sondern nur ,vorsichtig“ erweitert werden, so die gesetzliche Begründung. ${ }^{763}$

Die Pflicht-, Anstands- oder Gelegenheitsgeschenke kann der Betroffene umfassend in der Betreuungsverfügung bestimmen. ${ }^{764}$ Alle anderen Schenkungen sind dem Betreuer verboten. Dies gilt auch dann, wenn sie der Betreute ausdrücklich wünscht. Da der Betreuer eine Gelegenheitsschenkung nach dem Gesetzestext nur auf Wunsch des Betreuten hin vornehmen darf, existiert ein subjektives Element, ${ }^{765}$ was der grundsätzlichen Ausrichtung des Betreuungsrechts auf die Wünsche und Vorstellungen des Betreuten entspricht. Das generelle Schenkungsverbot der $\iint 1908 i, 1804$ schränkt den Betreuten, in seinem Recht aus Art. 14 Abs. 1, Art. 2 Abs. 1 GG, frei und selbstbestimmt über sein Vermögen verfügen zu können, unverhältnismäßig ein. ${ }^{766}$ „Das unbedingte Schenkungsverbot nimmt dem Betreuten die "Schenkungsfähigkeit", obne dass dies zu seinem Schutz erforderlich wäre. "767 Dem Missbrauch ${ }^{768}$ im Umgang mit dem Betreutenvermögen durch den Betreuer könnte man durch ein vormundschaftliches Genehmigungserfordernis vorbeugen. ${ }^{769}$ Dies wäre mit Blick auf die Rechtsverletzung aus Art. 14 Abs. 1, Art. 2 Abs. 1 GG die weniger eingreifende Rechtsmaßnahme. ${ }^{770}$ Geboten ist deshalb eine teleologische Reduktion des Schenkungsverbots auf eine Genehmigungspflicht bei den Schenkungen, die nicht Pflicht-, Anstands- oder übliche Gelegen-

\footnotetext{
761 Vgl. BayObLG BtPrax 1998, 72, 74; BayObLG FamRZ 1996, 1359, 1360; Böhmer MittBayNot 1996, 405, 406 ff.; Holzhauer FamRZ 2000, 1063, 1064.

762 BT-Drucks. 11/4528 S. 160.

763 BT-Drucks. 11/4528 S. 160; In jedem Fall sind die allgemeinen rechtlichen Grenzen zu beachten: Schenkungen zu Lasten des Trägers der Sozialhilfe oder zur Erlangung sonstiger subsidiärer Sozialleistungen sind stets unwirksam (Dodegge/Roth C. Rn. 163).

764 OLG Karlsruhe NJW-RR 2000, 1313, 1314; Dodegge/Roth C. Rn. 163; Lipp Vorsorgeverfügungen \18 Rn. 93 ff.; Grziwotz.ZEV 2005, 338, 340; Soergel/Zimmermann \1908i Rn. 18; Staudinger/Engler \1804 Rn. 19; dagegen: Palandt/Diederichsen \$1908i Rn. 17; MünchKommBGB/Schwab \$ 1908i Rn. 39.

765 Ausführlich dazu Kollmer S. 224 ff.

766 So auch: Lipp Vorsorgeverfügungen \18 Rn. 98.

${ }^{767}$ Lipp Vorsorgeverfügungen \18 Rn. 98.

768 Missbrauch wird als Grund für das umfassende Schenkungsverbot durch den Betreuer gesehen (BayObLG FamRZ 1996, 1359, 1360). Zustimmend: Böhmer MittBayNot 1996, 405, 410 dort Fn. 71; ähnlich: MünchKommBGB/Schwab \1908i Rn. 39 ff.

769 Bobenhausen BtPrax 1994, 158, 160 f.; Böhmer MittBayNot 1996, 405, 410; Canaris JZ 1987, 993 , 999; insofern zustimmend auch MünchKommBGB/Schwab \$ 1908i Rn. 39.

770 Derzeitige Regelung als unverhältnismäßiger Rechtseingriff: Lipp Vorsorgeverfügungen $\ 18$ Rn. 98; Canaris JZ 1987, 993, 998 f.; Holz̧ hauer FamRZ 2000, 1063, 1068; Griviwotz ZEV 2005, 338, $339 \mathrm{f}$.
} 
heitsgeschenke sind, deren Vornahme aber vom Betreuten gewünscht wird, da dem Gesetzgeber hier keine Regelungsalternativen zur Verfügung stehen. ${ }^{771}$

Aufgrund der Unsicherheiten einer Schenkung durch den Betreuer, empfiehlt sich, bei der Konzeption der Vorsorgeregelung auf alternative Gestaltungsmöglichkeiten wie der Verfügung von Todes wegen oder einer (verfrühten) Schenkung mit einer (aufschiebenden) Bedingung durch den Betroffenen selbst, zurückzugreifen. Sofern der Betroffene bei der Abfassung der Vorsorgeregelung nicht unmittelbar nach $₫ 104 \mathrm{Nr}$. 2 geschäftsunfähig ist oder einem Einwilligungsvorbehalt unterliegt, kann er in vollem Umfang über sein Vermögen verfügen. Somit kann er Schenkungen aus seinem Vermögen uneingeschränkt vornehmen und sogar seinen künftigen Betreuer bedenken. ${ }^{772}$

\section{cc) Zwischenergebnis}

Mittels der Betreuungsverfügung ist die Vermögensverwaltung durch den Betreuer während der Betreuung nur zum Teil regelbar. ${ }^{773}$ Darunter fallen Fragen zum Vorgehen im Fall, dass das Vermögen nicht zur Deckung der Lebenskosten (Pflege, Wohnen etc.) ausreicht - z.B. welche Teile des Eigentums veräußert werden können ${ }^{774}$ - wie auch die Grundaussage, ob das Vermögen eher zusammengehalten oder möglichst viel für eine gute Versorgung und Pflege ausgegeben werden soll175. Antizipierte Entscheidungen über die Geldanlage gem. SS 1908i, $1806^{776}$ und Gelegenheitsgeschenke nach $\ 1908$ i Abs. 2 S. 1777 sind möglich. Geregelt werden kann so die Höhe des monatlichen Taschengeldes, die Aufrechterhaltung von Mitgliedschaften in Vereinen, die Weiterzahlung von Spenden oder traditioneller Geschenke an Angehörige 778 . Möglich ist, Anreize für Einzelpflichten wie der Entschädigung für Einzelausfahrten oder Spaziergänge ${ }^{779} \mathrm{zu}$ treffen. ${ }^{780}$

Die Regelungen zur Vermögenssorge des Betreuten schützen primär die Verwirklichung seiner Wünsche und Vorstellungen und erlauben so eine antizipierte Festlegung durch den Einzelnen in seiner Betreuungsverfügung. Das absolute Schenkungsverbot nach \$S 1908 i Abs. 2 S. 1, 1804 wird durch die Ausnahmen in S. 2 a.E. gelockert und bedarf einer teleologischen Reduktion, bei Schenkungen, die durch den Betreuten gewollt sind.

\footnotetext{
771 Holzhauer FamRZ 2000, 1063, 1068; Canaris JZ 1987, 993, 999; Lipp S. 163 f.; Erman/Roth \$ 1908i Rn. 37 f.; Soergel/Zimmermann \1908i Rn. 17; anders dagegen die wohl noch h.M., vgl. MünchKommBGB/Schwab \$1908i Rn. 39.

772 MünchKommBGB/Schwab \$1908i Rn. 44.

773 Epple BWNotZ 1992, 27, 29 f.; Dodegge/Roth C. Rn. 163.

774 Winkler S. 60, 67.

775 Winkler S. 60, 67.

776 Zimmermann Rn. 368; Cypionka NJW 1992, 207, 210.

777 Zimmermann Rn. 368.

778 Coeppicus Sachfragen S. 63.

779 Epple BtPrax 1993, 156, 158.

${ }^{780}$ Str.; vgl. dazu auch die Ausführungen unter $\ 5$ B.
} 
d) Sonstige Angelegenheiten

Fraglich ist, welche gesetzlichen Regelungen neben den einschlägigen Bestimmungen zur Gesundheitssorge, zum Aufenthalt und zum Vermögen des Betreuten diesen in der antizipierten Festlegung seiner Wünsche und Vorstellungen einschränken. Hier sind v.a. Regelungen zu beachten, die ein persönlichen Entscheiden oder Handeln des Betroffenen fordern. Wünsche und Vorstellungen des Betroffenen können nur dann von dem Betreuer umgesetzt werden, wenn ihre Umsetzung durch einen gesetzlichen Vertreter rechtlich möglich ist. Grenzen der gesetzlichen Vertretungsmacht des Betreuten bestehen dort, wo die höchstpersönliche Natur des Geschäfts eine Stellvertretung verbietet bzw. das Gesetz ausdrücklich ein persönliches Handeln verlangt. ${ }^{781}$ Der Betreute ist - unabhängig von seinem Krankheitsbild - in dem Kernbereich seines Persönlichkeitsrechts völlig autonom ${ }^{782}$. Dies betrifft unter anderem die Eheschließung nach $₫ 1311$, die Eingehung einer eingetragener Lebenspartnerschaft gem. \1 Abs. 1 LPartG, die Errichtung oder den Widerruf letztwilliger Verfügungen nach $\int S$ 2064, 2274 sowie die Abgabe einer Sorgerechtserklärung nach \$1626c Abs. 1. Des Weiteren kann der Betreuer gem. $\iint 1795$ Abs. 2, 181 den Betreuten nicht wirksam bei Insichgeschäften vertreten. ${ }^{783}$ Dem Betreuer sind zudem gem. SS 1908i Abs. 1 i.V.m. 1795 einzelne Angelegenheiten entzogen, wenn die Interessen des Betreuten mit denen des Betreuers kollidieren. Somit können diesbezügliche Wünsche und Vorstellungen des Betroffenen in der Betreuungsverfügung zwar Niederschlag finden, sie entfalten jedoch keine rechtliche Wirksamkeit.

\section{Zwischenergebnis}

Der Gesetzgeber hat für die Führung der Betreuung die $\int \ 1901$ ff. auf den Betreuten ausgerichtet, indem er dessen Wünsche und Vorstellungen gem. \1901 Abs. 2 und 3 Verbindlichkeit gewährt. Durch antizipierte Äußerungen kann der Betroffene in der Betreuungsverfügung Entscheidungen zu allen Bereichen seines Lebens treffen. ${ }^{784}$ Das Betreuungsrecht schränkt die Möglichkeiten der vorsorglichen Regelung von Fragen der Gesundheit, des Aufenthaltes und des Vermögens zum Teil ein. Lediglich $\$ \$ 1905$ und 1906 formulieren inhaltliche Anforderungen. Alle übrigen gesetzlichen Regelungen verweisen auf den allgemeinen Maßstab und somit auf $\$ 1901$ Abs. 2 und 3. In diesen Fällen ist allein maßgeblich, was der Betroffene wünscht.

Art und Zahl der verbindlichen, im Rahmen der Betreuungsverfügung antizipiert äußerbaren Wünsche ist unbegrenzt. ${ }^{785}$ Mittels der Betreuungsverfügung

\footnotetext{
781 Müller/Renner Rn. 127 ff.

782 Kollmer S. 92.

783 Müller/Renner Rn. 139.

784 Bienwald/Sonnnenfeld/Hoffmann \1901 Rn. 22; Perau MittRhNotK 1996, 285, 286; HKBUR/Bawer $\$ 1901$ Rn. 35.

785 Perau MittRhNotK 1996, 285, 286 und 288.
} 
kann der Betroffene vom Verbleib seiner Haustiere ${ }^{786}$ bis zur Auswahl seines Pflegedienstes alles Erdenkliche für sein Leben und sein Ableben festlegen.

IV. Stellung, Aufsicht und Pflichten des Betreuers

Der Betreuer ist dem Wohl des Betreuten verpflichtet und deshalb grundsätzlich im jeweiligen Einzelfall an den Wunsch des Betreuten gebunden, sofern sich dieser damit nicht aufgrund seiner eingeschränkten Eigenverantwortlichkeit selbst zu schädigen droht (\$ 1901 Abs. 2, 3 S. 1). Die Einhaltung dieser Pflicht hat zunächst das Betreuungsgericht zu überwachen. ${ }^{787}$ Fraglich ist, ob der Betroffene mittels der Betreuungsverfügung eine Haftungserleichterung für den Betreuer bestimmen kann. Gem. \1833 i.V.m. \1908i Abs. 1 S 1 haftet der Betreuer für einen Schaden, der durch unbegründetes Übergehen eines Wunsches des Betreuten durch den Betreuer entsteht ${ }^{788}$. Der Betreuer hat seine Angelegenheiten so zu erledigen, dass es zu keiner schuldhaften Pflichtverletzung gegenüber dem Betreuten kommt ${ }^{789}$. Dies gilt auch für die pflichtmäßig einzuholende vormundschaftliche Genehmigung in den gesetzlich vorgesehenen Fällen. ${ }^{790}$ Sonst haftet er gem. $\int 1833$ i.V.m. 1908 i Abs. 1 auf Schadensersatz ${ }^{791}$ und ist ggf. strafrechtlichen Folgen ausgesetzt. ${ }^{792} \mathrm{Da} \int 1833$ nicht dispositiv ist, ist eine individuelle Regelung nicht möglich. ${ }^{793}$ Sollte sie getroffen werden, ist sie für den Betreuer nicht verbindlich. So dass der Betreuer nicht von der Ersatzpflicht eines auf einer Pflichtverletzung beruhenden Schadens gem. \1908i Abs. 1, 1833 durch den Betreuten freigesprochen werden kann. ${ }^{794}$ Zum Teil wird hier eine erbrechtliche Lösung vorgeschlagen, die eine verbindliche Regelung ermöglicht, nach der die Erben des Betreuten gegen den Betreuer keine Schadensersatzansprüche geltend machen dürfen: eine Auflage gem. \2192 oder ein Vermächtnis nach \ 2174 an den Betreuer. Dies müsste wegen des erbrechtlichen Charakters aber in Testamentsform erfolgen. ${ }^{795}$

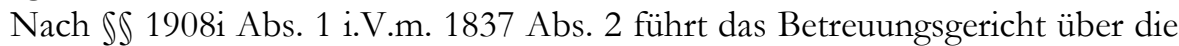
Tätigkeit des Betreuers Aufsicht. Dazu muss der Betreuer diesem gem. \S $1898 \mathrm{i}$

\footnotetext{
786 Coeppicus Sachfragen S. 63.

${ }^{787}$ Staudinger/Bienwald \ 1901 Rn. 11 f.; Erman/Holz̧hauer \$ 1901 Rn. 7.

788 MünchKommBGB/Schwab \1901 Rn. 19; Zimmermann Rn. 369 und FamRZ 1991, 270 ff.

${ }^{789}$ BT-Drucks. 11/4528 S. 141.

790 Sowohl im Rahmen der allgemeinen Aufsicht nach \§ 1908i Abs.1 S. 1, 1837 Abs. 2, 3, 1839 1841, 1843 als auch bei der betreuungsgerichtlichen Genehmigung nach \$\$ 1904 - 1907, $1908 \mathrm{i}$ Abs. 1 S. 1, 1810 S. 2, 1811, 1812 Abs. 3, 1814 - 1816, 1819 - 1821, 1822 Nr. 1 - 4, 6 - 13, 1823,1825 .

${ }^{791}$ Dies gilt auch bei Vereins- oder Behördenbetreuern; werden Mitarbeiter zum Einzelbetreuer bestellt, haften sie als natürliche Person.

792 Vgl. z.B. \266 Strafgesetzbuch.

793 Zimmermann Rn. 369; Epple BWNotZ 1992, 27, 29.

${ }^{794}$ Somit kann er zumindest von den Erben des Betreuten und den Nachfolgebetreuern in Anspruch genommen werden, wenn ihm bei der Wahrnehmung der Betreuung ein Fehler unterlaufen ist.

795 Zimmermann Rn. 369.
} 
Abs. 1 i.V.m. 1839 und 1840 Auskunft erteilen und nach \$S 1908i Abs. 1 i.V.m. 1802 ein Vermögensverzeichnis des Betreuten erstellen. Bei diesen Normen handelt es sich um Ge- und Verbote zur pflichtgemäßen Fürsorge durch das Betreuungsgericht. ${ }^{796}$ Sie sind Grundlage der gerichtlichen Kontrolle. Als solche sind sie nicht durch den Betroffenen mittels abweichender Wünsche der Betreuungsverfügung abdingbar. ${ }^{797}$ Gleiches gilt für die Mitteilungspflicht des Betreuers, wenn Umstände bekannt werden, die eine Betreuungsänderungsanordnung notwendig werden lassen, vgl. \1901 Abs. 5.798

Endet die Betreuung durch Aufhebung, Entlassung des Betreuers oder Tod des Betreuten, ist der Betreuer unter Mitwirkung des Gegenbetreuers (Gegenvormundes) verpflichtet, über die Verwaltung Rechnung zu legen und das verwaltete Vermögen herauszugeben, vgl. \1908i i.V.m. \\ 1890 - 1892. Stirbt der Betreuer, so muss nach den Grundsätzen für die erste Bestellung gem. \$1908c ein neuer bestellt werden. ${ }^{799}$ Auch bei diesen Regelungen handelt es sich um zwingendes Recht zum Schutz des Betreuten, welches nicht durch seine antizipierten Wünsche der Betreuungsverfügung abgedungen werden können.

\section{B. Vergütung des Betreuers}

Grundsätzlich wird die rechtliche Betreuung unentgeltlich ehrenamtlich geführt, vgl. \S 1908 i Abs. 1, 1836 Abs. 1 S. 1. In diesem Fall erfolgt lediglich ein Aufwendungsersatz des Betreuers. Ausnahmsweise kann die Betreuung entgeltlich geführt werden, wenn sie entweder berufsmäßig, vgl. $\int S 1908$ i Abs. 1, 1836 Abs. 1 S. 2 oder zwar ehrenamtlich geführt wird, der Umfang oder die Schwierigkeit der vormundschaftlichen Geschäfte der Betreuung jedoch eine angemessene Vergütung rechtfertigen, vgl. \S1908i Abs. 1, 1836 Abs. 2. In diesen Fällen erhält der Betreuer eine Vergütung und einen Aufwendungsersatz.

\section{Mittellosigkeit des Betreuten}

Die Vergütung erfolgt bei mittellosen Betreuten aus der Staatskasse, vgl. $\int \$$ 1908i, 1835 Abs. 4, 1835a Abs. 3, 1836 d. Der Staat kann den Betreuten oder seine Erben in Regress nehmen, $\iint 1908$ i Abs. 1, 1836e. Die Vergütung wird durch

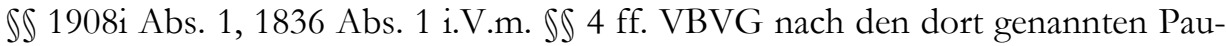

\footnotetext{
796 MünchKommBGB/Wagenitz \1839 Rn. 1, \1840 Rn. 3.

${ }^{797}$ Staudinger/Bienwald \1908i Rn. 110.

798 BT-Drucks. 11/4528 S. 135; Weitere Mitteilungspflichten ergeben sich aus $₫ 6$ Abs. 3 S. 2 Bundesseuchengesetz (der Meldepflicht für den Betreuer, welchem die Personensorge für einen Betroffenen zusteht) und aus $₫ 43$ Abs. 1 Waffengesetz (die Anzeigepflicht des Betreuers im Fall des Erwerbs einer erlaubungspflichtigen Schusswaffe durch den Betreuten) vgl. MünchKommBGB/Schwab \$ 1901 Rn. 28.

799 Jürgens/Kröger/Marschner/Winterstein Rn. 150.
} 
schalen festgelegt. ${ }^{800}$ Diese Festsetzung erfolgt durch das Betreuungsgericht, dem diesbezüglich kein Ermessen zusteht. 801 Weil der Betreuer in diesem Fall aus der Staatskasse vergütet wird, kann die Vergütung des Betreuers eines mittellosen Betreuten nicht individuell durch die Wünsche des Betroffenen mittels einer Betreuungsverfügung ausgestaltet werden. ${ }^{802}$ Sie unterliegt den gesetzlichen Regelungen der \$S 1908 i Abs. 1, 1836 i.V.m. IS 4 ff. VBVG.

\section{Vermögende Betreute}

Bei vermögenden Betreuten wird die Vergütung und der Aufwendungsersatz aus deren Privatvermögen beglichen, vgl. SS 1908 i Abs. 1, 1836 c. Das Gesetz sieht eine ausdrückliche Einflussnahme durch den Betreuten nicht vor. Die Regelungen der Betreuervergütung sind zum Großteil zwingend. ${ }^{803}$ Die Stundensätze sind nicht durch einen monatlichen Pauschalbetrag oder einen Prozentsatz des verwalteten Vermögens abdingbar. ${ }^{804}$ Eine individuelle Einflussnahme ist auf die Bereiche beschränkt, in denen das Gesetz nicht zwingend ist, für das Betreuungsgericht also ein Ermessen in der Festsetzung besteht. Fraglich ist, inwieweit der Betroffene mittels seiner Betreuungsverfügung auf die Vergütung seines Betreuers individuell Einfluss nehmen kann.

\section{Individuelle Vergütung des Berufsbetreners}

a) Vormünder- und Betreuervergütungsgesetz

Der Berufsbetreuer erhält Vergütung und Aufwendungsersatz nach den Pauschalen der $\iint 1908$ i Abs. 1, 1836 I und \ 4 Abs. 2 VBVG. Über die Tätigkeit als Betreuer hinaus kann der Betreuer für besondere berufliche Dienste eine Vergütung als Aufwendungsersatz nach \4 Abs. 2 VBVG und \ 1836 Abs. 3 gesondert geltend machen. Fraglich ist, ob diese Regelungen eine individuelle Festsetzung der Vergütung durch den Betreuten untersagen. Sein Vermögen darf er als sein Eigen-

\footnotetext{
800 Die Pauschalen setzen sich aus sog. Stundensätzen zusammen, die sich nach folgenden Kriterien richten: \4 Abs. 1 VBVG: nach der Ausbildung des Betreuers; $\ 5$ Abs. 1 und 2 VBVG: nach der Dauer der Betreuung.

801 OLG Hamm FamRZ 2003, 116; Lipp FS Bienwald S. 177, 189; Palandt/Diederichsen Anhang zu \1836 Rn. 1; HK-BUR/Bauer/Deinert \1836 Rn. 69; MünchKommBGB/Wagenitz Vor \1836 Rn. 14.

802 Palandt/Diederichsen \1836 Rn. 6, 11.

803 Jürgens/Jürgens \4 VBVG Rn. 2 ff.; MünchKommBGB/Wagenitz』1836 Rn. 5 sieht jedoch die Möglichkeit eines separate Dienst- oder Werkvertrages für Betreuungsaufgaben.

${ }^{804}$ HK-BUR/Bauer/Deinert $\$ 1836$ Rn. 72; BR-Drucks. 960/96 S. 26; so auch schon BayOLG FamRZ 1992, 854,855; OLG Frankfurt/Main FamRZ 1993, 603, 604. Eine Erhöhung der Vergütung erfolgt gesetzlich nur, soweit der Betreuer über seine Tätigkeit als Betreuer hinaus zusätzliche Dienste für den Betreuten erbringt (\$\ 1908 i Abs. 1, 1835 Abs. 3 i.V.m. \ 4 Abs. 2 S. 2 VBVG). Dies ist jedoch nicht als Erhöhung der gesetzlichen Pauschalen zu sehen, sondern als Vergütung einer Leistung im Rahmen der regulären beruflichen Tätigkeit des Betreuers.
} 
tum nach seinen Vorstellungen verwenden, vgl. Art. 14 GG, \ 1901 Abs. 3. $\int 5$ VBVG bestimmt die Vergütung des Betreuers eines vermögenden Betreuten.

Das VBVG ist seit Juli 2005 in Kraft. Die vorher geltende Vergütungsordnung 805 ermöglichte eine individuelle Fallvergütung, die nach Zeitaufwand abzurechnen war. Mit Einführung des VBVG sollten zum einen die Stundensätze vereinheitlicht werden und zum anderen eine Entbürokratisierung für die Betreuer und das Betreuungsgericht erzielt werden. ${ }^{806}$ Nach der alten Regelung des BVormVG ${ }^{807}$ musste jede im Rahmen der Betreuung geleistete Stunde durch den Betreuer für den Rechtspfleger nachvollziehbar aufgelistet und begründet werden. Der dafür erforderliche Zeit- und Kraftaufwand sollte durch die neue Regelung der pauschalen Fallvergütung vermieden werden, der Betreuung zugute kommen und die Missbrauchsgefahr ${ }^{808}$ verringern. Aufgrund des eine weitere Beschwerde ausschließenden früheren Verweises der Vergütungsregelungen auf das ZSEG ${ }^{809}$ entwickelte sich nach dem alten Recht eine Judikatur mit Streitigkeiten über Abrechnung und Bemessung der Vergütung. ${ }^{810}$ Diese belasteten die Betreuungsgerichte durch hohe Fallzahlen unverhältnismäßig und beanspruchten den Justizfiskus durch zum Teil erheblich überzogene Vergütungserwartungen mancher Berufsbetreuer. ${ }^{811}$

Mit Einführung des VBVG sollte eine einheitliche, nicht durch Ausnahmen geprägte Regelung getroffen werden. Die Regelungen des VBVG sind daher abschließend und zwingend und lassen keine Ausnahmen in Form von individuellen Abreden zu. ${ }^{812}$ Diesbezüglich lässt sich weder mittels einer Betreuungsverfügung von Seiten des Betreuten, noch durch das Betreuungsgericht eine andere Pauschale treffen. $\int 5$ VBVG ist in das abschließende gesetzliche System einbezogen. ${ }^{813}$

\footnotetext{
805 BGB i.V.m. BVormVG.

806 BR-Drucks. 865/03 S. 1; OLG Braunschweig BtPrax 2007, 32, 35; Jurgeleit/Jurgeleit \ 4 VBVG

Rn. 5 - 7 und $\$ 5$ VBVG Rn. 4 - 6.

807 Berufsvormündervergütungsgesetzes.

$808 \mathrm{Da}$ der Betreuer nun überhaupt keine Stunden mehr darlegen muss, kann er auch keine darlegen, die er nicht geleistet hat.

809 Zeugen- und Sachverständigenentschädigungsgesetz.

810 MünchKommBGB/Wagenitz Vor \1835 Rn. 4.

811 MünchKommBGB/Wagenitz Vor $\$ 1835$ Rn. 4.

812 Lipp FS Bienwald S. 177, 189.

${ }^{813}$ Das OLG Braunschweig befasste sich mit dem VBVG aufgrund einer Klage eines Betreuers, der sich durch die $\$ \$ 4,5$ VBVG in seinen Grundrechten verletzt sah. Das OLG sieht die pauschalen Stundensatzvergütung nach $\$ ₫ 4$ Abs. 1, 5 VBVG aufgrund fehlender Ausnahmeregelungen für besonders aufwendige und schwere Betreuungen im Fall des bemittelten Betreuten als verfassungswidrig an (OLGR Braunschweig 2007, 94, 94). $\int 4$ Abs. 2 S. 1 VBVG bewertet es als nicht mit dem GG vereinbar, soweit danach der Stundensatz auch Kosten für Aufwendungen des Berufsbetreuers abdeckt, die nicht Aufwendungen im Sinne des $₫ 1835$ Abs. 3 darstellen und nicht zu den gewöhnlichen, mit der Führung von Betreuungen regelmäßig verbundenen, allgemeinen Kosten gehören. Insofern sah das OLG $\iint 4,5$ VBVG als mit Art. 12 Abs. 1, Art. 3 Abs. 1 GG unvereinbar. Das BVerfG wies die Vorlage als unzulässig ab. Es fehlte an einer sorgfältigen Prüfung der Entscheidungserheblich- und der Verfassungsmäßigkeit der als verfas-
} 
Sinn und Zweck, ihre historische Entwicklung und ihr klarer Wortlaut erlauben keine abweichende Bestimmung der Vergütung des Berufsbetreuers. Eine individuelle Ausgestaltung der Vergütung - abweichend von den VBVG durch die Betreuungsverfügung - ist bei Berufbetreuern nicht möglich. Die Regeln des VBVG sind diesbezüglich zwingend und abschließend.

\section{b) Alternative Vereinbarungen}

Zur individuellen Ausgestaltung der Vergütung erscheint eine testamentarische Berücksichtigung möglich, die Vergütung durch separaten und zusätzlichen Dienstleistungsvertrag oder durch Schenkung.

Die Gefahr des Missbrauchs durch den Betreuer lässt sich im Rahmen privatautonomer Regelungen nur bedingt begegnen. Bei der testamentarischen Berücksichtigung kann dem Einwand der Erben mit Verweis auf den Pflichtteilsergänzungsanspruch nach $\ 2325$ begegnet werden. Dieser schützt den Erben im Wege der Vermögensnachfolge in hinreichendem Maße, zumal es um die Frage der Verfügungsbefugnis des Betreuten im Rahmen der Selbstbestimmung über sein Vermögen geht. Eine testamentarische Berücksichtigung des Betreuers ist jedoch mit Blick auf die Ziele des Betreuungsrechts kritisch zu bewerten. Der Betreuer erhält den Vorteil der höheren Vergütung nicht während der Führung der Betreuung, sondern erst nach deren Abschluss mit Eintritt des Todes des Betreuten. Der Betreute wird seinen Betreuer i.d.R. für seinen Zeit- und Leistungsaufwand während der Zeit der Betreuung parallel zu dieser materiell belohnen wollen. Dies entspräche auch den Zielen des Betreuungsrechts, das ausschließlich zu Lebzeiten Anwendung findet. Einer testamentarischen Berücksichtigung des Betreuers stehen die derzeitigen gesetzlichen Regelungen nicht entgegen. Die dem Einzelnen durch das VBVG aufgelegte Beschränkung der freien Ausübung seines Selbstbestimmungsrechts über sein Vermögen kann folglich aufgewogen werden.

Ein neben der Vergütung separater und zusätzlicher Dienstleistungsvertrag oder eine Schenkung würde das System der Vergütung missachten und wäre daher möglicherweise wegen des zwingenden Charakters des VBVG gem. \134 nichtig. Individuell vereinbarte Honorare könnten Erfolgshonorare sein, so dass in dieser Form zum einen eine hohe Missbrauchswahrscheinlichkeit, zum anderen ein Konflikt mit dem Wettbewerbsrecht entstehen könnte. ${ }^{814}$ Die Möglichkeit, eine Vergütung durch individuelle Regelungen zu treffen, bildet daher nach neuem Recht keine Lösung.

sungswidrig erachteten Vorschriften. Das BVerfG merkte in seiner Begründung an, dass sich für die gerichtliche Vergabepraxis bei Betreuungen die Verfassungswidrigkeit der Normen $\$ \int 4,5$ VBVG nicht begründen ließe (BVerfGE 1 BvL 10/06 vom 06.02.2007 Rn. 27). Die Regelung der Berufsbetreuervergütung sei zudem nicht anhand eines Einzelfalles, sondern im Verbund mehrerer Betreuungen und folglich im Rahmen der sog. Mischkalkulation vorzunehmen. Danach soll ein Betreuer gleichermaßen mit aufwendungsintensiveren wie mit weniger aufwendigen Betreuungen betraut werden.

814 Bienwald Rpfleger 2002, $423 \mathrm{ff}$. 


\section{Individuelle Vergütung des ehrenamtlich tätigen Betreners}

a) Gestaltungsmöglichkeiten durch die Betreuungsverfügung

Der ehrenamtlich tätige Betreuer erhält gem. $\ 1835$ grundsätzlich keine Vergütung, ${ }^{815}$ lediglich nach $\int \ 1908$ i Abs. 1, 1835 Abs. 1 - 4 einen Aufwendungsersatz. ${ }^{816}$ Diesen kann er nach $\ 1835$ a statt in einer Einzelabrechnung in Form einer pauschalen Entschädigung verlangen. Ausnahmsweise kann eine angemessene Vergütung bei vermögenden Betreuten nach Ermessen des Gerichts festgesetzt werden, ${ }^{817}$ vgl. $\int S$ 1908i Abs. 1, 1835a Abs. 1 S. 1, 1836 Abs. 2. Nur bei dem vermögenden Betreuten steht dem Gericht ein pflichtgemäßes Ermessen zu, ${ }^{818}$ welches mittels einer Betreuungsverfügung beeinflusst werden könnte.

Die Anerkennung dieses Ermessens ist in der Rechtssprechung bislang wenig gesichert. ${ }^{819} \ 1836$ Abs. 2 begrenzt seinem Wortlaut nach die Bewilligung des Gerichts einer angemessenen Vergütung, soweit der Umfang oder die Schwierigkeit der betreuungsrechtlichen Geschäfte dies rechtfertigen. Der Wortlaut gibt die Bemessung der Vergütung nicht der Gestaltungsmöglichkeit mittels Wünschen nach einer Vergütung des ehrenamtlich tätigen Betreuers in der Betreuungsverfügung frei. ${ }^{820}$ Dem Einverständnis des Betreuten schreiben einige daher nur die Wirkung eines Indizes für das Vorliegen dieser gesetzlichen Kriterien zu. ${ }^{821}$ Diese Einschätzung wird dem Selbstbestimmungsrecht des Betroffenen nicht gerecht. Sachlich geht es bei der Frage der Vergütung um die Frage der Verwendung des Vermögens. \1901 Abs. 3 sichert die Beachtung der Wünsche des Betroffenen ${ }^{822}$ und somit auch die gewünschte Verteilung seines Vermögens. Der Betroffene bestimmt somit, was mit seinem Vermögen geschieht.

Die Festsetzung der Vergütung muss durch das Betreuungsgericht vorgenommen werden. ${ }^{823}$ Dies hat seinen Grund zum einen im Schutz des Betroffenen, der vor einer von dem Betreuer, entgegen oder ohne den Willen des Betroffenen,

\footnotetext{
815 Palandt/Diederichsen \1835 Rn. 1; MünchKommBGB/Wagenitz Vor \1835 R. 6; HKBUR/Bauer/Deinert \$1836 Rn. 37.

$816 \int 1835$ a ermöglicht hier statt einer Einzelabrechung auch eine Pauschalentschädigung. Die Vergütung wird allein durch das BGB geregelt, ein Verweis auf das VBVG liegt nicht vor (Palandt/Diederichsen $\int 1836$ Rn. 5).

817 Palandt/Diederichsen \1836 Rn. 6, 7; BT-Drucks. 15/4874 S. 32.

818 BayObLG FamRZ 2004, 1138, 1139; OLG Hamm FamRZ 2003, 116; Palandt/Diederichsen $\ 1836$ Rn. 6 ff.; Soergel/Zimmermann \1836 Rn. 35; Knittel \$1836 Rn. 48.

${ }^{819}$ Vgl. Dodegge/Roth C. Rn. 173.

820 So MünchKommBGB/Wagenitz, 』1836 Rn. 62, 63, 70 mit der Ausnahme, dass die unentgeltliche Besorgung der Geschäfte unzumutbar ist. Dann darf eine Vergütung zugebilligt werden. Palandt/Diedericbsen, 63. Auflage $\int 1836$ Rn. 25 billigt eine Vergütung aufgrund einer Vereinbarung immerhin dann, wenn der Betreute voll geschäftsfähig ist.

821 BayOLG FamRZ 2002, 130, 131. Wenn die Geschäftsfähigkeit des Betroffenen nicht in Frage steht weitergehend: Deinert/Lütgens Rn. 419 und Palandt/Diederichsen 63. Auflage \$1836 Rn. 25.

822 HK-BUR/Bauer \1901 Rn. 34 ff.; siehe auch \2 C. II., III.

823 Palandt/Diederichsen \1835 Rn. 7; MünchKommBGB/Wagenitz』1836 Rn. 75.
} 
selbstständig festgelegten und aus dem Vermögen des Betroffenen entnommenen Vergütung bewahrt werden soll. Zum andern erhält der Betreuer dadurch einen Vollstreckungstitel. ${ }^{824}$ Die Vergütung darf erst nach Beschluss durch das Gericht aus dem Vermögen des Betreuten entnommen werden. ${ }^{825}$ Die Einschaltung des Gerichts ändert schließlich aber nichts an der Grundnorm des $\ 1901$ Abs. 3, an die das Gericht wie der Betreuer gebunden ist. ${ }^{826}$ Das Ermessen des Gerichts nach $\int 1836$ Abs. 2 bezieht sich in diesem Fall also nicht auf die Frage von Umfang und Schwierigkeit der vormundschaftlichen Geschäfte, sondern die inhaltliche Überprüfung der Betreuungsverfügung mit Blick auf die Umsetzung des verfassten Willens. Daher ist die Ausgestaltung der Vergütung eines ehrenamtlich tätigen Betreuers eines vermögenden Betreuten nicht auf die Ausnahme des $\int 1836$ Abs. 2 beschränkt. Vielmehr ist die Vergütung - über diese gesetzlich festgelegte Ausnahme hinausgehend - der Gestaltung durch die Betreuungsverfügung eröffnet. .827

b) Individuelle Bestimmung der Vergütungshöhe

Die Höhe der nach \1836 Abs. 2 festgelegten Vergütung eines Berufsbetreuers galt nach einer in Rechtsprechung und Literatur bis zur Einführung des VGVB ${ }^{828}$ vertretenen Ansicht als Höchstgrenze für die Vergütung eines ehrenamtlich tätigen Betreuers. ${ }^{829}$ Grund war zum einen die Auffassung, dass der ehrenamtlich tätige Betreuer gerade nicht aus Gewinnerzielungsabsicht, sondern aus anderen Motiven handelt, er durch diese Finanzierung also nicht seinen Unterhalt bestreiten muss. ${ }^{830}$ Zum anderen sollte es zu keiner Kommerzialisierung der Vergütung kommen. Daher sei auch nur ein Aufwendungsersatz - als Ausgleich für die aufgewendete Zeit und aufgebrachte Leistung -, keine Vergütung gerechtfertigt, ${ }^{831}$ so dass die Vergütung eines Berufsbetreuers als Kontroll- und Höchstwert anzusehen sei. ${ }^{832}$ Die grundsätzlich ehrenamtliche und als staatsbürgerliche Ehrenpflicht folglich unentgeltlich zu führende Betreuung dürfe nur dann vergütet werden,

\footnotetext{
${ }^{824}$ MünchKommBGB/Wagenitz. 1836 Rn. 79.

825 Palandt/Diederichsen $\$ 1836$ Rn. 7.

826 Siehe hierzu \2 C. II.

827 Lipp FS Bienwald S. 177, 189 f.; Epple BWNotZ 1992, 27, 29.

828 01.07.2005.

${ }^{829}$ BayObLG FamRZ 2004, 1138, 1139; weniger streng (Kontrollwert): MünchKommBGB/Wagenitz $\ 1836$ Rn. 71.

830 BayObLG FamRZ 2004, 1138, 1139.

831 BayObLG FamRZ 2004, 1138, 1139.

832 Nicht in Form der Stundensätze als Mindestwerte (BayOLG FamRZ 2004, 1138, 1139). Anders: BayObLG FamRZ 2004, 1138, 1139; MünchKommBGB/Wagenitz. 1836 Rn. 71; weder Mindest- noch Höchstgrenze lt. Palandt/Diederichsen 63. Auflage \1836 Rn. 28; LG Baden-Baden 3 T 33/06, berichtigend jedoch OLG Karlsruhe $11 \mathrm{Wx}$ 74/06 am 01.03.2007; anders auch Bienwald FamRZ 2006, 1302.
} 
wenn ihre Ausübung als Ehrenpflicht nicht mehr zugemutet werden könne. ${ }^{833}$ Jüngere Entscheidungen zeigen zunehmend Kritik an dieser Grenzfestsetzung. Das neue System der Pauschalierung der Vergütung durch das VBVG erlaube keinen Vergleich der ehrenamtlichen mit der Berufsbetreuung, weil es sich nicht mehr am Zeitaufwand orientiere ${ }^{834}$.

Diese Grenze hat nur Bedeutung, wenn das Gericht - von sich aus oder auf Bestreben des Betreuers hin - eine objektiv angemessene Vergütung bewilligen muss. Dies ist bei der Frage der individuellen Vergütungsbestimmung durch die Betreuungsverfügung nicht der Fall. Hier bestimmt der zu Betreuende die Vergütung. Eine objektiv angemessene Grenze muss dann nicht durch das Betreuungsgericht bestimmt werden. Zudem kann eine objektive Grenze nicht vor dem (aktuellen) Wunsch oder den Äußerungen in Form einer Betreuungsverfügung des Betreuten gelten, weil dann \1901 Abs. 3 als Grundnorm außer Kraft gesetzt wäre.

Somit ist die Vergütung eines ehrenamtlich tätigen Betreuers über die Ausnahme des $\int 1836$ Abs. 2 hinaus der individuellen Gestaltungsmöglichkeit durch die Betreuungsverfügung eröffnet.

\section{Zwischenergebnis}

Bei der Festsetzung der Vergütung des Betreuers von vermögenden Betreuten ist zwischen beruflicher- und ehrenamtlicher Tätigkeit zu unterscheiden. Die Vergütung eines Berufsbetreuers ist in den $\iint 1836$, 1908i i.V.m. $\iint 4,5$ VBVG abschließend geregelt. Ein individueller Einfluss mittels der Betreuungsvergütung ist nicht möglich. Eine Festsetzung der Vergütung durch den Betroffenen ist nach $\int 1836$ Abs. 2 nur bei ehrenamtlich tätigen Betreuern möglich. Hier kann der vermögende Betroffene frei über sein Vermögen verfügen und eine individuelle, von den gesetzlichen Vorgaben abweichende Bestimmung mittels der Betreuungsverfügung treffen.

\section{Ergebnis zu $\$ 5$}

Der Einzelne kann durch antizipiert geäußerte Wünsche im Rahmen der Betreuungsverfügung umfassend auf die Führung seiner Betreuung einwirken. Die vom Gesetz in SS 1904 - 1908 vorgeschriebenen Genehmigungsvorbehalte des Betreuer sind zwingend und somit nicht durch den Betroffenen abdingbar. Wünsche über die Vornahme oder Nichtvornahme medizinischer Behandlungen sind gem. $\int 1901 \mathrm{a}$ Abs. 2 für den Betreuer verbindlich zu beachten. Eine Sterilisation nach \1905 kann durch antizipierten Entzug der Einwilligung verhindert werden. Eine

\footnotetext{
833 MünchKommBGB/Wagenitz』1836 Rn. 70, 71; Palandt/Diederichsen \1836 Rn. 2; Jurgeleit/Maier S 1836 Rn. 6.

834 OLG Karlsruhe 11 Wx 74/06 am 01.03.2007.
} 
Unterbringung ist nach \ 1906 Abs. 1 S. 1 nur zulässig, solange sie zum Wohl des Betreuten erforderlich ist. Dieser Verweis auf die allgemeine Regelung des \ 1901 Abs. 2 und 3 ermöglicht dem Betroffenen einen Einfluss auf die Frage der Erforderlichkeit. Durch die Definition seines Wohls mittels seiner Wünsche und Vorstellungen in der Betreuungsverfügung bestimmt der Einzelne mittelbar die Anforderungen seiner Unterbringung. Gleiches gilt für die in \1907 regulierte Aufgabe seiner Mietwohnung und die Ausstattung gem. \1908. In der Verwaltung seines Vermögens wird der Betroffene durch \1908i Abs. 2 i.V.m. \ 1804 eingeschränkt. V.a. die Regelung zum geltenden Schenkungsverbot ist teleologisch zu reduzieren. Im Übrigen ist der Betroffene frei in der Ausgestaltung seiner Betreuung. 


\section{\$6 Missachtung der Betreuungsverfügung}

\section{A. Durch das Gericht}

I. Missachtung der Wunschbefolgungspflicht

Die Missachtung der Vorgaben der Betreuungsverfügung durch das Gericht ist unterschiedlich zu bewerten: Missachtet das Gericht die Betroffenenwünsche, die gegen gesetzliche Regelungen, wie einem Genehmigungserfordernis oder andere zwingende Regeln verstoßen, ist dies rechtmäßig. Denn diese Betroffenenwünsche sind für das Betreuungsgericht und den Betreuer nicht verbindlich. ${ }^{835}$ Diese Wünsche müssen im Rahmen der Umdeutung und Auslegung der Betreuungsverfügung zumindest dem Sinn nach in der gerichtlichen Entscheidung beachtet werden. ${ }^{836}$ Missachtet das Gericht die Wünsche, weil bei ihrem Befolgen eine konkrete ${ }^{837}$ Gefährdung des Betroffenenwohls entstünde, ist die Entscheidung ebenfalls rechtmäßig, da sie $\ 1901$ Abs. 2 und 3 entspricht. Dabei sind die Vorgaben der Betreuungsverfügung zwar verbindlich, im konkreten Fall ,darf das Betreunngsgericht ibnen jedoch nicht nachkommen ' ${ }^{\circ 38}$. Trotz Nichtbeachtung der Verfügung im Rahmen

\footnotetext{
835 Siehe oben \ 2 C. III. 3.

836 BayObLG FamRZ 1994, 323, 324.

837 Bienwald/Sonnenfeld/Hoffmann \ 1897 Rn. 59 f.

${ }^{838}$ MünchKommBGB/Schwab S 1897 Rn. 23 und S 1901 Rn. 14.
} 
der Entscheidungsfindung handelt das Gericht daher pflichtgemäß und somit rechtmäßig. Begründet sich das Missachten hingegen in einer falschen Gesetzesanwendung, begeht das Gericht einen Rechtsfehler. Gleiches gilt für alle Fragen im Betreuungsverfahren, der Auswahl und der Überwachung des Betreuers, in denen das Gericht die gem. \1901 Abs. 3 S. 1 HS 1 verbindlichen Wünsche des Betroffenen nicht ausreichend beachtet. Legt das Gericht das Gesetz falsch aus oder wendet das Recht falsch an, hat dies in der Phase der Entscheidungsfindung keine direkten Auswirkungen. Mit einer Einwendung kann ein Verfahrensbeteiligter das Gericht jedoch auf seine (eintretende) falsche Gesetzesanwendung hinweisen.

\section{Rechtsfolgen und Beschwerdeberechtigung}

Trifft das Betreuungsgericht eine Entscheidung nach $\iint$ 286, 38 FamFG/ $\int 69$ FGG in der Sache, wird diese wirksam. Dies gilt unabhängig davon, ob sie materiell und formell rechtmäßig ist. ${ }^{839}$ Die Entscheidung über die Bestellung des Betreuers, den Umfang, Inhalt oder Bestand der Betreuung kann jedoch gem. \\303 ff., 58 ff. FamFG/ \19 FGG mit der Beschwerde nachgeprüft werden. Diese hat Erfolg, wenn sie zulässig und begründet ist. Seit dem 3. BtÄndG ist diese gem. \63 Abs. 1 FamFG in einer Frist von einem Monat einzulegen. 840

Ist die Beschwerde begründet, trifft die Beschwerdeinstanz eine Entscheidung in der Sache. ${ }^{841}$ Die Ermessensentscheidung kann vom Rechtsbeschwerdegericht auf Rechtsfehler überprüft werden. ${ }^{842}$ Die Ausübung ist fehlerhaft, wenn der Tatrichter sich des ihm zustehenden Ermessens nicht bewusst ist, nicht alle wesentlichen Umstände berücksichtigt, von dem Ermessen in einer dem Zweck der Ermächtigung nicht entsprechenden Weise Gebrauch macht oder die gesetzlichen Ermessensgrenzen überschreitet. ${ }^{843}$

Missachtungen der gesetzlichen Vorgaben im Verfahren der Betreuerbestellung können unterschiedlich sein. Die Bestellung des Verfahrenspflegers durch den Richter kann von den Beteiligten gem. \276 Abs. 6 FamFG nicht selbstständig angefochten werden, weil es sich nur um eine Zwischenentscheidung han-

\footnotetext{
${ }^{839}$ Lipp FS Bienwald S. 177, 186; Perau MittRhNotK 1996, 285, 289; Keidel/Kuntze/Winkler/Kayser §69 g FGG Rn. 13.

${ }^{840}$ Nach dem bis zum 31.08.2009 geltenden \69 FGG war die Beschwerde unbefristet. Das Beschwerderecht unterlag nur der Verwirkung (Keidel/Kuntze/Winkler/Kabl\$19 FGG Rn. 52; Keidel/Kuntze/Winkler/Sternal \$ 21 FGG Rn. 41 ff.).

841 Zum Recht des FGG: Pawlowski/Smid Rn. 791.

842 MünchKommBGB/Schwab \$ 1908 b Rn. 8; BayObLG FamRZ 1996, 1105, 1106; BayObLG FamRZ 1999, 1169, 1170; Nicht auf Zweckmäßigkeit MünchKommBGB/Schwab \$ 1897 Rn. 26. 843 MünchKommBGB/Schwab \& 1897 Rn. 26; LG Stendal FamRZ 1995, 507, 508. Die Ermessensausübung ist im Rechtsbeschwerdeverfahren nur beschränkt nachprüfbar (Bienwald/Sonnenfeld/Hoffmann \ 1897 Rn. 73; BayObLG FamRZ 1994, 530; OLG Karlsruhe BtPrax 1994, 214). Eine Nachprüfung kann jedoch mittels Anfechtung der Entscheidung wegen eines Verfahrensfehlers erfolgen.
} 
delt. ${ }^{844}$ Eine Anfechtung der Endentscheidung mit der Begründung, die Bestellung des Verfahrenspflegers sei fehlerhaft und mit auch die Endentscheidung, ist hingegen möglich.

Versäumt das Gericht pflichtwidrig, den Betroffenen in seiner gewohnten Umgebung anzuhören, verletzt es die ihm obliegende Amtsermittlungspflicht gem. IS 26, 278 Abs. 1 - 3, 34 FamFG. ${ }^{845}$

Folgt das Gericht dem Vorschlag des Betroffenen im Verfahren der Betreuerbestellung in Bezug auf seinen Wunschbetreuer nicht, ohne dass dadurch eine konkrete Gefahr für den Betroffenen abgewendet werden würde, bzw. Anhaltspunkte für die Gefährdung vorlagen, übergeht es den nach \1897 Abs. 4 verbindlichen Vorschlag des Betroffenen unrechtmäßigerweise. Dann liegt die Pflichtverletzung durch das Betreuungsgericht in dem Nichtbefolgen des Betroffenenvorschlags. Die Beschwerde kann sich dann auf die Betreuerauswahl beschränken. ${ }^{846}$ Ziel der Beschwerde kann dann die Ernennung des Beschwerdeführers selbst ${ }^{847}$ bzw. die Ernennung einer anderen als die ernannte Person sein. ${ }^{848}$ Soll die Entscheidung des Gerichts mittels der Beschwerde geprüft werden, stellt sich die Frage der Beschwerdeberechtigung. Dabei ist danach zu differenzieren, ob die Betreuung von Amts wegen oder auf Wunsch des Betroffenen eingerichtet wurde.

\section{Einrichtung der Betreuung von Amts wegen}

Wurde die Betreuung gem. \1896 Abs. 1 S. 1 HS 2, 1. Alt. von Amts wegen angeordnet, waren nach dem alten Recht gem. \69g Abs. 1 FGG nur diejenigen beschwerdeberechtigt, deren subjektives Recht gem. \ 20 FGG durch die Betreuerbestellung beeinträchtigt wurde, sowie die in $\int 69 \mathrm{~g}$ Abs. 1 FGG Genannten und gem. \67 Abs. 2 FGG der Verfahrenspfleger. Danach stellte sich die Frage, ob der Wunschbetreuer des Betroffenen - bei Nichternennung zum Betreuer - in diesen Berechtigtenkreis fiel. ${ }^{849}$ Eine Beschwerde aus \69g Abs. 1 FGG konnte nach gefestigter Rechtsprechung dabei auf die Auswahl des Betreuers begrenzt werden. ${ }^{850}$

\footnotetext{
844 Jürgens/Kröger/Marschner/Winterstein Rn. 351 (mehr dazu dort); BGH BtPrax 2003, 266; BayObLG BtPrax 1998, 148; OLG Frankfurt a.M. BtPrax 2001, 207, 208; OLG Stuttgart FamRZ 2001, 39; KG FamRZ 1996, 357, 358.

845 OLG Düsseldorf FamRZ 1996, 1373.

846 Lipp FS Bienwald S. 177, 187; BGH NJW 1996, 1825 = FamRZ 1996, 607; HK-BUR/Bauer

\1897 Rn. 8b; Keidel/Kuntze/Winkler/Kayser \69g FGG Rn. 13; Rieger FS Schwab S. $1043,1052$.

847 Staudinger/Bienwald \1896 Rn. 205.

848 Rieger FS Schwab S. 1043, 1051.

849 Bejahend: OLG Oldenburg FamRZ 1995, 432; Rieger FS Schwab S. 1043, 1053 ff.; verneinend: BayObLG FamRZ 2003, 1219, 1220; HK-BUR/Bauer \69 FGG Rn. 16 .

850 BT-Drucks. 11/4528 S. 120; BGH NJW 1996, 1825; KG Berlin FamRZ 1995, 1442; OLG Schleswig FamRZ 1995, 432; HK-BUR/Bauer \$ 1897 Rn. 8a; Rieger FS Schwab S. 1043, 1052; Palandt/Diederichsen 55. Aufl. \1897 Rn. 22; anderer Ansicht: Kemper FuR 1994, 267, 269.
} 
Ein Beschwerderecht aus \69g Abs. 1 FGG lehnte die herrschende Meinung für einen Dritten (den Wunschbetreuer) ab, 851 wenn dieser nicht der Ehegatte oder Lebenspartner des Betroffenen, mit diesem in erster Linie verwandt oder verschwägert oder in der Seitenlinie bis zum dritten Grad verwandt ist. Auch die Beschwerdeberechtigung des Dritten aus \59 FamFG/ \20 Abs. 1 FGG wird nach überwiegender Meinung verneint, weil durch die Bestellung eines (anderen) Betreuers kein subjektives Recht des in der Betreuungsverfügung benannten Wunschbetreuers verletzt werde. ${ }^{852}$

Durch die aktuellen Regelungen wurde neben dem gem. \59 FamFG/ \ 20 FGG in seinen Rechten Betroffenen der Kreis der Beschwerdeberechtigten in $\int 303$ FamFG erweitert. ${ }^{853}$ \& 303 FamFG ist die Nachfolgeregelung des \69f FGG. Gem. \303 Abs. 2 Nr. 2 FamFG kann auch die Vertrauensperson des Betreuten im Interesse des Betroffenen Beschwerde einlegen. Diese Möglichkeit steht jedoch unter der Voraussetzung, dass die Vertrauensperson im ersten Rechtszug beteiligt worden ist. In der Frage nach der Beschwerdeberechtigung des nicht zum Betreuer ernannten Wunschbetreuers des Betroffenen liegt eine Beteiligung im ersten Rechtszug gerade nicht vor. Nach dem Gesetzestext kann eine Beschwerdeberechtigung der Vertrauensperson dann nicht angenommen werden. Nach der Gesetzesbegründung sollen durch die Voraussetzung der Verfahrensbeteiligung in erster Instanz Beschwerden solcher Angehöriger vermieden werden, die am Verfahren erster Instanz kein Interesse gezeigt haben. ${ }^{854}$ Dieser Gedanke wird auf die Vertrauensperson zu übertragen sein. Der Wunschbetreuer fällt unter Vertrauensperson des Betreuten. Der Wunschbetreuer wird nicht aus fehlendem Interesse sondern aufgrund fehlender Information Verfahrensunbeteiligter gewesen sein. Seine fehlende Beteiligung in erster Instanz und seine Beschwerdeberechtigung liegen im Interesse des Betroffenen. Die gesetzliche Formulierung des $\int 303$ Abs. 2 Nr. 2 FamFG ist daher entweder dahin umzudeuten, dass der nicht ernannte Wunschbetreuer ebenfalls beschwerdeberechtigt ist oder aber ihm ein Beschwerderecht aus $\int 59$ FamFG zugesprochen wird. Dies lässt sich durch die übrige gesetzliche Regelung begründen und soll im Folgenden aufgezeigt werden.

\section{a) Gesetzesbegründung}

Laut der Gesetzesbegründung zu \69g FGG sei ein Antragsrecht auf die Übernahme der Betreuung für Dritte mit ,dem Wesen der Betreunng als eine auf das Wobl des Betroffenen abzielende öffentliche Hilfe" nicht vereinbar. ${ }^{855}$ Vielmehr sei das Interesse

\footnotetext{
851 BGHZ 132, 157, 160 m.w.N.

852 Ausdrücklich Staudinger/Bienwald S 1897 Rn. 210; BGH FamRZ 1989, 369, 370; so wohl auch Dodegge/Roth A. Rn. 187; HK-BUR/Bauer \69f FGG Rn. 19; anderer Ansicht: OLG Zweibrücken FamRZ 2003, 703; Zimmermann S. 214 (a.F.).

853 Dabei wurde aber der Kreis der Verwandten, die am Verfahren beteiligt werden können gegenüber \69g FGG eingeschränkt, vgl. BT-Drucks. 16/6308 S. 271.

854 BT-Drucks. 16/ 6308 S. 271 f.

855 BT-Drucks. 11/4528 S. 117.
} 
Dritter durch das in $\int 69 \mathrm{~g}$ FGG enthaltene Beschwerderecht der dort aufgeführten Dritten ausreichend gewahrt. Diese Begründung bedenkt die Möglichkeit des Betroffenen, seinen Wunschbetreuer gem. \1897 Abs. 4 antizipiert zu bestimmen nicht ausreichend. Erst durch die gesetzlich geschaffene Möglichkeit, in einer Betreuungsverfügung die eigenen Wünsche antizipiert und verbindlich zu äußern, kann die Situation entstehen, dass der Wunschbetreuer gerade nicht zu dem in \303 FamFG/ \69g FGG genannten Personenkreis gehört. Es ist davon auszugehen, dass der Betroffene aus bestimmten Überlegungen seinen Wunschbetreuer nicht aus diesem Personenkreis ausgewählt hat, sei es zur Vermeidung von Konfliktsituationen in der Familie oder aus anderen Gründen. ${ }^{856}$ Kann sich der Wunschbetreuer jedoch nicht gegen die dem Willen des Betreuten entgegenstehende Betreuerwahl wenden, wird durch diese gesetzliche Regelung gerade das Gegenteil von dem ursprünglich gesetzlichen Ziel der Betreuung erreicht. Der Wille des Betroffenen wird bzw. wurde in diesem Fall nicht umgesetzt und kann aufgrund fehlender Beschwerdebefugnis von dem Wunschbetreuer nicht geltend gemacht werden. Nach den gesetzgeberischen Zielen müsste der Wunschbetreuer daher ein Beschwerderecht erhalten.

b) Beschwerderecht des abgelösten Betreuers

Bei einem regulären Betreuerwechsel bei Aufhebung einer Betreuung nach \1908b kann der (alte) Betreuer grundsätzlich keine Beschwerde gegen die Aufhebung einlegen, da in diesem Fall ausschließlich die Interessen des Betreuten und nicht die des Betreuers maßgeblich seien. ${ }^{857}$ Nach einem Betreuerwechsel gegen den Willen des Betreuers ${ }^{858}$ kann der abgelöste Betreuer jedoch im eigenen Namen Beschwerde nach \59 FamFG einlegen. ${ }^{859}$ Dies ist darin begründet, dass der ehemalige Betreuer einen Anspruch darauf haben soll, nicht ohne gesetzlichen Grund seines Amtes enthoben zu werden. ${ }^{860}$ Die Begründung eines solchen Anspruchs ist für sich gesehen interessensgerecht. Sie verschiebt aber die von der herrschenden Meinung befürwortete grundsätzliche Interessensverteilung in der Betreuung zugunsten des Betreuten. Ein derart begründetes Beschwerderecht steht allein im Interesse des abgelösten Betreuers.

Im Vergleich zum Beschwerderecht des Wunschbetreuers entsteht bei ähnlicher Sachlage eine vergleichbare Verschiebung der Interessen. Der Wunschbetreuer hat nach der herrschenden Meinung bei fehlender unbegründeter Benennung nicht die Möglichkeit, einen dem unwillentlich entlassenen Betreuer aus $\int 59$ FamFG äquivalenten Anspruch zu erwerben. Ihm steht kein vergleichbarer

\footnotetext{
856 So auch schon Müller-Freienfels FS Coing S. 395, 402 zur Auswahl des Pflegers durch den Prinzi-

pal; darüber herrscht aktuell Einigkeit, vgl. nur Zimmermann Rn. 370 (a.F.).

857 Keidel/Kuntze/Winkler/Kayser \ 69g FGG Rn. 23.

858 OLG Köln FamRZ 1997, 1293.

859 OLG Köln FamRZ 1997, 1293; Keidel/Kuntze/Winkler/Kayser \ 69g FGG Rn. 23; HK-

BUR/Bauer \69f FGG Rn. 93; Bienwald/Sonnenfeld/Hoffmann \69 FGG Rn. 51.

860 OLG Braunschweig DAVorm 1993, 991.
} 
Anspruch zu. Er hatte im Gegensatz zum abgelösten Betreuer das Amt des Betreuers noch nicht inne, so dass er folglich keinen Anspruch auf begründete Amtsenthebung haben kann, jedoch ist zu überlegen, ob er einen Anspruch auf begründete Nichtbenennung haben sollte. Dies wäre nicht aus seinen Rechten, sondern vielmehr aus dem Willen des Betreuten nach \1897 Abs. 4 S. 1 und der Interessensausrichtung der Betreuung auf die Wünsche und das Wohl des Betreuten nach $\int 1901$ Abs. 3 herzuleiten.

\section{c) Rechtsinstitut Vorsorgevollmacht}

Auch der Vorsorgebevollmächtigte hat kein Beschwerderecht, wenn er nicht zum Personenkreis des \303 FamFG/ \69g Abs. 1 FGG gehört und trotz Bestehens einer wirksamen Vollmacht eine Betreuung eingerichtet wurde und er nicht zum Betreuer ernannt wurde. Ein eigenes Beschwerderecht aus \59 FamFG bestehe nicht, weil durch die Betreuerbestellung keine subjektive Rechtsverletzung des Bevollmächtigten vorliege. ${ }^{861}$ Dieser leitet seine Rechtsstellung ausschließlich aus fremdem Recht, d.h. der Vollmacht ab. Diese begründe kein eigenes subjektives Recht, weil sie jederzeit widerrufen werden könne und daher keine gesicherte Rechtsposition entstünde. ${ }^{862} \mathrm{Ihr}$ liege nur das subjektive Interesse des Betroffenen zugrunde. Der Bevollmächtigte könne aber immer im Namen des Betreuten Beschwerde gem. \59 FamFG einlegen.863 Ein Beschwerderecht aus \59 FamFG entstünde nur, wenn ein Kontrollbetreuer bestellt werde, ${ }^{864}$ da dann in geschützte Rechte des Bevollmächtigten eingegriffen werde.

Die Gegenansicht gewährt dem Bevollmächtigten ein eigenes Beschwerderecht aus subjektiver Rechtsverletzung durch unbegründete Betreuerbestellung. ${ }^{865}$ Diese schöpfe sich aus dem der Vollmacht zugrunde liegenden Rechtsverhältnis, in das durch die Einrichtung einer Betreuung unmittelbar eingegriffen werde. ${ }^{866}$

Die Bevollmächtigung dient der Durchsetzung und Berücksichtigung der Wünsche und Vorstellungen des Vollmachtgebers und soll nicht die Wahrnehmung einer Rechtsmacht im Interesse des Bevollmächtigten ermöglichen. ${ }^{867}$ Fraglich ist, ob dem eigenen Rechte und Befugnisse des Bevollmächtigten entgegen-

\footnotetext{
861 Staudinger/Bienwald \$ 1897 Rn. 155; Keidel/Kuntze/Winkler/Kayser \69g FGG Rn. 23; BayObLG FamRZ 2003, 1219; Bienwald/Sonnenfeld/Hoffmann \69g FGG Rn. 20; Palandt/Heinrichs Vor \ 164 Rn. 5; MünchKommBGB/Schramm \164 Rn. 69; OLG Stuttgart FamRZ 1995, 427; BayObLG FamRZ 2003, 1219; Anderer Ansicht: OLG Zweibrücken FamRZ 2003, 703; HK-BUR/Bauer \69f FGG Rn. 16a; BayObLG FamRZ 2001, 453, 454 m.w.N.; Staudinger/Schilken (13. Auflage) Vor $\$ 164$ Rn. 16 f. 
stehen. ${ }^{868}$ Diesbezüglich ist zu bedenken, dass auch ,fremdnützig gebraucbte Recbte eigene Recbte "sein können. ${ }^{869}$ Demnach würde der Vorsorgebevollmächtigte eigene Rechte aus einer fremdnützigen Position ableiten.

Der Vorsorgebevollmächtigte wird rein privatautonom durch den Vollmachtgeber ernannt. Mittels der Betreuungsverfügung übt der Betroffene im Rahmen der gesetzlich geregelten Betreuung seine Privatautonomie aus. Die Betreuungsverfügung formuliert er auch unter dem Gesichtspunkt, dass der Betreuer dem Betreuungsgericht gegenüber zur Rechenschaft verpflichtet ist und dessen Kontrolle unterliegt. Weil der Bevollmächtigte grundsätzlich im Namen des Betroffenen Beschwerde einlegen kann, steht die privatautonom ausgestaltete Vorsorgevollmacht in dem Fall, dass der Wunschbetreuer nicht zum Betreuer bestellt wurde und im Interesse des Betroffenen dagegen vorgehen möchte, der Rechtsweg der Beschwerde offen. Die der gerichtlichen Kontrolle unterlegenen und als staatliche Hilfe zu verstehende Betreuung, in deren Mittelpunkt die Wünsche und das Wohl des Betroffenen stehen sollen, versperrt den Rechtsweg der Beschwerde. Der Wunsch des Betroffenen, den von ihm ausgewählten Betreuer zu bestellen, kann in diesem Fall nicht gerichtlich durchgesetzt werden. Seine Nennung in der Betreuungsverfügung muss für den Wunschbetreuer daher - im Vergleich zur Vorsorgevollmacht - entweder als Vollmacht zur Beschwerde im Namen des Betroffenen gelten, oder aber es muss ihm ein eigenes Beschwerderecht aus subjektiver Rechtsverletzung aufgrund fehlender Teilnahme an der Betreuerbestellung zugesprochen werden.

Bei Kongruenz von Aufgabenkreis des trotz einer bestehenden Vollmacht bestellten Betreuers und dem Umfang der Vollmacht wird durch die Einrichtung der Betreuung in das der Vollmacht zugrunde liegende Rechtsverhältnis eingegriffen, so dass dem Bevollmächtigten in diesem Fall ausnahmsweise ein Beschwerderecht aus $₫ 59$ FamFG aus subjektiver Rechtsverletzung entsteht. Diese Annahme widerspricht der grundsätzlichen Interessenverteilung der Vollmacht, wonach sich nach herrschender Ansicht die Aufgabe des Bevollmächtigten in der Geltendmachung der (für ihn fremden) Rechte des Betroffenen erschöpft. ${ }^{870}$ Eine Vorsorgevollmacht wird im Rahmen von Wirksamkeitszweifel gem. \140 in eine Betreuungsverfügung umgedeutet, so dass der eigentliche Bevollmächtigte als Wunschbetreuer benannt wird. Sollte diese Umdeutung misslingen und der ursprünglich Bevollmächtigte nicht einmal zum Betreuer bestellt werden, steht ihm nach den derzeitigen gesetzlichen Regelungen kein Beschwerderecht zu, wenn er nicht gem. \303 FamFG in erster Instanz beteiligt war. Nach einer solchen Umdeutung kann der ursprünglich Bevollmächtigte auch nicht mehr im Namen des Vollmachtgebers (dann: Betreuten) Beschwerde einlegen. Im Interesse des Vollmachtgebers ist dem Vollmachtnehmer hier also auch ein Beschwerderecht gem. \59 FamFG aus

\footnotetext{
868 So auch: Rieger FS Schwab S. 1043, 1053.

869 Rieger FS Schwab S. 1043, 1053; MünchKommBGB/Schwab \1896 Rn. 208 Fn. 537.

870 Staudinger/Bienwald \$1897 Rn. 155.
} 
subjektiver Rechtsverletzung aus der Nennung in der Vorsorgevollmacht zuzuschreiben. Aufgrund der möglichen Umdeutung einer Vorsorgevollmacht in eine Betreuungsverfügung und dem Betroffenenwunsch, kann ein solches Recht auch für den Wunschbetreuer hergeleitet werden.

d) $\iint 1776,1779$ und Art. 6 Abs. 1 GG

Die SS 1776, 1779 enthalten ein Vorschlagsrecht der Eltern für eine mögliche postmortale 871 Übertragung der Vormundschaft. Danach ist ,als Vormund [...] berufen, wer von den Eltern des Mündels als Vormund benannt ist", vgl. \1776 Abs. 1. Im Gegensatz zur vorliegenden Situation schlägt daher nicht der Betroffene selber für die mögliche spätere Situation der eigenen Unfähigkeit einen Vertreter vor. Vielmehr befindet sich der schutzwürdige Betroffene - das Mündel - noch in der hilflosen Situation, vertreten werden zu müssen. Es ist gem. \104 geschäftsunfähig. Daher kann es kraft Gesetz nicht für sich handeln. Für eine mögliche Vormundschaft können die Eltern als Interessenswahrer des Mündels einen Wunschvormund benennen, vgl. S 1776. Dieses gewährt dem benannten Wunschvormund nach herrschender Meinung ein subjektiv öffentliches Recht gegen den Staat aus $\int 1778$ Abs. 4 auf Bestellung zum Vormund einerseits ${ }^{872}$ und Beachtung dieser Rechtsstellung als Vormund andererseits, so dass die Bestellung eines Mitvormundes nicht ohne Verletzung der Rechte des Wunschvormundes möglich ist. 873 Sollte der Wunschvormund unbegründet nicht zum Vormund benannt werden, hat das Gericht gegenüber dem Mündel die Pflicht, den bestellten Vormund zu entlassen und den Vorgeschlagenen zu ernennen. Der Benannte darf ohne seine Zustimmung nur in den in \1778 gesetzlich abschließend geregelten Fällen ${ }^{874}$ übergangen werden: wenn er unfähig, ${ }^{875}$ untauglich, ${ }^{876}$ verhindert oder verzögert ist, Interessen des Mündels gefährden oder das über 14jährige Mündel widersprechen würde. Der Vorgeschlagene ist jedoch zur Übernahme erst nach gerichtlicher Ernennung verpflichtet. ${ }^{877}$ Die Ernennung eines Nicht-Benannten ist grundsätzlich wirksam. ${ }^{878}$ Die Auswahl des Dritten ist durch jeden, ,der ein berechtigtes Interesse hat" anfechtbar. Das aus dem Benennungsrecht der Eltern erwachsene subjektive

\footnotetext{
871 MünchKommBGB/Wagenitz $\int 1776$ Rn. 8.

872 Staudinger/Engler S 1778 Rn. 5; MünchKommBGB/Schwab \ 1778 Rn. 1; Gernhuber/CoesterWaltjen S 70 Rn. 34, 37. Wenn auch nur als „Recht auf Bestellung“ definiert, wird dies wohl auch als subjektives Recht verstanden, da bei Übergehen sofortige Beschwerde nach \60 Abs. 1 Nr. 1 FGG eingelegt werden kann (Palandt/Diederichsen \1776 Rn. 2; Dölle \ 120 III; BayObLG FamRZ 1966, 323, 324 f.). So gilt als Berufener im Sinne von \ 1776 neben dem nach dem mutmaßlichen oder lediglich wahrscheinlichen Willen der Eltern Ernannte, nicht hingegen der in der letztwilligen Verfügung Genannte (Palandt/Diederichsen \1779 Rn. 7).

873 MünchKommBGB/Wagenitz \1778 Rn. 1.

874 Gernhuber/Coester-Waltjen \ 70 Rn. 37; MünchKommBGB/Wagenitz S 1778 Rn. 6.

875 S 1778 Abs. 1 Nr. 1 i.V.m. \ 1780.

876 \ 1778 Abs. 1 Nr. 1 i.V.m \ 1781.

877 Gernhuber/Coester-Waltjen S 70 Rn. 37; Rauscher Rn. 1204; Palandt/Diederichsen S 1776 Rn. 2.

878 Soergel/Zimmermann S 1776 Rn. 8 und S 1778 Rn. 10.
} 
Recht des Benannten sichert dem Benannten bei Übergehen ein Recht zur Beschwerde gem. \59 FamFG. ${ }^{879}$ Dann ist er an Stelle des Vormundes zu bestellen. Dies sogar unabhängig davon, ob dem Gericht seine Benennung bekannt war. ${ }^{880}$ Der als Vormund Vorgeschlagene kann also aus eigenem Recht gegen die Bestellung eines anderen Vormundes Beschwerde einlegen.

$\int 1908$ i verweist als Schaltnorm für das Betreuungsrecht auf einige Vorschriften des Vormundschaftsrechts. Der Gesetzgeber schuf mit dem Betreuungsrecht bewusst einen Regelungsbereich, der nur den Personenkreis der Volljährigen und nicht auch den der Minderjährigen betrifft. Eine globale Verweisung auf die Vorschriften der Vormundschaft wurde vermieden. Weil das Recht der Erwachsenen grundsätzlich separat geregelt und das Rechtsinstitut der Entmündigung abgeschafft werden sollte, werden in \1908i Abs. 1 nur Einzelvorschriften des Vormundschaftsrechts benannt. ${ }^{881}$ Die Grundgedanken des Betreuungsrechts sind bei der Auslegung der vormundschaftlichen Normen zu beachten. ${ }^{882}$ Das Benennungsrecht ist Ausfluss der elterlichen Sorge und gilt daher nicht für die rechtliche Betreuung. ${ }^{883}$ In der Literatur ${ }^{884}$ wird ausdrücklich der Vergleich zwischen Vormundschafts- und Betreuungsrecht gezogen und auf das Vorschlagsrechts in \$S 1897 Abs. 4, 1901 Abs. 2, 3 verwiesen. Es gebe keinen Anhaltpunkt dafür, diese Grundstruktur nicht im Betreuungsrecht anzuwenden ${ }^{885}$ und dem in der Betreuungsverfügung benannten Wunschbetreuer aus dieser Benennung ein subjektives Rechts zuzusprechen. Eine Beschwerde des nicht zum Betreuer bestellten wäre dann gem. \59 Abs. 1 FamFG möglich. Dieser Schluss erscheint zwingend, bedenkt man, dass der Vorschlag im Vormundschaftsrecht von Dritten - nämlich den Eltern - und im Betreuungsrecht von dem Betroffenen selbst kommt. Der Zusammenhang wäre daher im Betreuungsrecht erst recht anzunehmen.

Diskutiert wurde ein Beschwerderecht aus Art. 6 Abs. 1 GG. ${ }^{886}$ Dies würde ein Beschwerderecht naher Angehöriger und der Personen begründen, die eine bestehende Bindung zum Betroffenen haben, ${ }^{887}$ es verhilft aber nicht dem Wunsch des Betroffenen zur Geltung. Ein Beschwerderecht für den Wunschbetreuer ist aus Art. 6 Abs. 1 GG daher nicht abzuleiten. ${ }^{888}$

\footnotetext{
879 Soergel/Zimmermann \1776 Rn. 7; Gernhuber/Coester-Waltjen \70 Rn. 39; Münch-

KommBGB/Wagenitz: 1777 Rn. 10.

880 Palandt/Diederichsen \1778 Rn. 1.

881 Bienwald/Sonnenfeld/Hoffmann \1908i Rn. 4; BT-Drucks. 11/4528 S. 159.

882 BT-Drucks. 11/4528 S. 159.

883 Soergel/Zimmermann \$1776 Rn. 3.

884 Soergel/Zimmermann \1776 Rn 2, 8; ausführlich: Staudinger/Bienwald \1897 Rn. 47.

885 BGH BtPrax 1992, 28, 29.

886 Vgl. nur BVerfGE 33, 236 ff.: Bei der erstmaligen Bestellung eines Gebrechlichkeitspflegers wurde ein Beschwerderecht aus Art. 6 Abs. 1 GG angenommen.

887 Staudinger/Bienwald \$ 1897 Rn. 47; BVerfGE 68, 176.

888 BGHZ 132, 157, 162 f.; Staudinger/Bienwald \$ 1897 Rn. 47.
} 
e) Zwischenergebnis

Das Amt des Betreuers ist ein Ehrenamt mit vielen Pflichten und wenigen Rechten. ${ }^{889}$ Wer entgegen seinem Willen als Betreuer entlassen wird, hat einen Anspruch auf Begründung. Der Wunschbetreuer hat (noch) kein Amt inne. ${ }^{890}$ Jedoch obliegt ihm bereits die Pflicht, als von dem Betroffenen ausgewählte Person nach \ 1897 Abs. 4 das Amt des Betreuers nach \ 1898 Abs. 1 zu übernehmen, soweit keine der gesetzlichen Ausnahmen vorliegt. Mit der Nennung des Wunschbetreuers in der Betreuungsverfügung formuliert der Betroffene eine Übernahmepflicht die mit Einleitung des Verfahrens konkret entsteht.

Das Verfahrens- und Betreuungsrecht stellt den Betroffenen und seine Wünsche und Interessen immer in den Mittelpunkt, vgl. nur $\iint 5$ 275, 278 FamFG und

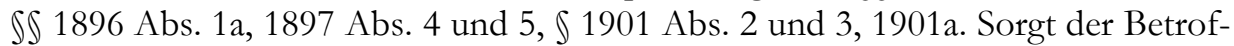
fene für eine mögliche Betreuung mittels der Nennung eines Wunschbetreuers in der Betreuungsverfügung vor, ist sein Wunsch bekannt. Wird sein Vorschlag durch das Betreuungsgericht missachtet, kann der Betreute nach \59 FamFG gegen diese Entscheidung mittels der Beschwerde vorgehen. Problematisch wird dies, wenn er dazu nicht mehr in der Lage ist. Dann ist es Aufgabe des Verfahrenspflegers, die Rechte und Wünsche des Betreuten geltend zu machen und die Betreuerauswahl zu rügen, vgl. \276 Abs. 2 FamFG. Ein Verfahrenspfleger wird jedoch nicht in jedem Fall bestellt, vgl. \276 FamFG. Greift dieser Schutz für die Rechte und Interessen des Betroffenen nicht, bleibt die Möglichkeit für den amtierenden Betreuer nach \59 FGG das Recht des Betroffenen zur Wunschbefolgung aus $\int 1897$ Abs. 4 zu rügen. Eine solche Pflicht ist gesetzlich nicht ausdrücklich normiert. Sie ist jedoch aus der Wunschbefolgungspflicht des $\int 1897$ Abs. 4 i.V.m. \ 1901 Abs. 2, 3 und \ 1901a abzuleiten. Der ernannte Betreuer wird dies jedoch nicht tun, weil er dann selbst entlassen wird.

Der Wunschbetreuer hat nach herrschender Meinung durch die Nennung in der Betreuungsverfügung keine eigene Rechtsposition. Indem das Gericht ihn nicht ernennt, missachtet es den Betroffenenwunsch. Diese wirksame Entscheidung des Gerichts kann durch den nicht zum Betreuer ernannten Wunschbetreuer nicht gerügt werden. Diese Situation widerspricht dem gesetzgeberischen Ziel, die Rechte und Wünsche des Betreuten in den Mittelpunkt des Betreuungsrechts zu stellen.

Die zusätzliche Bevollmächtigung des in der Betreuungsverfügung genannten Wunschbetreuers, ermöglicht ihm nach \59 FamFG als Bevollmächtigter, die Betreuerauswahl zu rügen. ${ }^{891}$ Diese Möglichkeit eröffnet das Gesetz in seiner der-

889 Vgl. nur \1898 Abs. 1 - Pflicht zur Übernahme der Betreuung; \1901 Abs. 2 - Pflicht, die

Angelegenheiten des Betreuten so zu besorgen, wie es dessen Wohl entspricht; $\ 1901$ Abs. 3 -

Pflicht, den Wünschen des Betreuten zu entsprechen; \S 1908i Abs. 1, 1839 - Auskunftspflicht;

SS $1908 \mathrm{i}$ Abs. 1, 1840 - Bericht und Rechnungslegung.

${ }^{890}$ D.h. kein mit dem Betreuer vergleichbares Amt (Rieger FS Schwab S. 1043, 1054).

${ }^{891}$ Diesen Vorschlag macht Lipp FS Bienwald S. 177, 185; ihm folgt Zimmermann Rn. 371. 
zeitigen Fassung und ermöglicht durch eine doppelte Vorkehrung auf Seiten des Betroffenen die Wahrung seiner Wünsche. Die Beachtung des Betroffenenwillens im Betreuungsrecht müsste diese Möglichkeit aber bereits durch die Nennung des Wunschbetreuers in der Betreuungsverfügung gewähren. Daher ist die Position des Vorgeschlagenen als Recht i.S.d. \59 FamFG anzunehmen: Der Wunschbetreuer erwirbt mit seiner Nennung in der Betreuungsverfügung nicht nur eine Pflicht zur Übernahme der Betreuung nach SS 1897 Abs. 4, 1989 Abs. 1, sondern auch ein eigenes Recht, aus dem er nach $\int 59$ FamFG bei unrechtmäßiger Missachtung des Betroffenenvorschlags aus subjektiver Rechtsverletzung beschwerdeberechtigt ist. ${ }^{892}$ Dies Auslegung entspricht dem Beschwerderecht des Bevollmächtigten aus $\int 59$ FamFG und dem Vorschlagsrecht der Eltern nach $\iint 1776$, 1779 .

\section{Einrichtung der Betreung auf Antrag des Betroffenen}

\69g Abs. 1 FGG behandelte nur das Beschwerderecht gegen die Bestellung eines Betreuers von Amts wegen, nicht gegen die Einrichtung der Betreuung auf Antrag des Betroffenen. Dann ergab sich ein Recht zur Beschwerde ausschließlich aus \20 FGG/ \59 FamFG.

Nach \303 FamFG hat nach Abs. 1 die zuständige Behörde ein Beschwerderecht, ${ }^{893}$ nach Abs. 3 der Verfahrenspfleger und gem. Abs. 4 der Betreuer, soweit die gerichtliche Entscheidung seinen Aufgabenkreis betrifft. Das Beschwerderecht nach Abs. 2 besteht hingegen nur gegen von Amts wegen ergangene Entscheidungen. Also auch für die Fälle, in denen die Einrichtung der Betreuung von Amts wegen erfolgte. Wurde die Betreuung auf einen Antrag des Betroffenen hin gem. \1896 Abs. 1 S. 1 1. Alt. eingerichtet beschränkt sich der Kreis der Beschwerdeberechtigten nach \303 FamFG auf die zuständige Behörde, den Verfahrenspfleger und den in seinen Aufgabenkreis betroffenen Betreuer. Darüber hinaus ist nach der allgemeinen Regel $\int 59$ FamFG derjenige beschwerdeberechtigt, dessen Rechte durch den Beschluss beeinträchtigt wurden.

Fraglich ist, ob der nicht zum Betreuer ernannte Wunschbetreuer, wie bei der Einrichtung der Betreuung von Amts wegen, ebenfalls nach \59 FamFG ein Beschwerderecht hat. Bei der Einrichtung der Betreuung auf Antrag des Betroffenen und der Einrichtung von Amts wegen geht es ausschließlich um die Rechte und Interessen des Betroffenen, ${ }^{894}$ speziell um die Einhaltung seines in $\int 1897$ Abs. 4 normierten Vorschlagsrechts. Die Begrenzung der Beschwerdeberechtigten wird

\footnotetext{
892 Rieger FS Schwab S. 1043, 1053; anderer Ansicht sind: Zimmermann FamRZ 1992, 342; BayObLG FamRZ 1992, 341; BayObLG FamRZ 2003, 1219, 1220: Sie lassen kein Recht und keine gesicherte Anwartschaft zu, aber eine Aussicht auf ein derartiges Recht.

${ }^{893}$ Nach der Gesetzesbegründung soll sich dieses Recht auf die Verfahren beschränken, in denen die Behörde bereits in erster Instanz zu beteiligen war, vgl. BT-Drucks. 16/6308 S. 271. Diese Einschränkung findet in $₫ 303$ Abs. 1 FamFG jedoch keine Beachtung.

894 Rieger FS Schwab S. 1043, 1051 ff.
} 
aufgrund der sich daraus ergebenden Inkonsequenz innerhalb des Gesetzes kritisiert. ${ }^{895}$ Andere sehen sie als rechtpolitisch bedenklich an, weil gem. \1896 Abs. 2 S. 2 auch ein Geschäftsunfähiger einen Antrag auf Einrichtung seiner Betreuung stellen kann. 896

Das Gesetz grenzt den Kreis der Beschwerdeberechtigten ein, weil der Betroffene in der Lage war, für sich einen eigenen Antrag zu formulieren. ${ }^{897}$ Der Betroffene müsste dann auch in der Lage sein, seinen Wunschbetreuer zu benennen. 898 Diese Schlussfolgerung ist konsequent. Denn die Hilfsbedürftigkeit des Betroffenen, der einen Antrag stellen kann, ist noch nicht derart ausgewachsen, dass er desselben Schutzes bedarf, wie derjenige, für den aufgrund fehlender Eigenständigkeit eine Betreuung von Amts wegen eingerichtet wird. Dabei ist auch davon auszugehen, dass der Betroffene zumindest im Verfahren der Betreuerbestellung seine Rechte noch umfassend selber wahrnehmen kann. In diesem Fall bedarf es keines Beschwerderechts des Wunschbetreuers. Es ist davon auszugehen, dass der Betroffene nicht nur seinen aktuellen Wunsch äußern bzw. auf Wünsche in der Betreuungsverfügung verweisen kann, sondern dass er darüber hinaus auch noch selber in der Lage ist, diese Wünsche gem. \59 FamFG durchzusetzen. Ein Beschwerderecht des Wunschbetreuers bedarf es daher in diesen Fällen nicht.

\section{B. Durch den Betreuer}

I. Missachtung der Wunschbefolgungspflicht

Soll das Handeln des Betreuers bei der Führung der Betreuung beurteilt werden, ist zwischen Innen- und Außenverhältnis zu differenzieren. Im Innenverhältnis zwischen Betreutem und Betreuer ist der Betreuer an den Betroffenenwunsch gebunden. Übergeht er diesen ungerechtfertigt, stellt dies eine Pflichtwidrigkeit des Betreuers dar. Übergeht er einen Wunsch des Betroffenen, der die gesetzlich zwingenden Grenzen missachtet, handelt er rechtmäßig. Ein solcher Wunsch des

\footnotetext{
895 Staudinger/Bienwald \1896 Rn. 66; v.a. mit Blick auf das den Angehörigen in \1897 Abs. 4 und 5 entgegengebrachte Vertrauen, aber auch dann, wenn der Betroffene zwar den Antrag stellt, das Gericht einen Betreuer dann aber für mehr als nur die beantragten Aufgabenkreise bestellt.

${ }^{896}$ MünchKommBGB/Schwab \$1896 Rn. 122.

${ }^{897}$ Das Beschwerderecht der Betreuungsbehörde wurde gem. \303 Abs. 1 FamFG auch für die Fälle normiert, in denen der Betroffene einen Antrag auf Einrichtung der Betreuung gestellt hat. Damit steht ihr ein Beschwerderecht auch gegen den Willen des Betroffenen zu. Damit sollen kostenintensive Betreuungsverfahren eingedämmt werden, in denen der Betroffene zur Regelung seiner Angelegenheiten entgegen seines eigenen Antrags tatsächlich in der Lage ist (BT-Drucks. 16/6308 S. 271).

898 Die Anforderungen an den Antrag des Betroffenen müssen den Anforderungen an den Wunsch des Betroffenen im Betreuungsverfahren und in der Betreuung gleich gesetzt werden vgl. dazu \2 C. sowie Lipp S. 78 f. Die herrschende Meinung fordert hier zu hohe inhaltliche Anforderungen, vgl. nur Staudinger/Bienwald \$ 1896 Rn. 58 ff.
} 
Betreuten ist nicht verbindlich. Missachtet er die Wünsche des Betroffenen, weil ihr Befolgen eine Wohlgefährdung bedeutete oder die Umsetzung ihm unzumutbar ist, handelt er ebenfalls rechtmäßig, vgl. \ 1901 Abs. 3 S. 1. Bestimmt er seine Pflichten durch falsche Gesetzesauslegung oder -anwendung nicht korrekt und setzt sie falsch um, handelt er rechtswidrig.

Im Außenverhältnis, d.h. im Verhältnis zu Dritten vertritt der Betreuer den Betroffenen nach den Grundsätzen des Vertretungsrechts, vgl. \ 1902 i.V.m. $\iint 164$ ff. Die Bindung des Betreuers an die Wünsche des Betreuten schränkt sein Handeln im Außenverhältnis grundsätzlich nicht ein. ${ }^{899}$

\section{Rechtsfolgen}

Die Rechtsfolgen sind nach Innen- und Außenverhältnis zu unterscheiden. Missbraucht der Betreuer seine gesetzliche Vertretungsmacht, die er im Außenverhältnis wahrnimmt, gelten die allgemeinen Regelungen des Vertretungsrechts gem.

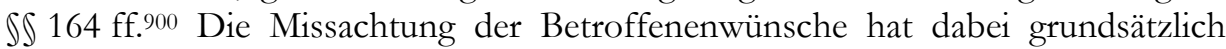
keine Auswirkung. Ausnahmsweise entfällt seine Vertretungsmacht aus \1902, soweit er diese missbraucht. ${ }^{901}$ Dies ist dann gegeben, wenn der Betreuer seine Vertretungsmacht pflichtwidrig missachtet oder wenn dies dem Dritten bekannt oder evident ist. ${ }^{902}$

Im Innenverhältnis, also zwischen Betreutem und Betreuer haben die Pflichtverletzung oder die fehlenden gesetzlichen Voraussetzungen, die an den Betreuer zu stellen sind, andere Folgen. Eine Pflichtwidrigkeit bei der Führung der Betreuung zieht aufsichtsrechtliche Maßnahmen des Betreuungsgerichts gem. SS $1908 \mathrm{i}$ Abs. 1 S. 1 i.V.m. 1837 Abs. 2, 3 nach sich. ${ }^{903}$ Trägt der Betreuer Wünschen des Betreuten nicht Rechnung, kann die Verletzung der Wunschbefolgungspflicht nach \ 1901 Abs. 2 und $3^{904}$ und damit die Missachtung der Betreuungsverfügung gerügt werden. ${ }^{905}$ Entsteht aus dem unbegründeten Übergehen des Vorschlags ein Schaden, so haftet dafür der Betreuer gem. JS 1833 i.V.m. 1908i Abs. 1 S. $1 .{ }^{906}$ Gravierende und wiederholte Missachtung der Betroffenenwünsche begründen Zweifel an seiner Eignung und können zu seiner Entlassung führen. ${ }^{907}$ Auf sein

\footnotetext{
${ }^{899}$ MünchKommBGB/Schwab \$ 1901 Rn. 20; LG Berlin FamRZ 2000, 1526, 1529; Schwab FamRZ 1990, 681, 683; Erman/Holzhauer \1901 Rn. 25; Soergel/Zimmermann \$ 1901 Rn. 9; HKBUR/Bauer \1901 Rn. 56; Knittel \$ 1901 Rn. 14; Motive zum BGB Band 4, S. 1086.

900 MünchKommBGB/Schwab \$ 1902 Rn. 16.

901 HK-BUR/Bauer \$1901 Rn. 57; MünchKommBGB/Schwab \1902 Rn. 16.

902 Heute herrschende Meinung, vgl. nur Lipp Vorsorgeverfügungen \18 Rn. 91.

903 MünchKommBGB/Schwab \$1901 Rn. 19.

904 MünchKommBGB/Schwab \ 1901 Rn. 19.

905 Lipp Vorsorgeverfügungen \18 Rn. 51; MünchKommBGB/Schwab \1908b Rn. 5, Erman/-

Holzhauer \1908b Rn. 6; Jürgens/Mertens \$1908b Rn. 2; BayObLG vom 28.07.1997 Az.: 3Z BR

147/97; Befolgen der Wünsche als Rechtspflicht, vgl. Perau MittRhNotK 1996, 285, 289.

906 MünchKommBGB/Schwab \1901 Rn. 19.

${ }^{907}$ Lipp Vorsorgeverfügungen $\$ 18$ Rn. 87.
} 
Verschulden kommt es dabei nicht an, da die Maßnahme allein dem Schutz des Betroffenenwohls dient. ${ }^{908}$ Das Gericht wird dann von Amts wegen tätig, weshalb die Anregung dieser Maßnahme von dem Betreuten und jedem Dritten erfolgen kann. Liegt eine schuldhafte Verletzung der Pflicht aus $\ 1901$ Abs. 3 vor, macht sich der Betreuer gem. IS 1901 i Abs. 1 S. 1, 1833 schadensersatzpflichtig und u.U. strafbar. ${ }^{909}$

Nach $\int 1908$ b Abs. 1 hat das Betreuungsgericht den Betreuer zu entlassen, wenn seine Eignung, die Angelegenheiten des Betreuten zu besorgen, nicht mehr gewährleistet ist oder ein anderer wichtiger Grund zur Entlassung vorliegt. Mangelnde Eignung des Wunschbetreuers macht seine Benennung in der Betreuungsverfügung daher wirkungslos. ${ }^{910}$ Das Vorliegen der Eignung unterliegt der vollen Nachprüfbarkeit in der Rechtsbeschwerde. ${ }^{911}$ Wurde eine ungeeignete Person erst einmal wirksam zum Betreuer bestellt, ist sie gem. $₫ 1908$ b Abs. 1 S. 1 vom Betreuungsgericht zu entlassen. Der daraus entstehende Schaden ist ggf. gem. \839 und Art. 34 GG zu ersetzen. ${ }^{912}$ Wird der Betreuer trotz bestehender Ausschlussgründe gem. $\ 1897$ Abs. 3 bestellt, ist er gem. $\ 1908 \mathrm{~b}$ Abs. 1 als ungeeignet $^{913} \mathrm{zu}$ entlassen.

Erfolgt eine Bestellung des Betreuers trotz Unzumutbarkeit, hat dies erst Auswirkungen, wenn der Betreuer gem. $\iint 1898$ Abs. 1, 1908b Abs. 2 seine Entlassung beantragt. \1908b Abs. 2 regelt die Situation, in der die Entlassungsgründe erst nach seiner Bestellung eintreten. Aufgrund der Kontinuität in der Führung der Betreuung ist hier ein höherer Maßstab an die Zumutbarkeit als bei $\$ 1898$ anzusetzen. ${ }^{914}$ Aufgrund der besonderen Beachtung des Vertrauensverhältnisses zwischen Betreuer und Betreutem ist eine entsprechende Anwendung jedoch zu befürworten.

Durch die Übernahmeerklärung des Betreuers nach $\int 1898$ Abs. 2 soll sich dieser im konkreten Fall zur Betreuung vor allen Beteiligten erklären. ${ }^{915}$ Aufgrund der bestehenden Pflicht zur Übernahme ist die Erklärung als verpflichtender Dia$\log$ zum Zwecke des Informationsaustauschs in Vorbereitung der Erfüllung der

\footnotetext{
908 MünchKommBGB/Schwab \1908b Rn. 5.

909 Pflichtverletzungen aus \1901 Abs. 3 können z.B. den Straftatbestand der Untreue gem. \266

Strafgesetzbuch oder der Körperverletzung gem. \223 Strafgesetzbuch wegen einer Behandlung gegen den Willen des Betreuten erfüllen.

910 Epple BWNotZ 1992, 27, 28.

911 Staudinger/Bienwald \1897 Rn. 12; Bienwald/Sonnenfeld/Hoffmann \1897 Rn. 73; BayObLG FamRZ 1996, 509, 510; BayObLG FamRZ 2001, 1249; BayObLG FamRZ 2002, 768, 769. Bei Nichtgeeignetheit des Betroffenen soll keine sofortige Beschwerde möglich sein (BT-Drucks. $11 / 4528$ S. 129).

912 Bienwald/Sonnenfeld/Hoffmann \1897 Rn. 75.

913 HK-BUR/Bauer $\$ 1897$ Rn. 50; BayObLG BtPrax 1997, 36; LG Berlin BtPrax 1997, 39; LG

Stuttgart BtPrax 1996, 75. Nach Bienwald muss er dann „aus wichtigem Grund" entlassen werden, vgl. FF 2003, 202, 203 und 205.

${ }^{914}$ MünchKommBGB/Schwab\$1908b Rn. 16.

915 Vgl. dazu Ausführungen unter $\$ 3$ B. V.
} 
Bürgerpflicht anzusehen. Die Ernennung des Betreuers trotz fehlender Übernahmeerklärung ist rechtmäßig. Ihr Fehlen könnte als zwingende gesetzliche Voraussetzung der Betreuungsbestellung evtl. eine rechtmäßige Entscheidung herbeiführen, jedoch zur Aufhebbarkeit der Betreuung gem. S 1908b Abs. 1 S. 1 aus wichtigem Grund führen. ${ }^{916}$ Die Entlassung des Betreuers nach $\int 1908 \mathrm{~b}$ erfolgt zum Schutz des Betreuten. ${ }^{917}$ Die fehlende Übernahmeerklärung ist kein vergleichbarer Grund und kann daher nicht zur Entlassung des Betreuers führen.

Bei Missachtung des Grundsatzes der ehrenamtlichen vor der Berufsbetreuung hat das Betreuungsgericht den Betreuer gem. SS 1897 Abs. 6, 1908b Abs. 1 S. 1 aus wichtigem Grund zu entlassen. ${ }^{918}$ Verkennt das Gericht bei der Bestellung des Betreuers den Vorrang der privaten, auch berufsmäßigen Einzelbetreuung, hat es statt den Vereins- oder Behördenbetreuer den Einzelbetreuer gem. SS 1897 Abs. 1, 1900, 1908b Abs. 5 zu ernennen. ${ }^{919}$ Mit Ernennung erfolgt die Entlassung des vorherigen Betreuers.

Das Missachten der Betreuungsbedürftigkeit auf Seiten des Betroffenen, Vorliegen der Geschäftsunfähigkeit oder beschränkten Geschäftsfähigkeit ${ }^{920}$ auf Seiten des Betreuers führt zu seiner mangelnden Eignung im weiten Sinn, ${ }^{921}$ so dass er zwar wirksam zum Betreuer bestellt werden konnte, aber nach $\int 1908$ b Abs. 1 S. 1 eine Entlassung nötig ist. Ein wichtiger Grund für die Entlassung liegt bei Interessenkollisionen in Vermögensfragen ${ }^{922}$ vor. Gleiches gilt nach \1908b Abs. 1 S. 2 bei vorsätzlich falscher Abrechnung durch den Betreuer.

\section{Ergebnis zu $\$ 6$}

Die Missachtung des zulässigen Betroffenenvorschlags bewirkt nicht die Nichtigkeit der gerichtlichen Entscheidung. Diese ist wirksam, jedoch mittels der Beschwerde gem. IS 303 ff. FamFG angreifbar. Ist der Wunschbetreuer keine in \303 FamFG genannte Person, ergibt sich seine Beschwerdeberechtigung aus subjektiver Rechtsverletzung gem. \59 FamFG. Das subjektive Recht erwächst aus seiner Nennung in der Betreuungsverfügung als Wunschbetreuer und entsteht mit Einleitung eines Verfahrens. Dies gilt jedoch nur für die Einrichtung der Betreuung von Amts wegen, weil der Betroffene dann besonders schutzwürdig ist. Bei der Einrichtung der Betreuung auf Antrag des Betroffenen kann nur er die Auswahl des Betreuers nach \59 FamFG rügen.

\footnotetext{
916 Zumindest bei der Bestellung eines Vereins-/ Behördenbetreuers ohne Vorliegen seiner wirksamen Einwilligung (Bienwald/Sonnenfeld/Hoffmann \ 1897 Rn. 72).

917 Bienwald/Sonnenfeld/Hoffmann $\int 1908$ b Rn. 8.

918 Bienwald FF 2003, 202, 205.

919 Bienwald FF 2003, 202, 205; Palandt/Diederichsen \ 1900 Rn. 1.

920 Bienwald/Sonnenfeld/Hoffmann \ 1897 Rn. 44; Staudinger/Engler \ 1780 Rn. 6.

$921 \mathrm{Vgl}$. BT-Drucks. 11/4528 S. 125.

922 Nach BayObLG bedarf es der konkreten Gefahr (BayObLG FamRZ 1993, 988).
} 
Missachtet der Betreuer die Vorgaben der Betreuungsverfügung, haftet er im Außenverhältnis nach den Grundsätzen des Stellvertretungsrechts. Gegenüber dem Betreuten stellt das Übergehen seines Wunsches eine Pflichtverletzung dar, die aufsichtsrechtliche Maßnahme nach sich zieht. 


\section{\ 7 Ergebnis und Ausblick}

Die vorliegende Untersuchung zeigt, dass der Einzelne eine mögliche eigene zukünftige Betreuung umfassend selbstbestimmt ausgestalten kann. Dies gilt sowohl für das Verfahren der Betreuerbestellung 923 als auch für die Führung der Betreuung $^{924}$. Es ist möglich durch antizipierte Äußerung von verbindlichen Wünschen und Vorstellungen, welche im Rahmen einer Betreuungsverfügung festgehalten werden. ${ }^{925}$ Die einfachgesetzliche Anerkennung dieser Wünsche und Vorstellungen des Betroffenen im Betreuungsrecht ist Ausfluss des grundrechtlich garantierten Selbstbestimmungsrechts des Einzelnen, ${ }^{926}$ ihre weitmöglichste Beachtung wesentliches Ziel des Bereuungsrechts. ${ }^{927}$

Zwingend beachtlich, und somit durch den Betroffenen mittels individueller Wünsche unabdingbar, sind bestimmte gesetzliche Vorgaben: Der von ihm benannte Wunschbetreuer muss zur Übernahme der Einzelbetreuung geeignet ${ }^{928}$ und bereit ${ }^{929}$ sein und darf in keiner in $\$ 1897$ Abs. 3 genannten Verbindung zur

\footnotetext{
923 Vgl. \ 4.

${ }^{224}$ Vgl. $\int 5$.

925 Vgl. \$ 2 C. II. 2.

926 Vgl. \$ 2 B. II.

927 Vgl. $\int 2$ B. III.

928 Vgl. \ 3 B. I.

${ }_{929}$ Vgl. \ 3 B. V.
} 
Einrichtung oder dem Heim stehen, in dem der Betroffenen lebt ${ }^{930}$. Zudem muss er geschäftsfähig ${ }^{931}$ sein, darf im einschlägigen Aufgabenkreis nicht selbst unter Betreuung stehen ${ }^{932}$ und die Übernahme der Betreuung muss für ihn zumutbar ${ }^{933}$ sein. Die Verfahrenspflegschaft ist nur durch alternative, d.h. privatautonome Beauftragung eines Verfahrensvertreters vermeidbar.934 Die Person des Verfahrenspflegers kann durch den Betroffenen ausgewählt, bestimmte Personen von diesem Amt durch ihn ausgeschlossen werden. ${ }^{935}$ Ist der Betroffene mittellos, ist die gesetzlich bestimmte Vergütung des Verfahrenspflegers undisponibel. ${ }^{936}$ Auch die Anhörung des Betroffenen ist zwingend vorzunehmen, 937 der Betroffene kann lediglich ihren Ort vorschlagen ${ }^{938}$. Vom Sachverständigengutachten kann nicht aufgrund des Betroffenenwunsches abgesehen werden, ${ }^{939}$ aber die Person des Gutachters von ihm verbindlich ausgewählt und bestimmte Personen als Gutachter ausgeschlossen werden ${ }^{940}$. Die Regelungen zur gerichtlichen Zuständigkeit und einstweiligen Betreuerbestellung entziehen sich ebenfalls der individuellen Einflussnahme. ${ }^{941}$

Wünsche über die Vornahme oder Nichtvornahme medizinischer Behandlungen sind gem. \$S 1901a, 1901b verbindlich. ${ }^{942}$ Die vom Gesetz in \$S $1904-1908$ vorgeschriebenen Genehmigungsvorbehalte sind zwingend. Eine Sterilisation nach \ 1905 kann durch antizipierten Entzug der Einwilligung mittels der Betreuungsverfügung verhindert werden, ${ }^{943}$ eine Unterbringung nach $\int 1906$ hingegen nicht. Abs. 1 S. 1 bestimmt jedoch, dass sie nur zulässig ist, solange sie nach \ 1901 Abs. 3 zum Wohl des Betreuten erforderlich ist. ${ }^{944}$ Gleiches gilt für die in $\int 1907$ regulierte Aufgabe seiner Wohnung ${ }^{945}$ und seiner Ausstattung ${ }^{946}$ gem. \ 1908. In der Verwaltung seines Vermögens wird der Betroffene durch \1908i Abs. 2 i.V.m. \1804 eingeschränkt. V.a. die Regelung zum geltenden Schenkungsverbot ist jedoch teleologisch zu reduzieren. ${ }^{947}$

\footnotetext{
930 Vgl. \ 3 B. III.

931 Vgl. $\int 3$ B. VI.

932 Vgl. $\int 3$ B. VII.

933 Vgl. 5 B. V.

934 Vgl. 4 B. II.

935 Vgl. $\int 4$ B. I.

936 Vgl. \ 4 B. III.

937 Vgl. 54 C. I.

938 Vgl. 4 C. II.

${ }^{939}$ Vgl. $\int 4$ D. I.

940 Vgl. $\int 4$ D. II.

941 Vgl. $\int 4$ E. und F.

942 Vgl. 5 A. II.

943 Vgl. $\int 5$ A. III. 1.

944 Vgl. $\int 5$ A. III. 2.

945 Vgl. $\int 5$ A. III. 3. b).

946 Vgl. ( 5 A. III. 3. c) aa).

947 Vgl. \ 5 A. III. 3. c) bb)
} 
Die Missachtung des zulässigen Betroffenenvorschlags hinsichtlich des Wunschbetreuers durch das Gericht macht die Beschwerde nach

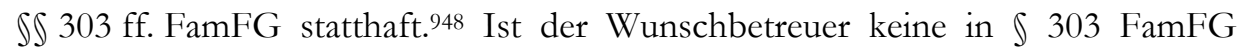
genannte Person, ergibt sich seine Beschwerdeberechtigung für die Einrichtung der Betreuung von Amts wegen aus subjektivem Recht gem. \59 FamFG durch die Nennung in der Betreuungsverfügung. ${ }^{949}$ Bei der Einrichtung der Betreuung auf Antrag des Betroffenen kann nur der Betroffene die Auswahl des Betreuers nach $₫ 59$ FamFG rügen. ${ }^{950}$ Missachtet der Betreuer die Wünsche des Betroffenen, hat dies im Außenverhältnis grundsätzlich keine Auswirkung, stellt gegenüber dem Betroffenen jedoch eine Pflichtverletzung dar, die aufsichtsrechtliche Maßnahmen des Gerichts zur Folge hat und ggf. zu seiner Entlassung führen kann. ${ }^{951}$

Alle übrigen Fragen des Lebens kann der Betroffene im Rahmen der allgemeinen gesetzlichen Grenzen nach seinen Wünschen und Vorstellungen in der Betreuungsverfügung regeln und für sich beantworten. ${ }^{552}$ An diese sind Betreuungsgericht und Betreuer gebunden. ${ }^{953}$ Verbindlich sind sie dann, wenn sie ernsthaft, bewusst und frei von Irrtum und Einfluss Dritter gefasst wurden. ${ }^{954}$ Ihre Verbindlichkeit entfällt nur, wenn die Wünsche aufgrund eines Willensmangels im Zustand fehlende Eigenverantwortung gefasst wurden, bzw. der Betroffene sich durch die Umsetzung eines solchen Wunsches selbst schädigen würde. ${ }^{955}$ Diese Anforderungen sind an die Wünsche der Betreuungsverfügung zu stellen. Die Betreuungsverfügung unterliegt keinen formalen Erfordernissen. 956

Dieses Ergebnis zeigt, dass das Gesetz dem Betroffenen mittels der Betreuungsverfügung einen vergleichsweise großen Einfluss auf die Modalitäten der Einrichtung und Ausgestaltung seiner eigenen Betreuung gewährt. Es begrenzt diesen nur in den wenigen Bereichen, in denen es zu seinem Schutz allgemein erforderlich erscheint. Die Genehmigungsvorbehalte der $\$ \$ 1904$ - 1908 entziehen beispielsweise die inhaltliche Entscheidung nicht dem Einfluss des Betroffenen, weil sie auf den allgemeinen Entscheidungsmaßstab in $\$ 1901$ Abs. 2 und 3 verweisen, den der Betroffenen selber definiert. 957 Sie fordern vielmehr, aufgrund ihres weitreichenden Eingriffs in die Rechte des Betroffenen, die Genehmigung des Gerichts. Dieses prüft nicht den Inhalt der Entscheidung, sondern das Vorliegen der Voraussetzungen und garantiert somit die Beachtung der individuellen Entscheidungen des Betroffenen.

\footnotetext{
$948 \mathrm{Vgl.} \$ 6$ A. II.

${ }^{949}$ Vgl. $\$ 6$ A. II. 1.

950 Vgl. $\int 6$ A. II. 2.

951 Vgl. \$ 6 B. II.

952 Vgl. $\$ 2$ C. III.

953 Vgl. \$ 2 C. II.

954 Vgl. § 2 C. II. 2. c.

955 Vgl. \$ 2 C. II. 3.

956 Vgl. \$ 2 C. IV.

957 Vgl. $\int 5$ A. III.
} 
Die Betreuungsverfügung bietet daher nicht nur eine Möglichkeit zur antizipierten Ausübung des Selbstbestimmungsrechts in der Betreuung, sondern garantiert diese. Damit schließt sie die Gefahr der Fremdbestimmung aus.

Im Vergleich zur Vorsorgevollmacht bietet die Betreuungsverfügung somit ein ungleich höheres Maß, Selbstbestimmung umzusetzen, wenn ihre tatsächliche Ausübung nicht mehr möglich ist. Gegenüber der Patientenverfügung ermöglicht die Betreuungsverfügung nicht nur die Regelung von Fragen der Gesundheit, sondern aller Lebensbereichen.

Insgesamt ist festzustellen, dass bislang die Möglichkeiten der Betreuungsverfügung in fast allen Fällen unterschätzt wurden. Die Einordnung der Betreuungsverfügung lediglich als Instrument zur Benennung des Wunschbetreuers wird ihren Regelungsmöglichkeiten nicht gerecht. Vielmehr ist sie als Instrument zu bewerten, dass die Ausübung des Selbstbestimmungsrechts in der Betreuung umfassend gewährt und garantiert. Mit Blick auf den demographischen Wandel und der damit verbundenen steigenden Zahl der Betreuungen wird die Betreuungsverfügung in Zukunft einen ebenso zahlreichen wie vielfältigen Anwendungsbereich finden. 


\section{Literaturverzeichnis}

Abrens, Martin

Autonomie in Fesseln - Vorsorgevollmacht und Vorsorgeverhältnis an den Schranken des Rechtsberatungsgesetzes, BtPrax 2005, 163 ff.

Alperstedt, Ralf

Dauerergänzungsbetreuung bei tatsächlicher Verhinderung?,

BtPrax 2001, 106 ff.

Bamberger, Heinz Georg/ Roth, Herbert

Kommentar zum Bürgerlichen Gesetzbuch. Band 3 SS 1297 - 2385, EGBGB, CISG, 2. Auflage, München 2008

Zitiert: Bamberger/Roth/Bearbeiter

Barth, Peter

Das Sachwalterrechts-Änderungsgesetz 2006. Die Reform im Überblick, iFamZ 2006, $138 f f$.

Barth, Peter/ Ganner, Michael (Hrsg.)

Handbuch des Sachwalterrechts. Mit Angehörigenvertretung,

Vorsorgevollmacht und Patientenverfügung, Wien 2007

Bartolomeyczik, Horst

Die subjektiven Merkmale der Willensbildung, in: Ferid, Murad (Hrsg.),

Festschrift für Hans G. Ficker zum 70. Geburtstag am 20. Juli 1967,

Frankfurt, Berlin 1967, S. 51 ff. 
Bassenge, Peter/ Roth, Herbert

Gesetz über die Angelegenheiten der freiwilligen Gerichtsbarkeit:

Rechtspflegergesetz, Kommentar, 11. Auflage, Heidelberg 2007

Bauer, Axel/ Klie, Thomas

Patientenverfügungen/Vorsorgevollmachten - richtig beraten? 2. Auflage, Heidelberg 2005

Bauer, Axel/ Klie, Thomas (Hrsg.)

Heidelberger Kommentar zum Betreuungs- und Unterbringungsrecht, Stand: 70. Aktualisierung, September 2009

Zitiert: HK-BUR/Bearbeiter

Bauer, Axel/ Rink, Jürgen

Kritik des Entwurfs eines Gesetzes zur Änderung des Betreuungsrechts sowie weitere Vorschriften (Betreuungsrechtsänderungsgesetz - BtÄndG, Stand: 7. Februar 1996) 2. Teil, BtPrax 1996, 158 ff.

Baumann, Wolfgang/ Hartmann, Christian

Die zivilrechtliche Absicherung der Patientenautonomie am Ende des Lebens aus Sicht der notariellen Praxis, DNotZ 2000, $594 \mathrm{ff}$.

Becker, H./ Mettheis, R./ Hennies, G./ Schirop, T.

Behandlungsabbruch - Patientenverfügung (Patiententestament), Betreuungsverfügung und Vorsorgevollmacht. Empfehlung der Ethikkommission der Ärztekammer Berlin, Intensivmed 1999, 71 ff.

Berger, Christian

Privatrechtliche Gestaltungsmöglichkeiten zur Sicherung der Patientenautonomie am Ende des Lebens, JZ 2000, $797 \mathrm{ff}$.

Bienwald, Werner

Die Vorsorgevollmacht - ein gleichwertiger Ersatz der Betreuerbestel-

Bienwald, Werner lung?, BtPrax 1998, $164 \mathrm{ff}$.

Der Betreuer mit dem Aufgabenkreis nach $\int 1896$ Abs. 3 BGB, Rpfleger 1998, $231 \mathrm{ff}$.

Bienwald, Werner

Weitere Unvollkommenheit der Vorsorgevollmacht gegenüber der Betreuung,

BtPrax 1999, 92 ff.

Bienwald, Werner

Geltendmachung von Aufwand und Vergütung. Eine Behörde oder ihre Mitarbeiter werden zum ,Pfleger für das Verfahren', Rpfleger 1999, 429 ff. Bienwald, Werner

Betreuungsrecht, Gesetz zur Reform des Rechts der Vormundschaft und Pflegschaft für Volljährige (BtG) und Gesetz über die Wahrnehmung behördlicher Aufgaben bei der Betreuung Volljähriger (BtBG) Kommentar, 3. Auflage, Bielefeld 1999

Zitiert: Bienwald BtG, 3.Aufl. 


\section{Bienwald, Werner}

Anmerkung zu BVerfG vom 07.06.2000, FamRZ 2000, 1280

Bienwald, Werner

$\mathrm{Zu}$ den Anforderungen an die Qualifikation des Betreuers, FamRZ 2000, 1314 ff.

Bienwald, Werner

Vorsorgeverfügungen und ihre Bedeutung für das Vormundschaftsgericht, BtPrax 2002, 227 ff.

Bienwald, Werner

Verfahrenspflegschaftsrecht. Ein Handbuch. Bielefeld 2002

Zitiert: Bienwald Verfahrenspflegschaftsrecht

Bienwald, Werner

Wie wird man einen Betreuer und ähnliche Interessensvertreter wieder los?, FF 2003, 202 ff.

Bienwald, Werner

Anmerkung zu LG Kassel, FamRZ 2006, 1302

Bienwald, Werner/ Sonnenfeld, Susanne/ Hoffmann, Birgit

Betreuungsrecht Kommentar, 4. Auflage, Bielefeld 2005

Zitiert: Bienwald/ Sonnenfeld/ Hoffmann

Binschus, Wolfgang

Zur Vorsorgevollmacht, Der Amtsvormund 1998, 275 ff.

Birmanns, Martin

Muster einer Betreuungsverfügung, NWB Nr. 52/53 vom 21.12.1998, S. $4355 \mathrm{f}$.

Bittler, Jan

Patientenverfügung und andere Vorsorgemöglichkeiten. So entscheiden

Sie über Ihr Leben autonom, 7. Auflage, Regensburg, Berlin 2007

Bobenhausen, Dieter

Wohnungskündigung durch den Betreuer, Rpfleger 1994, 13 ff.

Bobenhausen, Dieter

Konkurrenz zwischen dem Willen des Betreuten und des Betreuers:

Gesetzliche Vertretung - Kontosperre - Schenkung, BtPrax 1994, 158 ff.

Bork, Reinhard

Sind SS 50, 67 FGG verfassungskonform?, FamRZ 2002, 67 ff.

Borowski, Martin

Intendiertes Ermessen, DVBl. 2000, 149 ff.

Brox, Hans / Walker, Wolf-Dietrich

Erbrecht, 22. Auflage Köln 2007

Brucker, Uwe

Selbstbestimmt Vorsorge treffen. Teil I: Vorsorgevollmacht und Betreuungsverfügung, Die BKK 2002, 198 ff. 
Bübler, Martin

Vorsorgevollmacht zur Vermeidung einer Gebrechlichkeitspflegschaft oder Betreuung, BWNotZ 1990, 1 ff.

Bübler, Ernst/ Kren, Rita/ Stolz, Konrad

Betreuungsrecht und Patientenverfügung im ärztlichen Alltag, 2. Auflage, München 2006

Bumiller, Ursula/ Winkler, Karl

Freiwillige Gerichtsbarkeit: Gesetz über die Angelegenheiten der freiwilligen Gerichtsbarkeit, 8. Auflage, München 2006

Bumiller, Ursula/ Harders, Dirk

Gesetz über das Verfahren in Familiensachen und in den Angelegenheiten

Bund, Uwe der freiwilligen Gerichtsbarkeit (FamFG), 9. Auflage, München 2009

Die Notarkosten bei Vorsorgevollmacht mit Betreuungs- und Patienten-

Bund, Uwe verfügung, RNotZ 2004, 23 ff.

Erneut: Die Beurkundungsgebühren der General- und Vorsorgevollmacht mit Betreuungs- und Patientenverfügung Teil I, JurBüro 2005, 622 ff.

Bundesärztekammer

Grundsätze der Bundesärztekammer zur ärztlichen Sterbebegleitung vom 11.09.1998, NJW 1998, $3406 \mathrm{f}$.

Bundesärztekammer

Handreichungen für Ärzte zum Umgang mit Patientenverfügungen, DÄB1. 96, Heft 43, 29. Oktober 1999 A-2720 (23 f.)

Bundesministerium der Justiz. (Hrsg.)

Diskussions-Teilentwurf Gesetz über die Betreuung Volljähriger (Betreuungsgesetz - BtG) November 1987, Köln 1987

Zitiert: BtG-DiskE

Bürgle, Helmut

Auf dem Weg zu einem neuen Betreuungsrecht, NJW 1988, 1881 ff.

Bydlinski, Franz

Juristische Methodenlehre und Rechtsbegriffe, 2. Auflage, Wien 1991

Zitiert: Bydlinski

Canaris, Claus-Wilhelm

Verstöße gegen das verfassungsrechtliche Übermaßverbot im Recht der

Geschäftsfähigkeit und im Schadensersatzrecht, JZ 1987, 993 ff.

Chauvistré, Ralph

Vorsorgevollmacht und rechtliche Betreuung: Betreuungsverfügung und

Patientenverfügung, 1. Auflage, Simmerath 1999

Zitiert: Chauvistré

Chemnitr, Jürgen/ Johnigk, Franz

Rechtsberatungsgesetz: Kommentar, 11. Auflage, Münster 2003 
Coeppicus, Rolf

Freiheit zur Krankheit?, BtPrax 1999, 130 ff.

Coeppicus, Rolf

Sachfragen des Betreuungs- und Unterbringungsrechts, Stuttgart 2000

Zitiert: Coeppicus, Sachfragen

Coeppicus, Rolf

5460 DM jährlich für Betreuungsfahrten zu den Eltern, BtPrax 2003, 164 ff.

Coeppicus, Rolf

Sterbehilfe, Patientenverfügung und Vorsorgevollmacht: Verbindlichkeit,

Muster, Umsetzung. Ein Ratgeber für Rechtssicherheit am Lebensende,

1. Auflage, Essen 2006

Zitiert: Coeppicus, Sterbehilfe

Cypionka, Bertram

Die Auswirkungen des Betreuungsgesetzes auf die Praxis des Notars, DNotZ 1991, $571 \mathrm{ff}$.

Cypionka, Bertram

Fortfall der Entmündigung Volljähriger - Auswirkungen auf den Rechtsverkehr, NJW 1992, 207 ff.

Damrau, Jürgen/ Zimmermann, Walter (Hrsg.)

Betreuungsrecht: Kommentar zum materiellen und formellen Recht, 3. Auflage, Stuttgart 2001

Deinert, Horst

Betreuungszahlen 2005, BtPrax 2007, 3 ff.

Deinert, Horst/ Lütgens, Kay

Die Vergütung des Betreuers. Handbuch der Vergütungs- und Aufwendungsregelungen, 4. Auflage Köln 2005

Deutsch, Erwin

Verfassungszivilrecht bei der Sterbehilfe, NJW 2003, 1567 ff.

Deutsche Hospizstiftung

Wie denken die Deutschen über Patientenverfügungen?, November 2005, online veröffentlicht unter

http://www.hospize.de/ftp/tns_studie_05.pdf (Stand: Dezember 2009)

Diekmann, Albrecht

Empfiehlt es sich, das Entmündigungsrecht, das Recht der Vormundschaft und der Pflegschaft über Erwachsene sowie das Unterbringungsrecht neu zu ordnen?, JZ 1988, 789 ff.

Diekmann, Andrea

Neue Verfahrensvorschriften in Betreuungssachen nach dem FamFG ein Überblick, BtPrax 2009, 149 ff.

Dodegge, Georg/ Roth, Andreas

Systematischer Praxiskommentar Betreuungsrecht, 2. Auflage, Köln 2005 
Dodegge, Georg

Die Entwicklung des Betreuungsrechts bis Anfang Juni 2006, NJW 2006, 2670 ff.

Dolzer, Rodolf (Hrsg.)

Bonner Kommentar zum Grundgesetz: gegründet 1950, Heidelberg 1991, Loseblattsammlung Stand: Februar 2009

Zitiert: BK/ Bearbeiter

Dölle, Hans

Familienrechtliche Darstellung des deutschen Familienrechts mit rechtsvergleichenden Hinweisen Band II, Karlsruhe 1965

Dreier, Horst (Hrsg.)

Grundgesetz Kommentar, Band 1, 2. Auflage, Tübingen 2008

Eisenbart, Bettina

Patienten-Testament und Stellvertretung in Gesundheitsangelegenheiten.

Alternativen zur Verwirklichung der Selbstbestimmung im Vorfeld des Todes, 2. Auflage, Baden-Baden 2000

Epping, Volker/ Hillgruber, Christian (Hrsg.)

Beck'scher OnlineKommentar zum Grundgesetz, Stand: 01.10.2008, Edition 2

Epple, Dieter

Die Betreuungsverfügung, BWNotZ 1992, 27 ff.

Epple, Dieter

Einfluß der Betreuungsverfügung auf das Verfahren, die Führung und Erman

Überwachung der Betreuung, BtPrax 1993, 156 ff.

Handkommentar zum Bürgerlichen Gesetzbuch, Westermann, Harm Peter (Hrgs.)

11. Auflage, Köln 2004, zitiert: Ermann/ Holz̧hauer

12. Auflage, Köln 2008, zitiert: Erman/Roth

Feil, Erich/ Marent, Karl-Heinz (Hrgs.)

Familienrechtskommentar zum ABGB, Wien 2007

Flume, Werner

Allgemeiner Teil des Bürgerlichen Rechts. Zweiter Band: Das Rechtsgeschäft, 4. Auflage, Berlin, Heidelberg, New York 1992

Friedrichs, Hans-Joachim

Das neue Betreuungsgesetz, MDR 1992, $5 \mathrm{ff}$.

Fröschle, Tobias (Hrsg.)

Praxiskommentar Betreuungs- und Unterbringungsverfahren:

Frost, Andreas

FGG, KostO, RpflG, BtBG, Köln 2007

Arztrechtliche Probleme des neuen Betreuungsrechtes. Eine Betrachtung der \S 1901, 1904 und 1905 BGB unter besonderer Berücksichtigung der Einwilligung in ärztliche Maßnahmen, Heidelberg 1994 
Ganner, Michael

Selbstbestimmung im Alter. Privatautonomie für alte und pflegebedürftige

Menschen in Österreich und Deutschland, Wien 2005

Ganner, Michael

Das österreichische Sachwalterrecht (Teil 1), BtPrax 2007, 238 ff.

Das österreichische Sachwalterrecht - eine Erfolgsgeschichte? (Teil 2), BtPrax 2008, 3 ff.

Geckle, Gerhard

Patientenverfügung und Testament, 2. Auflage, Freiburg 2008

Gernhuber, Joachim/ Coester-Waltjen, Dagmar

Familienrecht, 5. völlig neu bearbeitete Auflage, München 2006

Grell, Thomas

Qualifikation des Verfahrenspflegers, Rpfleger 1993, $321 \mathrm{ff.}$

Grimotz, Herbert

Der ,betreute' Stifter - Zur Zulässigkeit von Stiftungen entsprechend dem

Willen des Betreuten, ZEV 2005, 338 ff.

Harm, Uwe

Die ,Angelegenheiten' einer volljährigen Person im Sinne des Betreuungsgesetzes, Rpfleger 1998, 89 ff.

Harm, Uwe

Die ,Wohnungsauflösung'. Gerichtliche Aufsicht und Genehmigungsver-

fahren, Rpfleger 2002, 59 ff.

Hartmann, Tanja

Patientenverfügung und Psychiatrische Verfügung - Verbindlichkeit für den Arzt?, NStZ 2000, 113 ff.

Heinken, Karl

Die Ordnung des gesamten Rechtsberatungswesens in Deutschland. Eine

Gesamtdarstellung der Gesetze, Verordnungen und Verbandsabkommen über die Rechtslage, Heide in Holstein 1939

Holghauer, Heinz

Der Umfang gerichtlicher Kontrolle privatrechtlicher Unterbringung nach

\1906 BGB i.d.F. des Betreuungsgesetzes, FuR 1992, 249 ff.

Holzhauer, Heinz

Schenkungen aus dem Vermögen Betreuter, FamRZ 2000, 1063 ff.

Holzhauer, Heinz/ Reinicke, Michael

Betreuungsrecht: Eine Kommentierung der bürgerlich-rechtlichen und der verfahrensrechtlichen Vorschriften des neuen Betreuungsrechts, Münster 1993

Ill-Groß, Manuela/ Sträßner, Heinz R.

Patiententestament, Betreuungsverfügung und Vorsorgevollmacht in der Praxis, PflR 1999, 126 ff. 
Ingelfinger, Ralph

Patientenautonomie und Strafrecht bei der Sterbebegleitung, JZ 2006, $821 \mathrm{ff}$.

Jansen, Paul (Begr.)/ Schuckmann von, Hans-Joachim/ Sonnenfeld, Susanne (Hrsg.)

Gesetz über die Angelegenheiten der freiwilligen Gerichtsbarkeit Großkommentar, 2. Band $\int \$ 35$ - 70n FGG, 3. Auflage, Berlin 2005

Zitiert: Jansen/ Bearbeiter

Jülicher, Hans-Oskar/ Klinger, Bernhard F.

Patientenverfügung und Vorsorgevollmacht, 2. Auflage Köln 2005

Jurgeleit, Andreas (Hrsg.)

Betreuungsrecht, Handkommentar, Baden-Baden 2006

Jürgens, Andreas (Hrsg.)

Betreuungsrecht. Kommentar zum materiellen Betreuungsrecht, zum

Verfahrensrecht und zum Vormünder- und Betreuervergütungsgesetz,

3. Auflage, München 2005

Jürgens, Andreas/ Kröger, Detlef/ Marscbner, Rolf/Winterstein, Peter

Betreuungsrecht kompakt. Systematische Darstellung des gesamten

Betreuungsrechts, 6. Auflage, München 2007

Justiżministerium Freistaat Thüringen

Wie kann ich vorsorgen?, Erfurt 2008

Justizministerium Mecklenburg Vorpommern

Vorsorgevollmacht und Betreuungsrecht, Schwerin 2003

Justizministerium Niedersachsen

Das Betreuungsrecht, Hannover 2005

Keidel, Theodor (Begr.)/ Kuntze, Joachim/ Winkler, Karl

Freiwillige Gerichtsbarkeit. Kommentar zum Gesetz über die Angelegen-

heiten der freiwilligen Gerichtsbarkeit, 15. Auflage, München 2003

Keidel, Theodor (Begr.)/ Engelhardt, Helmut/ Sternal, Werner

FamFG. Kommentar zum Gesetz über das Verfahren in Familiensachen und die Angelegenheiten der freiwilligen Gerichtsbarkeit, 16. Auflage, München 2009

Keilbach, Heinz

Vorsorgevollmacht zur Wahrung der Selbstbestimmung bei Krankheit, im

Alter und am Lebensende, FamRZ 2003, 969 ff.

Keim, Benno

Das notarielle Beurkundungsverfahren, München 1990

Kemper, Rainer

Das Betreuungsrecht in der gerichtlichen Praxis. Übersicht über die von

Juni 1992 bis Juni 1994 veröffentlichte Rechtsprechung, FuR 1994, 677 ff.

Kirsch, Matthias

Die Vergütung des Verfahrenspflegers, Rpfleger 1992, 379 ff. 
Klinger, Bernhard F. (Hrsg.)

Patientenverfügung und Vorsorgevollmacht. Was Ärzte und Bevollmächtigte für Sie in einem Notfall tun sollen, Wien 2005

Knieper, Judith

Neue Vergütungsregelungen für Verfahrenspfleger nach dem BtÄndG, JurBüro 1998, 289 ff.

Knittel, Bernhard

Betreuungsgesetz (BtG). Gesetz zur Reform des Rechts der Vormundschaft und Pflegschaft für Volljährige. Kommentar, Stand: 01. Oktober 2008, Köln

Köller, Regine/ Sellin, Christine/ Engels, Dietrich

Evaluation des 2. Betreuungsrechtsänderungsgesetzes (2. BtÄndG) - im Auftrag des Bundesministeriums der Justiz - Zwischenbericht 2007, Institut für Sozialforschung und Gesellschaftspolitik, Köln 2007

Kollmer, Norbert

Selbstbestimmung im Betreuungsrecht, München 1992

Korte, Tamayo

Altern - ein Risiko für Autonomie?, FPR 2004, 643 ff.

Koziol, Helmut/ Bydlinski, Peter/ Bollenberger, Raimund (Hrsg.)

Krauß, Dieter

ABGB Kommentar, 2. Auflage, Wien, New York 2007

Die Änderungen des Betreuungsrechts durch das 2. Gesetz zur Änderung

des Betreuungsrechts, BWNotZ 2006, 35 ff.

Kremzow, Friedrich Wilhelm

Österreichisches Sachwalterrecht. Eine kommentierte Darstellung des

Bundesgesetzes vom 02.02.1983 über die Sachwalterschaft für behinderte

Personen, Eisenstadt 1984

Lakotta, Beate

Das Leiden der Anderen, S. 164 ff. Der Spiegel Nr. 48/ 24.11.2008

Langenfeld, Andrea

Vorsorgevollmacht, Betreuungsverfügung und Patiententestament nach dem neuen Betreuungsrecht, Konstanz 1994

Larenz, Karl/ Wolf, Manfred

Allgemeiner Teil des Bürgerlichen Rechts, 9. Auflage, München 2004

Laufs, Adolf / Katzenmeier, Christian/ Lipp, Volker (Hrsg.)

Arztrecht 6. Auflage, München 2009

Lipp, Volker

Freiheit und Fürsorge: Der Mensch als Rechtsperson. Zu Funktion und

Stellung der rechtlichen Betreuung im Privatrecht. Tübingen 2000

Zitiert: Lipp 
Lipp, Volker

Rechtliche Aspekte stellvertretender Entscheidungen bei „passiver Sterbehilfe", in: May, Arnd T./ Geißendörfer, Sylke E./ Simon, Alfred/ Strätling, Meinolfus (Hrsg.), Passive Sterbehilfe: besteht gesetzlicher Regelungsbedarf? Impulse aus einem Expertengespräch der Akademie für Ethik in der Medizin e.V., Münster, Hamburg, London 2002, S. 37 ff.

Zitiert: May/ Geißendörfer/ Simon/ Strätling/ Lipp

Lipp, Volker

Die Entscheidung des BGH zur Sterbehilfe, BtPrax 2004, 18 ff.

Lipp, Volker

Sterbehilfe und Patientenverfügung, FamRZ 2004, 317 ff.

Lipp, Volker

Patientenautonomie und Lebensschutz. Zur Diskussion um eine gesetzliche Regelung der „Sterbehilfe“, Göttingen 2005

Zitiert: Lipp, Patientenautonomie

Lipp, Volker

Die Betreuungsverfügung als Instrument privater Vorsorge, in: Sonnenfeld, Susanne (Hrsg.), Nichtalltägliche Fragen aus dem Alltag des Betreuungsrechts: Festschrift für Werner Bienwald zum 70. Geburtstag am 6. Juli 2006, Bielefeld 2006, S. 177 ff.

Lipp, Volker

Betreuung und Zwangsbehandlung, JZ 2006, 661 ff.

Lipp, Volker

Rechtliche Betreuung und das Recht auf Freiheit, BtPrax 2008, 51 ff.

Lipp, Volker (Hrsg.)

Handbuch der Vorsorgeverfügungen. Vorsorgevollmacht - Patientenverfügung - Betreuungsverfügung. München 2009

Zitiert: Lipp, Vorsorgeverfügungen

Lipp, Volker/ Klein, Frederike C.A.

Patientenautonomie und „Sterbehilfe“ - Stand der aktuellen Debatte, FPR 2007, 56 ff.

Loozv., Carola

Die Lebensdecke ist nicht kochfest! - Plädoyer für eine Betreuungsverfügung, BtPrax 2002, $179 \mathrm{ff}$.

Martin, Anja M.

Die Betreuung mit dem Aufgabenkreis Gesundheitssorge,

Frankfurt a.M. 2002

Zitiert: Martin

Maunz, Theodor von/ Dürig, Günther/ Herzog, Roman (Hrsg.)

Kommentar zum Grundgesetz für die Bundesrepublik Deutschland vom 23. Mai 1949, Loseblattsammlung, München, Stand: Oktober 2008

Zitiert: Maunz/ Dürig/ Bearbeiter 
Maurer, Ewald

Das österreichische Sachwalterrecht in der Praxis. Sachwaltergesetz in der Fassung des Sachwalterrechtsänderungsgesetzes 2006, 3. Auflage, Wien 2007

Mayer, Karl-Georg

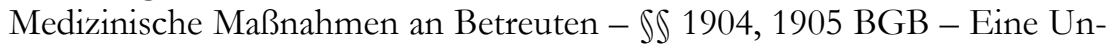
tersuchung aus zivilrechtlicher Sicht. Würzburg 1995

Zitiert: Mayer

Meier, Sybille M.

Zu den Aufgaben der Betreuungsbehörde, BtPrax 2005, 82 ff. Milzer, Lutz

Die adressengerechte Vorsorgevollmacht, NJW 2003, 1836 ff.

Motive zu dem Entwurfe eines Bürgerlichen Gesetzbuches für das Deutsche Reich, Berlin und Leipzig 1888.

Müller, Gabriele

Betreuung und Geschäftsfähigkeit, Bielefeld 1998

Müller, Gabriele/ Renner, Thomas

Betreuungsrecht und Vorsorgeverfügungen in der Praxis,

1. Auflage, Münster 2005/ 2. Auflage, Münster 2008

Müller-Freienfels, Wolfram

Die Altersvorsorge-Vollmacht. Studie zur Vollmachterteilung über Minderungen der Geschäftsfähigkeit hinaus, in: Horn, Norbert (Hrsg.), Europäisches Rechtsdenken in Geschichte und Gegenwart. Festschrift für Helmut Coing zum 70. Geburtstag, Band 2, München 1982, S. 395 ff.

Münchener Kommentar zum Bürgerlichen Gesetzbuch

Rebmann, Kurt/ Säcker, Franz Jürgen/ Rixecker, Roland (Hrsg.)

Band 1 Allgemeiner Teil \$S 1 - 240, ProstG, 5. Auflage, München 2006

Band 8 Familienrecht II SS 1589 - 1921, SGB VIII, 5. Auflage, München 2008

Zitiert: MünchKommBGB/ Bearbeiter

Münchener Kommentar zur Zivilprozessordnung

Rauscher, Thomas / Wax, Peter/ Wenzel, Joachim (Hrsg.)

Band 4 Gesetz über das Verfahren in Familiensachen und in den Angelegenheiten der freiwilligen Gerichtsbarkeit (FamFG), München 2010

Zitiert: MünchKommZPO/ Bearbeiter

Neubausen, Matthias

Rechtsgeschäfte mit Betreuten, RNotZ 2003, 157 ff. 
Palandt, Otto

Bürgerliches Gesetzbuch, Kurzkommentar, bearbeitet von Bassenge, Peter/ Brudermüller, Gerd/ Diederichsen, Uwe/ Edenhofer, Wolfgang/ Grünberg, Christian/ Heldrich, Andreas/ Heinrichs, Helmut/ Sprau, Hartwig/ Putzo, Hans / Weidekaff, Walter, 69. Auflage, München 2009

Zitiert: Palandt/ Bearbeiter

63. Auflage, München 2004, zitiert: Palandt/ Bearbeiter, 63. Auflage

55. Auflage, München 1996, zitiert: Palandt/ Bearbeiter, 55. Auflage

Pankoke-Schenk, Monika

Vormundschaftsrecht und Pflegschaft über Volljährige - die Reformbedürftigkeit des geltenden Rechts und die geplanten Neuregelungen aus der Sicht freigemeinnütziger Vormundschafts-/ Pflegschaftsarbeit, NDV 1989, $49 \mathrm{ff}$.

Pardey, Karl-Dieter

Betreuung Volljähriger: Hilfe oder Eingriff - Anspruch und Leistungsfähigkeit des Betreuungsrechts im Lichte des Grundgesetzes Sarstedt 1989

Pardey, Karl-Dieter

Zur Zulässigkeit drittschützender freiheitsentziehender Maßnahmen nach \1906 BGB, FamRZ 1995, 713 ff.

Pawlowski, Hans-Martin

Patientenverfügung, Vorsorgevollmacht und Betreuungsverfügung. Zur rechtlichen Vorsorge für das Alter, in: Sonnenfeld, Susanne (Hrsg.) Nichtalltägliche Fragen aus dem Alltag des Betreuungsrechts: Festschrift für Werner Bienwald zum 70. Geburtstag am 06. Juli 2006, Bielefeld 2006, S. $215 \mathrm{ff}$.

Pawlowski, Hans-Martin/ Smid, Stefan

Freiwillige Gerichtsbarkeit. Ein Lehrbuch. Köln, Berlin, Bonn, München 1993

Perau, Guido

Betreuungsverfügung und Vorsorgevollmacht, MittRhNotK 1996, 285 ff. Peters, Bernd

Die Betreuung Volljähriger. Die Reform des zivilrechtlichen Fürsorgerechts für Volljährige aus der Sicht des Betreuers, Bayreuth 1992

Pieroth, Bodo

Die Verfassungsmäßigkeit der Sterilisation Einwilligungsunfähiger gemäß dem Entwurf für ein Betreuungsgesetz, FamRZ 1990, 117 ff.

Pobl, Kay-Thomas

Verfahrenspflegschaft, BtPrax 1992, 19 ff.

Prinz von Sachsen Gessaphe, Karl August

Der Betreuer als gesetzlicher Vertreter für eingeschränkt Selbstbestimmungsfähige, Tübingen 1999 
Rauscher, Thomas

Familienrecht, 2. Auflage, Heidelberg 2008

Rennen, Günter/ Caliebe, Gabriele

Rechtsberatungsgesetz mit Ausführungsverordnungen, 2. Auflage, München 1992

Rieger, Gregor

Machtlos trotz Vollmacht? Materiell- und verfahrensrechtlicher Schutz von Vorsorgevollmacht und Betreuungsverfügung, in: Hofer, Sibylle/ Klippel, Diethelm/ Walter, Ute (Hrsg.), Perspektiven des Familienrechts: Festschrift Dieter Schwab zum 70. Geburtstag am 15. August 2005, Bielefeld 2005, S. 1043 ff.

Rink, Jürgen

Anforderungen an das Vormundschaftsgericht und die Vormundschaftsrichter aufgrund des Betreuungsgesetzes, FuR 1990, 253 ff.

Rogalla, Catharina

Mehr Rechtsschutz durch den Verfahrenspfleger, BtPrax 1993, 146 ff.

Roth, Andreas

Die Verbindlichkeit der Patientenverfügung und der Schutz des Selbstbestimmungsrechts, JZ 2004, $494 \mathrm{ff}$.

Röthel, Anne

Altersvorsorgevollmacht und Betreuungsverfügung, in: Hausmann, Rainer/ Hobloch, Gerhard (Hrsg.), Handbuch des Erbrechts, Berlin 2008, S. $1134 \mathrm{ff}$.

Zitiert: Hausmann/ Hohloch/ Röthel

Rudolf, Michael/ Bittler, Jan

Vorsorgevollmacht, Betreuungsverfügung, Patientenverfügung, Bonn 2000

Schauer, Martin

Schwerpunkte des Sachwalterrechts-Änderungsgesetzes (SWRÄG 2006) Teil 1, ÖJZ 2007, 173 ff.

Schlöpke, Stephan

Vergütung von Verfahrenspflegern in Unterbringungssachen, Rpfleger 1993, 435 ff.

Schmidt, Gerd/ Böcker, Felix (Hrsg.)

Betreuungsrecht. Eine systematische Einführung aus juristischer und psychiatrischer Sicht, 2. Auflage, München 1993

Schrader, Peter

„Selbstbewusst die Zukunft gestalten, solange ich gesund bin“ Die Vorsorgevollmacht - die Betreuungsverfügung - die Patientinnen-/ Patientenverfügung, Die Schiedsamtszeitung (SchAZ) 2002, 49 ff.

Schumacher, Ulrich

Hypertrophie der Verfahrensgarantien im Betreuungsgesetz-Entwurf?, ZRP 1989, $7 \mathrm{ff}$. 
Schumacher, Ulrich

Wohnraummiete und Betreuung, WuM 2003, 190 ff.

Schwab, Dieter

Empfiehlt es sich, das Entmündigungsrecht, das Recht der Vormundschaft und der Pflegschaft über Erwachsene sowie das Unterbringungsrecht neu zu ordnen?, Referat auf dem 57. Deutschen Juristentag Band II, in: Verhandlungen des 57. Deutschen Juristentags in Mainz 1988, München 1988, S. K 8 ff.

Schwab, Dieter

Strukturfragen des geplanten Betreuungsrechts, in: Schwab, Dieter (Hrsg.), Staat, Kirche, Wissenschaft in einer pluralistischen Gesellschaft, Festschrift zum 65. Geburtstag von Paul Mikat, Berlin 1989, S. 881 ff.

Schwab, Dieter

Das neue Betreuungsrecht. Bericht über die verabschiedete Fassung des Betreuungsgesetzes (BtG), FamRZ 1990, 681 ff.

Schwab, Dieter

Probleme des materiellen Betreuungsrechts, FamRZ 1992, 493 ff.

Schwab, Dieter

Probleme der Vorsorgevollmacht, in: Bauer, Hans-Joachim (Hrsg.), Versorgung und Vorsorge. Familienrecht in Bewegung, Berlin 2004, S. 27 ff.

Schwimann, Michael (Hrgs.)

ABGB Praxiskommentar. Ergänzungsband und Gesamtwortverzeichnis für alle 7 Bände, 3. Auflage, Wien 2007

Sellin, Christine/ Engels, Dietrich

Qualität, Aufgabenverteilung und Verfahrensaufwand bei rechtlicher Betreuung - mit einem Rechtsgutachten von Prof. Dr. Heinz Holzhauer

Untersuchung im Auftrag des Bundesministeriums der Justiz (Hrgs.) Köln 2003

Simon, Alfred/ Meran, Johannes G./ Fangerau, Heiner

Patientenverfügungen als Instrument der Patientenselbstbestimmung,

Der Hautarzt 2004, $721 \mathrm{ff}$.

Soergel, Hs. Theodor (Begr.)/ Siebert, Wolfgang (Hrsg.)

Bürgerliches Gesetzbuch mit Einführungsgesetz und Nebengesetzen.

Band 20 Familienrecht 4 SS 1741 - 1921, 13. Auflage, Stuttgart 2000

Sonnenfeld, Susanne

Das 2. Bt ̈̈ndG, FamRZ 2005, 941 ff.

Spickhoff, Andreas

Die Patientenautonomie am Lebensende: Ende der Patientenautonomie?

- Zur Feststellbarkeit und Durchsetzbarkeit des realen oder hypotheti-

schen Willens des Patienten, NJW 2000, 2297 ff.

Spickhoff, Andreas

Autonomie und Heteronomie im Alter, in: AcP Bd. 208 (2008), S. 345 ff. 
Spanl, Reinhold

Ergänzungsbetreuung und Gegenbetreuung - Neue Begriffe des Betreuungsrechts, Rpfleger 1992, 142 ff.

Ständeke-Otto, Edeltraud

Rechtsanwälte als Verfahrenspfleger, BtPrax 1993, 16 ff.

Statistisches Bundesamt (Hrsg.)

Bevölkerung Deutschland bis 2050, 11. koordinierte

Bevölkerungsvorausberechnung. Wiesbaden 2006

Staudinger, J. von

Kommentar zum Bürgerlichen Gesetzbuch mit Einführungsgesetz und

Nebengesetzen

Buch 1 Allgemeiner Teil SS 164 - 240 Allgemeiner Teil 5, 13. Auflage, Berlin 2004

Buch 4 Familienrecht \$S 1773 - 1895 Anhang zu $\int S 1773$ - 1895 (KJHG)

Vormundschaftsrecht, Neubearb., Berlin 2004

Buch 4 Familienrecht \S 1896 - 1921 Rechtliche Betreuung und Pflegschaft, Neubearb., Berlin 2006

Taupitz, Jochen

Von der entrechteten Bevormundung zur helfenden Betreuung: Das neue Betreuungsgesetz, JuS 1992, 9 ff.

Taupitz, Jochen

„Empfehlen sich zivilrechtliche Regelungen zur Absicherung der Patientenautonomie am Ende des Lebens?" Gutachten A für den 63. Deutschen Juristentag,

in: Verhandlungen des 63. Deutschen Juristentages Band I, München 2000, S. A 5 ff.

Thomas, Heinz/ Putzo, Hans/ Reichold, Klaus/ Hüßtege, Rainer

Zivilprozessordnung: mit Gerichtsverfassungsgesetz, den Einführungsgesetzen und europarechtlichen Vorschriften; Kommentar, 29. Auflage, München 2008

Ublenbruck, Wilhelm

Selbstbestimmtes Sterben durch Patienten-Testament, Vorsorgevollmacht, Betreuungsverfügung, Berlin 1997

Zitiert: Uhlenbruck, Selbstbestimmtes Sterben

Ublenbruck, Wilhelm

Patiententestament, Betreuungsverfügung und Vorsorgevollmacht: zur

Selbstbestimmung im Vorfeld des Todes, 3. Auflage, Dortmund 1998

Zitiert: Uhlenbruck, Patiententestament

Ublenbruck, Wilhelm

Bedenkliche Aushöhlung der Patientenrechte durch die Gerichte, NJW 2003, 1710 ff. 
Verell, Thorsten

„Patientenautonomie und Strafrecht bei der Sterbebegleitung“ Gutachten C zum 66. Deutschen Juristentag

in: Verhandlungen des 66. Deutschen Juristentages Band I, München 2006 S. C. $5 \mathrm{ff}$.

van Oorschot, B.

Patientenverfügung aus Patientensicht. Ergebnisse einer Befragung von palliativ behandelten Tumorpatienten, Ethik in der Medizin 2004, 112 ff.

Vennemann, Ulrich

Der Betreuungsrichter an der (zu) kurzen Leine - zur Verhältnismäßigkeit im (Betreuungs-)verfahrensrecht, BtPrax 1994, 93 f.

Verbraucherzentrale $N R W$ e.V.

Patientenverfügung, Vorsorgevollmacht und Betreuungsverfügung, 10. Auflage, Düsseldorf 2008

Viefhues, Wolfram (Hrsg.)

Juris Praxiskommentar BGB, Buch 4 - Familienrecht, 4. Auflage, Saarbrücken 2008

Zitiert: juris PK-BGB/ Bearbeiter

Wagenitr, Thomas/Schwab, Dieter

Familienrechtsreformkommentar Bielefeld 1998

Zitiert: FamRefK/ Bearbeiter

Walter, Ute

Die Vorsorgevollmacht, Bielfeld 1997

Wesche, Otto

Die Geldverwaltung durch Betreuer, BtPrax 2003, 56 ff.

Wigge, Peter

Arztrechtliche Fragen des Unterbringungsrechts, MedR 1996, 291 ff.

Winkler, Matthias

Vorsorgeverfügungen (Patientenverfügung Vorsorgevollmacht, Betreuungs- und Organverfügung), 3. Auflage, München 2007

Zen₹, Giesela/ von Eicken, Barbara/ Ernst, Ellen/ Hofmann, Cornelia

Vormundschaft und Pflegschaft für Volljährige. Eine Untersuchung zur

Praxis und Kritik des geltenden Rechts, Bundesministerium der Justiz (Hrsg.), Köln 1987

Zitiert: Zenz/ Eicken/ Ernst/ Hofmann

Zierl, Hans Peter

Sachwalterrecht Kurzkommentar mit Musterteil und Paragraphenübersicht, Sachwalterrechts-Änderungsgesetz 2006, PatientenverfügungsGesetz, Heimaufenthaltsgesetz/Heimvertretungsgesetz, Außerstreitgesetz, Wien 2007

Zimmermann, Walter

Das neue Verfahren in Unterbringungssachen, FamRZ 1990, 1308 ff. 
Zimmermann, Walter

Das neue Verfahren in Betreuungssachen, FamRZ 1991, 270 ff.

Zimmermann, Walter

Probleme des neuen Betreuervergütungsrechts, FamRZ 1999, 630 ff.

Zimmermann, Walter

Die Rechtsprechung zur Betreuungsvergütung, FamRZ 2002, 1373 ff.

Zimmermann, Walter

Vorsorgevollmacht, Betreuungsverfügung, Patientenverfügung für die

Praxisberatung, Berlin 2007

Zitiert: Zimmermann

Zimmermann, Walter

Das neue FamFG Verfahrensrecht, Rechtsmittel, Familiensachen,

Betreuung, Unterbringungssachen, Nachlasssachen und Kosten, München 2009

Zitiert: Zimmermann FamFG

Zöller, Richard

Zivilprozessordnung mit FamFG (\$S 1 - 185, 200 - 270, 433 - 48) und Gerichtverfassungsgesetz, den Einführungsgesetzen, mit Internationalem Zivilprozessrecht, EG-Verordnungen, Kostenanmerkungen Kommentar, 28. Auflage, Köln 2010 



\section{Anhang: Auszug aus dem ABGB}

Auszug aus dem Fünften Hauptstück des Österreichischen Allgemeinen Bürgerlichen Gesetzbuchs in der Fassung vom 01.12.2009:

Von der Sachwalterschaft, der sonstigen gesetzlichen Vertretung und der Vorsorgevollmacht $\$ \mathbb{S} 268 \mathrm{ff}$. ABGB

\section{Besondere Vorschriften für die Sachwalterschaft}

\section{a) Auswahl des Sachwalters} $\S 279$ ABGB

(1) Bei der Auswahl des Sachwalters ist besonders auf die Bedürfnisse der behinderten Person und darauf Bedacht zu nehmen, dass der Sachwalter nicht in einem Abhängigkeitsverhältnis oder in einer anderen engen Beziehung zu einer Krankenanstalt, einem Heim oder einer sonstigen Einrichtung steht, in der sich die behinderte Person aufhält oder von der sie betreut wird. Wünsche der behinderten Person, insbesondere solche, die sie vor Verlust der Geschäftsfähigkeit und Einsichts- und Urteilsfähigkeit geäußert hat (Sachwalterverfügung), und Anregungen nahe stehender Personen sind zu berücksichtigen, sofern sie dem Wohl der behinderten Person entsprechen. 
(2) Einer behinderten Person ist eine geeignete, ihr nahe stehende Person zum Sachwalter zu bestellen. Wird eine behinderte Person volljährig, so ist ein bisher mit der Obsorge betrauter Elternteil zum Sachwalter zu bestellen, sofern dies dem Wohl der behinderten Person nicht widerspricht.

(3) Ist eine geeignete, nahe stehende Person nicht verfügbar, so ist ein geeigneter Verein mit dessen Zustimmung zum Sachwalter zu bestellen. Kommt auch ein Verein nicht in Betracht, so ist nach Maßgabe des \274 Abs. 2 ein Rechtsanwalt (Rechtsanwaltsanwärter) oder Notar (Notariatskandidat) oder eine andere geeignete Person mit deren Zustimmung zu bestellen.

(4) Ein Rechtsanwalt (Rechtsanwaltsanwärter) oder Notar (Notariatskandidat) ist vor allem dann zum Sachwalter zu bestellen, wenn die Besorgung der Angelegenheiten vorwiegend Rechtskenntnisse erfordert, ein geeigneter Verein vor allem dann, wenn sonst besondere Anforderungen mit der Sachwalterschaft verbunden sind.

(5) Eine Person darf nur so viele Sachwalterschaften übernehmen, wie sie unter Bedachtnahme auf die Pflichten eines Sachwalters, insbesondere jene zur persönlichen Kontaktnahme, ordnungsgemäß besorgen kann. Es wird vermutet, dass eine Person - ausgenommen ein geeigneter Verein - insgesamt nicht mehr als fünf, ein Rechtsanwalt oder Notar nicht mehr als 25 Sachwalterschaften übernehmen kann; Sachwalterschaften zur Besorgung einzelner Angelegenheiten bleiben dabei außer Betracht.

\section{b) Geschäftsfähigkeit der behinderten Person $\int 280$ ABGB}

(1) Die behinderte Person kann innerhalb des Wirkungskreises des Sachwalters ohne dessen ausdrückliche oder stillschweigende Einwilligung rechtsgeschäftlich weder verfügen noch sich verpflichten.

(2) Schließt die behinderte Person im Rahmen des Wirkungskreises des Sachwalters ein Rechtsgeschäft, das eine geringfügige Angelegenheit des täglichen Lebens betrifft, so wird dieses Rechtsgeschäft mit der Erfüllung der die behinderte Person treffenden Pflichten rückwirkend rechtswirksam.

\section{c) Berücksichtigung des Willens und der Bedürfnisse der behinderten Person \281 ABGB}

(1) Der Sachwalter hat danach zu trachten, dass die behinderte Person im Rahmen ihrer Fähigkeiten und Möglichkeiten ihre Lebensverhältnisse nach ihren Wünschen und Vorstellungen gestalten kann.

(2) Die behinderte Person hat das Recht, von beabsichtigten, ihre Person oder ihr Vermögen betreffenden wichtigen Maßnahmen vom Sachwalter rechtzeitig verständigt zu werden und sich hiezu, wie auch zu anderen Maßnahmen, in angemes- 
sener Frist zu äußern; diese Äußerung ist zu berücksichtigen, wenn der darin ausgedrückte Wunsch dem Wohl der behinderten Person nicht weniger entspricht.

(3) Ist der Sachwalter mit der Verwaltung des Vermögens oder des Einkommens der behinderten Person betraut, so hat er diese vorrangig zur Deckung der den persönlichen Lebensverhältnissen entsprechenden Bedürfnisse der behinderten Person zu verwenden.

(4) Ist das Wohl der behinderten Person gefährdet, so hat das Gericht jederzeit, von wem immer es angerufen wird, die zur Sicherung ihres Wohles nötigen Verfügungen zu treffen. 



\section{Lebenslauf}

Als Tochter der Erzieherin Inge Klein, geb. Wagner und des Bauzeichners Robert Klein wurde ich am 25.04.1981 in Kassel geboren.

Nach dem Besuch der Grundschule Warmetal Obermeiser und der GustavHeinemann Gesamtschule Hofgeismar besuchte ich von 1997 bis 2000 das Oberstufengymnasium Albert-Schweitzer in Hofgeismar. Am 06.06.2000 erwarb ich dort die Allgemeine Hochschulreife.

Ab dem Wintersemester 2000/2001 studierte ich Rechtswissenschaft an der Georg-August-Universität Göttingen. Das Studium setze ich im Wintersemester 2002/2003 für sechs Monate an der Universitá degli Studi di Perugia in Italien fort. Am 12.12.2005 schloss ich in Göttingen mein Studium der Rechtswissenschaft mit dem ersten juristischem Staatsexamen und dem Diplom ab.

Vom 01.04.2006 bis zum 31.03.2009 war ich als wissenschaftliche Assistentin von Prof. Dr. Volker Lipp am Lehrstuhl für Bürgerliches Recht, Rechtsvergleichung und Zivilprozessrecht an der Georg-August-Universität Göttingen tätig. Mein dortiger Arbeitsschwerpunkt lag im Familienrecht, insbesondere dem Betreuungsrecht. In dieser Zeit entstand die vorliegende Dissertation. 
Seit dem 01.04.2009 bin ich Rechtsreferendarin am Landgericht Lübeck in Schleswig-Holstein.

Im Sommer 2010 habe ich meinen jetzigen Ehemann Dr. Florian Faupel geheiratet.

Lübeck, im Oktober 2010

Frederike C.A. Faupel 
Mit steigendem Alter nehmen degenerative Erkrankungen zu, damit können Beschränkungen der geistigen und körperlichen Fähigkeiten einhergehen. Vermindert sich die mentale Leistung, reduziert sich häufig auch die Fähigkeit, eigene Entscheidungen zu treffen bzw. getroffene Entscheidungen umzusetzen. Zunehmend stellt sich in diesen Fällen die Frage, wer anstelle des Betroffenen die notwendigen rechtlichen Angelegenheiten übernimmt und nach welchen Kriterien die erforderliche Hilfe erfolgt. Das Gesetz regelt dies gemäß $\S \S 1896$ ff. BGB mit der Betreuung.

Gemäß den §§ 1901, 1897, 1901c BGB kann jeder Einzelne im Rahmen einer Betreungsverfügung im Vorfeld das $\mathrm{Ob}$ und Wie seiner eigenen möglichen Betreuung festlegen. Er kann unter anderem gemäß § 1897 Abs. 4 BGB die Person seines Betreuers bestimmen und diesem gemäß § 1901 Abs. 1 und 2 BGB Vorgaben zur Führung der Betreuung geben. Die rechtliche Grundlage dafür ist das Selbstbestimmungsrecht. Inwieweit dieses antizipiert mittels der Betreuungsverfügung ausgeübt werden kann und wann das Gesetz der individuellen Ausgestaltung der Betreuung durch verbindliche Vorgaben Grenzen setzt, untersucht die vorliegende Arbeit. In jedem Fall stellt die Betreuungsverfügung dabei eine Möglichkeit dar, die gesetzliche Betreuung gemäß §§ 1896 ff. BGB privatautonom auszugestalten. 